\title{
Three-dimensional nonequilibrium steady state of active particles: symmetry breaking and clustering
}

\author{
Dissertation \\ zur Erlangung des mathematisch-naturwissenschaftlichen Doktorgrades \\ „Doctor rerum naturalium“ \\ der Georg-August-Universität Göttingen \\ im Promotionsprogramm ProPhys \\ der Georg-August University School of Science (GAUSS)
}

\author{
vorgelegt von \\ Rebekka Elisabeth Breier \\ aus Göttingen
}

Göttingen 2017 


\section{Betreuungsausschuss:}

Prof. Dr. Stephan Herminghaus, Dynamik komplexer Fluide, Max-Planck-Institut für Dynamik und Selbstorganisation, Göttingen

Prof. Dr. Marcus Müller, Institut für Theoretische Physik, Georg-August-Universität Göttingen

Dr. Marco G. Mazza, Dynamik komplexer Fluide, Max-Planck-Institut für Dynamik und Selbstorganisation, Göttingen

\section{Mitglieder der Prüfungskommission:}

Referent: Dr. Marco G. Mazza, Dynamik komplexer Fluide, Max-Planck-Institut für Dynamik und Selbstorganisation, Göttingen

Korreferent: Prof. Dr. Marcus Müller, Institut für Theoretische Physik, GeorgAugust-Universität Göttingen

2. Korreferent: Prof. Dr. Fabio Marchesoni, Dipartimento di Fisica, Università di Camerino, Italien

\section{Weitere Mitglieder der Prüfungskommission:}

Prof. Dr. Stephan Herminghaus, Dynamik komplexer Fluide, Max-Planck-Institut für Dynamik und Selbstorganisation, Göttingen

Prof. Dr. Annette Zippelius, Institut für Theoretische Physik, Georg-AugustUniversität Göttingen

Prof. Dr. Stefan Klumpp, Institut für Nichtlineare Dynamik, Georg-August-Universität Göttingen

Dr. Michael Wilczek, Theorie turbulenter Strömungen, Max-Planck-Institut für Dynamik und Selbstorganisation, Göttingen

Tag der mündlichen Prüfung: 2. Juni 2017 
Splay will go away

And bend will finally end

But twist will persist!

Robin L. B. Selinger, 2014 



\section{Abstract}

Motile creatures are ubiquitous in the natural world. Spanning a broad range of length scales, they all have in common the fact that they convert energy from internal or external resources into motion. In most natural situations one such individual does not exist on its own but is part of a large group like a flock of birds, a school of fish, or a bacterial suspension. Often these groups show interesting and surprising structure formation which emerges in a self-organized fashion without any external forcing. Recently, the modeling of the dynamics of such large groups has attracted a lot of interest also among physicists with the aim to understand the simple, local mechanisms which lead to a complex, global behavior.

The subject of this thesis are active particles at low Reynolds numbers in three dimensions which mimic, for example, bacteria in an aqueous environment. All particles move at a constant speed and align nematically with neighboring particles they do not distinguish between head and tail. Large groups of active particles are investigated by means of molecular dynamics simulations in the limit of overdamped dynamics.

We investigate the nonequilibrium phase diagram of these active particles in terms of density and rotational Péclet number. The latter compares the strength of the nematic alignment with the rotational diffusion. We find a phase transition from the isotropic to the nematically ordered state. Close to the transition point, traveling density waves occur which resemble solitons. In the nematic region of the phase diagram a spontaneous chiral symmetry breaking can be observed. This occurs via the formation of patterns which are characterized by a helical arrangement of the mean local orientations. We discuss their stability and study their formation. A comparison to a one-dimensional rotor model (similar to the $X Y$-model) reveals the importance of fluctuations. Very interestingly, density waves traveling along the helix emerge. They differ, however, in nature from the ones occurring at the nematicisotropic transition.

In the second part of the thesis, the active particles are immersed in a surrounding, mildly turbulent fluid $\left(\mathcal{R}_{\mathrm{A}} \approx 20\right)$ to mimic the conditions of plankton in the ocean. The fluid flow field is modeled by means of kinematic simulations to ensure reasonable computational times. However, for comparison, a number of simulations of the self-propelled particles are also performed using the result of state-of-the-art direct numerical simulations. We find a remarkably good agreement between these two methods. The particles show a turbulence-induced clustering in the form of smallscale patches in a specific region of the phase diagram. The strongest clustering occurs if the integral length scale of the vorticity of the turbulent field is equal to half of the nematic interaction range and the Kolmogorov time scale matches the time scale of nematic alignment. Finally, we discuss the implications of our results onto the famous "paradox of the plankton". 



\section{Zusammenfassung}

Viele Lebewesen bewegen sich, in dem sie ständig Energie aus einem inneren oder äußeren Energiereservoir in Bewegung umwandeln. Meist existiert ein solches Individuum nicht isoliert, sondern ist Teil einer großen Gruppe wie beispielsweise einer Vogelschar, eines Fischschwarms oder einer bakteriellen Suspension. Häufig bilden diese Gruppen ganz ohne äußere Einflüsse durch Selbstorganisation interessante und überraschende Strukturen. In jüngster Vergangenheit wurde das Interesse vieler Physiker geweckt, eben solche großen Gruppen zu modellieren um die einfachen lokalen Mechanismen zu verstehen, die genügen, komplexes globales Verhalten zu erzeugen.

Das Thema dieser Arbeit sind in allen drei Dimensionen freibewegliche, aktive Teilchen bei niedriger Reynoldszahl, die beispielsweise schwimmende Bakterien im Wasser darstellen. Jedes Teilchen bewegt sich mit konstanter Geschwindigkeit und benachbarte Teilchen haben die Tendenz sich nematisch auszurichten - sie verhalten sich wie Stäbe, die sich parallel zueinander anordnen. Wir betrachten große Gruppen solcher Teilchen, die typischerweise aus $3 \times 10^{5}$ Individuen bestehen mithilfe von überdämpften Molekulardynamik-Simulationen (Kriechfall).

Wir untersuchen das Nichtgleichgewichtsphasendiagramm dieser aktiven Teilchen in Abhängigkeit von der Dichte und der Péclet-Zahl der Rotationsbewegung, wobei letztere die Stärke der nematischen Ausrichtung mit der Fluktuationen der Orientierung vergleicht. Wir finden einen Phasenübergang von der isotropen in die nematisch geordnete Phase. An diesem Übergang treten solitonen-ähnliche, sich bewegende Dichtewellen auf. Im nematischen Bereich des Phasendiagramms beobachten wir eine spontane Brechung der chiralen Symmetrie des Systems. Diese Symmetriebrechung entsteht durch Helix-Strukturen, die sich aus den mittleren lokalen Orientierungen (im nematischen Sinne) zusammensetzen. Der Vergleich mit einem eindimensionalen, dem $X Y$-Modell sehr ähnlichen, Modell offenbart, wie wichtig Fluktuationen dabei sind. Interessanterweise enstehen dabei Dichtewellen, die sich entlang der Helixachse fortbewegen, sich jedoch von den Wellen in der Nähe des Phasenübergangs unterscheiden.

Im zweiten Teil der vorliegenden Arbeit bewegen sich die aktiven Teilchen in einer leicht turbulenten $\left(\mathcal{R}_{\lambda} \approx 20\right)$ Flüssigkeit, wie beispielsweise Plankton im Ozean. Wir modellieren die Flüssigkeit mithilfe von kinematischen Simulationen und vergleichen die Ergebnisse mit denen modernster direkter numerischer Simulationen. Die Teilchen zeigen in einem bestimmen Bereich des Phasendiagramms die Bildung von turbulenz-induzierten, kleinskaligen Haufen. Die stärkste Häufung ensteht, wenn typische Längen- und Zeitskalen des turbulenten Feldes und der aktiven Teilchen übereinstimmen. Wir diskutieren die Auswirkungen der Ergebnisse auf das berühmte "Planktonparadox". 



\section{Contents}

Abstract

Zusammenfassung iii

Contents vii

List of Symbols $\quad$ ix

List of Abbreviations $\quad$ xi

List of Figures $\quad$ xiii

List of Tables $\quad$ xvii

List of Movies $\quad$ xix

1 Introduction $\quad \mathbf{1}$

1.1 Motivation . . . . . . . . . . . . . . . . 1

1.2 Microswimmers . . . . . . . . . . . . . . . . 3

1.3 Propagating waves of self-propelled particles . . . . . . . . . 8

1.4 Motile particles in turbulent fields . . . . . . . . . . . . . . . 8

1.5 Scope of the thesis . . . . . . . . . . . . . . . . 11 
2 Theoretical background 13

2.1 Hydrodynamics of microswimmers . . . . . . . . . . . . . . . . . 13

2.1.1 Ambient fluid: Stokes equation . . . . . . . . . . . . . 14

2.1.2 Microswimmmer: Force dipole . . . . . . . . . . . . . . 16

2.2 Overdamped dynamics . . . . . . . . . . . . . . . . . . . . . . . . . . . . . . . . . . .

2.3 Turbulence. . . . . . . . . . . . . . . . . . . . . . . . 18

2.3.1 Energy spectrum . . . . . . . . . . . . . . . . . 18

2.3.2 Integral length scale . . . . . . . . . . . . . . . . 20

2.3.3 Taylor-based Reynolds number . . . . . . . . . . . . 23

3 Methods 25

3.1 Models . . . . . . . . . . . . . . . . . . 25

3.1.1 Nematically aligning self-propelled particles . . . . . . . . 25

3.1 .2 Particles with hard cores . . . . . . . . . . . . . 26

3.1.3 Particles in a turbulent field . . . . . . . . . . . . . . . . . . 28

3.1 .4 The Kraichnan flow field . . . . . . . . . . . . . . . . . . 29

3.1.5 Direct numerical simulations . . . . . . . . . . . . . . . . 38

3.2 Molecular dynamics simulations . . . . . . . . . . . . . . . . . . 39

3.2.1 Rotational noise . . . . . . . . . . . . . . . . 42

3.2.2 Monotonic logical grid . . . . . . . . . . . . . . . . . . . . . 45

3.2.3 Particles in a turbulent field . . . . . . . . . . . . . . . . . . 46

3.2.4 Boundary conditions . . . . . . . . . . . . . . . 47

3.3 Description of the system . . . . . . . . . . . . . . . . 49

3.3.1 Dimensionless measures of self-propelled particles and flow . . 50

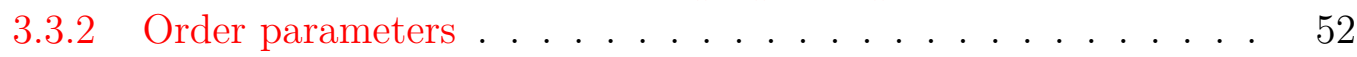

3.4 Analysis of clustering . . . . . . . . . . . . . . 56

3.4.1 Patch concentration enhancement factor . . . . . . . . 57

3.4 .2 Number fluctuations . . . . . . . . . . . . . . . 58

3.4 .3 Cluster analysis . . . . . . . . . . . . . . . 58

4 Structure formation by self-propelled point particles $\quad \mathbf{6 1}$

4.1 Phase diagram and snapshots . . . . . . . . . . . . . . . 62

4.2 Isotropic-nematic transition . . . . . . . . . . . . . . . . . 64

4.2.1 Mean-field theory . . . . . . . . . . . . . . 66

4.2 .2 Comparison to data . . . . . . . . . . . . . . . . . 69

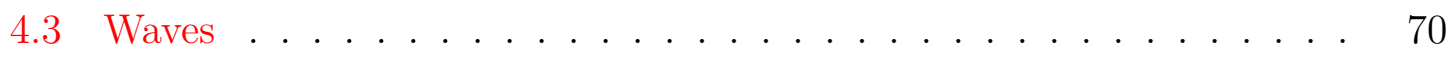

4.4 Spontaneous chiral symmetry breaking . . . . . . . . . . . . . . . . 77

4.4.1 Importance of chirality . . . . . . . . . . . . . . . 77

4.4.2 The chiral pattern . . . . . . . . . . . . . 77

4.4 .3 (Meta-)Stability of the chiral state ............. 81

4.4.4 Formation of the chiral state . . . . . . . . . . . 83 
4.4.5 Comparison to one-dimensional model . . . . . . . . . . . . 86

4.4.6 Importance of fluctuations . . . . . . . . . . . . . . . . 99 90

4.4.7 An untwisting chiral pattern . . . . . . . . . . . . 91

4.4.8 Oscillations in the polarization - A density wave? . . . . . . . 93

4.5 Influence of walls . . . . . . . . . . . . . . . . . . . . 100

4.6 Discussion . . . . . . . . . . . . . . . . . . . . . . . 104

5 Structure formation by self-propelled extended particles $\quad 109$

5.1 Nonequilibrium phase diagram and new patterns . . . . . . . . . . . . 110

5.1 .1 New phase coexistence . . . . . . . . . . . . . . 113

5.1 .2 Convection? . . . . . . . . . . . . . . . 113

5.2 Discussion . . . . . . . . . . . . . . . . . . . . . 115

6 Self-propelled particles in a turbulent field $\quad \mathbf{1 1 7}$

6.1 Nematic Order . . . . . . . . . . . . . . . . . . . . . . 118

6.1 .1 Phase diagrams . . . . . . . . . . . . . . . . . . . . 118

6.1.2 Nematic-isotropic transition . . . . . . . . . . . . . . 118

6.2 Clustering . . . . . . . . . . . . . . . . . . . . . 122

6.2.1 Point particles in a Kraichnan flow field . . . . . . . . . . . . 122

6.2.2 Extended particles in a Kraichnan flow field . . . . . . . . . . 126

6.2.3 Point particles in a DNS flow field . . . . . . . . . . . . . 131

6.2.4 Temporal evolution of cluster formation . . . . . . . . . . 131

6.2.5 Influence of integral length scale . . . . . . . . . . . . . . 134

6.2.6 Influence of Reynolds number . . . . . . . . . . . . . . . 137

6.3 Discussion . . . . . . . . . . . . . . . . . . . 137

$\begin{array}{lll}7 & \text { Conclusion and Outlook } & 143\end{array}$

7.1 Conclusion . . . . . . . . . . . . . . . . . . . . . 143

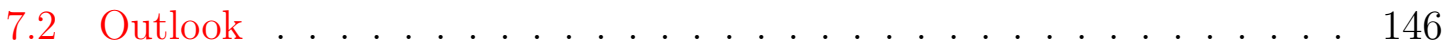

A Appendix $\mathbf{1 4 9}$

A.1 Analytical expression for $C_{\text {random }}$ in the calculation of $Q$. . . . . . . 149

A.2 Isotropic-nematic transition: Variation of time step . . . . . . . . . . 151

A.3 Isotropic-nematic transition: Finite size effects . . . . . . . . . . . . . 153

A.4 Code for three-dimensional monotonic logical grid . . . . . . . . . 153

$\begin{array}{ll}\text { Bibliography } & 157\end{array}$

$\begin{array}{ll}\text { Acknowledgements } & 171\end{array}$ 



\section{List of Symbols}

$\alpha \quad$ exponent in the analysis of number fluctuations

$\hat{d} \quad$ global nematic director

$D_{r} \quad$ rotational diffusion constant

$E(k)$ energy-spectrum function

$\hat{e}_{i} \quad$ orientation of particle $i$

$\epsilon \quad$ nematic interaction range

$\bar{\epsilon} \quad$ turbulent energy dissipation rate

$\epsilon_{F} \quad$ strength of WCA potential

$\eta_{K} \quad$ Kolmogorov length scale

$f \quad$ fraction of particles used in the calculation of $Q$

$\gamma \quad$ relaxation constant of nematic interaction

$k \quad$ turbulent kinetic energy

$L \quad$ side length of a cubic simulation box

$L_{11} \quad$ longitudinal integral length scale of velocity

$L_{11}^{\omega} \quad$ longitudinal integral length scale of vorticity

$L_{\alpha} \quad$ side length of simulation box along $\alpha$-axis

$N \quad$ number of simulated particles

$N_{2} \quad$ second moment of the cluster size distribution

$N_{F} \quad$ number of Fourier modes in turbulent field

$\vec{\omega} \quad$ turbulent vorticity

$\omega_{K} \quad$ Kolmogorov shear rate, root-mean-square vorticity of the turbulent field

$\hat{p} \quad$ direction of polarization

$\mathcal{P}$ rotational Péclet number

$P \quad$ polar order parameter

$\Phi \quad$ filling fraction in system of extended particles

$Q \quad$ patch concentration enhancement factor

$R \quad$ Reynolds number

$\vec{r}_{i} \quad$ position of particle $i$

$\mathcal{R}_{\lambda} \quad$ Reynolds number based on Taylor microscale

$S \quad$ global nematic order parameter

$S_{\chi} \quad$ chiral order parameter (cutoff $r^{*}=0.25 L$ )

$S_{\chi}^{\prime} \quad$ chiral order parameter (cutoff $r^{*}=0.4 L$ )

$\sigma \quad$ particle diameter of extended particles

$S_{\omega} \quad$ vortical Stokes number 
$S_{\omega, v} \quad$ vortical Stokes number with speed correction

$S_{\text {smec }}$ smectic order parameter

$u_{\text {rms }}$ root-mean-square velocity of the turbulent field

$\vec{u}_{\text {turb }}$ turbulent velocity

$v_{0} \quad$ self propulsion speed 


\title{
List of Abbreviations
}

\author{
DNS direct numerical simulation \\ MD molecular dynamics \\ PBC periodic boundary condition \\ SPP self-propelled particle \\ WCA Weeks-Chandler-Anderson
}





\section{List of Figures}

1.1 Examples of large groups of motile creatures . . . . . . . . . . . 2

1.2 Examples of collective behavior of motile microorganisms . . . . . . . 4

1.3 Results of the original Vicsek model (reprinted from Vicsek et al., 1995). 5

1.4 Illustration of the plankton paradox with plankton species adapted from Haeckel (1899) . . . . . . . . . . . . . . . . . . . . . . 10

3.1 Illustration of the Weeks-Chandler-Anderson (WCA) potential. . . . . 27

3.2 Illustration of the effect of hard cores modeled through the WCA force. 28

3.3 Example and probability distributions of the Kraichnan flow field. . . 36

3.4 Eulerian velocity increments of the self-propelled particles in the Kraichnan flow field. . . . . . . . . . . . . . . . . . . 37

3.5 Resulting energy spectrum of the direct numerical simulation and prescribed Kolmogorov spectrum of the Kraichnan flow field. . . . . . . . 39

3.6 Schematic structure of the molecular dynamics (MD) program. . . . . 40

3.7 Implementation of rotational stochastic noise in our model. . . . . . . 42

3.8 Illustration of the monotonic logical grid. . . . . . . . . . . . . . . 45

3.9 Run times of simulations with different numbers of particles using the MLG-algorithm or a Verlet neighborlist. . . . . . . . . . . . . 46

3.10 Sketch of different boundary conditions in the sandwich geometry. . . 48

3.11 Calculation of the chiral order parameter. . . . . . . . . . . 55

3.12 Patch enhancement factor as a function of the fraction of particles considered. . . . . . . . . . . . . . . . 5 58

3.13 Two example curves for the analysis of number fluctuations (to be published in Breier et al., 2017). . . . . . . . . . . . . . . 59

3.14 Typical examples of the cluster size distribution. . . . . . . . . . . . 60

4.1 Nonequilibrium phase diagram of self-propelled point particles with nematic interaction. . . . . . . . . . . . . . . . 62

4.2 Snapshots of the steady-state configurations of the self-propelled particles. . . . . . . . . . . . . . . . . 6 63

4.3 Critical Péclet number as a function of global number density. . . . . 65

4.4 Characterization of the wave phase. . . . . . . . . . . . . 70

4.5 Temporal evolution of the order parameters of a simulation which eventually shows the wave pattern. . . . . . . . . . . . 72 
4.6 Sketch for the wave in a system with periodic boundary conditions $(\mathrm{PBCs}) \ldots \ldots \ldots \ldots \ldots \ldots$. . . . . . . . . . . . . . . . . . . . .

4.7 Measured $\lambda_{\mathrm{m}}$ vs. calculated $\lambda_{\mathrm{c}}$ wavelength for 40 wave patterns. . . . 75

4.8 Detailed investigation of the region in the phase diagram where polarized waves occur (here: $\gamma=1$ ). . . . . . . . . . . 76

4.9 Snapshot of a chiral pattern $(\rho=1.625, \mathcal{P}=3.29$, reprinted from Breier et al., 2016). . . . . . . . . . . . . . . . . .

4.10 Chiral order parameter versus mean local nematic order parameter of metastable chiral patterns. . . . . . . . . . . . . . . . .

4.11 Investigation of different properties of the chiral pattern: Long-lived metastability and spontaneous symmetry breaking. . . . . . . . . .

4.12 Sketches of the three fundamental deformations of a nematic director field: Splay, bend, and twist (from Breier et al., 2016). . . . . . . . . 82

4.13 Temporal evolution of the chiral simulation in Fig. 4.9. . . . . . . . . 84

4.14 Emergence of the chiral pattern (adapted from Breier et al., 2016). . . 85

4.15 Results of the one-dimensional Lebwohl-Lasher model (published in Breier et al., 2016). . . . . . . . . . . . . . . .

4.16 Simulations of self-propelled particles (SPPs) with $\mathcal{P} \rightarrow \infty(\rho=1$, $\gamma=0.1 \ldots \ldots \ldots \ldots \ldots \ldots \ldots$

4.17 Evolution of a system from an isotropic state via a chiral state to a nematic state $(\rho=1.625, \mathbb{P}=6.17) \ldots \ldots \ldots 92$

4.18 Oscillations in polarization in a chiral simulation $(\rho=1, \mathbb{P}=8) \ldots .94$

4.19 Further investigation of the global polarization vector. . . . . . . . . 96

4.20 Measurement of the wavespeed of local polarization and local density (same data as in Fig. 4.18). . . . . . . . . . . . . . . .

4.21 Influence of different quantities on the oscillation period of the global polarization of chiral pattern. . . . . . . . . . . . . . . 99

4.22 Comparison of the two models for the sandwich geometry. . . . . . . 101

4.23 Nonequilibrium phase diagram (analogous to Fig. 4.1) of SPPs in a sandwich geometry using the discrete flip model. . . . . . . . . . . . 102

4.24 Two-directional pattern in the system with sandwich geometry $(\mathcal{P}=$ $4.1, \rho=1.75) \ldots \ldots \ldots \ldots \ldots$

4.25 Temporal evolution of the global order parameters in the simulation which shows two main directions of local nematic directors (sandwich geometry, see Fig. 4.24). . . . . . . . . . . . . . . . . . .

5.1 Nonequilibrium phase diagram of extended, self-propelled, nematically aligning particles. . . . . . . . . . . . . . . .

5.2 Steady-state snapshot of a phase coexistence pattern occurring in the system of extended particles $(\mathcal{P}=2.22, \rho=4)$ which does not occur for point-like particles. . . . . . . . . . . . . . . . . . 
5.3 Analysis of the new phase coexistence state (see snapshot in Fig. 5.2). 114

5.4 Steady-state configuration of a system which exhibits a convection pattern $(\rho=5, \mathbb{P}=2.28) \ldots \ldots \ldots \ldots 114$

6.1 Nonequilibrium phase diagram of self-propelled particles in a turbulent flow (figures to be published in Breier et al., 2017, supplementary information). . . . . . . . . . . . . . . . .

6.2 Nematic order parameter as a function of vortical Stokes number with speed correction (figure to be published in Breier et al., 2017, supplementary information). . . . . . . . . . . . . . .

6.3 Influence of the global number density onto the nematic-isotropic transition of point particles in the Kraichnan flow field. . . . . . . . . . .

6.4 Clustering of point particles in a Kraichnan flow field ((a) and (b) to be published in Breier et al., 2017, (b) in supplementary information). 123

6.5 Typical snapshots of point particles in the Kraichnan flow field. . . . 125

6.6 Clustering of hard-core particles in the Kraichnan flow field ((a) and (b) to be published in Breier et al., 2017, (b) in supplementary information).127

6.7 Typical snapshots of hard-core particles in Kraichnan flow field analogously to Fig. 6.5. . . . . . . . . . . . . . . . . . . . . . . . . . . 128

6.8 Extended particles in a Kraichnan flow field at low filling fraction $(N=$ $\left.8000, \phi=0.24 \%, \boldsymbol{P}=1.38 \times 10^{5}, v_{0} / u_{\mathrm{rms}}=8.5\right) \ldots \ldots \ldots 130$

6.9 Clustering of point particles in the direct numerical simulation (DNS) flow field as a function of vortical Stokes number and Péclet number ((a) to be published in Breier et al., 2017). . . . . . . . . . . . . . . . 132

6.10 Formation of a clustered state (to be published in Breier et al., 2017).

6.11 Influence of the integral length scale of the vorticity on the isotropicnematic transition and on the clustering (point particles in Kraichnan flow field). . . . . . . . . . . . . . . . . . . .

6.12 Maximum patch concentration enhancement factor as a function of integral length scale (to be published in Breier et al., 2017). . . . . .

6.13 Influence of Taylor-based Reynolds number on simulations with point particles in the Kraichnan flow field (to be published in Breier et al., 2017, supplementary information). . . . . . . . . . . . . .

6.14 Illustration of the consequence of turbulence induced clustering on different phytoplankton species (cf. Fig. 1.4). . . . . . . . . . . . . . 141

A.1 Analysis of $C_{\text {random }}(f)$ for point-like and extended particles. . . . . . . 150

A.2 Influence of the time step $\Delta t$ on the isotropic-nematic transition. . . . 151

A.3 Analysis of finite-size effects on the isotropic-nematic transition ( $\rho=$ $0.5, \gamma=1)$ for different numbers of particles as indicated. . . . . . 152 



\section{List of Tables}

3.1 Input parameters for the simulations. . . . . . . . . . . . . . . . 41

4.1 Input parameters for point-like self-propelled particles. . . . . . . . 61

4.2 Results of 300 independent simulations per value of $P$ at $\rho=1$. (data published in Breier et al., 2016, we used a different definition of Péclet number there $\ldots \ldots \ldots \ldots$

5.1 Input parameters for extended self-propelled particles. . . . . . . . . 110

6.1 Input parameters for the simulations of particles in a turbulent field. 118

6.2 Comparison between flow fields from DNSs and kinematic simulations. 139 



\section{List of Movies}

\begin{tabular}{ll}
\hline Name of file & Contents \\
\hline wave.avi & Wave pattern of self-propelled, point-like parti- \\
& cles, see Section 4.3. A fraction of the particles \\
& is shown with their orientation vectors as little \\
& arrows. The local nematic order parameter is \\
& given in color. \\
& Phase coexistence state of self-propelled, ex- \\
& tended particles, see Section 5.1.1. The right \\
hardcores_phase_coexistence.avi & panel shows a fraction of the particles with ori- \\
& entations as little arrows and local nematic or- \\
& der parameter in color. The left panels show \\
& the temporal evolution of four global order \\
& parameter (from top to bottom): nematic $S$, \\
& smectic $S_{\text {smec }}$, polar $P$ (called ferromagnetic), \\
& and chiral $S_{\chi}$. \\
\hline
\end{tabular}





\section{Introduction}

\subsection{Motivation}

Motile creatures form a large and very important part of nature. Especially large groups of animals which move together are fascinating due to the beauty of their coherent motion. One such example is a flock of starlings in the evening sky (Fig. 1.1a) constantly changing the shape of the flock while not leaving behind even a single individual. And, most importantly, this whole spectacle is presented without any external influence but a result of self-organization of the individuals. The study of the collective behavior of such groups of animals has attracted a lot of interest of researchers because these systems are intrinsically out of equilibrium, that is, they constantly convert energy into motion. Systems under investigation include for instance herds of sheep (Fig. 1.1b, Garcimartín et al., 2015; Ginelli et al., 2015), schools of fish (Fig. 1.1c, Katz et al., 2011), and, of course, flocks of birds (Ballerini et al., 2008; Cavagna et al., 2010; Pearce et al., 2014; Attanasi et al., 2014). Apart from the investigation of wild-life animals, also the collective behavior of human crowds is studied. Clearly, it is important to understand how crowd disasters such as crushes and stampedes can be avoided, and also how large groups of pedestrians can safely arrive at their destinations (Moussaïd et al., 2011).

All of the mentioned creatures up to this point belong to the group of vertebrates and clearly their importance in nature is out of question. However, the behavior of vertebrates in nature can be rather complex because any decision they make is based on a number of influences like the signals coming from their senses, learned social behavior, and also their personal history. The decision itself then requires some kind 


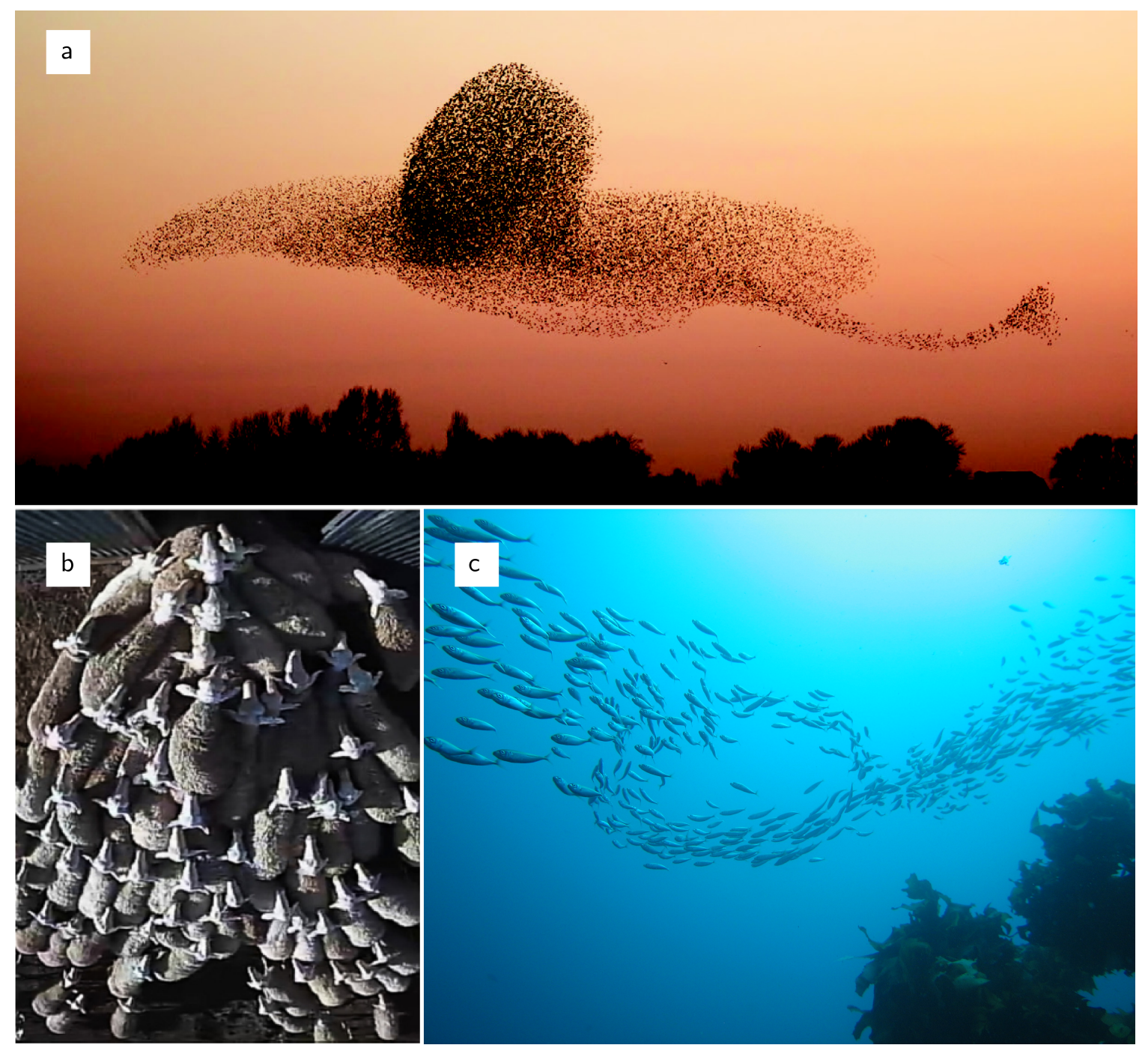

Figure 1.1: Examples of large groups of motile creatures: (a) a flock of starlings $^{1},(\mathrm{~b})$ a clogging herd of sheep ${ }^{2}$, (c) a school of fish ${ }^{3}$.

\footnotetext{
${ }^{1}$ Reprinted by permission from Macmillan Publishers Ltd: Nature (Popkin, Nature 529(7584):1618, 2016), copyright 2016. doi: https://doi.org/10.1038/529016a.

${ }^{2}$ Reprinted figure with permission from Garcimartín et al., Physical Review E 91(2):022808, 2015. Copyright 2015 by the American Physical Society. doi: https://doi.org/10.1103/PhysRevE. 91.022808.

${ }^{3}$ Photograph by Joanna Penn, source: https://www.flickr.com/photos/38314728@N08/ 3997721496, license: CC-BY-2.0 https://creativecommons.org/licenses/by/2.0/.
} 
of intelligent behavior which can be seen as the processing of all stimuli and sensory information. This means that any study of the single-entity or even collective behavior has to take into account different kinds of influences which makes it complicated to deduce true causalities. Corresponding modeling approaches can either neglect some of the influences a priori to keep it simple at the risk of over-simplification, or include as many detailed mechanisms as possible which will lead to complicated models where it might be hard to understand the direct effect of a single influence. To avoid all these problems and risks, one can also turn to organisms which are simpler in itself so that the number of influencing factors is limited. The vast world of microorganisms includes many different kinds from bacteria and archaea to fungi and microalgae. Even though not directly visible to the eye, they are of extreme importance in nature: They help us in processes such as digestion, decomposition, food production, energy production in biogas reactors, production of chemicals or enzymes, et cetera. Many microbes are motile and often perform different kinds of taxis - directed motion along a gradient of stimulus. This can be for instance aerotaxis along an oxygen gradient (Taylor et al., 1999), chemotaxis along a chemical gradient (Berg, 1975), phototaxis due to light or gravitaxis due to gravity. The latter two are especially important for marine microorganisms like dinoflagellates (Eggersdorfer and Häder, 1991). Large groups of microorganisms can show fascinating collective behavior: The gliding bacterium Myxococcus xanthus exhibits density variations in form of ripples (Figs. 1.2a and b, Berleman et al., 2008; Zhang et al., 2012a). Collective behavior can help a bacterial colony to survive by avoiding age defects (Vedel et al., 2016). Motile bacteria which perform aerotaxis can form dense bands depending on the preferred oxygen concentration (Mazzag et al., 2003). Bacteria at high density in quasi two-dimensional as well as in three-dimensional geometries can show patterns which resemble turbulence - not only from visual inspection but also in terms of energy spectra and structure functions (Fig. 1.2c, Wensink et al., 2012). And if they are confined, a single vortex is stabilized (Fig. 1.2d, Wioland et al., 2013; Lushi et al., 2014). The latter is an example of emerging chirality in the system, the importance of which we will discuss in Chapter 4.

\subsection{Microswimmers}

Microorganisms or artificial particles that are motile and interact with a surrounding fluid are referred to as microswimmers. Those include, for example, bacteria with flagella, whose spinning motion creates the self-propulsion of the organism. Besides these natural microswimmers, also artificial microswimmers can be manufactured and studied like remotely powered miniature diodes (Chang et al., 2007); micro-emulsion droplets (Thutupalli et al., 2011); self-diffusiophoretic, asymmetric swimmers (Hagen et al., 2014); and ferromagnetic particles in an oscillating magnetic field (Grosjean 

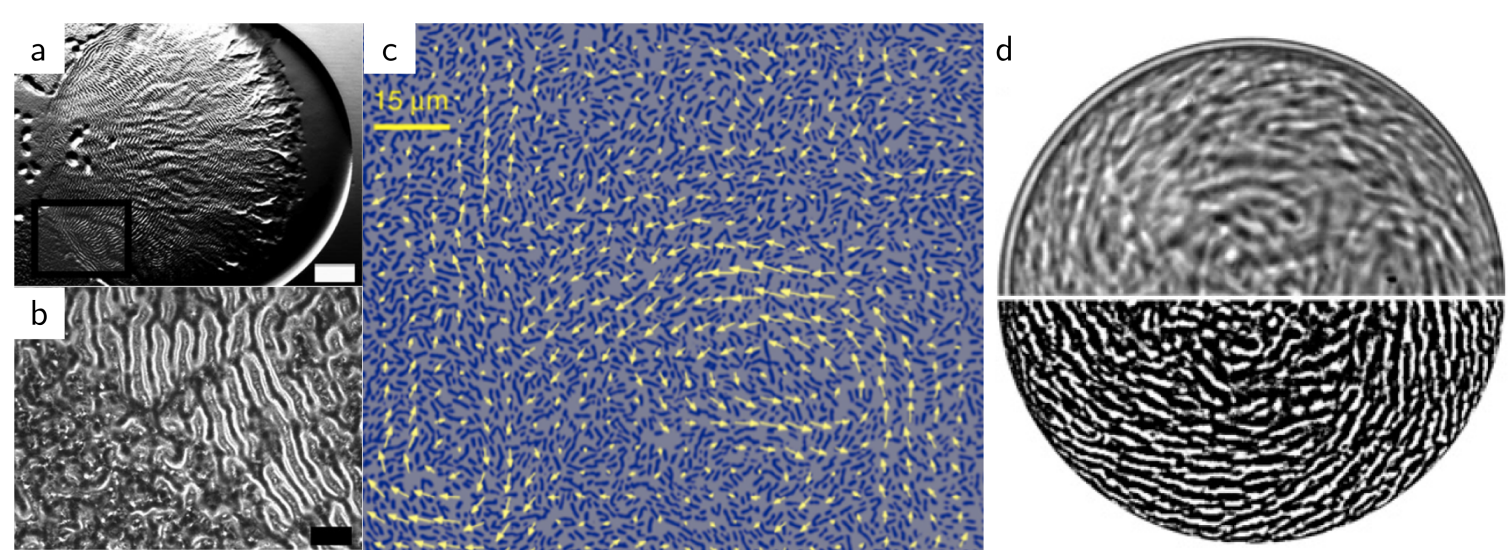

Figure 1.2: Examples of collective behavior of motile microorganisms: (a)\&(b) formation of ripples by Myxococcus xanthus during predation (reprinted from Berleman et al., 2008, Copyright 2008 National Academy of Sciences), (c) bacterial turbulence observed in a suspension of Bacillus subtilis (reprinted from Aranson, 2013, image: Andrey Sokolov, Argonne National Laboratory), (d) formation of a single vortex in a confined suspension of Bacillus subtilis (reprinted from Lushi et al., 2014, Copyright 2014 National Academy of Sciences).

et al., 2016) ${ }^{4}$. One important feature of any microswimmer is that it swims at low Reynolds numbers, typically $\mathcal{R}=10^{-5}-10^{-4}$ (Purcell, 1977). The Reynolds number $\mathcal{R}$ measures quite generally the ratio between inertial and viscous forces. A low Reynolds number hence means that inertia is not important while viscous friction dominates the system. A widely-used analogy to this regime is that a human swimming in a swimming pool full of honey would feel this condition. It is important to note that at low Reynolds number a time-reversible motion would not produce net displacement (this is called the "scallop theorem"). Based on the swimming pattern, we can distinguish two main types of microswimmers: pushers and pullers (Lauga and Powers, 2009). Pullers, like the microalga Chlamydomonas, possess two flagella at the front of the cell body which perform a breaststroke-like motion. They are called pullers because they pull the fluid towards them. On the other hand pushers like the bacterium Escherichia coli propel themselves with a rotating bundle of flagella on their cell body. Thus they push the fluid away from their body. Some pushers (like spermatozoa) possess only a single flagellum at the back which oscillates but acts similarly on the surrounding fluid like a bundle of flagella. One typical motion pattern of microswimmers is the run-and-tumble motion. E. coli is one of the model species which follow this motion pattern. When the flagella are rotating as a bundle, the organism moves forward. However, the bundle can spontaneously disintegrate

${ }^{4}$ These ferromagnetic particles are a realization of the "simplest swimmer at low Reynolds number" proposed by Najafi and Golestanian (2004). 

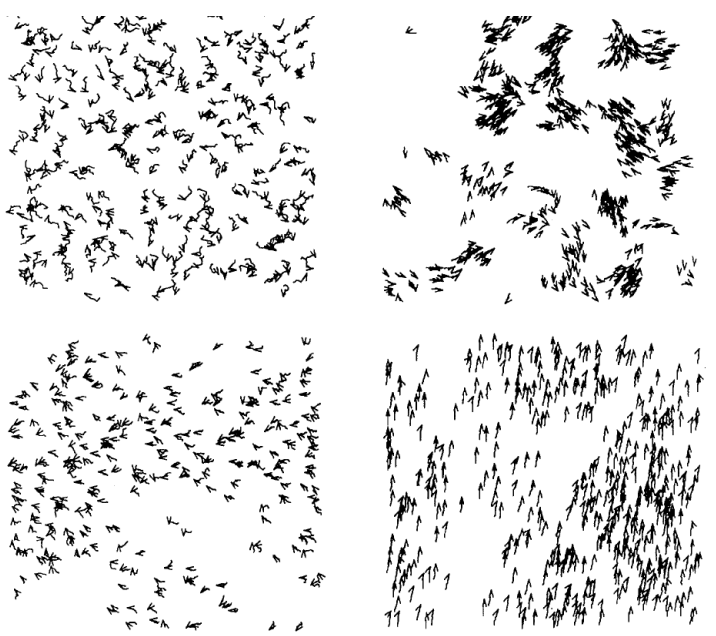

(a) Steady-state configurations at different densities and noise levels. The velocity of each particle is indicated by a little arrow together with a short curve for the trajectory of the last 20 time steps.

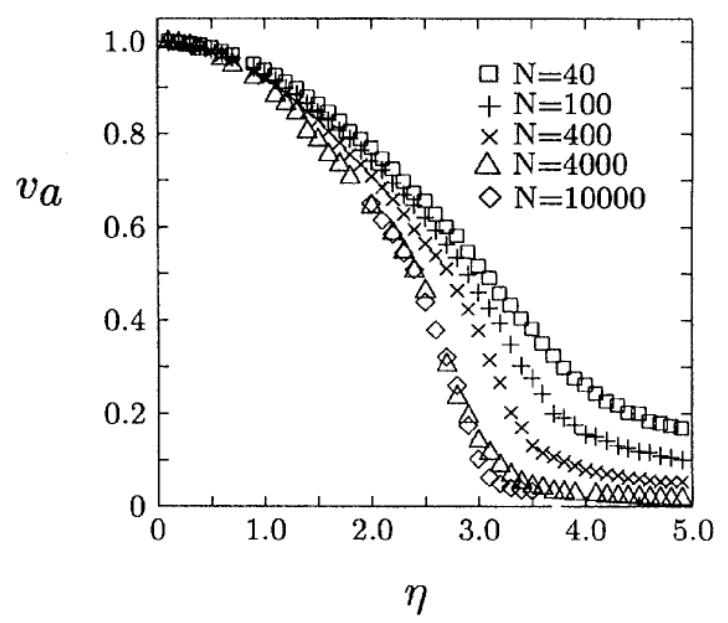

(b) Phase transition observed in the original Vicsek model: mean velocity $v_{a}$ as a function of noise $\eta$ for different numbers $N$ of particles.

Figure 1.3: Results of the original Vicsek model. Reprinted figure with permission from Vicsek et al., Physical Review Letters 75(6):1226, 1995. Copyright 1995 by the American Physical Society. doi: https://doi.org/10.1103/PhysRevLett.75. 1226.

with all flagella moving independently; the microorganism tumbles until the bundle forms again (Berg, 2004, p. 39). Other microorganisms, like Vibrio alginolyticus, only possess a single flagellum and perform a run-reverse-flick motion pattern (Xie et al., 2011; Stocker, 2011). Such a microswimmer first moves in a straight line ("run"), then turns around by an angle close to $\pi$ ("reverse"), moves straight again, and finally turns be roughly $\pi / 2$ ("flick").

The subject of this thesis are large groups of self-propelled particles and their collective behavior. One approach to investigate this subject is to model them computationally. The idea to model the behavior of a flock of birds by simple interactions between individuals which lead to a complex collective behavior was first introduced in the field of computer graphics (Reynolds, 1987). A few years later, Vicsek et al. (1995) published their seminal work on a very simple model of point-like particles which move at a constant speed in two dimensions. At every time step, each particle reorients into the mean direction of its neighbors and experiences a random rotational noise. The beauty of this work lies in the few ingredients which suffice to trigger a phase transition from an ordered state to a disordered phase (see Fig. 1.3b). This ordered phase is characterized by a collective motion of the center of mass of the par- 
ticles such that the rotational symmetry of the system is broken (see Fig. 1.3a). In the following decades a large zoo of similar models has been proposed by a number of researchers to study different aspects of the collective behavior of active particles (review by Vicsek and Zafeiris, 2012). One group of models excludes any alignment rules whatsoever: the only interaction between two particles is based on their relative distance; it can typically be described by a pair-wise potential. Moreover, each particle itself aims to keep a prescribed mean speed. A phase transition (Wysocki et al., 2014) as well as collective behavior (Grossman et al., 2008) can be observed. Even a chiral symmetry breaking in terms of a rotational mode was found for a three-dimensional model of particles interacting via a potential (Erdmann and Ebeling, 2003; Erdmann et al., 2005; Strefler et al., 2008; Romanczuk et al., 2012; Jiang et al., 2016).

The second group of models, on which we will focus here, are models of active particles with some alignment mechanism. They all somehow "descend" from the previously described Vicsek model. Two main different alignment rules can be identified: (i) the particles exhibit polar alignment as in the original Vicsek model where a given particle calculates the mean velocity of its neighbors hence distinguishing between head and tail of neighboring particles. (ii) The particles' alignment rule has nematic symmetry, that is, a given particle does not distinguish head and tail of its neighbors. This interaction rule physically corresponds to rod-like particles that tend to align. However, the motion of each particle is still directed, i.e., it possesses a head and a tail.

A large amount of research has been performed on polarly interacting particles: The Vicsek model has been extended to three dimensions (Czirók et al., 1999) where again a phase transition between an ordered and a disordered state is found. The order of the phase transition has been investigated in both two and three dimensions (Chaté et al., 2008b). Possible extensions of the simple two dimensional model include the addition of cohesive effects between particles (Grégoire and Chaté, 2004), the interaction with non-active particles as obstacles (Chepizhko et al., 2013), an additional deflection of particles just after alignment (Meschede and Hallatschek, 2013), and the introduction of bounded confidence to the swimmers (Romensky et al., 2014). Chaté et al. (2008a) briefly review the work on models similar to the Vicsek model (up to that point) and propose three different pathways to extend the original Vicsek model: change the polarity of the interaction as well as of the particles themselves, add cohesion, and model the interaction with an ambient fluid. A number of authors have investigated a variation of the Vicsek model in two dimensions with nematic interactions (Chaté et al., 2006; Ginelli et al., 2010). Again, this model can be extended for example by giving the particles a memory (Nagai et al., 2015). All these models have in common that they are time-discrete (just like the original Vicsek model) with an instantaneous alignment towards a mean orientation of the neighboring particles. A time-continuous approach has been proposed (Peruani et al., 2008) which incorporates a potential to describe the alignment mechanism. This introduces a new time 
scale into the system, the time scale of (polar or nematic) alignment.

The interactions of these models are all based on metric rules; a particle interacts with all of its neighbors within a certain distance. However, a topological rule is also justified since a given self-propelled particle in nature might only interact with its nearest neighbors. The importance of the topological distance rather than the metric distance for interacting birds in a flock can be deduced from field studies (Ballerini et al., 2008). A Vicsek-type model with topological interactions also leads to qualitatively different results than the one with metric interactions (Ginelli and Chaté, 2010). A similar ansatz is followed by Barberis and Peruani (2016) who introduce a cognitive flocking model where interaction is based on visual perception only (with a varying vision cone).

Another route to make the Vicsek model more realistic is the introduction of different alignment mechanisms (polar and nematic) in one and the same system. For example, a binary mixture of self-propelled particles is of interest where particles of the same species align polarly, while particles of different species either align polarly, nematically, or do not align but prefer a perpendicular arrangement (Menzel, 2012). A competition between both nematic and polar alignment can be realized either by giving a finite probability to both interaction mechanisms (Ngo et al., 2012) or by using one mechanism for short range interactions and one for long range interactions (Großmann et al., 2014).

While all of the reported simulations with alignment mechanism use point-like particles, extended particles are of course also of interest (Weitz et al., 2015). Another interesting model includes soft, deformable particles (Menzel and Ohta, 2012).

Apart from the individual-based models, a hydrodynamic and mean-field approach to self-propelled particles is useful to study e.g. the nature of the phase transition. Toner and $\mathrm{Tu}(1995,1998)$ were the first to develop a hydrodynamic model to describe this nonequilibrium system and its important differences to the corresponding equilibrium model. Instabilities of the ordered system to fluctuations were predicted by Aditi Simha and Ramaswamy (2002), whose results were later generalized by Saintillan and Shelley (2008). A Boltzmann equation approach was introduced by Bertin et al. $(2006,2009)$ and refined as a Boltzmann-Ginzburg-Landau approach by Peshkov et al. (2014). The results of Baskaran and Marchetti (2008b,a, 2012) suggest possible steady states of a two-dimensional system of nematically interacting particles based on a hydrodynamic model which is derived from a physical minimal model. Finally, the kinetic approach of Ihle $(2011,2013)$ relates the order of the phase transition to the occurrence of instabilities as waves. Reviews of the hydrodynamics of active particles were done by Toner et al. (2005); Lauga and Powers (2009); Marchetti et al. (2013). A rather simple mean-field approach was proposed by Peruani et al. (2008) which predicts the locus of the order-disorder transition line for two dimensional systems with polar or nematic alignment. 


\subsection{Propagating waves of self-propelled particles}

The emergence of collective behavior is an important feature of many active systems in nature. Driven actin filaments show (among others) the emergence of large, interconnected, long-lived bands (Schaller et al., 2010, 2011). These bands are interpreted as density waves emerging in the system due to specific alignment mechanisms. A second model system to study collective behavior is the gliding bacterial species Myxococcus xanthus (Zhang et al., 2012b). This social bacterium forms a rippling structure during predation (see Figs. 1.2a and b; Berleman et al., 2008). Corresponding simple models reveal the importance of few ingredients for the ripple formation: reversals, a refractory period between reversals, and local alignment (Wu et al., 2009; Zhang et al., 2012a). The phase transition to collective motion in a model mimicking gliding bacteria can be shown to occur via clustering (Peruani et al., 2012).

Apart from the biological motivation due to the occurrence of waves in "real-world systems", also the self-propelled particles in minimal models in the spirit of the Vicsek model can form dense, propagating bands. Such density wave occur in the original Vicsek model for large velocities with a concurrent alteration of the nature of the order-disorder phase transition (Nagy et al., 2007). Self-propelled particles in variants of the Vicsek model also form waves when they have bounded confidence (Romensky et al., 2014) or posses memory (Nagai et al., 2015). Even models of soft, extended, self-propelled particles without an explicit alignment lead to traveling bands close to the phase transition (Ohta and Yamanaka, 2014; Yamanaka and Ohta, 2014).

In addition to agent-based models, continuum theories also suggest the formation of bands. Density-segregated, banded solutions are found using a Boltzmann approach (Bertin et al., 2006, 2009; Peshkov et al., 2012). Kinetic theory suggests a similar density profile as agent-based simulations (Ihle, 2013). The formation of the traveling bands is typically found close the order-disorder transition, for example, as a banding instability in hydrodynamic theory of polarly aligning SPPs (Baskaran and Marchetti, 2012). Moreover, the banding instability alters the nature of the phase transition (shown by kinetic theory, Ihle, 2013) or the (tri-) critical point (in the Vicsek model with bounded confidence, Romensky et al., 2014).

\subsection{Motile particles in turbulent fields}

Microorganisms live in many different habitats, ranging from soil and rocks, water and air, to mucus and skin of possible host species. Microorganisms existing in an aqueous environment have attracted considerable interest in the literature; recent examples are bacteria (Baskaran and Marchetti, 2009; Zhang et al., 2012a; Peruani et al., 2012; Lushi et al., 2014), zooplankton (Omori and Hamner, 1982; Seuront et al., 2004), phytoplankton (Durham et al., 2013) or sperm cells (Riedel et al., 
2005). The natural environment of these microbes is often found to be turbulent, especially large water bodies like oceans and lakes. We will hence focus on plankton in the following as a model group of microorganisms in a turbulent environment. The term plankton is a collective term for a large variety of species which live in large water bodies and in general passively drift in the currents (Lalli and Parsons, 1993). However, many plankton species are motile (Visser and Kiørboe, 2006) and often perform gyrotaxis. This is a directed motion resulting from the reorientation of the swimmer due to gravitational and viscous torques. Gyrotactic swimmers are often bottom-heavy which leads to a vertical adjustment of their swimming direction and the swimmers become focused in a down-welling, laminar flow (Kessler, 1985). In a turbulent environment, gyrotactic microorganisms show the formation of smallscale patches in experiments and simulations which also typically occur in the downwelling regions of the flow field (Durham et al., 2013). Simulations suggest that the shape of the microorganisms (either spherical or elongated) is crucial to determine the degree of clustering (Zhan et al., 2014). A statistical model can be used to support these findings and quantify the effect of the particle shape on the cluster formation (Gustavsson et al., 2016). Vertically migrating phytoplankton forms patches also in its natural habitats like in the sea (Ross and Sharples, 2007) or in lakes (Alexander and Imberger, 2009). A combined study of the turbulent flow field as well as the occurrence of motile phytoplankton (Ceratium) reveals that one of its habitats is the pycnocline (Maar et al., 2003) for example in the Skagerrak (North Sea). This is the layer of the steepest density gradient which occurs in many large water bodies. The measurement of the corresponding turbulent field indicates a very mild turbulent field (Taylor-based Reynolds number $\mathcal{R}_{\lambda} \approx 20$ ).

Plankton species often occur in small-scale patches (Davis et al., 1992). Such a clustering leads to hotspots of fish (Tiselius, 1992) and is hence important to fishing. Moreover, it increases the species diversity (Richerson et al., 1970) and influences the predator-prey dynamics as well as the rates of fish recruitment (Pitchford and Brindley, 2001). Furthermore, the patchiness has an impact on the population stability (Steele, 1974).

One big unresolved question in marine biology and ecology is the so-called "paradox of the plankton" (Fig. 1.4). This term has been introduced by Hutchinson (1961) who asked how it is possible that we find a large variety of plankton (especially phytoplankton) in large lakes and the oceans which are all competing for the same resources - light and nutrients. These nutrients are limited and hence "competitive exclusion" tells us that only one single species should survive. However, we find instead a large variety of different species in the oceans and lakes. A lot of research on this topic has been performed since Hutchinson introduced the problem but no unifying theory has been found to date. We will review the most important possible solutions in the following; more details can be found in the review of Roy and Chattopadhyay (2007).

Two main mechanisms have been proposed to resolve the paradox of the plankton: 


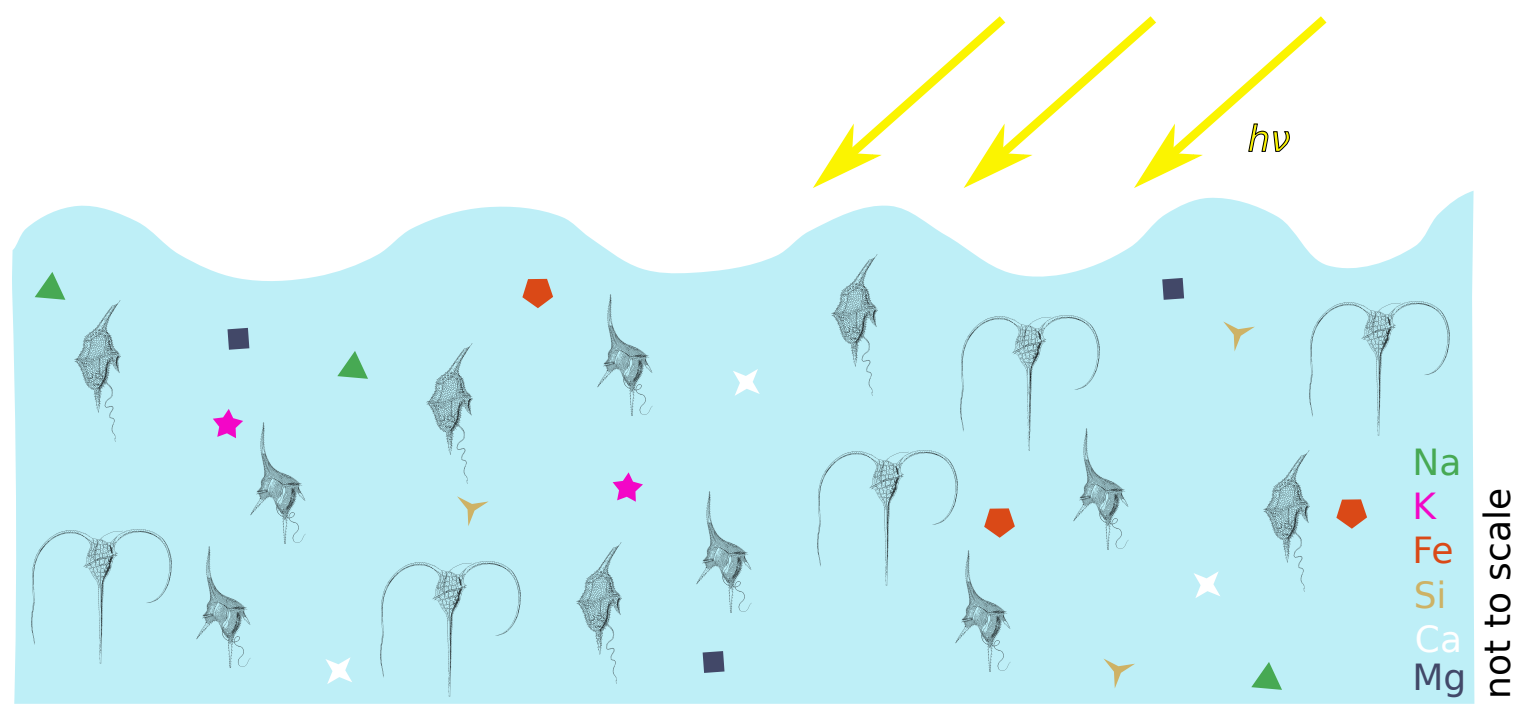

Figure 1.4: Illustration of the plankton paradox: A large variety of species competes for a limited number of nutrients and light. The phytoplankton species Ceratium tripos, Ceratium cornutum, and Ceratium hirundinella are adapted from Haeckel (1899, online: http://biolib.mpipz.mpg.de/haeckel/kunstformen/natur.html, copyright 1999 Kurt Stueber und Max-Planck-Institut für Züchtungsforschung).

Either the system never reaches equilibrium, or there are additional limiting factors which have to be accounted for. The system could be brought out of equilibrium by external forcing dynamics: This includes purely temporal effects like a separation of time scales of reproduction and environmental times scales (Hutchinson, 1961). Different models showed that coexistence of competing species can be achieved by a nutrient in pulses (Ebenhöh, 1988), temperature fluctuations (Descamps-Julien and Gonzalez, 2005), or seasonal forcing which leads to chaotic behavior (Scheffer et al., 2003). Proposed spatio-temporal effects leading to nonequilibrium dynamics are for example the heterogeneity of the plankton habitat (Richerson et al., 1970), coherent vortices (Bracco et al., 2000), and incomplete mixing (Levin, 1974; Atkinson and Shorrocks, 1981). Another route how the plankton system might be driven out of equilibrium is the emergence of self-organized dynamics in the system. The analysis of plankton models suggest for instance the existence of limit cycles (Armstrong and McGehee, 1980) or the emergence of chaos (Gragnani et al., 1999). The latter has even been shown experimentally in a real plankton community (Benincà et al., 2008). Apart from the assumption that the system of competing plankton species is out of equilibrium, additional limiting factors might play a role and lead to the coexistence of a large number of species. Conceivable mechanisms include different biological parameters (Huisman et al., 2001) or metabolic strategies (Posfai et al., 2017), as well as predator-prey interactions. Moreover, some plankton species are 
able to produce toxin which leads to a self-limitation (Roy et al., 2007).

\subsection{Scope of the thesis}

This thesis aims to understand the complex steady-state phase space of nematically aligning, self-propelled particles in three dimensions. As discussed before, selfpropelled particles are ubiquitous in nature and a large number of them are not confined to surfaces but can move freely in three dimensions. Moreover, the hydrodynamic interactions are of nematic symmetry to first order (Baskaran and Marchetti, 2009) so that nematic alignment rules are justified. We do not explicitly take into account hydrodynamic interactions between the particles which is why we rather use the term "self-propelled" or "active" particle than "microswimmer". We know that propagating density waves occur in two-dimensional systems and want to study their occurrence and properties in three dimensions. Moreover, understanding the spontaneous breaking of chiral symmetry in an achiral system is crucial to explain the emergence of life. This thesis adds one small component to the discussion by investigating the role of fluctuations in the formation process. A more realistic model should deal with extended particles instead of point-like ones. Very little has been done in the past in the field of self-propelled, extended particles with local alignment. So we ask the questions how steric interactions between particles influence the phase diagram and especially the steady-state configurations.

In the second part of the thesis, we deal with self-propelled -both point-like and extended-particles immersed in a turbulent flow field. We want to understand how the interplay between alignment and turbulent flow field influences the formation of small-scale patches of the particles such as the plankton found in the ocean. This is an extension of the work performed by many researchers on gyrotactic, self-propelled particles since our nematically aligning particles do not have a preferred direction like gyrotactic particles do. It is hence a question of the self-organization of the particles as a group rather than their interaction with an outer (gravitational) field. Moreover, we address the question how a turbulent flow field can broaden the current understanding of the paradox of the plankton, at least for motile plankton.

The thesis is organized as follows: Chapter 2 gives a short overview over the theory of overdamped dynamics including the justification for nematic alignment, as well as basic principles of turbulent flows. The model of self-propelled, aligning particles is introduced in Chapter 3 for both point-like as well as extended particles. The specific method for the turbulent flow field is explained in detail as well as the numerical implementation of the models. Order and control parameters of the system are presented and we describe different methods to measure clustering in a system of particles. The results of self-propelled, aligning, point-like particles are presented in Chapter 4 in terms of the nonequilibrium phase diagram. Large weight is given 
to the density waves and to the chiral symmetry breaking. The main findings of the latter were published in Breier et al. (2016). Chapter 5 deals with extended, selfpropelled, aligning particles and the corresponding phase diagram. In Chapter 6 the self-propelled particles are immersed in a turbulent field; the resulting nonequilibrium phase diagram and especially the turbulence-induced clustering is investigated. Part of the results presented in Chapter 6 were developed in the bachelor's thesis of D. Waas (2015). The main findings will be published in Breier et al. (2017). 


\section{Theoretical background}

This Chapter gives a brief overview of the theoretical background of our model. We discuss the hydrodynamics of microswimmers and the symmetry of their interaction in Section 2.1 to motivate our model of nematically aligning, self-propelled particles. In Section 2.2, we examine the importance of the Reynolds number in this context and then introduce the related concept of overdamped dynamics along with the Langevin equation. We want to study self-propelled particles in a turbulent field and therefore present some basic concepts from the description and analysis of turbulent flow fields in Section 2.3.

\subsection{Hydrodynamics of microswimmers}

This thesis deals with aligning, self-propelled particles which mimic microswimmers, i.e. self-propelled particles which move inside a fluid. This means that hydrodynamic interactions have to be taken into account. In the following, we will sketch how the hydrodynamics of a simple model of microswimmers can be derived as proposed by Baskaran and Marchetti (2009). 


\subsubsection{Ambient fluid: Stokes equation}

The surrounding fluid of the microswimmers is in general described by the famous incompressible Navier-Stokes equations (Kundu and Cohen, 2008, p. 426):

$$
\begin{aligned}
\rho \frac{\partial \vec{u}}{\partial t}+\rho(\vec{u} \cdot \nabla) \vec{u} & =\vec{f}-\nabla p+\mu \nabla^{2} \vec{u} \quad \text { and } \\
\nabla \cdot \vec{u} & =0
\end{aligned}
$$

with the density $\rho$, the flow velocity $\vec{u}$, the pressure $p$, and the dynamic viscosity $\mu$. The term $\vec{f}$ is the body force density which accounts for any external forces on the fluid. We can non-dimensionalize Eq. (2.1a) by expressing the velocity as well as the temporal and positional derivatives in terms of characteristic values

$$
\begin{aligned}
\vec{u} & =U \vec{u}^{\prime}, \\
\frac{\partial}{\partial t} & =\frac{1}{T} \frac{\partial}{\partial t^{\prime}}, \\
\nabla & =\frac{1}{L} \nabla^{\prime},
\end{aligned}
$$

where $U, L, T$ are the typical speed, length, and time of the flow, respectively, and all primed quantities are dimensionless. The typical speed can be calculated from the typical time and length $U=L / T$. Inserting Eqns. (2.2) into Eq. (2.1a) yields

$$
\begin{aligned}
\rho \frac{U}{T} \frac{\partial \vec{u}^{\prime}}{\partial t^{\prime}}+\rho \frac{U^{2}}{L}\left(\vec{u}^{\prime} \cdot \nabla^{\prime}\right) \vec{u}^{\prime} & =\vec{f}-\frac{1}{L} \nabla^{\prime} p+\frac{\mu U}{L^{2}} \nabla^{\prime 2} \vec{u}^{\prime} \\
\Rightarrow \frac{\rho U L}{\mu}\left(\frac{\partial \vec{u}^{\prime}}{\partial t^{\prime}}+\left(\vec{u}^{\prime} \cdot \nabla^{\prime}\right) \vec{u}^{\prime}\right) & =\frac{L^{2}}{\mu U} \vec{f}-\frac{L}{\mu U} \nabla^{\prime} p+\nabla^{\prime 2} \vec{u}^{\prime} \\
\Rightarrow \frac{\rho U L}{\mu}\left(\frac{\partial \vec{u}^{\prime}}{\partial t^{\prime}}+\left(\vec{u}^{\prime} \cdot \nabla^{\prime}\right) \vec{u}^{\prime}\right) & =\vec{f}^{\prime}-\nabla^{\prime} p^{\prime}+\nabla^{\prime 2} \vec{u}^{\prime} .
\end{aligned}
$$

In the last step the force density and the pressure have been replaced by their dimensionless equivalents

$$
\begin{aligned}
\vec{f}^{\prime} & =\frac{L^{2}}{\mu U} \vec{f} \text { and } \\
p^{\prime} & =\frac{L}{\mu U} p .
\end{aligned}
$$

The left-hand side of Eq. (2.3) is proportional to the dimensionless number $\rho U L / \mu$ which is the Reynolds number. It can be defined (see Kundu and Cohen, 2008, p. 292) as the ratio of inertial forces over viscous forces. The order of magnitude of 
the corresponding terms $\rho(\vec{u} \cdot \nabla) \vec{u}$ and $\mu \nabla^{2} \vec{u}$ can be derived using the typical speed, time, and length scale of the flow (Eq. 2.2). The Reynolds number is hence defined as

$$
\mathcal{R} \equiv \frac{\text { inertial force }}{\text { viscous force }}=\frac{\rho U^{2} / L}{\mu U / L^{2}}=\frac{\rho U L}{\mu}=\frac{U L}{\nu}
$$

where $\nu=\mu / \rho$ is the kinematic viscosity. The importance of the Reynolds number has been beautifully described by Purcell (1977). He also gives estimates for the order of magnitude of the Reynolds number: A human swimming in water experiences $\mathcal{R} \sim 10^{4}$, while for a goldfish it is $\mathcal{R} \sim 10^{2}$. However, microswimmers (typical length $1 \mu \mathrm{m}$, typical speed $30 \mu \mathrm{m} / \mathrm{s}$ ) in water (kinematic viscosity $\nu \approx 10^{-6} \mathrm{~m}^{2} / \mathrm{s}$ ) are subject to a Reynolds number of $\mathcal{R} \sim 3 \times 10^{-5}$. This means that their motion is completely dominated by the viscous force and inertia is negligible. By assuming $\mathcal{R} \ll 1$, Eq. (2.3) simplifies and yields the famous Stokes equation

$$
\begin{aligned}
0 & =\vec{f}^{\prime}-\nabla^{\prime} p^{\prime}+\nabla^{\prime 2} \vec{u}^{\prime} \\
\Rightarrow 0 & =\vec{f}-\nabla p+\mu \nabla^{2} \vec{u} .
\end{aligned}
$$

In physical terms this equation describes a flow where the viscosity is large compared to typical speed and length. The Stokes flow can be solved by the method of Green's functions, and the solution is called "Stokeslet" (Hancock, 1953). The Green's function is obtained by replacing the external force density by a point force at position $\vec{r}^{\prime}$ with strength $\overrightarrow{f_{0}}$ mathematically described by a Dirac delta distribution (Dhont, 1996, p. 241)

$$
\vec{f}(\vec{r})=\overrightarrow{f_{0}} \delta\left(\vec{r}-\vec{r}^{\prime}\right)
$$

The resulting flow velocity can be written as

$$
\vec{u}(\vec{r})=\mathcal{O}\left(\vec{r}-\vec{r}^{\prime}\right) \cdot \overrightarrow{f_{0}}
$$

with the so-called Oseen tensor $\mathcal{O}(\vec{r})$. The latter can be calculated and yields (Dhont, 1996, p. 243)

$$
\mathcal{O}(\vec{r})=\frac{1}{8 \pi \mu r}\left[\mathbf{I}+\frac{\vec{r} \otimes \vec{r}}{r^{2}}\right] .
$$

This fundamental solution of the Stokes equation is very useful because the response of the system to an arbitrary external force $\vec{f}(\vec{r})$ can be calculated from the superposition principle because the Stokes equation is linear (Dhont, 1996, p. 242):

$$
\vec{u}(\vec{r})=\int \mathcal{O}\left(\vec{r}-\vec{r}^{\prime}\right) \cdot \vec{f}\left(\vec{r}^{\prime}\right) \mathrm{d} \vec{r}^{\prime}
$$




\subsubsection{Microswimmmer: Force dipole}

The Oseen tensor can be used to calculate the resulting flow field due to any external force. We aim to derive the hydrodynamic interactions between microswimmers. Hence we need a description of the microswimmers as a force field. It is very common to describe the force field far from the microswimmer as a static force dipole (Lighthill, 1975; Ishikawa et al., 2006). The swimmer is modeled (Baskaran and Marchetti, 2009) as a rigid dumbbell with two differently sized spheres (radii $a_{S}, a_{L}$ ) connected by an infinitely thin rod along the unit vector $\hat{e}$. The distance between the centers of the spheres is given by $l$. The swimmer exerts equal and opposite forces onto the fluid at both ends parallel to the connecting rod. The self-propulsion of the microswimmer originates from the swimmer's asymmetry which results in a shift of the hydrodynamic center away from the geometric center of the dumbbell. The speed of the swimmer is proportional to the strength $f$ of the force dipole as $v_{0}=-f\left(a_{L}-a_{S}\right) /\left(4 \pi \mu l\left(a_{L}+a_{S}\right)\right)$. The sign of $f$ determines whether a swimmer is a pusher or a puller.

The external force density in the Stokes equation of the ambient fluid consists of two parts: The active force density and the force density due to fluctuations in the fluid, $\vec{f}=\vec{f}_{\text {active }}-\vec{f}_{\text {noise }}$, with

$$
\begin{aligned}
\vec{f}_{\text {active }} & =\sum_{\alpha=1}^{N} f \hat{e}_{\alpha}\left[\delta\left(\vec{r}-\vec{r}_{L \alpha}\right)-\delta\left(\vec{r}-\vec{r}_{S \alpha}\right)\right] \text { and } \\
\vec{f}_{\text {noise }} & =\sum_{\alpha=1}^{N}\left[\xi_{\alpha}^{L}(t) \delta\left(\vec{r}-\vec{r}_{L \alpha}\right)+\xi_{\alpha}^{S}(t) \delta\left(\vec{r}-\vec{r}_{S \alpha}\right)\right] .
\end{aligned}
$$

The sum extends to the $N$ particles in the system and $\xi_{\alpha}^{L, S}$ are random forces to account for thermal diffusion of the spheres. The resulting Stokes equation (Eq. 2.6) can formally be solved by the Oseen tensor because the forces are point-like. The solution is then inserted into the equations of motion of the swimmer which identify the velocity of the fluid at the position of each sphere with the velocity of that sphere:

$$
\begin{aligned}
\frac{\partial \vec{r}_{L \alpha}}{\partial t} & =\vec{u}\left(\vec{r}_{L \alpha}\right) \text { and } \\
\frac{\partial \vec{r}_{S \alpha}}{\partial t} & =\vec{u}\left(\vec{r}_{S \alpha}\right) .
\end{aligned}
$$

Expressions for the forces and torques exerted on each swimmer due to the presence of the other swimmers can be derived by a multipole expansion (Baskaran and Marchetti, 2009, supplementary material). The torque which one particle exerts on 
another can be written (in a simplified manner) as

$$
\vec{\tau}_{12} \simeq \frac{f\left(a_{L}+a_{S}\right)}{8} \vec{e}_{1} \times\left[3 \hat{r}_{12} \otimes \hat{r}_{12}-\mathbf{I}\right] \cdot \hat{e}_{2}\left[\frac{l^{3}}{r_{12}^{3}}\left(\hat{e}_{1} \cdot \hat{e}_{2}\right)-\frac{2\left(a_{L}-a_{S}\right)^{2}}{\left(a_{L}+a_{S}\right)^{2}} \frac{l^{5}}{r_{12}^{5}}\right]
$$

where $\vec{r}_{12}=r_{12} \hat{r}_{12}$ is the vector connecting the two hydrodynamic centers of the swimmers. The torque consists of two terms. The first term is of the order of $\left(l / r_{12}\right)^{3}$ and leads to nematic alignment because it is invariant under the inversion of $\hat{e}_{1}$ or $\hat{e}_{2}$. The second term (of the order of $\left.\left(l / r_{12}\right)^{5}\right)$ leads to polar alignment. It vanishes in the case of a symmetric dumbbell which is active (because it leads to a fluid motion) but at the same time is not self-propelled $\left(v_{0}=0\right)$. This limiting case of the dumbbell model is called a "shaker" because of the resulting motion due to thermal fluctuations. The hydrodynamic interaction of microswimmers is hence of nematic symmetry to leading order which allows us to use simple nematic interaction rules in our model so that the far field hydrodynamic interactions between active particles are implicitly incorporated at the first order. We can compare the magnitude of both terms to estimate the validity of pure nematic interaction:

$$
\frac{\mid \text { nematic alignment } \mid}{\mid \text { polar alignment } \mid}=\frac{l^{3}}{r_{12}^{3}} \frac{\left(a_{L}+a_{S}\right)^{2}}{2\left(a_{L}-a_{S}\right)^{2}} \frac{r_{12}^{5}}{l^{5}}=\frac{1}{2}\left(\left[\frac{a_{L}+a_{S}}{a_{L}-a_{S}}\right] \frac{r_{12}}{l}\right)^{2} .
$$

Hence if the two spheres of the dumbbell are similar in size, the nematic term dominates the system considerably more than for a very asymmetric dumbbell.

\subsection{Overdamped dynamics}

The Langevin equation is a stochastic differential equation which describes the change in momentum of a micron-scaled object immersed in a fluid. It was introduced to describe Brownian motion (Langevin, 1908), i.e. the motion of one big, heavy particle in a bath of small and light particles. The Langevin equation describes the motion of the large particle (position $\vec{x}$, mass $m=1$ ) while the influence of the small particle is mimicked by a random force which models the thermal motion of the fluid molecules. In general, the evolution equation of the particle velocity $\vec{v}=\mathrm{d} \vec{x} / \mathrm{d} t$ is then given by

$$
\frac{\mathrm{d} \vec{v}}{\mathrm{~d} t}+\gamma \vec{v}=\vec{\Gamma}(t)
$$

(Risken, 1989, p. 32). The force acting on the particle is hence balanced by a friction force (with friction coefficent $\gamma$ ) and a random Langevin force $\vec{\Gamma}(t)$ which has zero mean and is delta-correlated in time. For interacting particles, the Langevin equation can be modified by an additional potential term $-\mathrm{d} V(\vec{x}) / \mathrm{d} \vec{x}$ on the right-hand side of Eq. (2.15). 
In the low-Reynolds number regime (which is valid for microswimmers, see Section 2.1), the dynamics of the particle is overdamped because the viscous force dominates over inertia. This means that accelerations are damped out very quickly so that we can neglect the first term on the left-hand side of Eq. (2.15). The resulting overdamped Langevin equation with interactions is given by

$$
\frac{\mathrm{d} \vec{x}}{\mathrm{~d} t}=-\frac{1}{\gamma} \frac{\mathrm{d} V(\vec{x})}{\mathrm{d} \vec{x}}+\frac{1}{\gamma} \vec{\Gamma}(t)
$$

\subsection{Turbulence}

We want to study active particles in a turbulent flow field and therefore we introduce a few basic concepts of turbulence in this section: The energy-spectrum function, the integral length scales of velocity and vorticity, and the Taylor-based Reynolds number. Generally, turbulence describes a stochastic flow regime which is found in many different fluids and situations. It occurs at high Reynolds numbers, i.e., when the influence of viscosity is small compared to the importance of inertia. It is important to note that this is not the same Reynolds number as the one describing the motion of the microswimmer: The swimmer's Reynolds number characterizes its motion in a fluid based on the swimmer's size and speed, and the viscosity of the fluid. The (potentially high) Reynolds number of the turbulent flow field instead is an intrinsic property of that flow.

The main quantities of interest about the turbulent field are the velocity $\vec{u}_{\text {turb }}(\vec{r}, t)$, the pressure, and the density which are all coupled through the (incompressible) Navier-Stokes equations (Eq. 2.1). From the turbulent velocity it is easy to calculate the vorticity $\vec{\omega} \equiv \nabla \times \vec{u}_{\text {turb }}$ which is responsible for the rotation of fluid elements (and also immersed particles).

\subsubsection{Energy spectrum}

Turbulent flows consist of structures which span a wide range of sizes. They are often characterized by their energy-spectrum function which describes the distribution of energy among the differently sized structures. In the following we sketch how this function can be derived from the velocity field of a homogeneous and isotropic flow. We therefore start with the two-point velocity correlation function which is given by

$$
R_{i j}(\vec{r}, t) \equiv\left\langle u_{i}(\vec{x}+\vec{r}, t) u_{j}(\vec{x}, t)\right\rangle
$$

(Pope, 2000, p. 196) where $\langle\cdot\rangle$ denotes an average over positions $\vec{x} . \quad R_{i j}$ does not depend on $\vec{x}$ because the turbulence is (assumed to be) homogeneous. We will drop the time-dependence in the following because we are only interested in stationary 
turbulence. The velocity-spectrum tensor is the Fourier transform of the velocity correlation:

$$
\Phi_{i j}(\vec{k}) \equiv \frac{1}{(2 \pi)^{3}} \iiint_{-\infty}^{\infty} R_{i j}(\vec{r}) \mathrm{e}^{-i \vec{k} \cdot \vec{r}} \mathrm{~d} \vec{r}
$$

(Pope, 2000, p. 220). The energy spectrum function can be defined by removing all orientational information from the velocity-spectrum tensor. To achieve this, one uses only the trace of the tensor and integrates over a sphere of radius $k$ :

$$
E(k) \equiv \oint_{S} \frac{1}{2} \Phi_{i i}(\vec{k}) \mathrm{d} S(k)
$$

(Pope, 2000, p. 221). In isotropic turbulence, it can be shown that the velocityspectrum tensor is completely determined by the energy-spectrum function:

$$
\Phi_{i j}(\vec{k})=\frac{E(k)}{4 \pi k^{2}}\left(\delta_{i j}-\frac{k_{i} k_{j}}{k^{2}}\right)
$$

(Pope, 2000, p. 222).

The turbulent kinetic energy is given by the integral over the energy-spectrum function

$$
k \equiv \int E(k) \mathrm{d} k
$$

and measures the full kinetic energy of the turbulent flow.

Kolmogorov spectrum- Kolmogorov (1941) introduced hypotheses on turbulence of high enough Reynolds number which lead to an energy-spectrum function $E(k)$ (Pope, 2000 , p. 230). This energy-spectrum function describes how energy is distributed to different length scales in the system (described by the wavenumber $k$ ). The first hypothesis states that turbulence should obey local isotropy. The second hypothesis is a statement on the universal form of the velocity statistics of the small-scale motions which is uniquely determined by the energy dissipation rate $\bar{\epsilon}$ and the kinematic viscosity $\nu$. Dimensional analysis leads to the resulting energy-spectrum function in the inertial subrange as

$$
E(k)=C \bar{\epsilon}^{2 / 3} k^{-5 / 3}
$$

with a universal Kolmogorov constant $C$.

Typical velocity and vorticity. The turbulent field is described by the energyspectrum function. However, it is useful to derive typical values of the velocity and vorticity of the turbulent field to compare to other values in the system. The typical 
velocity is the root-mean-square velocity:

$$
u_{\mathrm{rms}} \equiv \sqrt{\left\langle\vec{u}_{\text {turb }}^{2}\right\rangle} .
$$

It is related to the turbulent kinetic energy $k$ as

$$
k=\frac{1}{2} u_{\mathrm{rms}}^{2}
$$

and can hence also be calculated from the energy-spectrum function

$$
u_{\mathrm{rms}}=\sqrt{2 k}=\sqrt{2 \int_{0}^{\infty} E(k) \mathrm{d} k} .
$$

It is important to note here that sometimes in the literature the term "root-meansquare velocity" refers to the root-mean-square value of one component of the velocity vector, which in isotropic turbulence is a third of our $u_{\mathrm{rms}}$.

Like the energy-spectrum function is based on the velocity, a similar function can be derived for the vorticity as well. It can even be shown that such a spectrum of the vorticity is related to the energy-spectrum function by

$$
E_{\omega}(k)=k^{2} E(k) .
$$

In a flow field which obeys the Kolmogorov spectrum, the large-scale structures possess most of the kinetic energy due to the negative slope of $E(k)$. The spectrum of the vorticity tells us, however, that in terms of rotations (which is what the vorticity is responsible for) the small-scale structures dominate due to the positive slope of $E_{\omega}(k)$. The root-mean-square vorticity is given (in complete analogy to $u_{\text {rms }}$ ) by

$$
\omega_{K}=\sqrt{2 \int_{0}^{\infty} E_{\omega}(k) \mathrm{d} k} .
$$

In the following, it will be called the "Kolmogorov shear rate" (Durham et al., 2013).

\subsubsection{Integral length scale}

It is useful to calculate a typical length scale of the turbulent flow field to be able to compare it to other length scales in the system. Usually in isotropic turbulence one calculates the longitudinal integral length scale of the velocity field which is defined for isotropic turbulence as the space integral over the correlation of a velocity component 
(called $u_{1}$ ) correlated along the $\hat{e}_{1}$-axis:

$$
L_{11}(t) \equiv \int_{0}^{\infty} \frac{\left\langle u_{1}\left(\vec{x}+\hat{e}_{1} r, t\right) u_{1}(\vec{x}, t)\right\rangle}{\left\langle u_{1}^{2}\right\rangle} \mathrm{d} r
$$

(Pope, 2000, p. 197). We will omit the time dependence hereinafter because we only study statistically stationary turbulence. Using the one-dimensional energy spectrum $E_{11}\left(k_{1}\right)=2 \iint_{-\infty}^{\infty} \Phi_{11}(\vec{k}) \mathrm{d} k_{2} \mathrm{~d} k_{3}$ together with the relationship between velocityspectrum tensor and turbulent energy spectrum function $E(k)$ (Eq. 2.20) leads to

$$
L_{11}=\frac{3 \pi}{4} \frac{\int_{0}^{\infty} E(k) k^{-1} \mathrm{~d} k}{\int_{0}^{\infty} E(k) \mathrm{d} k} .
$$

Analogously to Eq. (2.28) one can also define a longitudinal integral length scale of the vorticity:

$$
L_{11}^{\omega} \equiv \int_{0}^{\infty} \frac{\left\langle\omega_{1}\left(\vec{x}+\hat{e}_{1} r\right) \omega_{1}(\vec{x})\right\rangle}{\left\langle\omega_{1}^{2}\right\rangle} \mathrm{d} r .
$$

Again, we omit the time dependence of $L_{11}^{\omega}$ because of statistical stationarity. In the following we derive an expression for $L_{11}^{\omega}$ in complete analogy to the derivation of Eq. (2.29). We start from the two-point vorticity correlation

$$
R_{i j}^{\omega}(\vec{r}) \equiv\left\langle\omega_{i}(\vec{x}+\vec{r}) \omega_{j}(\vec{x})\right\rangle
$$

which in the case of isotropic turbulence can be decomposed into two scalar functions: the longitudinal and transverse autocorrelation functions (cf. Pope, 2000, p. 196). We use the longitudinal autocorrelation function

$$
f_{\omega}(r)=\frac{R_{11}^{\omega}\left(\hat{e}_{1} r\right)}{\left\langle u_{1}^{2}\right\rangle}
$$

which leads to

$$
L_{11}^{\omega}=\int_{0}^{\infty} f_{\omega}(r) \mathrm{d} r
$$

The one-dimensional spectra $E_{i j}^{\omega}\left(k_{1}\right)$ are defined as

$$
E_{i j}^{\omega}\left(k_{1}\right) \equiv \frac{1}{\pi} \int_{-\infty}^{\infty} R_{i j}^{\omega}\left(\hat{e}_{1} r\right) \mathrm{e}^{-i k_{1} r} \mathrm{~d} r .
$$


By using Eq. (2.32) and the properties of $f_{\omega}$, as well as Eq. (2.33), we find

$$
\begin{aligned}
E_{11}^{\omega}\left(k_{1}\right) & =\frac{2}{\pi}\left\langle u_{1}^{2}\right\rangle \int_{0}^{\infty} f_{\omega}(r) \cos \left(k_{1} r\right) \mathrm{d} r \\
\Rightarrow L_{11}^{\omega} & =\int_{0}^{\infty} f_{\omega}(r) \mathrm{d} r=\frac{\pi}{2} \frac{E_{11}^{\omega}(0)}{\left\langle\omega_{1}^{2}\right\rangle}
\end{aligned}
$$

Hence, we need expressions for the one-dimensional spectrum $E_{11}\left(k_{1}\right)$ and for the mean vorticity $\left\langle\omega_{1}^{2}\right\rangle$ to calculate the integral length scale. The one-dimensional spectrum is related to the vorticity-spectrum tensor $\Phi_{i j}^{\omega}$

$$
\Phi_{i j}^{\omega}(\vec{k}) \equiv \frac{1}{(2 \pi)^{3}} \iint_{-\infty}^{\infty} \int_{i j}^{\omega}(\vec{r}) \mathrm{e}^{-i \vec{k} \cdot \vec{r}} \mathrm{~d} \vec{r}
$$

(defined as the Fourier transform of Eq. 2.31) via

$$
E_{11}^{\omega}\left(k_{1}\right)=2 \iint_{-\infty}^{\infty} \Phi_{11}^{\omega}(\vec{k}) \mathrm{d} k_{2} \mathrm{~d} k_{3}
$$

The vorticity-spectrum tensor is in turn related to the velocity-spectrum tensor $\Phi_{i j}$ (Eq. 2.18) as

$$
\Phi_{i j}^{\omega}=\left(\delta_{i j} k^{2}-k_{i} k_{j}\right) \Phi_{k k}-k^{2} \Phi_{j i}
$$

(Saffman, 1967) and the velocity-spectrum tensor is given by the energy-spectrum function in Eq. (2.20). This leads to

$$
\begin{aligned}
\Phi_{11}^{\omega} & =\frac{E(k)}{4 \pi}\left(1-\frac{k_{1}^{2}}{k^{2}}\right) \mathrm{d} k_{2} \mathrm{~d} k_{3} \\
\Rightarrow E_{11}^{\omega}\left(k_{1}\right) & =2 \iint_{-\infty}^{\infty} \frac{E(k)}{4 \pi}\left(1-\frac{k_{1}^{2}}{k^{2}}\right) \mathrm{d} k_{2} \mathrm{~d} k_{3} .
\end{aligned}
$$

We use spherical coordinates $k_{r}^{2}=k_{2}^{2}+k_{3}^{2}=k^{2}-k_{1}^{2}$ and the fact that $k_{r} \mathrm{~d} k_{r}=k \mathrm{~d} k$ (Pope, 2000, p. 226) which leads to

$$
\begin{aligned}
E_{11}^{\omega}\left(k_{1}\right) & =2 \int_{0}^{2 \pi} \int_{0}^{\infty} \frac{E(k)}{4 \pi}\left(1-\frac{k_{1}^{2}}{k^{2}}\right) k_{r} \mathrm{~d} k_{r} \mathrm{~d} \phi \\
& =\int_{0}^{\infty} E(k)\left(1-\frac{k_{1}^{2}}{k^{2}}\right) k \mathrm{~d} k \\
\Rightarrow E_{11}^{\omega}(0) & =\int_{0}^{\infty} E(k) k \mathrm{~d} k .
\end{aligned}
$$


The mean vorticity $\left\langle\omega_{1}^{2}\right\rangle$ can be calculated from the vorticity spectrum (Eq. 2.26) as

$$
\left\langle\omega_{1}^{2}\right\rangle=\frac{1}{3} \omega_{K}^{2}=\frac{1}{3} \int_{0}^{\infty} E(k) k^{2} \mathrm{~d} k .
$$

By combining Eq. (2.36) with Eqns. (2.44) and (2.45), the integral length scale is given by

$$
L_{11}^{\omega}=\frac{3 \pi}{4} \frac{\int_{0}^{\infty} E(k) k \mathrm{~d} k}{\int_{0}^{\infty} E(k) k^{2} \mathrm{~d} k}
$$

\subsubsection{Taylor-based Reynolds number}

In general, the Reynolds number of any flow field is defined as the ratio of inertial over viscous forces and can be derived as $\mathcal{R}=U L / \nu$ from a typical speed $U$, a typical length $L$, and the kinematic viscosity $\nu$ (see Eq. 2.5 and derivation thereof). In the turbulence community it is common to use as a typical speed the root-mean-square speed $u^{\prime}$ based on one component of the turbulent flow field. This is related to the full root-mean-square velocity and to the turbulent kinetic energy (see Eq. 2.24) as

$$
u^{\prime 2}=\frac{1}{3} u_{\mathrm{rms}}^{2}=\frac{2}{3} k .
$$

The length scales involved in a turbulent flow field span a wide range and therefore we can define different Reynolds numbers based on different "typical" length scales of the flow field. The typical length scales of the large-scale eddies can be estimated by the length $L=k^{3 / 2} / \bar{\epsilon}$ with the energy dissipation rate $\bar{\epsilon}$ so that the turbulence Reynolds number can be defined as

$$
\mathcal{R}_{L} \equiv \frac{\sqrt{k} L}{\nu}=\frac{k^{2}}{\bar{\epsilon} \nu}
$$

(see Pope, 2000, p. 200).

A second Reynolds number which characterizes the turbulent flow field is based on the Taylor microscale $\lambda_{g}$. This scale does not have a clear physical interpretation (Pope, 2000, p. 199) but it is very useful to characterize a turbulent flow field. For example it is related to the energy dissipation rate and the viscosity through

$$
\bar{\epsilon}=15 \nu \frac{u^{\prime 2}}{\lambda_{g}^{2}}
$$

(Pope, 2000, p. 199), an expression which was first derived by Taylor (1935). In his classic paper, he incorrectly concluded that the Taylor microscale characterizes the size at which the energy dissipated. This is not true because it erroneously assumes 
that $u^{\prime}$ is the corresponding characteristic velocity of those smallest eddies. The Taylor-based Reynolds number is defined as

$$
\mathcal{R}_{\lambda} \equiv \frac{u^{\prime} \lambda_{g}}{\nu}
$$

which leads to

$$
\mathcal{R}_{\mathrm{\lambda}}=\sqrt{\frac{20}{3} \frac{k^{2}}{\nu \bar{\epsilon}}}=\sqrt{\frac{20}{3} \mathcal{R}_{L}} .
$$

Even though the physical interpretation of the Taylor microscale is not straightforward, the resulting Reynolds number does have a physical interpretation: It is equal to the ratio of the large-eddy time scale to the time-scale of fluctuations in the strain rate (Tennekes and Lumley, 1972, p. 68). 


\section{Methods}

In this Chapter the methods are introduced which we use to study self-propelled particles with and without an external turbulent field. We describe the equations of motion of the self-propelled particles, as well as the models of the turbulent flow field. The numerical implementation is discussed. We introduce dimensionless numbers and order parameters to describe our system and briefly describe different methods to analyze clustering.

\subsection{Models}

The subject of this Section are the different models which we study in this thesis: point-like, self-propelled particles; extended, self-propelled particles; and selfpropelled particles in a turbulent field. The turbulent flow field is either generated from kinematic simulations (called the "Kraichnan flow field" in the following) or using direct numerical simulations.

\subsubsection{Nematically aligning self-propelled particles}

We investigate the collective behavior of large numbers of SPPs in terms of MD simulations. To this end $N$ particles inhabit a three-dimensional rectangular box of side lengths $L_{x}, L_{y}, L_{z}$. Most of the simulations are performed in a cubic box and the linear size $L=L_{x}=L_{y}=L_{z}$ is given. If not stated otherwise, periodic boundary conditions are applied in all three dimensions. The particle $i$ is described by its position $\vec{r}_{i}$ and its orientation $\hat{e}_{i}$ with $\hat{e}_{i}^{2}=1$. It moves along this orientation vector 
with a constant speed $v_{0}$ which makes the particle self-propelled. Neighboring particles within a distance $\epsilon$ interact nematically, i.e. they tend to align their orientations while ignoring the positions of head and tail. This interaction is incorporated into the model by means of the Lebwohl-Lasher potential (Lebwohl and Lasher, 1972) which is commonly used in the field of liquid crystals to simulate nematic interactions. The potential reads as follows:

$$
U_{\mathrm{LL}} \equiv-\frac{1}{2} \sum_{i}^{N} \frac{1}{n_{i}} \sum_{j \in n_{i}}\left(\hat{e}_{i} \cdot \hat{e}_{j}\right)^{2}
$$

where the first sum extends over all simulated particles while the second sum extends to the $n_{i}$ neighboring particles of particle $i$ within a sphere of radius $\epsilon$. Finally, the orientation of the particles are subject to an additive stochastic noise (details will be given below). The equations of motion for particle $i$ are thus

$$
\begin{aligned}
& \dot{\vec{r}}_{i}=v_{0} \hat{e}_{i}=\vec{v}_{i} \\
& \dot{\hat{e}}_{i}=\left[-\gamma \frac{\partial U_{\mathrm{LL}}}{\partial \hat{e}_{i}}+\vec{\xi}_{i}\right]_{\perp}=\left[\vec{T}_{i}\right]_{\perp}
\end{aligned}
$$

with the relaxation constant $\gamma$ and the stochastic noise $\hat{\xi}_{i}$. Eq. (3.2) means physically that particle $i$ moves with a velocity $\vec{v}_{i}$ and rotates subject to the component of the torque $\vec{T}_{i}$ which is perpendicular to the particle orientation as indicated by the subscript $\perp$ :

$$
\left[\vec{T}_{i}\right]_{\perp} \equiv \vec{T}_{i}-\left(\vec{T}_{i} \cdot \hat{e}_{i}\right) \hat{e}_{i}
$$

The equations are only first order in time because we work in the limit of low Reynolds numbers which means that viscous effects dominate over inertia and accelerations are damped out very fast (in the spirit of the Langevin equation in the overdamped limit, see Section 2.2). The nematic symmetry of the interaction is justified by the fact that the hydrodynamic interactions between swimmers (modeled as force dipoles) are nematic to leading order (see Section 2.1 and Baskaran and Marchetti, 2009, for details).

\subsubsection{Particles with hard cores}

It is very common in MD simulations to model extended particles by the WCA potential. This potential was introduced by Weeks et al. (1971) and consists of a shifted and truncated Lennard-Jones potential (Lennard-Jones, 1931). The resulting 


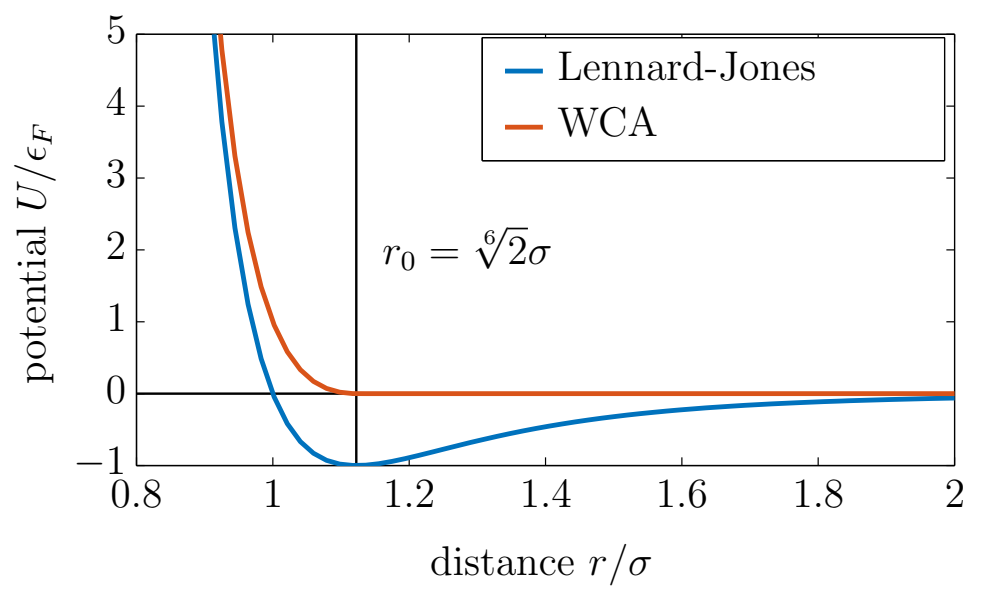

Figure 3.1: Illustration of the WCA potential (red). The Lennard-Jones potential (blue) is shifted by $\epsilon_{F}$ and truncated at $r_{0}=\sqrt[6]{2} \sigma$.

potential is purely repulsive and can be written as

$$
U_{\mathrm{WCA}}(r) \equiv \begin{cases}4 \epsilon_{F}\left(\left(\frac{\sigma}{r}\right)^{12}-\left(\frac{\sigma}{r}\right)^{6}\right)+\epsilon_{F} & \text { for } r \leq \sqrt[6]{2} \sigma \\ 0 & \text { for } r>\sqrt[6]{2} \sigma\end{cases}
$$

with $\epsilon_{F}$ being the strength of the potential. The particle diameter is denoted by $\sigma$ (see Fig. 3.1 for an illustration of the potential).

The resulting force $\vec{F}_{\mathrm{WCA}}=-\nabla U_{\mathrm{WCA}}$ enters the equation of the particle position together with the relaxation time $\gamma_{F}$ (Wysocki et al., 2014). Moreover, as the model is supposed to mimic elongated objects, also the orientation of the particle is altered nematically. The resulting equations of motion are

$$
\begin{aligned}
& \dot{\vec{r}}_{i}=v_{0} \hat{e}_{i}+\frac{1}{\gamma_{F}} \sum_{j \neq i} \vec{F}_{\mathrm{WCA}}=v_{0} \hat{e}_{i}-\frac{1}{\gamma_{F}} \sum_{j \neq i} \nabla_{i} U_{\mathrm{WCA}}\left(r_{i j}\right)=\vec{v}_{i}^{\mathrm{WCA}}, \\
& \dot{\hat{e}}_{i}=\left[-\gamma \frac{\partial U_{\mathrm{LL}}}{\partial \hat{e}_{i}}+\vec{\xi}_{i}-\sum_{j \neq i} \operatorname{sign}\left(\hat{e}_{i} \cdot \hat{e}_{j}\right) \nabla_{i} U_{\mathrm{WCA}}\left(r_{i j}\right)\right]_{\perp}=\left[\vec{T}_{i}^{\mathrm{WCA}}\right]_{\perp},
\end{aligned}
$$

where $r_{i j}=\left|\vec{r}_{i}-\vec{r}_{j}\right|$ and

$$
\operatorname{sign}(x) \equiv \begin{cases}+1 & \text { for } x>0 \\ 0 & \text { for } x=0 \\ -1 & \text { for } x<0\end{cases}
$$




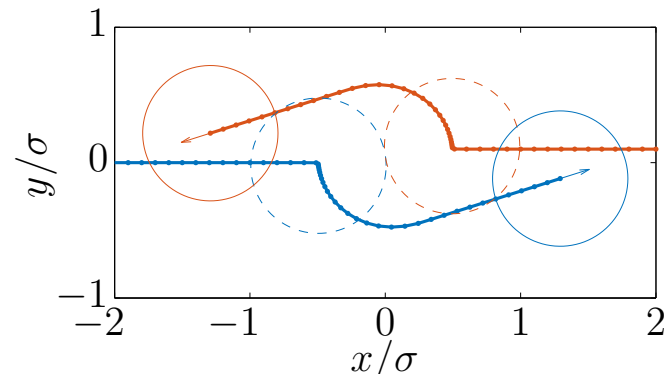

(a) Head-on collision with influence of the WCA potential on the particle orientation following Eq. (3.5)

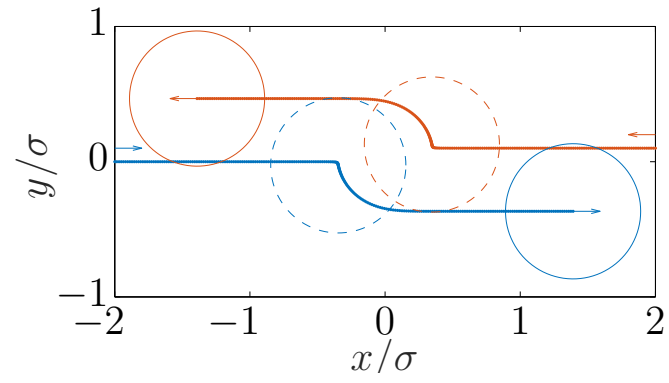

(b) Head-on collision without influence of the WCA potential on the particle orientation following Eq. (3.8) with $E_{0}=0$.

Figure 3.2: Illustration of the effect of hard cores modeled through the WCA force $(\mathscr{P} \rightarrow \infty)$. Two particles approach each other along $\hat{x}$ while they are $\Delta y=0.1 \sigma$ apart (blue: moving along $\hat{x}$, red: moving along $-\hat{x}$ ). The circles denote the particle size, the dashed circles are drawn for the shortest distance between the two particles.

The trajectories of two particles which follow these equations of motion are illustrated in Fig. 3.2a. They collide head-on starting from initial positions with a small shift perpendicular to the axis of their orientations. Note that the particles repel each other and also influence each others orientation thus resulting in a rotation of the overall nematic orientation.

\subsubsection{Particles in a turbulent field}

The collective behavior of particles immersed in a turbulent field will be studied in Chapter 6. This flow field influences the particles in two different ways (Durham et al., 2013): Firstly, the particles are advected by the turbulent velocity $\vec{u}_{\text {turb }}$. And secondly, the particles are turned by the turbulent vorticity $\vec{\omega}=\nabla \times \vec{u}_{\text {turb }}$ which means that the turbulent vorticity acts as a torque on the orientation of the particles. It can be shown that the magnitude of the angular velocity of a fluid parcel is half of the magnitude of the vorticity. The resulting equations of motion for the self-propelled particles are (analogously to Eq. 3.2):

$$
\begin{array}{ll}
\dot{\vec{r}}_{i}=v_{0} \hat{e}_{i}+\vec{u}_{\text {turb }} & =\vec{v}_{i}^{\text {turb }}, \\
\dot{\hat{e}}_{i}=\left[-\gamma \frac{\partial U_{\mathrm{LL}}}{\partial \hat{e}_{i}}+\frac{1}{2}\left(\vec{\omega}\left(\vec{r}_{i}, t\right) \times \hat{e}_{i}\right)+\vec{\xi}_{i}\right]_{\perp}=\left[\vec{T}_{i}^{\text {turb }}\right]_{\perp} .
\end{array}
$$

The turbulent field is introduced in two different manners: One uses the method of "kinematic simulation" by Kraichnan (1970) which mimics the turbulent field by a Fourier sum satisfying certain conditions such as incompressibility and a prescribed 
energy spectrum. Hereinafter this method will be referred to as the 'Kraichnan method' (see Section 3.1.4 for details). The second method uses DNSs as described in Section 3.1.5.

\section{Extended particles in a turbulent field}

We also want to model hard-core particles in a turbulent field. The idea is similar to hard-core particles without a turbulent field but we omit the influence of the WCA force onto the orientation of the particles to keep the model as simple as possible and ensure a direct comparability between the models with point particles and with hard cores. The resulting equations of motions are (cf. Eqns. 3.7 and 3.5):

$$
\begin{aligned}
& \dot{\vec{r}}_{i}=v_{0} \hat{e}_{i}+\vec{u}_{\mathrm{turb}}-\frac{1}{\gamma_{F}} \sum_{j \neq i} \nabla_{i} U_{\mathrm{WCA}}\left(r_{i j}\right)=\vec{v}_{i}^{\text {turb, } \mathrm{WCA}}, \\
& \dot{\hat{e}}_{i}=\left[-\gamma \frac{\partial U_{\mathrm{LL}}}{\partial \hat{e}_{i}}+\frac{1}{2}\left(\vec{\omega}\left(\vec{r}_{i}, t\right) \times \hat{e}_{i}\right)+\vec{\xi}_{i}\right]_{\perp}=\left[\vec{T}_{i}^{\text {turb, WCA }}\right]_{\perp} .
\end{aligned}
$$

The resulting motion of two particles which collide head-on with a small shift perpendicular to their axis of motion is illustrated in Fig. 3.2b. Now, the orientation of the particle is not altered by the WCA force and hence the global nematic director remains the same before and after the collision.

\subsubsection{The Kraichnan flow field}

Kraichnan (1970) developed a method to generate a flow field numerically which resembles a real turbulent field. The main advantage of this method in comparison to DNSs is that it is computationally quite easy which results in short run times even for rather large systems. Moreover, it does not require neither a space nor a time grid but it is continuous by construction. That means that the turbulent velocity and vorticity acting on any particle can be directly calculated at its position and at any time without requiring any interpolation. Finally, it follows a prescribed energy spectrum so that we are able to control the relative influences of large and small scales. The Kraichnan method has been used and developed further since its invention (Fung et al., 1992; Malik, 1996; Malik and Vassilicos, 1999; Visser and Jackson, 2004; Nicolleau et al., 2016) and we follow the recipe by Mariani et al. (2007) with modifications mainly concerning the possible wavevectors and -numbers. The turbulent field at position $\vec{r}$ and time $t$ is given by

$$
\vec{u}_{\text {turb }}(\vec{r}, t)=\sum_{n=1}^{N_{F}} c_{n}\left[\frac{\hat{a}_{n} \times \hat{k}_{n}}{\left|\hat{a}_{n} \times \hat{k}_{n}\right|} \cos \Omega_{n}(\vec{r}, t)+\frac{\hat{b}_{n} \times \hat{k}_{n}}{\left|\hat{b}_{n} \times \hat{k}_{n}\right|} \sin \Omega_{n}(\vec{r}, t)\right]
$$


and the vorticity follows as

$\vec{\omega}(\vec{r}, t)=\sum_{n=1}^{N_{F}} c_{n} k_{n}\left[-\hat{k}_{n} \times\left(\frac{\hat{a}_{n} \times \hat{k}_{n}}{\left|\hat{a}_{n} \times \hat{k}_{n}\right|}\right) \sin \Omega_{n}(\vec{r}, t)+\hat{k}_{n} \times\left(\frac{\hat{b}_{n} \times \hat{k}_{n}}{\left|\hat{b}_{n} \times \hat{k}_{n}\right|}\right) \cos \Omega_{n}(\vec{r}, t)\right]$.

The turbulent field is hence simulated by $N_{F}$ Fourier modes with weights $c_{n}$ and phases $\Omega_{n}(\vec{r}, t)=\vec{k}_{n} \vec{r}+\omega_{n} t$. The vectors $\vec{k}_{n}=k_{n} \hat{k}_{n}$ denote the wavevectors and $\omega_{n}$ are the corresponding angular frequencies. The vectors $\hat{a}_{n}$ and $\hat{b}_{n}$ are random vectors on a unit sphere. The velocity field is incompressible $\left(\nabla \cdot \vec{u}_{\text {turb }}=0\right)$ by construction due to the cross products which include $\hat{k}_{n}$.

In the MD simulations we apply full PBCs to our simulation box which is cubic with $L_{x}=L_{y}=L_{z}$. This implies that also the turbulent field itself has to fulfill the PBCs. This can be achieved by assuring that each individual mode fulfills the PBCs. To this end, the correct choice of the wavevectors is crucial: They have to be constructed as $\vec{k}_{n}=2 \pi \vec{n} / L$ with $\vec{n} \in \mathbb{N}^{3}$. The detailed procedure to find the wavevectors in the following: For each mode a random number $\chi \in\left[n_{\min }, n_{\max }\right)$ is generated as well as a random vector on the unit sphere. We multiply the two and round each component to the nearest integer. This leads to the vector $\vec{n} \in \mathbb{N}^{3}$, the direction of which is randomly distributed on the surface of a sphere. The vectors $\vec{n}$ are equally distributed in magnitude $|\vec{n}|$. All resulting vectors $\vec{n}$ are sorted by length and the wavevectors are calculated as $\vec{k}_{n}=2 \pi \vec{n} / L$ so that $k_{1}$ corresponds to the smallest wavenumber, while $k_{N_{F}}$ is the largest one. Several wavevectors can have the same magnitude and several wavevectors can point into the same direction but no pair of wavevectors must be identical.

By this procedure the wavenumbers $k_{n}$ are equally distributed. If this condition is relaxed and the vectors $\vec{n}$ are simply generated from $\vec{n} \in \mathbb{N}^{3}$ with $n_{\min } \leq|\vec{n}| \leq n_{\max }$ a few modes dominate the overall flow. This is because larger values of $|\vec{n}|$ are more probable and the small wavenumbers are very sparsely distributed because the probability of $|\vec{n}|$ is proportional to the volume of a spherical shell with radius $|\vec{n}|$ and thickness $\delta n$ and hence grows as $|\vec{n}|^{2}$. However, due to the negative slope of the energy-spectrum function, the modes with the smallest wavenumbers contain the largest amounts of energy. If they are very sparsely distributed then only a few modes contain most of the energy and dominate the overall flow pattern.

In the literature, other approaches to construct the wavevectors are used, especially a geometric series for the wavenumbers (Malik and Vassilicos, 1999; Lewis and Pedley, 2000; Visser and Jackson, 2004; Mariani et al., 2007). The resulting velocity field does not fulfill the periodic boundary conditions, so one could use "pseudo-periodic" boundary conditions (Mariani et al., 2007): To keep the concentration of particles constant, whenever a particle leaves the simulation domain a new one is created at random at the opposite side of the domain with a random orientation pointing into 
the simulation domain. A test of this approach implemented in our model showed that boundary effects still occurred, namely the local density at the boundary was increased compared to the inner part of the simulation domain. Since we want to study the clustering (i.e. local variations in density), we did not follow this approach further but used the described approach of a harmonic series of the wavevectors. A third possible choice of wavenumbers is to construct the wavevector from three independent Gaussian random number (Mansoori et al., 2002). Again, the resulting field will not meet the periodic boundary conditions and is hence not the right choice for our purpose.

Once we have found a set of wavevectors, we proceed by calculating the weights $c_{n}$ of the Fourier modes. The correlator of the turbulent velocity field is related to the energy spectrum $E(k)$ :

$$
\left\langle\vec{u}_{\text {turb }} \cdot \vec{u}_{\text {turb }}\right\rangle \stackrel{!}{=} 2 \int E(k) \mathrm{d} k,
$$

which is the definition of the energy spectrum for isotropic turbulence (Pope, 2000, p. 78). In the case of the Kraichnan model this auto-correlation is given by

$$
\left\langle\vec{u}_{\text {turb }} \cdot \vec{u}_{\text {turb }}\right\rangle=\sum_{n=1}^{N_{F}} c_{n}^{2}
$$

(Lewis and Pedley, 2000). A comparison between the latter and Eq. (3.11) leads to the idea to approximate the integral over the energy spectrum numerically by effectively assigning each mode to one segment of the numerical integral. As several wavevectors might have the same length, all $N_{F}$ wavevectors form $M$ equivalence classes $q_{1}, \ldots, q_{M}$. Each equivalence class $q_{m}=\left[k_{m}\right]$ contains $l_{m}$ wavevectors of length $k_{m}$. The weights of the equivalence classes must fulfill $2 \int E(k) \mathrm{d} k=\sum_{m=1}^{M}\left|c_{m}\right|^{2}$ which can be achieved by choosing $\left|c_{m}\right|^{2}=2 E\left(\left[k_{m}\right]\right) \delta\left[k_{m}\right]$ with $\delta\left[k_{m}\right]$ being the central difference between adjacent equivalence classes:

$$
\delta\left[k_{m}\right]=\frac{1}{2} \begin{cases}\left(\left[k_{2}\right]-\left[k_{1}\right]\right) & \text { for } m=1, \\ \left(\left[k_{m+1}\right]-\left[k_{m-1}\right)\right. & \text { for } 2 \leq m \leq M-1, \\ \left(\left[k_{M}\right]-\left[k_{M-1}\right]\right) & \text { for } m=M .\end{cases}
$$

Among each equivalence class, the energy is distributed equally:

$$
\left.c_{m}^{2}\left|=\sum_{j=1}^{l_{m}}\right| c_{\left[k_{m}\right]}\right|^{2}=l_{m}\left|c_{\left[k_{m}\right]}\right|^{2}
$$

which results in

$$
\left|c_{n}\right|=\frac{\left|c_{m}\right|}{\sqrt{l_{m}}}=\sqrt{\frac{2 E\left(\left[k_{m}\right]\right) \delta\left[k_{m}\right]}{l_{m}}} \quad, k_{n} \in\left[k_{m}\right]
$$


as the weight of each Fourier mode.

Finally, the angular frequency $\omega_{n}$ has to be chosen. Typically, the eddy turnover time is identified with $\omega_{n}$ which can be deduced from "Kolmogorov 1941 phenomenology" (see Frisch, 1995, p. 100-103): The eddy turnover time $t_{l}$ is the time it takes until a structure of size $l$ is significantly altered because of its relatively moving parts. This time scale can be approximated by $l$ and a typical velocity $v_{l}: t_{l} \sim l / v_{l}$. The energy flux is then given by $\Pi_{l}^{\prime} \sim v_{l}^{2} / t_{l} \sim v_{l}^{3} / l$. This energy flux is of the order of the dissipation rate $\bar{\epsilon}$ because the eddy turnover time also describes the time scale of the energy transfer from scales of the order of $l$ to smaller scales. This leads to

$$
\begin{aligned}
\Pi_{l}^{\prime} & \sim \bar{\epsilon} \\
\Rightarrow v_{l} & \sim \bar{\epsilon}^{1 / 3} l^{1 / 3} \\
\Rightarrow \frac{1}{t_{l}} & \sim \bar{\epsilon}^{1 / 3} l^{-2 / 3} \\
& =\sqrt{\bar{\epsilon}^{2 / 3} l^{-4 / 3}}=\sqrt{\bar{\epsilon}^{2 / 3}\left(\frac{1}{l}\right)^{-5 / 3}\left(\frac{1}{l}\right)^{3}}
\end{aligned}
$$

Using a Kolmogorov energy spectrum (Eq. 2.22) and identifying $k \sim l^{-1}$ leads to the following angular frequency

$$
\omega_{n}=0.4 \sqrt{E\left(k_{n}\right) k_{n}^{3}}
$$

with the unsteadiness parameter of 0.4 which was found to result in good agreement between DNS and kinematic simulation results (Malik and Vassilicos, 1999).

\section{Energy spectrum of the flow field}

To construct the Kraichnan flow field, we use an energy-spectrum function of the Kolmogorov type (Eq. 2.22):

$$
E(k)= \begin{cases}E_{0} k^{-5 / 3} & , \text { for } k_{\min } \leq k \leq k_{\max } \\ 0 & , \text { otherwise }\end{cases}
$$

This choice of $E(k)$ implies that we only model the inertial subrange. By comparing Eq. (3.18) with Eq. (2.22), we see that the energy scale $E_{0}$ effectively sets the dissipation rate $\bar{\epsilon}$. Changing $E_{0}$ does not change the range of wavenumbers (and hence does not change the Reynolds number) but only shifts the amount of energy each mode (and also the full field) contains. A possible extension of this energy spectrum would be to include exponential cut-offs (cf. Pope, 2000, p. 232).

The root-mean-square velocity (Eq. 2.25) and the Kolmogorov shear rate (Eq. 2.27) 
of a turbulent flow field with the given energy-spectrum function are given by

$$
\begin{aligned}
u_{\mathrm{rms}}^{\text {Kolmogorov }} & =\sqrt{3 E_{0}\left(k_{\min }^{-2 / 3}-k_{\max }^{-2 / 3}\right)}, \\
\omega_{K}^{\text {Kolmogorov }} & =\sqrt{\frac{3}{2} E_{0}\left(k_{\max }^{4 / 3}-k_{\min }^{4 / 3}\right)} .
\end{aligned}
$$

Also, the integral length scales of the velocity (Eq. 2.29) and of the vorticity (Eq. 2.46) follow from $E(k)$ :

$$
\begin{aligned}
L_{11}^{\text {Kolmogorov }} & =\frac{3 \pi}{10} \frac{1}{k_{\min }} \frac{1-\left(k_{\min } / k_{\max }\right)^{5 / 3}}{1-\left(k_{\min } / k_{\max }\right)^{2 / 3}}, \\
L_{11}^{\omega, \text { Kolmogorov }} & =\frac{3 \pi}{k_{\max }} \frac{1-\left(k_{\min } / k_{\max }\right)^{1 / 3}}{1-\left(k_{\min } / k_{\max }\right)^{4 / 3}} .
\end{aligned}
$$

\section{Taylor-based Reynolds number}

In the following we derive a formula for the Taylor-based Reynolds number of a kinematic simulation. This Reynolds number is given in Eq. (2.51) as a function of turbulent kinetic energy, kinematic viscosity, and energy dissipation rate. The turbulent kinetic energy (Eq. 2.21) of the Kolmogorov energy spectrum (Eq. 3.18) can be derived analytically as

$$
\begin{aligned}
k & =\int_{0}^{\infty} E(K) \mathrm{d} K=\int_{k_{\min }}^{k_{\max }} E_{0} K^{-5 / 3} \mathrm{~d} K \\
& =-\left.\frac{3}{2} E_{0} K^{-2 / 3}\right|_{k_{\min }} ^{k_{\max }} \\
& =\frac{3}{2} E_{0}\left(k_{\min }^{-2 / 3}-k_{\max }^{-2 / 3}\right) .
\end{aligned}
$$

We can choose the energy scale of the turbulent spectrum to be

$$
E_{0}=C \bar{\epsilon}^{2 / 3}\left(1-\left(\frac{k_{\min }}{k_{\max }}\right)^{4 / 3}\right)^{-1}
$$

to ensure the appropriate relation between the dissipation rate and the energy spectrum (see Eq. 2.22 and Mariani et al., 2007). For the moment, we keep the Kolmogorov constant $C$ variable. At the same time, the energy spectrum function also determines the energy dissipation rate of a turbulent flow (see Pope, 2000, p. 222):

$$
\bar{\epsilon}=2 \nu \int_{0}^{\infty} K^{2} E(K) \mathrm{d} K
$$


Inserting the Kolmogorov energy spectrum (Eq. 3.18) this leads to

$$
\bar{\epsilon}=\frac{3}{2} \nu E_{0}\left(k_{\max }^{4 / 3}-k_{\min }^{4 / 3}\right) .
$$

The kinematic viscosity can be derived from the definition of the Kolmogorov length scale (Tennekes and Lumley, 1972, p. 20)

$$
\eta_{K} \equiv\left(\frac{\nu^{3}}{\bar{\epsilon}}\right)^{1 / 4}
$$

so that the dissipation rate is given by

$$
\bar{\epsilon}^{2 / 3}=\frac{3}{2} \eta_{K}^{4 / 3} E_{0} k_{\max }^{4 / 3}\left(1-\left(\frac{k_{\min }}{k_{\max }}\right)^{4 / 3}\right) .
$$

We therefore have two equations (Eq. 3.23 and Eq. 3.27) which have both to be fulfilled by the Kolmogorov length scale $\eta_{K}$ and by the Kolmogorov constant $C$. The resulting conditions is

$$
1 \stackrel{!}{=} \frac{3}{2} C \eta_{K}^{4 / 3} k_{\max }^{4 / 3}
$$

Three different choices of $C$ and $\eta_{K}$ seem to be reasonable. Firstly, the smallest wavelength in the system is $2 \pi / k_{\max }$ which could be identified with the Kolmogorov length scale. This results in a Kolmogorov constant of $C=(2 / 3) \times(2 \pi)^{-4 / 3} \approx 0.0575$. This is almost two orders of magnitude smaller than the experimental value of $C \approx$ 1.5. Secondly, if we identify $\eta_{K}=1 / k_{\max }$ (which means that the inertial subrange of the system lies between $2 \pi \eta_{K}$ and $2 \pi / k_{\min }$, as suggested by Mariani et al., 2007), the resulting Kolmogorov constant is $C=2 / 3$. As a third approach, we can fix the Kolmogorov constant to be $C=3 / 2$ with the result that the Kolmogorov length scale is $\eta_{K}=(2 / 3)^{3 / 2} / k_{\max } \approx 0.544 / k_{\max }$ and thus $\eta_{K}$ is one order of magnitude smaller than the smallest wavelength simulated. We use this third solution in the following keeping in mind that the calculation of Reynolds numbers in kinematic simulations is not straightforward.

The Taylor-based Reynolds number can be derived from the definition in Eq. (2.51) using the turbulent kinetic energy in Eq. (3.22) with Eq. (3.23) and the kinematic 
viscosity from Eq. (3.26):

$$
\begin{aligned}
\mathcal{R}_{\lambda} & =\sqrt{\frac{20}{3}} \frac{k}{\sqrt{\nu \bar{\epsilon}}} \\
& =\sqrt{\frac{20}{3}} \frac{C \frac{3}{2} \bar{\epsilon}^{2 / 3}\left(1-\left(\frac{k_{\min }}{k_{\max }}\right)^{4 / 3}\right)^{-1}\left(k_{\min }^{-2 / 3}-k_{\max }^{-2 / 3}\right)}{\eta_{K}^{2 / 3} \bar{\epsilon}^{2 / 3}} \\
& =C \sqrt{15} \frac{1}{\eta_{K}^{2 / 3} k_{\max }^{2 / 3}} \frac{1}{x^{2 / 3}} \frac{1-x^{2 / 3}}{1-x^{4 / 3}} \quad \text { with } x=\frac{k_{\min }}{k_{\max }} .
\end{aligned}
$$

The Taylor-based Reynolds number with $C=3 / 2$ and $\eta_{K}=(2 / 3)^{3 / 2} / k_{\max }$ results in

$$
\mathcal{R}_{\mathrm{A}}=\left(\frac{3}{2}\right)^{2} \sqrt{15} \frac{1}{x^{2 / 3}} \frac{1-x^{2 / 3}}{1-x^{4 / 3}} \quad \text { with } x=\frac{k_{\min }}{k_{\max }} .
$$

In the literature, however, the Reynolds number in a kinematic simulation is sometimes defined as $\mathcal{R}_{\text {kinematic }} \equiv\left(k_{\max } / k_{\min }\right)^{4 / 3}$ (Nicolleau et al., 2016) which is not directly comparable with Reynolds numbers in DNSs or experiments.

\section{Characterization of the flow field}

An example of the Kraichnan flow field for $N_{F}=64$ modes is given in Fig. 3.3. A two-dimensional cut through the flow field shows differently sized structures which to the eye resemble a turbulent field. The probability distribution of the components of velocity and vorticity approach a Gaussian distribution for high numbers of Fourier modes. This is in agreement with the statement by Thomson and Devenish (2005) who proved that the velocity statistics (for large enough $N_{F}$ ) is always Gaussian. The variance of the velocity component is given by

$$
\begin{aligned}
\sigma_{u_{x}}^{2} & \equiv\left\langle u_{x}^{2}\right\rangle=\frac{1}{3}\left\langle\vec{u}_{\text {turb }}^{2}\right\rangle \\
& =\frac{2}{3} \int_{0}^{\infty} E(k) \mathrm{d} k \\
& =E_{0}\left(k_{\min }^{-2 / 3}-k_{\max }^{-2 / 3}\right)
\end{aligned}
$$




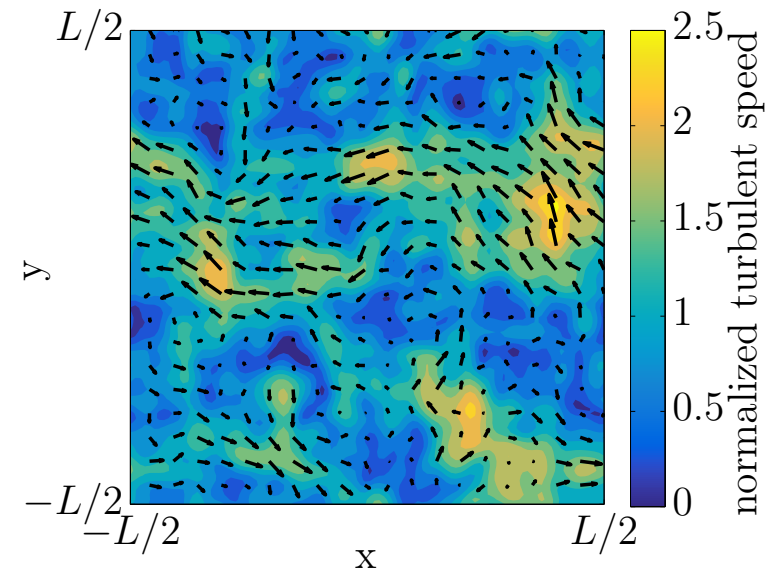

(a) Magnitude (color) and direction (small arrows) of the turbulent field with $N_{F}=64$ modes in a cross-section of the simulation box.
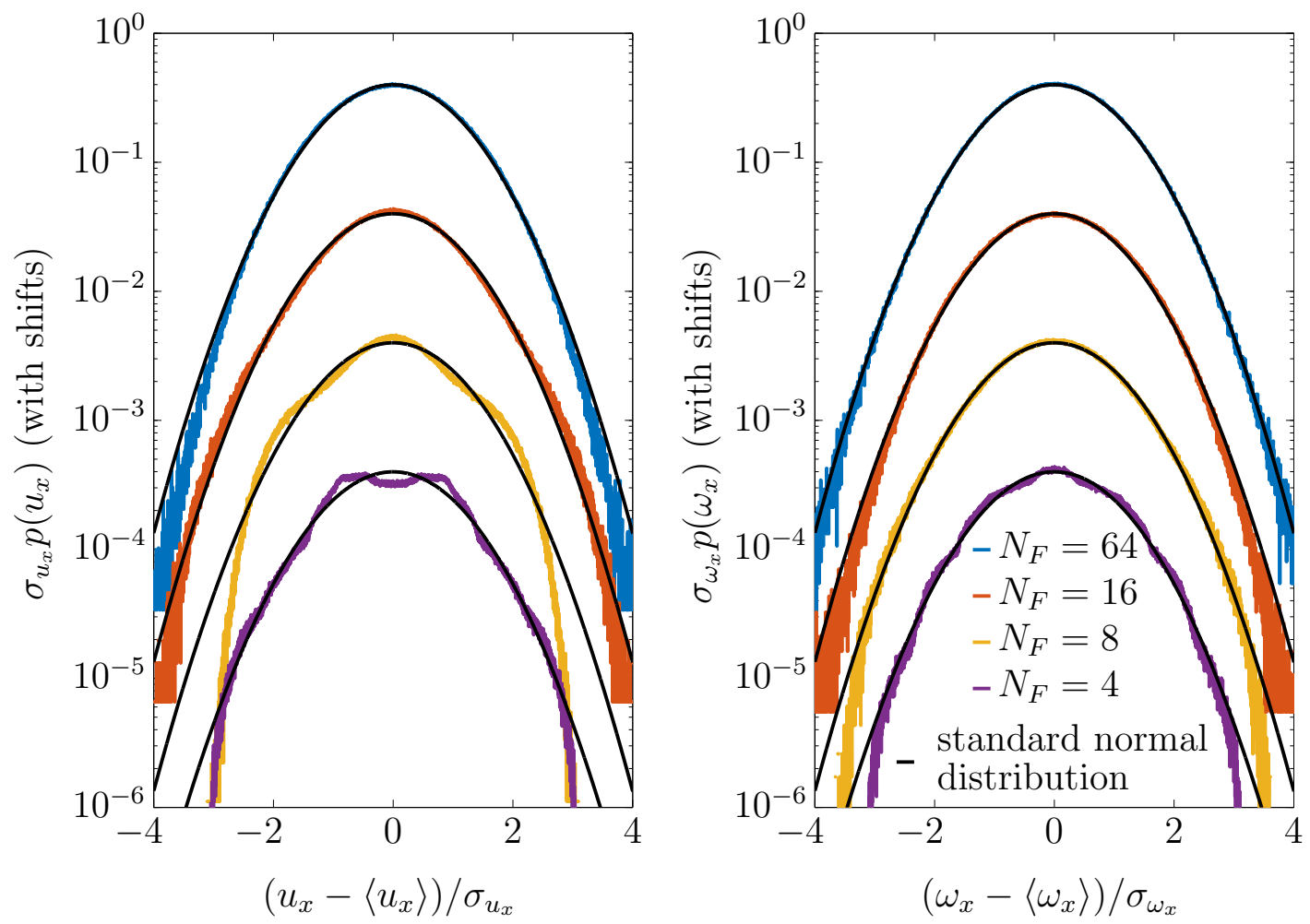

(b) Probability distributions of one component of the velocity and of one component of the vorticity for different numbers of Fourier modes. The curves are shifted for better visibility. The black lines denotes the standard normal distribution $p(x)=1 / \sqrt{2 \pi} \exp \left(-x^{2} / 2\right)$.

Figure 3.3: Example and probability distributions of the Kraichnan flow field. 


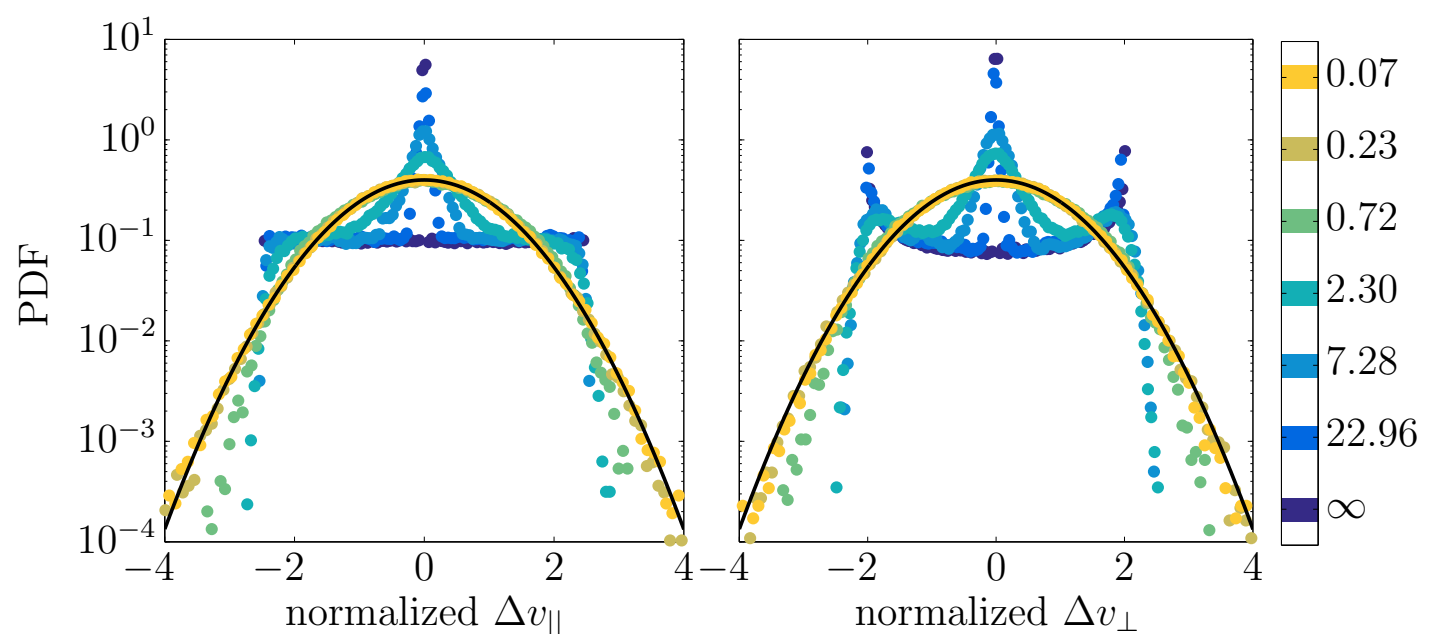

Figure 3.4: Eulerian velocity increments of the self-propelled particles in the Kraichnan flow field $\left(v_{0}=0.5\right)$. Different colors indicate different vortical Stokes numbers $\mathcal{S}_{\omega}=2 \gamma / \omega_{K}$. The zero mean, unit variance Gaussian distribution is plotted in black.

where Eqns. (2.25) and (3.18) have been used. Similarly, the variance of the vorticity can be derived as

$$
\begin{aligned}
\sigma_{\omega_{x}}^{2} & \equiv\left\langle\omega_{x}^{2}\right\rangle=\frac{1}{3}\left\langle\vec{\omega}^{2}\right\rangle \\
& =\frac{2}{3} \int_{0}^{\infty} k^{2} E(k) \mathrm{d} k \\
& =\frac{1}{2} E_{0}\left(k_{\max }^{4 / 3}-k_{\min }^{4 / 3}\right)
\end{aligned}
$$

where Eq. (2.27) together with Eqns. (2.26) and (3.18) have been used.

Phenomenological arguments suggest that the squared separation of two particles in a turbulent flow over time follows a third-order power law ("Richardson dispersion" Richardson, 1926; Monin and Yaglom, 1975, p. 574). However, for the Kraichnan method a sixth-order growth is found in the bulk and the pair separation follows a (classical) third-order power law in regions with very small velocities. This leads to an effective exponent of 9/2 (Thomson and Devenish, 2005).

\section{Velocity increments of particles in the Kraichnan flow field}

One important tool of analysis in the field of turbulence are velocity increments. These are used to characterize a flow field and compare it to a turbulent flow field. Two different sets of velocity increments can be calculated: Lagrangian and Eulerian. In the case of Lagrangian velocity increments we compare the velocities and positions of one single particle at two different time instants (separated by $\Delta t$ ). In contrary, to 
obtain the Eulerian velocity increments we compare velocities at positions separated by a distance $\Delta x$ at one and the same time. We will focus on Eulerian statistics in the following (cf. Wensink et al., 2012). In both cases the velocity difference $\overrightarrow{\Delta v}$ and the vector $\overrightarrow{\Delta x}$ connecting the two positions are used to calculate the longitudinal velocity increment

$$
\Delta v_{\|} \equiv \overrightarrow{\Delta v} \cdot \frac{\overrightarrow{\Delta x}}{\Delta x}
$$

The transverse velocity increment follows as

$$
\Delta v_{\perp} \equiv \overrightarrow{\Delta v} \cdot \hat{T}
$$

where $\hat{T}=\left(\epsilon_{i j} \hat{\Delta x}\right)$ is a unit vector perpendicular to the shift vector $\overrightarrow{\Delta x}$ (Wensink et al., 2012). We analyze the Eulerian velocity increments of self-propelled, point-like particles in a Kraichnan flow field $\left(N=27000, N_{F}=64, \mathcal{P}=\gamma / D_{r}=2 \times 10^{6}\right)$. Figure 3.4 shows examples of both the longitudinal as well as the transverse Eulerian velocity increments for different vortical Stokes numbers. The latter compares the strength of the nematic alignment mechanism with the turbulent vorticity (see Section 3.3.1). The curves of the velocity increments reveal a transition from Gaussian statistics (for low $\mathcal{S}_{\omega}$ ) when the turbulent flow dominates the movement of the particles, to a single peak at zero (for infinite $\mathcal{S}_{\omega}$ ) when the turbulent flow field vanishes. The transverse velocity increments shows in the latter case also two peaks at \pm 2 which is a purely geometric effect and can be calculated analytically (Gillespie, 1983). We can conclude that the Kraichnan flow field shows Gaussian statistics and that there is a smooth transition from the turbulence dominated system to the purely self-propelled system.

\subsubsection{Direct numerical simulations}

Apart from the Kraichnan model, we also use the flow field generated by a direct numerical simulation ${ }^{1}$. The DNS uses a standard pseudo-spectral method (details can be found in Wilczek, 2010) to solve the incompressible Navier-Stokes equations

$$
\begin{aligned}
\partial_{t} \vec{u}_{\text {turb }}(\vec{r}, t)+\vec{u}_{\text {turb }}(\vec{r}, t) \cdot \nabla \vec{u}_{\text {turb }}(\vec{r}, t) & =-\nabla p+\nu \Delta \vec{u}_{\text {turb }}(\vec{r}, t)+\vec{f}(\vec{r}, t), \\
\nabla \cdot \vec{u}_{\text {turb }}(\vec{r}, t) & =0 .
\end{aligned}
$$

\footnotetext{
${ }^{1}$ The numerical data is kindly provided by Cristian C. Lalescu from the group "Theory of Turbulent flows" (head: Michael Wilczek) at the Max Planck Institute for Dynamics and Self-Organization, Göttingen.
} 


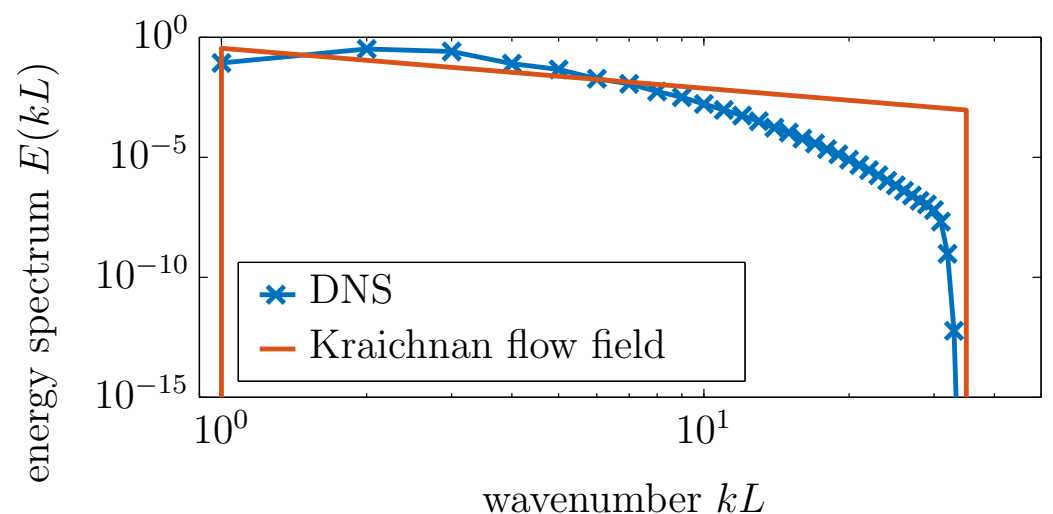

Figure 3.5: Resulting energy spectrum of the direct numerical simulation (blue) and prescribed Kolmogorov spectrum of the Kraichnan flow field (red).

The forcing $\vec{f}$ is of the Lundgren type (Lundgren, 2003; Rosales and Meneveau, 2005) with

$$
\hat{f}(\vec{k}, t)= \begin{cases}\alpha \hat{u}_{\text {turb }}(\vec{k}, t) & \text { for } k_{0}^{f} \leq k \leq k_{1}^{f} \\ 0 & \text { otherwise }\end{cases}
$$

The real space grid consists of $72 \times 72 \times 72$ nodes at a Taylor-based Reynolds number of $\mathcal{R}_{\lambda} \approx 17$. A high spatial resolution of $\max (k) \cdot \eta_{K} \approx 2.2$ (with the Kolmogorov scale $\left.\eta_{K}\right)$ can be achieved. We follow Ishihara et al. (2007) for the calculation of standard statistical quantities. The resulting energy spectrum (Fig. 3.5) clearly differs from the Kolmogorov spectrum and does not show a power-law decay. This is one of the main differences between the DNS and the Kraichnan method. A second discrepancy concerns the statistics which is Gaussian for the Kraichnan flow field and can deviate from Gaussianity in the case of DNSs.

\subsection{Molecular dynamics simulations}

The structure of the MD simulation code is given in Fig. 3.6. The centerpiece of the code is the time loop which includes the integration of the equations of motion and the application of boundary conditions. The input parameters are given in Tab. 3.1.

The equations of motion are integrated by using the Euler-Maruyama scheme (Maruyama, 1955). ${ }^{2}$ A time step $\Delta t$ is introduced and positions and orientations

\footnotetext{
${ }^{2}$ Higher-order integration schemes could be used but are not expected to have a significant impact on the results due to the stochastic nature of the equations.
} 


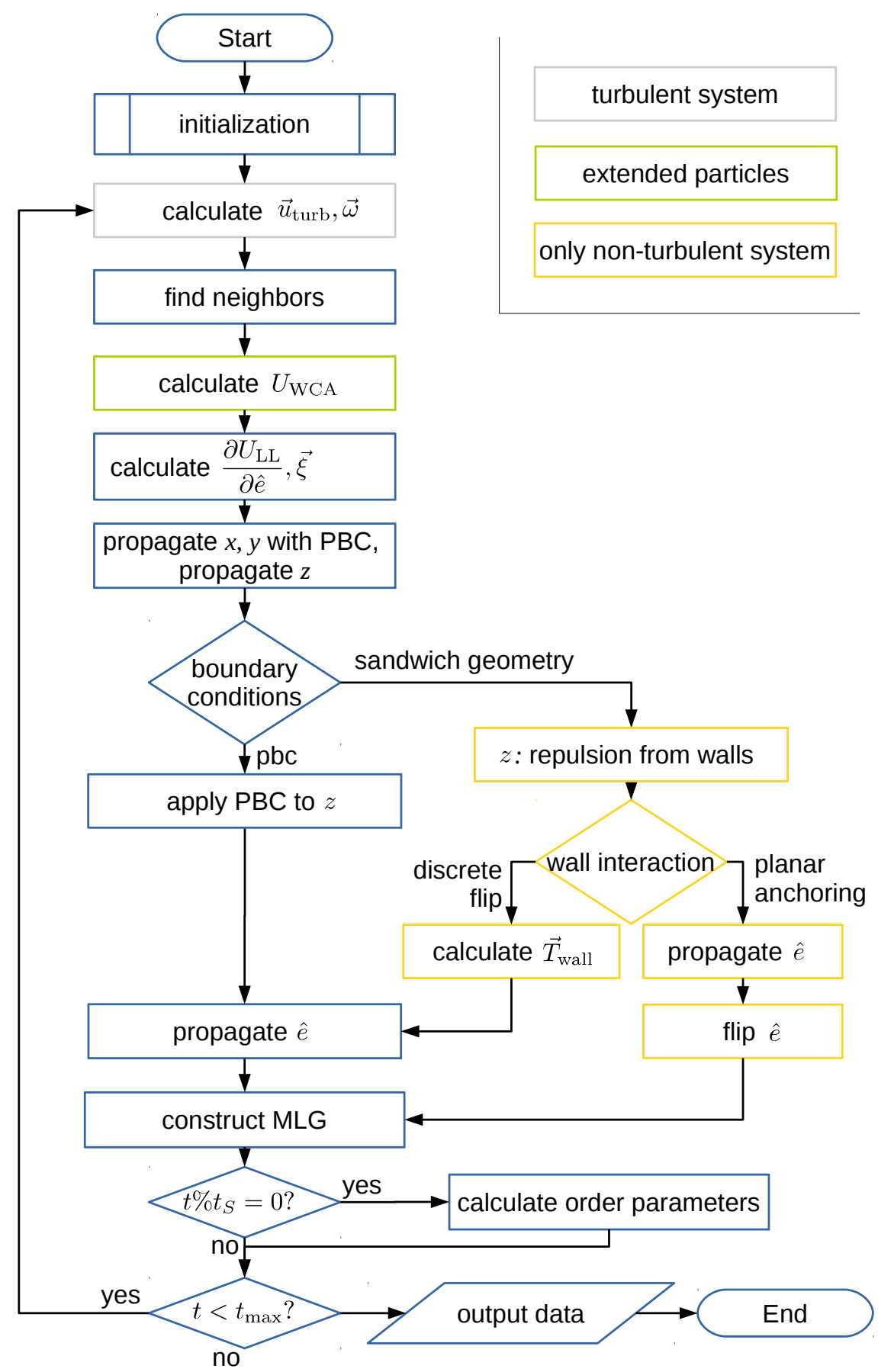

Figure 3.6: Schematic structure of the MD program to model nematically aligning active swimmers (extended or point-like) with and without a surrounding flow field. The "initialization" includes the input of parameters (see Tabs. 3.1 and 6.1) and the initialization of the particle positions and orientations, as well as the Kraichnan flow field. The percentage sign $\left(t \% t_{S}\right)$ refers to the modulo operation and thus the order parameters are calculated in time intervals of $t_{S}$. 


$\left.\begin{array}{ll}\hline \text { Parameter } & \text { symbol } \\ \hline \text { number of particles } & N_{x} N_{y} N_{z} \\ \text { side lengths of simulation box } & L_{x} L_{y} L_{z} \\ \text { nematic interaction range } & \epsilon \\ \text { self-propulsion speed } & v_{0} \\ \text { strength of noise } & \eta \\ \text { nematic relaxation constant } & \gamma \\ \text { time step } & \Delta t \\ \text { number of iterations } & N_{\text {iter }}\end{array}\right\}$ general

Table 3.1: Input parameters for the simulations.

are advanced according to

$$
\begin{aligned}
& \vec{r}_{i}(t+\Delta t)=\vec{r}_{i}(t)+\vec{v}_{i} \Delta t \\
& \hat{e}_{i}(t+\Delta t)=\hat{e}_{i}(t)+\left[\vec{T}_{i}\right]_{\perp} \Delta t+\lambda \hat{e}_{i}(t) \Delta t
\end{aligned}
$$

with velocity $\vec{v}_{i}$ and torque $\vec{T}_{i}$. The integration scheme is the same for any of the four models with the corresponding velocity $\vec{v}_{i}$ and torque $\left[\vec{T}_{i}\right]_{\perp}$ : Point particles (Eq. 3.2), extended particles (Eq. 3.5), point particles in a turbulent field (Eq. 3.7), or extended particles in a turbulent field (Eq. 3.8). The subscript $\perp$ refers to the part of the vector which is perpendicular to $\hat{e}_{i}$ as defined in Eq. (3.3). $\lambda$ is a Lagrangian multiplier which keeps the length of the orientation vector fixed (Ilnytskyi and Wilson, 2002) and which can be derived from the conditions $\hat{e}_{i}(t)^{2}=\hat{e}_{i}(t+\Delta t)^{2}=1$ and $\hat{e}_{i}(t) \cdot \hat{e}_{i}(t+\Delta t)>0$ as

$$
\lambda \Delta t=-1+\sqrt{1-\left(\vec{T}_{i, \perp} \Delta t\right)^{2}} .
$$

The use of the Lagrangian multiplier hence constrains the time step to $\Delta t<\sqrt{1 /\left|\vec{T}_{\perp}\right|}$. The particles' positions inside the simulation domain are within the ranges $-L_{\alpha} / 2 \leq$ $\alpha \leq L_{\alpha} / 2$ for $\alpha \in[x, y, z]$. Internally all positions are saved as $\alpha_{\text {internal }}=\alpha / L_{\alpha}$. This approach is computationally more efficient because the periodic boundary conditions can be applied very easily. 


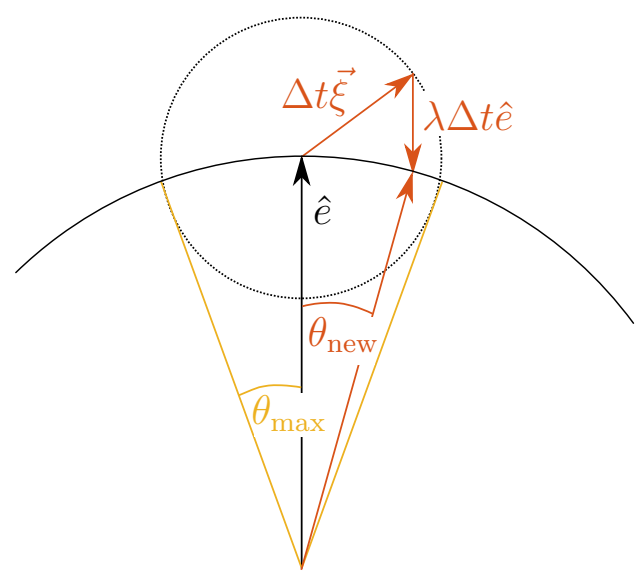

(a) Sketch of our implementation of the rotational noise. To the original orientation $\hat{e}$ a random vector on the surface of a sphere $\eta \Delta t \hat{\xi}$ is added and the correction by the Lagrangian multiplier $\lambda \Delta t \hat{e}$ is applied.

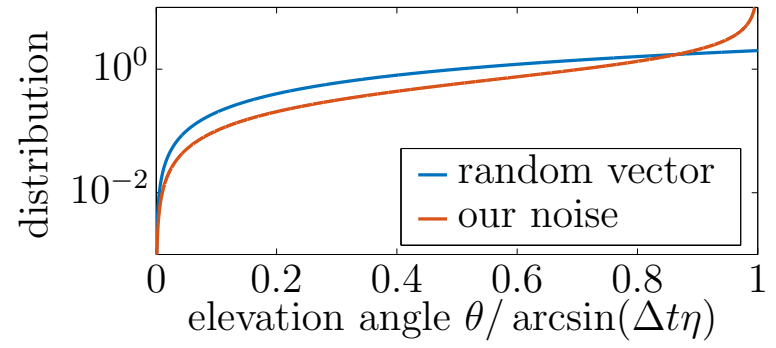

(b) Analytically derived (normalized) angular probability distributions for our noise (Eq. 3.45, red curve) and for a random vector in a spherical sector (Eq. 3.47, blue curve).

Figure 3.7: Implementation of rotational stochastic noise in our model.

\subsubsection{Rotational noise}

The stochastic noise is implemented as follows: For each particle at every time step a vector $\vec{\xi}_{i}(t)$ is generated, which is distributed randomly on a sphere (see Allen and Tildesley, 1989) of radius $\eta$ (Czirók et al., 1999). For the propagation of the orientation $\hat{e}$ (in the absence of particle-particle interactions) the random vector $\Delta t \vec{\xi}$ is added to the orientation vector plus a correction $\lambda \Delta t \hat{e}$ with the Lagrangian multiplier $\lambda$ to keep the length of $\hat{e}$ fixed (see Fig. 3.7a). The resulting possible new orientations form a spherical sector with the half cone angle $\theta_{\max }$ which is a function of $\Delta t$ and $\eta$. In the following we will derive the angular probability distribution of this new orientation. We choose our coordinate system such that $\hat{e}=\hat{z}$. The new orientation, after stochastic noise has been applied, is then given by

$$
\begin{aligned}
\hat{e}_{\text {new }} & =\hat{e}+\Delta t \vec{\xi}_{\perp}+\lambda \Delta t \hat{e} \\
& =\Delta t \vec{\xi}_{\perp}+\sqrt{1-\left(\Delta t \vec{\xi}_{\perp}\right)^{2}} \hat{e}
\end{aligned}
$$


where we used Eq. (3.36) for the Lagrangian multiplier. The random vector $\vec{\xi}$ is given by

$$
\vec{\xi}=\eta\left(\begin{array}{c}
\sin \theta \cos \phi \\
\sin \theta \sin \phi \\
\cos \theta
\end{array}\right)
$$

with equally distributed $\phi \in[0,2 \pi)$ (probability distribution $p(\phi)=(2 \pi)^{-1}$ ). The elevation angle $\theta$ can be constructed as

$$
\theta=\arccos (2 \alpha-1)
$$

with equally distributed $\alpha \in[0,1)$ (and hence $p(\alpha)=1$ ) to achieve a random distribution on the surface of a sphere. This originates from the surface element of the sphere which is given by

$$
\mathrm{d} \Omega=\sin \theta \mathrm{d} \theta \mathrm{d} \phi=-\mathrm{d}(\cos \theta) \mathrm{d} \phi .
$$

With these new coordinates the new orientation can then be written as

$$
\hat{e}_{\text {new }}=\left(\begin{array}{c}
\eta \Delta t \sin \theta \cos \phi \\
\eta \Delta t \sin \theta \sin \phi \\
\sqrt{1-(\eta \Delta t \sin \theta)^{2}} .
\end{array}\right)
$$

The azimuthal angle

$$
\phi_{\text {new }}=\arctan \left(\frac{\hat{e}_{\text {new }} \cdot \hat{y}}{\hat{e}_{\text {new }} \cdot \hat{x}}\right)=\phi
$$

is equal to the azimuthal angle of the random vector $\vec{\xi}$ and $\left|\hat{e}_{\text {new }}\right|^{2}=1$. We are now interested in deriving the probability distribution of the elevation angle $\theta_{\text {new }}$. We can express the elevation angle of the random vector $\vec{\xi}$ as

$$
\sin \theta=\sin (\arccos (2 \alpha-1))=2 \sqrt{\alpha(1-\alpha)}
$$

and hence the elevation angle of the orientation is given by

$$
\begin{aligned}
\theta_{\text {new }} & =\arccos \left(\frac{\hat{e}_{\text {new }} \cdot \hat{z}}{\left|\hat{e}_{\text {new }}\right|}\right) \\
& =\arccos \sqrt{1-(\eta \Delta t \sin \theta)^{2}} \\
& =\arccos \sqrt{1-(2 \eta \Delta t)^{2} \alpha(1-\alpha)} .
\end{aligned}
$$

It vanishes for $\alpha=0$ and $\alpha=1$, and assumes it maximum value $\theta_{\max }=\arcsin (\eta \Delta t)$ at $x=1 / 2$. We can derive the probability distribution of $\theta_{\text {new }}$ following Gillespie 
(1983) and using the probability distribution of $\alpha$ :

$$
p\left(\theta_{\text {new }}\right)=\int p(x) \delta\left(\theta_{\text {new }}-\arccos \sqrt{1-c(2 \eta \Delta t)^{2} \alpha(1-\alpha)}\right) \mathrm{d} \alpha .
$$

We substitute $a \equiv \arccos \sqrt{1-c(2 \eta \Delta t)^{2} \alpha(1-\alpha)}$ which leads to the probability distribution (not normalized)

$$
\begin{aligned}
p\left(\theta_{\text {new }}\right) & =\int \frac{\sqrt{\alpha(1-\alpha)-[2 \eta \Delta t \alpha(1-\alpha)]^{2}}}{\eta \Delta t(1-2 \alpha)} \delta\left(\theta_{\text {new }}-a\right) \mathrm{d} a \\
& =\int \frac{\sqrt{\left(1-\cos ^{2} a\right) /(2 \eta \Delta t)^{2}-\left(1-\cos ^{2} a\right) /(2 \eta \Delta t)^{2}}}{\eta \Delta t\left((2 \eta \Delta t)^{-1} \sqrt{2 \cos (2 a)+(2 \eta \Delta t)^{2}-2}\right.} \delta\left(\theta_{\text {new }}-a\right) \mathrm{d} a \\
& =\frac{1}{\eta \Delta t} \sqrt{\frac{\cos ^{2} \theta_{\text {new }}-\cos ^{4} \theta_{\text {new }}}{2 \cos \left(2 \theta_{\text {new }}\right)+(2 \eta \Delta t)^{2}-2}} .
\end{aligned}
$$

This distribution (see red curve in Fig. 3.7b) diverges as $\theta_{\text {new }}=\theta_{\max }=\arcsin (\eta \Delta t)$.

As a comparison, we also derive the angular probability distribution of a random vector $\theta_{\text {rand }}$ in a spherical segment. The azimuthal angle $\phi_{\text {rand }} \in[0,2 \pi)$ is again equally distributed. The elevation angle follows directly

$$
\theta_{\text {rand }}=\arccos (2 \alpha-1)
$$

with equally distributed $\alpha \in[0,1)$. The resulting angular distribution is

$$
\begin{aligned}
p\left(\theta_{\text {rand }}\right) & =\int p(\alpha) \delta\left(\theta_{\text {rand }}-\arccos (2 \alpha-1)\right) \mathrm{d} x \\
& =\int \sqrt{\alpha(1-\alpha)} \delta\left(\theta_{\text {rand }}-\arccos (2 \alpha-1)\right) \mathrm{d} a \\
& =\frac{1}{2} \sqrt{\left(\cos \theta_{\text {rand }}+1\right)\left(1-\cos \theta_{\text {rand }}\right)}
\end{aligned}
$$

where the substitution $a=\arccos (2 \alpha-1)$ has been used. We see that this angular distribution also increases with the angle $\theta$ (blue curve in Fig. 3.7b). However, our implementation of rotational noise leads to a higher probability for larger angles compared to the distribution of random vectors in a spherical segment.

The noise is delta-correlated in time and space: $\left\langle\xi_{i \alpha}(t) \times \xi_{j \beta}\left(t^{\prime}\right)\right\rangle=2 D_{r} \delta\left(t-t^{\prime}\right) \delta_{\alpha \beta}$ where $D_{r}$ is the rotational diffusion constant $D_{r}$. After discretization in time this leads to $\left\langle\xi_{i \alpha}(t) \times \xi_{j \beta}(t+\Delta t)\right\rangle=2 D_{r} \frac{1}{\Delta t} \delta_{\alpha \beta}$ from which the rotational diffusion constant follows as

$$
D_{r}=\frac{\eta^{2} \Delta t}{2}
$$



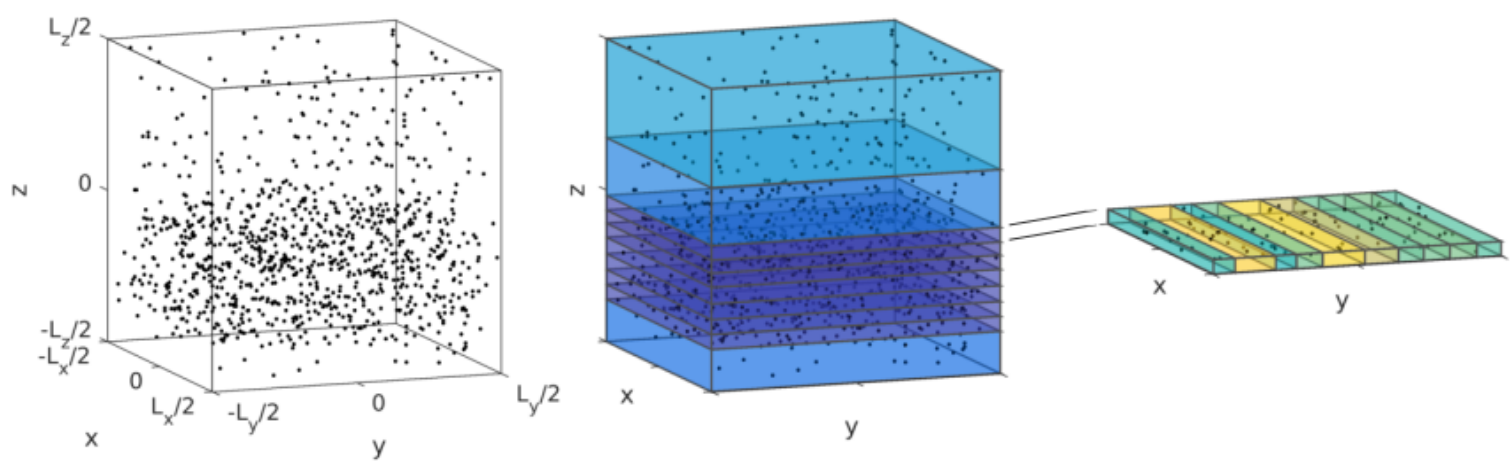

Figure 3.8: Illustration of the monotonic logical grid: The left panel shows all particles in the simulation box with a non-homogeneous density distribution. The middle panel shows the division of the simulation box into $N_{z}$ layers which are not of equal size. Finally, the division of one such layer into $N_{y}$ bars is shown in the right panel. The colors in the middle and right panels indicate the height of the layer and the width of the bar, respectively.

The system does not obey the fluctuation-dissipation theorem since there is no coupling between the stochastic and the deterministic part of the orientation equation.

\subsubsection{Monotonic logical grid}

The equations in this system are easy to integrate and not extremely computationally expensive. The most costly part is the neighbor search to find neighbors within the interaction range of a particle. Most MD simulations use some kind of neighbor-list algorithm to achieve this goal. However, we implemented (see Appendix A.4) the method of the monotonic logical grid (Boris, 1986; Weinketz, 1993). This method is an efficient search of neighboring particles based on the idea that particles which are close to each other in real space should also be close to each other in computational memory.

The simulation domain is divided into subboxes which are not equally distant but each contains the same number of particles (see Fig. 3.8): In a first step $N_{z}$ layers are defined each of which contains $N_{x} N_{y}$ particles. Each layer is then subdivided into $N_{y}$ bars consisting of $N_{x}=N /\left(N_{y} N_{z}\right)$ particles each. The particles are not only assigned to a specific layer and bar, but are also saved in memory accordingly such that particles which are close to each other in physical space are also stored in the vicinity of each other.

For each layer and each bar the maximum and minimum values of $z_{i}$ are known, as well as the maximum and minimum $y_{i}$ values for each bar. For a given particle $i$ the algorithm first finds the range of layers in which neighbors could potentially be by comparing the $z_{i}$ coordinate of the particle to the bounding values $z_{\min }$ and $z_{\max }$ 


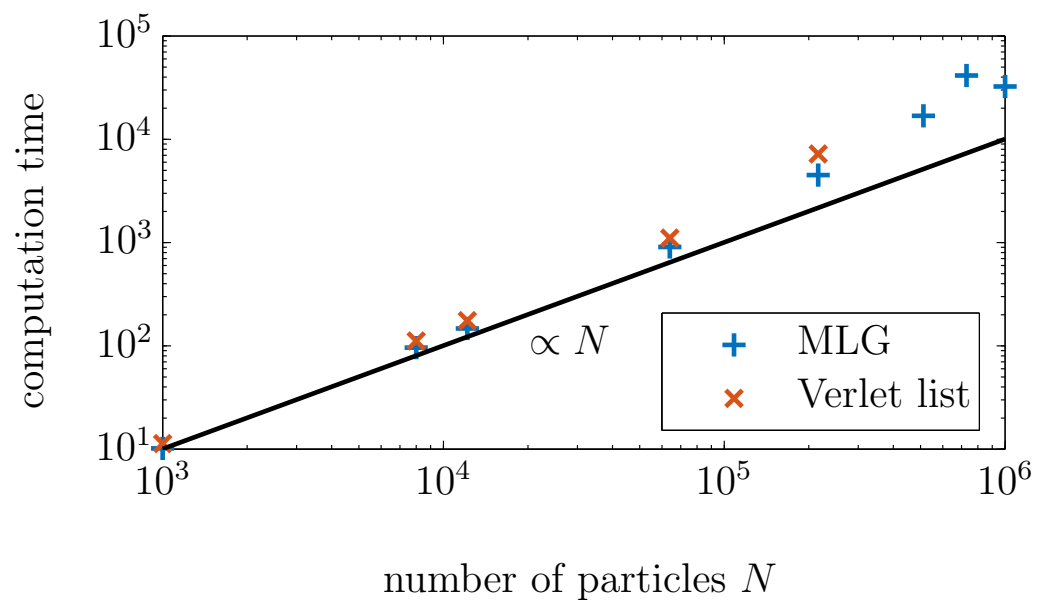

Figure 3.9: Run times of simulations with different numbers of particles using the MLG-algorithm or a Verlet neighborlist (symbols). The solid black line indicates linear behavior.

of the layers. In a second step, the bars which contain potential neighbors are found by comparing the values of the $y_{i}$ coordinate of the particles to the bounding values $y_{\min }$ and $y_{\max }$ of the bars within neighboring layers. And finally, the full positions of the individual particles inside these neighboring bars are compared to the position of particle $i$. All of these searches are done by keeping the overhead as small as possible, i.e. the search starts at the position of the particle and does not proceed once a layer, bar, or $x$ position of another particle outside the interaction range is found. Moreover, it is assured that each pair of particles is considered only once. After each integration step the monotonic logical grid is reconstructed, i.e. the particles are assigned to their specific layer and bar and saved in memory in this ordered fashion.

One drawback of this method is that the number of simulated particles $N$ must be decomposed as a product of three integers. It will be most efficient in a cubic box if the integers are of roughly the same size.

We compare the efficiency of this method to a standard Verlet neighborlist algorithm (Allen and Tildesley, 1989, p. 147). Figure 3.9 shows a linear dependency of the computation time as a function of the number of particles for both methods. The performance of the MLG code is, however, slightly better than the performance of the Verlet neighborlist.

\subsubsection{Particles in a turbulent field}

The implementation of the equations of motion of particles in a turbulent field (Eq. 3.7) is done completely analogously to the simple system. The main differ- 
ence is that the turbulent field has to be calculated. For the Kraichnan flow field, this is easily performed in a single step: we calculate the velocity (Eq. 3.9) and vorticity (Eq. 3.10) for every particle position. However, for the simulations which include results from DNS an approximation is necessary. The turbulent velocity and vorticity are saved during the DNS on a spatial grid and loaded into memory during the MD simulation. They are then interpolated at the particle sites via a trilinear interpolation scheme.

\subsubsection{Boundary conditions}

The MD simulations are carried out in a three-dimensional rectangular domain with side lengths $L_{x}, L_{y}, L_{z}$ and $|\alpha| \leq L_{\alpha} / 2$ for $\alpha \in(x, y, z)$. In the following, several possible boundary conditions and their implementations are discussed.

\section{Periodic boundary conditions}

The PBCs are probably the most common choice in MD simulations (Allen and Tildesley, 1989, p. 24). When a particle leaves the simulation box on one side, it reenters on the opposite side at the mirror position. The system is thought to be replicated in the three directions, similarly to a crystal which is composed of an (in principle) infinite number of repetitions of the primitive cell. We then pick one of these cells to carry out the simulations. The main advantage of PBCs is that the system does not have boundaries and hence no boundary effects will occur. However, one drawback is that some artifacts can arise like, for instance, when periodic structures are simulated only the commensurate wavelengths with the system size will emerge (see also the discussion in Section 4.3). Moreover, percolated (dense) structures are more likely to align along one axis of the box because they are self-connecting through PBCs (Ginelli et al., 2010).

PBCs are implemented by applying the following transformation to a particle coordinate $\alpha$ :

$$
\alpha_{\mathrm{PBC}}=\alpha-\text { round }\left(\frac{\alpha}{L_{\alpha}}\right) L_{\alpha}
$$

where the round function refers to rounding to the nearest integer.

\section{Sandwich geometry}

To study the influence of walls onto the system, it is useful to perform simulations in a slit-pore or sandwich geometry: Two parallel walls on the top and on the bottom of the simulation box and periodic boundary conditions in the other directions. The particle-wall interaction is commonly taken as repulsive, and modeled with a WCA potential (Weeks et al., 1971) in analogy to the inter-particle interaction of extended 

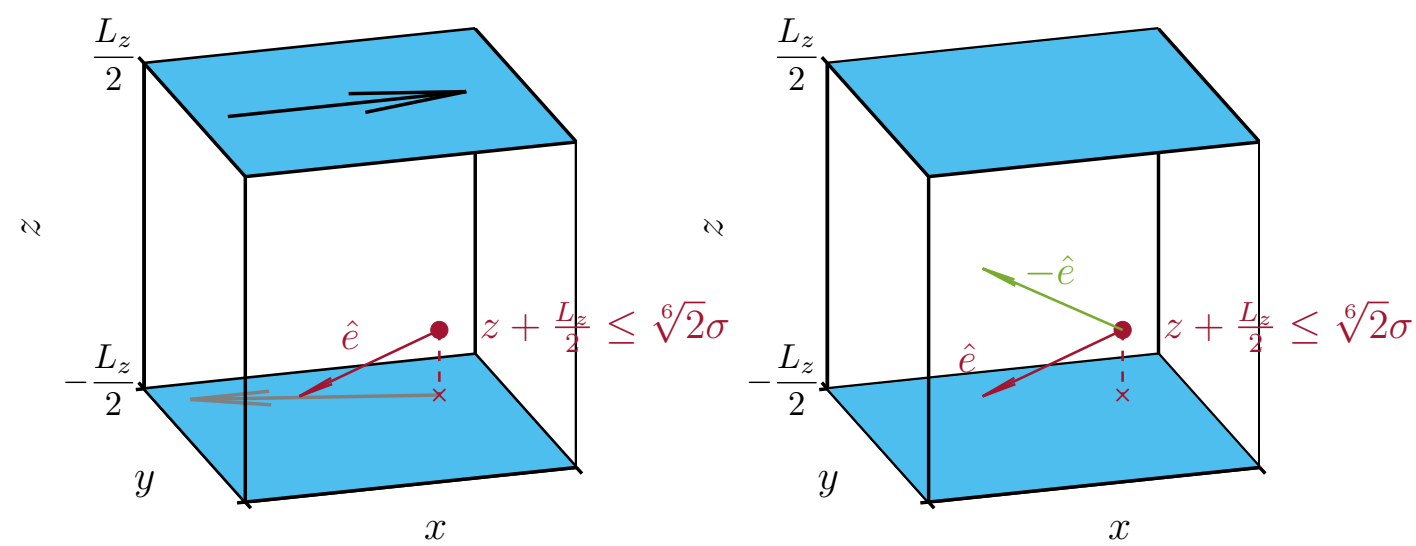

Figure 3.10: Sketch of different boundary conditions in the sandwich geometry. The walls are given in blue, periodic boundary conditions are applied in the other directions. The left panel describes planar anchoring while the right panel illustrates a discrete flip of the orientation close to the wall.

particles (see Section 3.1.2). The potential is given by

$$
U_{\text {wall }}(\Delta z)= \begin{cases}4 \epsilon_{1}\left[\left(\frac{\sigma}{\Delta z}\right)^{12}-\left(\frac{\sigma}{\Delta z}\right)^{6}\right]+\epsilon_{1} & \begin{array}{l}
\text { for } \Delta z=z-\frac{L_{z}}{2} \geq-\sqrt[6]{2} \sigma \\
\text { and for } \Delta z=z+\frac{L_{z}}{2} \leq \sqrt[6]{2} \sigma
\end{array} \\
0 & \text { otherwise }\end{cases}
$$

with the energy scale $\epsilon_{1}$ and the particle diameter $\sigma$. This potential alters the equation of motion for the $z$ component of the position. An additional force

$$
F_{z}=-\frac{1}{\gamma_{F}} \frac{\partial U_{\text {wall }}}{\Delta z}=\frac{24 \epsilon_{1}}{\gamma_{F}} \frac{1}{\Delta z}\left[2\left(\frac{\sigma}{\Delta z}\right)^{6}-1\right]\left(\frac{\sigma}{\Delta z}\right)^{6}
$$

is added to the equation for $\dot{z}$ with the relaxation time $\gamma_{F}$. Here, $\sigma$ is not the particle diameter but rather the thickness of the wall.

If we do not assume any influence of the walls onto the orientation of the particles, the particles would get stuck at the walls due to the persistent speed once they approach the wall. There is no deceleration of the particles through the particle-wall interaction and so the particles would continue in trying to penetrate the wall. We therefore need the walls to change also the orientation of an approaching particle. Two different models to achieve that are presented in the following.

The first model is motivated by the similarity of our SPPs with liquid crystals. To rod-like liquid crystals usually two different types of preferential alignment at interfaces (anchoring) are applied both numerically and experimentally: either homeotropic anchoring or planar anchoring. The homeotropic anchoring is not useful in our case 
because it favors the particle orientation perpendicular to the wall which does not solve the described problem. So we will use a boundary condition similar to planar anchoring (see Fig. 3.10, left panel). The nematic alignment of a particle with a vector $\hat{e}_{\text {wall }}$ can be described by the Lebwohl-Lasher potential (cf. Eq. 3.1)

$$
U_{\text {wall }}^{\text {planar }}= \begin{cases}-\left(\hat{e} \cdot \hat{e}_{\mathrm{wall}}\right)^{2} & , \text { for } z-\frac{L_{z}}{2} \geq-\sqrt[6]{2} \sigma \text { or } z+\frac{L_{z}}{2} \leq \sqrt[6]{2} \sigma \\ 0 & , \text { otherwise }\end{cases}
$$

The resulting torque $-\gamma_{w} \partial U_{\text {wall }}^{\text {planar }} / \partial \hat{e}$ is added to the equation of motion of $\hat{e}$ with the relaxation constant $\gamma_{w}$. The alignment vector is chosen as

$$
\hat{e}_{\mathrm{wall}}= \begin{cases}\hat{x} & , \text { for the upper wall } \\ \frac{1}{|\hat{e}-(\hat{e} \cdot \hat{z}) \hat{z}|}(\hat{e}-(\hat{e} \cdot \hat{z}) \hat{z}) & , \text { for the lower wall }\end{cases}
$$

so that the particles tend to align parallel to the $x$-axis close to the upper wall. The alignment at the lower wall is parallel to the wall but not in a fixed direction which allows the system to self-organize and find its preferred direction.

We turn now to the second model which can be applied to model the interaction between SPPs and hard walls. The idea is to mimic an elastic reflection of the particles. To this end we apply a discrete flip to the particle orientation if it is positioned close to the wall and its orientation is pointing toward the wall:

$$
\hat{e}_{\text {new }}=-\hat{e}\left\{\begin{array}{l}
\text { for } z-L_{z} / 2 \geq-\sigma \text { and } \hat{e} \cdot \hat{z}>0 \\
\text { and for } z+L_{z} / 2 \leq \sigma \text { and } \hat{e} \cdot \hat{z}<0
\end{array}\right.
$$

The disadvantage of this model is that it is discontinuous in time. The influence of such a sandwich geometry onto the dynamics of the system and the differences between the two different models are discussed in Section 4.5.

\subsection{Description of the system}

In the Section we introduce several parameters to describe the system: dimensionless measures which we control for every simulation, and order parameters which we calculate in the steady state. Moreover, we describe different ways to measure the degree of clustering in our system. 


\subsubsection{Dimensionless measures of self-propelled particles and flow}

\section{Péclet number}

The relative importance of nematic alignment relaxation rate with respect to the rotational diffusivity related to the stochastic noise is described by a rotational Péclet number. In general, the Péclet number is defined as the ratio of advective over diffusive fluxes. When interested only in the orientational part of the equations of motion (Eq. 3.2), we can identify two influences: The stochastic noise leads to rotational diffusion with the diffusion constant $D_{r}=\eta^{2} \Delta t / 2$ (see Section 3.2). On the other hand, we have nematic alignment of the particles with its neighbors which is described by its strength $\gamma$. This alignment can be thought of as a "rotational advection" because it is the influence of the surrounding (neighboring particles) onto a particle itself. The rotational Péclet number (see Guasto et al., 2012, for a similar definition) is hence given by

$$
\mathcal{P} \equiv \frac{\text { nematic alignment }}{\text { rotational diffusion }}=\frac{\gamma}{D_{r}}=\frac{2 \gamma}{\eta^{2} \Delta t} .
$$

\section{Stokes number}

For the system of SPPs in a turbulent field we solve Eq. (3.7). The orientational equation involves three terms, so we use two dimensionless numbers to describe the system, each defined as the ratio between two terms. The first dimensionless number is the Péclet number as before (Eq. 3.55): It compares the strength of nematic alignment to the stochastic noise. The second dimensionless number weighs the influence of the turbulent field (through the vorticity) against the stochastic noise. We compare the characteristic times of nematic alignment $\tau_{\text {nem }} \sim \gamma^{-1}$ to the characteristic time of turbulent vorticity $\tau_{\text {turb }} \sim 2 \omega_{K}^{-1}$. The resulting dimensionless number is called "vortical Stokes number":

$$
\mathcal{S}_{\omega} \equiv \frac{\text { nematic alignment }}{\text { turbulent vorticity }}=\frac{\tau_{\text {turb }}}{\tau_{\text {nem }}}=\frac{2 \gamma}{\omega_{K}} .
$$

We can construct a third dimensionless number as the ratio of $\mathcal{P}$ to $\mathcal{S}_{\omega}$.

\section{Stokes number with speed correction}

The vortical Stokes number is not enough to describe the onset of global nematic order as a function of the strength of the turbulent field in comparison to the strength of the nematic alignment. In fact, we need to take into account the self-propulsion speed 
of the particle as well as the turbulent speed advecting them. For negligible noise $(\mathcal{P} \rightarrow \infty)$, a (point-)particle in a time step $\Delta t$

1. travels a distance $\Delta s_{\mathrm{SPP}}=v_{0} \Delta t$,

2. is advected a distance $\Delta s_{\text {turb }}=u_{\mathrm{rms}} \Delta t$,

3. alignes nematically with an angle $\Delta \phi_{\text {nem }}=\gamma \Delta t$, and

4. is rotated by the turbulent field at an angle $\Delta \phi_{\text {turb }}=\frac{1}{2} \omega_{K} \Delta t$.

The plane angles $\Delta \phi$ describe cones of solid angles

$$
\Omega=4 \pi\left(\sin \frac{\Delta \phi}{4}\right)^{2}
$$

around the orientiation $\hat{e}$ of the particle. Since the angles $\Delta \phi$ are supposed to be small ( $\Delta t$ has to be chosen small enough, see derivation of the Lagrangian multiplier in Section 3.2), we can make use of the small-angle approximation $\sin x \approx x$. We can now compare two different areas (in the sense of cross sections): Firstly, there is the spherical cap that is given by $\Delta s_{\text {SPP }}$ and $\Delta \phi_{\text {nem }}$ which is a measure of the tendency of the system towards global nematic alignment:

$$
\begin{aligned}
A_{\mathrm{SPP}} & =\Omega_{\mathrm{nem}}\left(\Delta s_{\mathrm{SPP}}\right)^{2}=4 \pi\left(\sin \frac{\Delta \phi_{\mathrm{nem}}}{4}\right)^{2}\left(\Delta s_{\mathrm{SPP}}\right)^{2} \\
& \approx 4 \pi\left(\frac{\Delta \phi_{\mathrm{nem}}}{4}\right)^{2}\left(\Delta s_{\mathrm{SPP}}\right)^{2}=\frac{\pi}{4}\left(\gamma v_{0}(\Delta t)^{2}\right)^{2} .
\end{aligned}
$$

The second cross section describes the effect of the turbulent field to destroy nematic order:

$$
\begin{aligned}
A_{\text {turb }} & =\Omega_{\text {turb }}\left(\Delta s_{\text {turb }}\right)^{2}=4 \pi\left(\sin \frac{\Delta \phi_{\text {turb }}}{4}\right)^{2}\left(\Delta s_{\text {turb }}\right)^{2} \\
& \approx 4 \pi\left(\frac{\Delta \phi_{\text {turb }}}{4}\right)^{2}\left(\Delta s_{\text {turb }}\right)^{2}=\frac{\pi}{4}\left(\frac{1}{2} \omega_{K} u_{\mathrm{rms}}(\Delta t)^{2}\right)^{2} .
\end{aligned}
$$


For nematic order to occur, $A_{\mathrm{SPP}}$ has to be larger than $A_{\text {turb }}$ :

$$
\begin{array}{rlrl}
A_{\mathrm{SPP}} & >A_{\mathrm{turb}} \\
\Rightarrow \quad \frac{\pi}{4}\left(\gamma v_{0}(\Delta t)^{2}\right)^{2} & >\frac{\pi}{4}\left(\frac{1}{2} \omega_{K} u_{\mathrm{rms}}(\Delta t)^{2}\right)^{2} \\
\Rightarrow & \quad\left(\gamma v_{0}\right)^{2} & >\left(\frac{1}{2} \omega_{K} u_{\mathrm{rms}}\right)^{2} .
\end{array}
$$

We hence define the vortical Stokes number with speed correction as

$$
S_{\omega, v} \equiv \mathcal{S}_{\omega} \frac{v_{0}}{u_{\mathrm{rms}}}=\frac{2 \gamma v_{0}}{\omega_{K} u_{\mathrm{rms}}}
$$

and global nematic order should occur for $\mathcal{S}_{\omega, v}>1$.

\subsubsection{Order parameters}

The orientational order of the system is described by three order parameters. They quantify the amount of nematic, polar, and chiral order in the system. Moreover, the smectic order parameter is used to investigate smectic order, i.e. layered structures of nematic order.

\section{Nematic order parameter}

The nematic order parameter is the natural choice in our system to describe global order due to the nematic symmetry of the inter-particle interaction. It is mostly used to describe liquid crystals and it is defined as the largest eigenvalue $S$ of the nematic order tensor

$$
\mathbf{Q} \equiv \frac{1}{2 N} \sum_{i=1}^{N}\left[3 \hat{e}_{i} \otimes \hat{e}_{i}-\mathbf{I}\right]
$$

where $\otimes$ denotes the tensor product and $\mathbf{I}$ is the unit tensor. The nematic order parameter is normalized in the following way: If all vectors $\hat{e}_{i}$ are aligned nematically, i.e. each two pairs are aligned either parallel or anti-parallel, the resulting nematic order parameter is $S=1$. In the other extreme, if there is no global directional order in the system, the nematic order parameter vanishes. The corresponding eigenvector, i.e. the vector $\hat{d}$ fulfilling $(\mathbf{Q}-S \mathbf{I}) \cdot \hat{d}=0$, is called the nematic director and denotes the main global direction along which the orientational vectors $\hat{e}_{i}$ are aligned (either parallel or anti-parallel).

It is sometimes useful to define the nematic order parameter and director locally. The definition uses a nematic order tensor analogous to Eq. (3.62) but using only a subset $N_{\alpha}$ of particles instead of $N$. These particles are usually contained in a 
sub-volume of the simulation box, for instance a slice perpendicular to a given axis. The resulting order parameter is called $S_{\mathrm{loc}}$ and the local director is $\hat{d}_{\mathrm{loc}}$.

\section{Smectic order parameter}

The investigated system can, in addition to nematic order, also possess smectic order. By this, we always denote a structure which is similar to the "Smectic $A$ " phase in liquid crystals (De Gennes and Prost, 1993, p. 18): The positional order shows a periodic structure in the direction of the nematic director because the particles are ordered in layers. Within each layer the positions of the particles are disordered. On the other hand, the orientations within each layer are nematically ordered with the director parallel to the layer normal. A system in the smectic $A$ phase can thus be described as a system with global nematic order and an additional density wave in the direction of the global director (McMillan, 1971). The smectic order parameter is defined as the maximum absolute value of the complex density waves when the periodicity is varied:

$$
S_{\text {smec }} \equiv \max \left(\left|\frac{1}{N} \sum_{j=1}^{N} \exp \left(i \frac{2 \pi}{L} \frac{\vec{x}_{j} \cdot \hat{d}}{\Delta_{n}}\right)\right|\right)_{n} .
$$

The positions $\vec{x}_{j}$ of the particles are taken into account as well as the global nematic direction $\hat{d}$. The periodicity $\Delta_{n}$ varies between 0 and 1 .

\section{Polar order parameter}

Even though the model being used in this work has nematic symmetry, it is sometimes useful to characterize the polar order of the system. We therefore define a polar order parameter as the average orientation in the system:

$$
P \equiv \frac{1}{N}\left|\sum_{i=1}^{N} \hat{e}_{i}\right|
$$

The direction of polarization is given by

$$
\hat{p}=\frac{1}{P} \frac{1}{N} \sum_{i=1}^{N} \hat{e}_{i}
$$

which is equal to the main global propagation direction of the system. 


\section{Chiral order parameter}

In this thesis, we will also deal with chirally ordered systems. By "chiral" system we mean that the orientations of particles are locally ordered nematically in planes but the local nematic director rotates as we move along the perpendicular axis of these planes (see Fig. 3.11a). To describe and especially quantify the degree of chiral order in a system is not as straightforward as the nematic or polar order in a system. A meaningful order parameter must include positions and orientations of the particles since chiral order means that particles which have a specific distance in space must have a specific angle between their orientations. A simple, pseudo-scalar combination of these quantities has been used by Memmer (2000) to define

$$
S_{221}\left(r^{*}\right)=-\sqrt{\frac{3}{10}}\left\langle\left[\left(\hat{e}_{i} \times \hat{e}_{j}\right) \cdot \hat{r}_{i j}\right]\left(\hat{e}_{i} \cdot \hat{e}_{j}\right)\right\rangle_{\left(r^{*}\right)_{i j}}
$$

where the average $\langle\cdot\rangle_{\left(r^{*}\right)_{i j}}$ is taken over all pairs at a distance $\left|\vec{r}_{i}-\vec{r}_{j}\right|=r^{*}$. We follow this approach and construct the chiral order parameter $S_{\chi}$ as

$$
S_{\chi} \equiv \frac{1}{S_{\chi, 0}} \frac{1}{N} \sum_{i=1}^{N}\left(-\frac{1}{N_{i}} \sum_{j=1}^{N_{i}}\left[\left(\hat{e}_{i} \times \hat{e}_{j}\right) \cdot \frac{\vec{r}_{i j}}{\left|\vec{r}_{i j}\right|}\right]\left(\hat{e}_{i} \cdot \hat{e}_{j}\right)\right)
$$

where $\vec{r}_{i j}=\vec{r}_{i}-\vec{r}_{j}$ and the second sum extends over all particles $j$ which are located within a distance of $r^{*}$ from the position of particle $i$. This approach differs from the idea of Memmer (2000) since it includes all particles inside a sphere instead of the particles in a spherical shell only. The advantage is that our chiral order parameter results in one single number for one configuration. However, as we will see below, the choice of the cutoff $r^{*}$ is important and our chiral order parameter will be tailored to one specific ratio of pitch and linear size of the simulation domain.

Most of the chiral structures that will be investigated have a pitch of $2 L$ : The local director of a slice perpendicular to the helical axis rotates by an angle of $\pi$ over the side length of the simulation domain. Therefore, a natural choice of the normalization constant $S_{\chi, 0}$ is such that a perfect chiral structure with a pitch of $2 L$ will result in $S_{\chi}=1$. The field of orientations (with the helical axis being parallel to $\hat{z}$, cf. Fig. 3.11a) can be described by

$$
e_{j}=\left(\begin{array}{c}
\cos \left(q z_{j}\right) \\
\sin \left(q z_{j}\right) \\
0
\end{array}\right), \text { with } q=\frac{\pi}{L}
$$

Because of the exact structure of this field, we do not have to take the first average in Eq. (3.67) and can choose the particle $i$. For convenience, we choose this particle to be at the center of the simulation domain $\left(\vec{r}_{i}=0, \hat{e}_{i}=\hat{x}\right)$. Instead of the sums in 


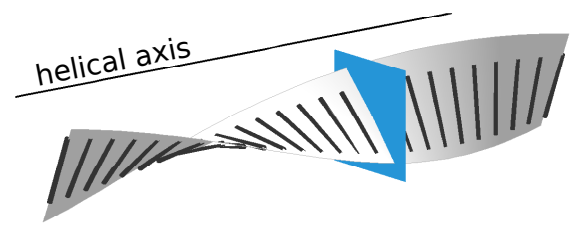

(a) Sketch of a perfect chiral pattern. The local nematic director (black line) rotates in the plane (blue) perpendicular to the helical axis as one moves along the helical axis.

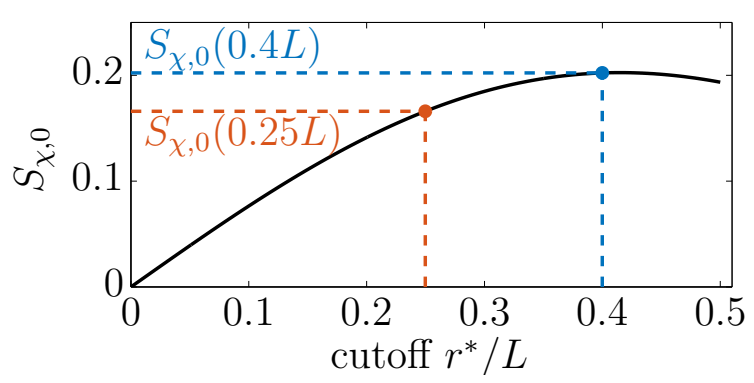

(b) Non-normalized chiral order parameter as a function of the cutoff for a perfect chiral structure (Eq. 3.70). A cutoff larger than $L / 2$ is not meaningful in a cubic simulation domain with periodic boundary conditions.

Figure 3.11: Calculation of the chiral order parameter.

the above definition we will use integrals and we will omit the subscript $j$. This leads to:

$$
\begin{aligned}
S_{\chi, 0} & =-\left\langle\left[\left(\hat{e}_{i} \times \hat{e}_{j}\right) \cdot \frac{\vec{r}_{i j}}{\left|\vec{r}_{i j}\right|}\right]\left(\hat{e}_{i} \cdot \hat{e}_{j}\right)\right\rangle \\
& =\left\langle z \sin (q z) \cos (q z) \frac{1}{\sqrt{x^{2}+y^{2}+z^{2}}}\right\rangle .
\end{aligned}
$$

The average is taken over a sphere with radius $r^{*}$ which is centered at $\vec{r}_{i}$. Its volume is hence $V=\frac{4}{3} \pi\left(r^{*}\right)^{3}$. We introduce spherical coordinates $(x=r \sin \theta \cos \phi, y=$ $r \sin \theta \sin \phi, z=r \cos \theta$ ). Equation (3.69) then becomes

$$
\begin{aligned}
S_{\chi, 0} & =\frac{1}{V} \int_{0}^{r^{*}} \mathrm{~d} r \int_{0}^{2 \pi} \mathrm{d} \phi \int_{0}^{\pi} \mathrm{d} \theta r^{2} \sin \theta z \frac{1}{2} \sin (2 q z) \frac{1}{r} \\
& =\frac{2 \pi}{2 V} \int_{0}^{r^{*}} \mathrm{~d} r \int_{0}^{\pi} \mathrm{d} \theta r^{2} \frac{\sin (2 \theta)}{2} \sin (2 q r \cos \theta) \\
& =\frac{\pi}{2 V} \int_{0}^{r^{*}} \mathrm{~d} r r^{2}\left[\frac{\cos \theta \cos (2 q r \cos \theta)}{q r}-\frac{\sin (2 q r \cos \theta)}{2(q r)^{2}}\right]_{\theta=0}^{\pi} \\
& =\frac{\pi}{2 V} \int_{0}^{r^{*}} \mathrm{~d} r r^{2}\left(\frac{\sin (2 q r)}{q^{2} r^{2}}-\frac{2 \cos (2 q r)}{q r}\right) \\
& =\frac{\pi}{2 V}\left(\frac{1}{q^{2}} \frac{1}{2 q}\left(1-\cos \left(2 q r^{*}\right)\right)-\frac{2}{q}\left(-\frac{1}{4 q^{2}}\left(1-\cos \left(2 q r^{*}\right)\right)+\frac{L}{8 q} \sin \left(2 q r^{*}\right)\right)\right) \\
& =\frac{3}{8\left(r^{*}\right)^{3} q^{2}}\left(\frac{1}{q}\left(1-\cos \left(2 q r^{*}\right)\right)-r^{*} \sin \left(2 q r^{*}\right)\right)
\end{aligned}
$$


(see Fig. 3.11b). We choose $r^{*}=L / 4$ which leads to

$$
q \frac{L}{2}=\frac{\pi}{2} \Rightarrow \cos \left(q \frac{L}{2}\right)=0, \quad \sin \left(q \frac{L}{2}\right)=1
$$

and hence

$$
\begin{aligned}
\Rightarrow S_{\chi, 0} & =\frac{\pi}{2 V}\left(\frac{1}{2 q^{3}}-\frac{2}{q}\left(-\frac{1}{4 q^{2}}+\frac{L}{8 q}\right)\right) \\
& =\frac{\pi}{2} \frac{48}{\pi L^{3}} \frac{L^{2}}{\pi^{2}}\left(\frac{L}{\pi}-\frac{L}{4}\right) \\
& =\frac{6}{\pi^{3}}(4-\pi) .
\end{aligned}
$$

We will thus use the following definition of the chiral order parameter:

$$
S_{\chi}=-\frac{\pi^{3}}{6(4-\pi)} \frac{1}{N} \sum_{i=1}^{N}\left(\frac{1}{N_{i}} \sum_{j=1}^{N_{i}}\left[\left(\hat{e}_{i} \times \hat{e}_{j}\right) \cdot \frac{\vec{r}_{i j}}{\left|\vec{r}_{i j}\right|}\right]\left(\hat{e}_{i} \cdot \hat{e}_{j}\right)\right)
$$

where the $N_{i}$ particles are all particles within a sphere centered at $\vec{r}_{i}$ with radius $L / 4$.

However, some of the chiral order parameters in Section 4.4 are calculated with a different cutoff of $r^{*}=0.4 L$ which corresponds to the maximum in $S_{\chi, 0}\left(r^{*}\right)$. The resulting normalization is

$$
S_{\chi, 0}^{\prime}=\frac{3}{8(0.4 \pi)^{3}}(1-\cos (0.8 \pi)-0.4 \pi \sin (0.8 \pi)) .
$$

The resulting chiral order parameter $S_{\chi}^{\prime}$ uses all particles in a sphere of radius $0.4 L$. Both chiral order parameters serve to distinguish a chiral from an achiral simulation.

\subsection{Analysis of clustering}

In Chapter 6 we will investigate the clustering of particles in a turbulent flow field. Clustering means that the local density of particles is enhanced compared to the global density and some kind of structure (e.g. patches) occurs. Three different measurement methods will be shown in the following: the patch enhancement factor, the analysis of possible giant number fluctuations, and the method of cluster analysis as it is used in the field of percolation. 


\subsubsection{Patch concentration enhancement factor}

The patch concentration enhancement factor (Durham et al., 2013) serves to quantify the increase of local density of particles as compared to randomly placed particles. To assign a local density to each particle site, the system is divided into Voronoi cells. The Voronoi cell is a generalization of the Wigner-Seitz cell used in solid-state physics. Given a particle, the Voronoi cell is the set of points that are closer to this particle than to any other particle in the system. The resulting cells are polyhedra. From the volume $v_{i}$ of the Voronoi cell, we can calculate the local particle density as $\rho_{i}=1 / v_{i}$ since there is only one particle inside this volume. By sorting the $v_{i}$ by magnitude and then taking the fraction $f$ of particles with smallest Voronoi volumes, we can calculate the mean density of the fraction $f$ of densest particles:

$$
C(f) \equiv\left\langle v_{i}\right\rangle_{i \in f}^{-1}
$$

This quantity is well-defined since

$$
C(f=1)=\left\langle v_{i}\right\rangle^{-1}=\left(\frac{1}{N} \sum_{i=1}^{N} v_{i}\right)^{-1}=\frac{N}{V}=\rho .
$$

The same measurement is also performed for a set of randomly placed particles and denoted by $C_{\text {random }}(f)$. The normalized patch concentration enhancement factor is hence given by

$$
Q(f) \equiv \frac{C(f)-C_{\text {random }}(f)}{\rho} .
$$

For $f \rightarrow 1$ the patch enhancement factor vanishes and (for point particles) it does not have an upper bound. It is always positive for clustered systems, but can have small negative values for approximately random systems. Therefore, we will always compute the absolute value keeping in mind that it is only different from $Q$ for the non-clustered systems with $|Q| \ll 1$.

The exact value of the patch concentration enhancement factor depends on the choice of $f$ which is in principle free to choose. A few examples how $Q$ changes with $f$ are given in Fig. 3.12. We see that the comparison of different simulations with one value of $f$ or another will almost always lead to the same conclusion, i.e. which system is more clustered than the other. However, the red curve in Fig. 3.12 shows that sometimes different values of $f$ can lead to different conclusions because the curves $Q(f)$ cross each other. This is important to keep in mind when different values of the patch concentration enhancement factor are compared. A short discussion about the possibility of an analytical expression for $C_{\text {random }}$ is included in Appendix A.1. 


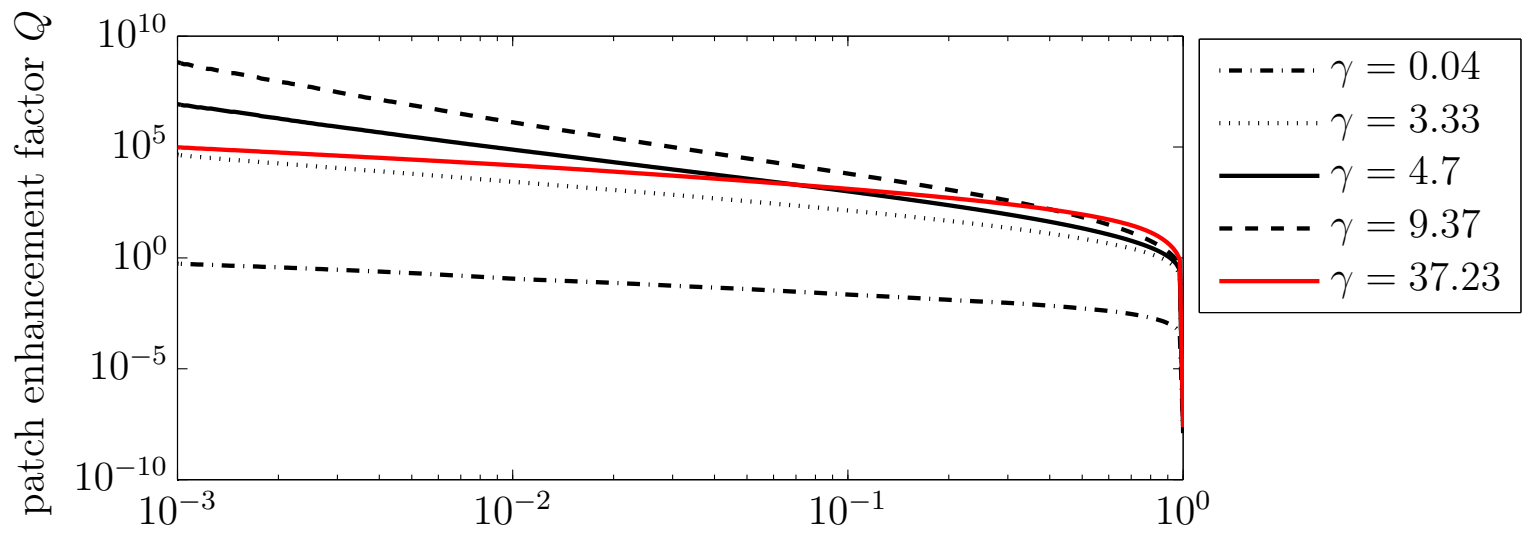

fraction $f$ of particles

Figure 3.12: Patch enhancement factor as a function of the fraction of particles considered. Different curves belong to different simulations of self-propelled particles in a turbulent field ( $\mathcal{P} \rightarrow \infty$, see Chapter 6 for detail) with differently strong clustering. The red curve belongs to one simulation which apparently behaves differently than the others.

\subsubsection{Number fluctuations}

A common method to analyze spatial segregation of particle-based systems is the analysis of number fluctuations: the system is divided into equally sized boxes and both the mean number of particles within each box $\langle n\rangle$ and the standard deviation $\Delta n=\sqrt{\left\langle n^{2}\right\rangle-\langle n\rangle^{2}}$ are calculated. This measurement is taken for differently sized boxes so that one obtains curves of $\Delta n$ vs. $\langle n\rangle$ (see Fig. 3.13 for two examples). If these curves show a clear power-law behavior $\Delta n \sim\langle n\rangle^{\alpha}$, which they do in our system, we can obtain the exponent $\alpha$ by a linear fit of $\log _{10}(\Delta n)$ vs. $\log _{10}(\langle n\rangle)$. In equilibrium one expects an exponent of $\alpha_{\text {equilibrium }}=0.5$ (see for example Mishra and Ramaswamy, 2006). Ramaswamy et al. (2003) showed that active nematics (like driven particles on a substrate) exhibit giant number fluctuations which means that the exponent $\alpha$ is larger than the equilibrium value. Moreover, they predicted the exponent to be

$$
\alpha_{\text {nematic }}=\frac{1}{2}+\frac{1}{d}
$$

for a system in $d$ dimensions.

\subsubsection{Cluster analysis}

A third method to quantify the clustering is based on a cluster analysis as it is done in the context of percolation theory. As the particles move off-lattice, we define that two 


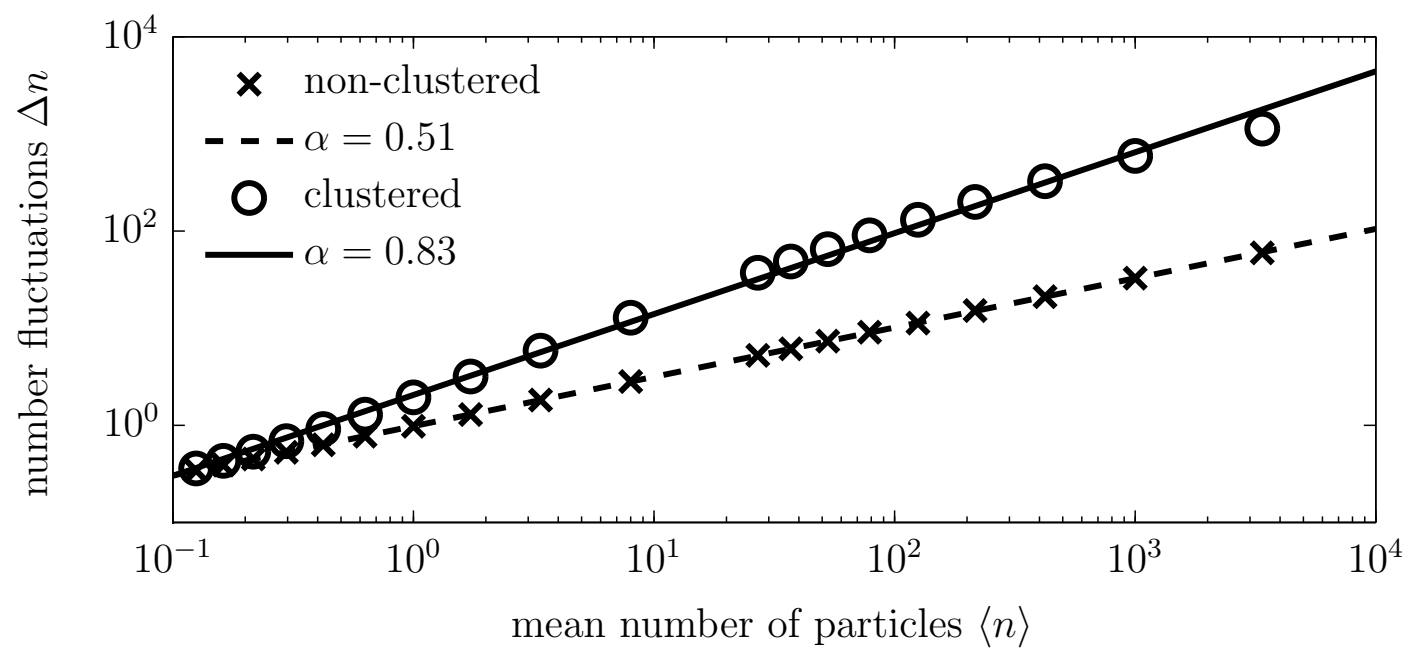

Figure 3.13: Two example curves for the analysis of number fluctuations: The measurements are given as symbols while the lines show the results of linear fits of $\Delta n \sim\langle n\rangle^{\alpha}$. The measurements in both examples show a power-law behavior. The first one $(x)$ belongs to a non-clustered system where the exponent is close to the equilibrium value of 0.5 . The second curve (o) shows giant number fluctuations with an exponent close to the theoretically predicted value of $5 / 6$ (figure to be published in Breier et al., 2017). 


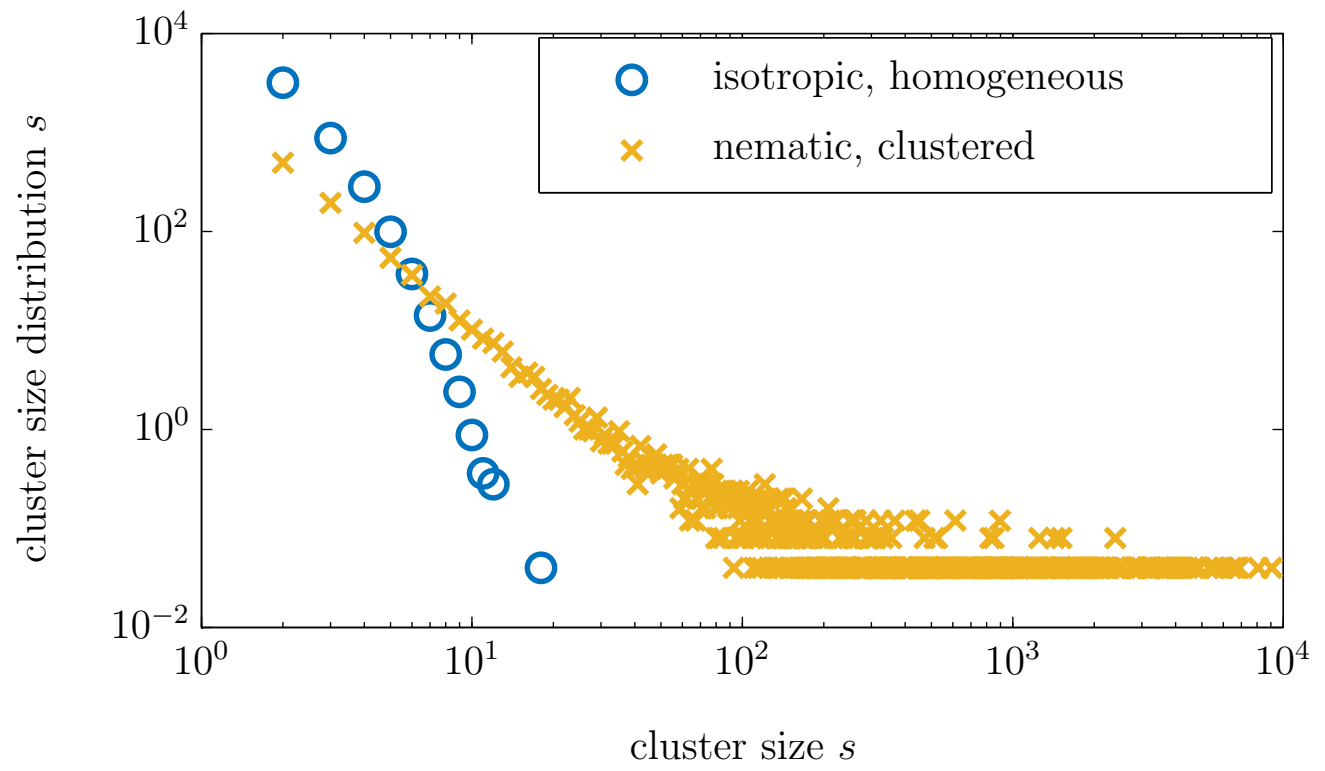

Figure 3.14: Typical examples of the cluster size distribution: The results of two different simulations are given. The blue circles correspond to a simulation in the isotropic phase without clustering while the yellow crosses represent a system in the nematic phase with clusters.

particles belong to the same cluster if their centers are closer than a cutoff distance $r_{c}$. In a given configuration of the system, the so-defined clusters are identified and their size $s$ is measured as the number of particles in a given cluster. The distribution of sizes is called the cluster size distribution $n(s)$. A significant result is obtained by averaging the cluster size distributions of several snapshots of the same system in the steady-state. Typical examples are given in Fig. 3.14. The cluster size distribution of a given system is (among others) characterized by the normalized second moment:

$$
N_{2} \equiv \frac{\sum_{s} n(s) s^{2}}{\sum_{s} n(s) s}
$$




\section{Structure formation by self-propelled point particles}

In this chapter we investigate our model of nematically aligning, self-propelled particles. It is described in detail in Section 3.1.1. The simulations are performed in a cubic domain with PBCs, if not stated otherwise. The typical input parameters are given in Tab. 4.1.

\begin{tabular}{ll}
\hline parameter & value \\
\hline number of particles & $N=66^{3}=287496$ \\
side lengths of simulations box & $L=L_{x}=L_{y}=L_{z}=\sqrt[3]{N / \rho}$ \\
nematic interaction range & $\epsilon=1$ \\
self-propulsion speed & $v_{0}=0.5$ \\
nematic relaxation constant & $\gamma=0.1$ \\
rotational diffusion & $D_{r}=\gamma / \mathcal{P}=\eta^{2} \Delta t / 2$ \\
time step & $\Delta t=0.1$ \\
\hline
\end{tabular}

Table 4.1: Input parameters for point-like self-propelled particles (if not stated otherwise). 


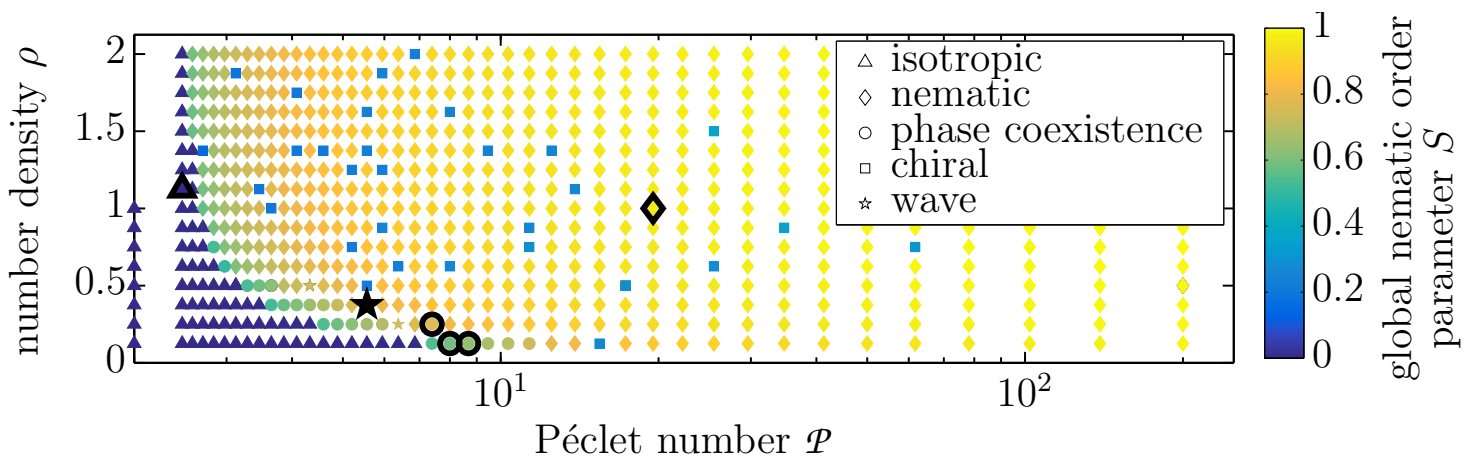

Figure 4.1: Nonequilibrium phase diagram of self-propelled point particles with nematic interaction. The global nematic order parameter $S$ is shown in color as a function of Péclet number and number density $\rho$. Different symbols refer to different states of the system as observed from the steady-state configurations. The bold symbols denote the systems from which the snapshots are given in Fig. 4.2 (graph modified from Breier et al., 2016).

\subsection{Phase diagram and snapshots}

The nonequilibrium phase diagram of the system is given in Fig. 4.1. The nematic order parameter in the steady-state is measured as a function of rotational Péclet number (Eq. 3.55) and number density $\rho=N / L^{3}$. The system is evolved until a steady state is very well established (typically more than $10^{5}$ time steps) and then the order parameters in the steady state are measured (typically averaged over another $10^{5}$ time steps). The nematic interaction range is the fundamental length scale in the system $(\epsilon=1)$ and hence the number density has the same value as the nondimensional number density $\rho \epsilon^{3}$. For a constant number density and increasing $\mathcal{P}$, we see a clear transition from the isotropic phase (denoted by $\triangle, S \rightarrow 0$ ) to the nematic phase (denoted by $\diamond, S \rightarrow 1$ ). Typical snapshots of these two phases are given in Figs. $4.2 \mathrm{a}$ and $4.2 \mathrm{~b}$.

The nematic and isotropic phases are separated by a rather narrow transitional domain in the phase diagram where coexistence of nematic and isotropic domains in the system is observed (denoted by $\circ$ ). Depending on the precise value of $P$, this phase coexistence can typically have three different shapes (see Fig. 4.2c): For the smallest value of $\mathcal{P}$ typically a nematically ordered, rather dense cylinder occurs in a dilute, isotropic gas. This cylinder percolates through the PBCs and the nematic director is aligned with the cylinder axis. When the Péclet number is slightly increased, the cylinder grows such that it connects itself also through a second dimension thus leading to a nematically ordered, dense layer in an isotropic, dilute surrounding. The local director within the layer is perpendicular to the layer normal and typically aligned along one of the box axes. Finally, for the largest values of $\mathcal{P}$ for which phase 


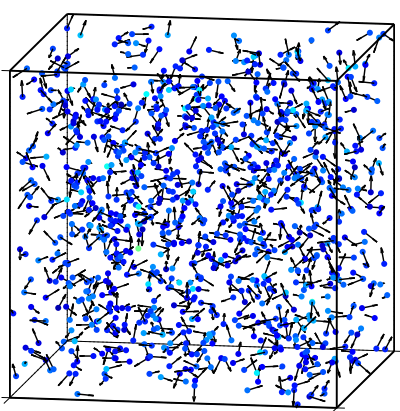

(a) Isotropic state $(\triangle, \mathcal{P}=2.47, \rho=1.125)$

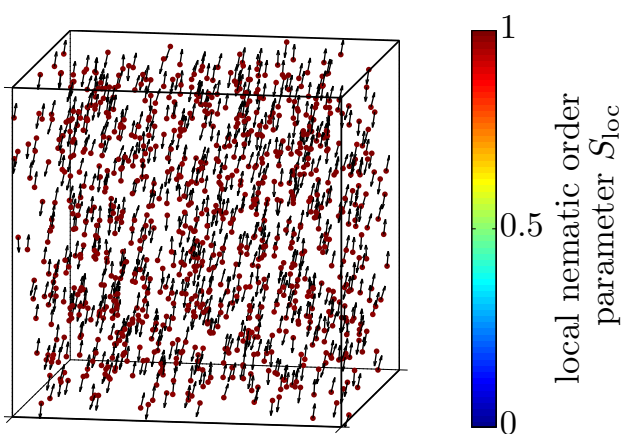

(b) Nematic state $(\diamond, \mathcal{P}=19.5, \rho=1)$
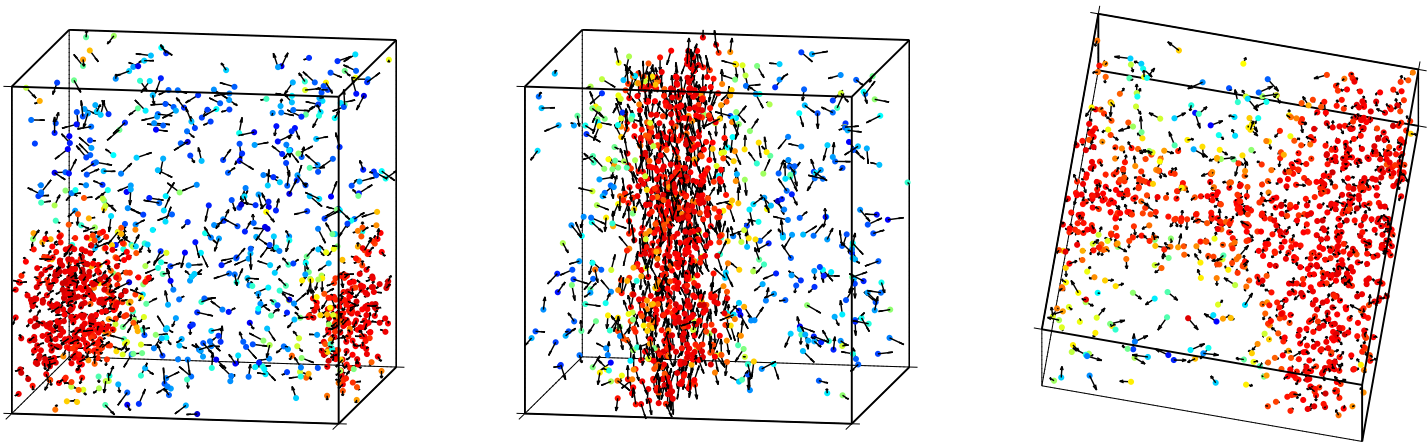

(c) Phase coexistence $(\circ, \mathcal{P}=[8 ; 8.68 ; 7.4], \rho=[0.125 ; 0.125 ; 0.25])$

Figure 4.2: Snapshots of the steady-state configurations of the self-propelled particles. The corresponding points are given by the bold symbols in Fig. 4.1. A small fraction $(0.35 \%)$ of the simulated particles is shown with the orientation vectors as small arrows. The simulation box is subdivided into $20^{3}$ boxes to calculate the local nematic order parameter $S_{\text {loc }}$ (represented by the color). 
coexistence is still visible, the emerging structure is the inverse of the first phase coexistence snapshot: Now a dilute, disordered cylinder is surrounded by a dense, nematically ordered surrounding. Here, the nematic director is again parallel to the cylinder axis. The cylinder axis (or the nematic director in the case of the nematic layer) is mostly aligned along one of the box axes in the examined snapshots of the phase coexistence state. This is an effect of the PBCs: When a nematically ordered cylinder forms, it can most easily connect to itself when it is aligned along one of the axis. This is a positive feedback because it stabilizes itself. In principle, however, the cylinder axis could be at any angle inside the box (but fulfilling the PBCs).

In the nematic phase (close to the isotropic-nematic phase transition) another structure of the particles was found: Polarized, dense waves which travel through the box (denoted by $\star$ ). We will discuss them in detail in Section 4.3.

Finally and most interestingly, a chiral structure occurs at different places in the nematic phase (see scattered squares $\square$ in Fig. 4.1). This is investigated in detail in Breier et al. (2016) and in Section 4.4.

\subsection{Isotropic-nematic transition}

The nonequilibrium phase diagram (Fig. 4.1) shows a very clear transition from an isotropic state (at low $\rho$ or low $\mathcal{P}$ ) to a nematic state. We observe that the locus of this isotropic-nematic transition is a function of both Péclet number and number density ${ }^{1}$. To quantify this further, we measure the critical Péclet number as a function of $\rho$. This critical Péclet number is located somewhere between the largest $\mathcal{P}$ which results in an isotropic state and the lowest $\mathcal{P}$ which leads to a nematic state. Here, the nematic state also includes states with local nematic order, i.e. wave, phase coexistence, and chiral. The resulting measured critical Péclet number as the mean of these two Péclet numbers (largest in isotropic state and lowest in nematic state) is given in Fig. 4.3. The blue crosses $(\times)$ correspond to the phase diagram in Fig. 4.1 where $\gamma$ was set to 0.1 while the other two symbols denote measurements from phase diagrams with $\gamma=0.5$ (red circles $\circ$ ) and (yellow triangles $\triangle$ ) $\gamma=1$, respectively. The critical Péclet number clearly decreases with number density. The three curves (which differ in relaxation constant) do not collapse for small number densities which is remarkable since the Péclet number includes the relaxation constant. To understand the functional dependency between critical Péclet number and density, we perform a mean-field theory with linear stability analysis in the following section.

${ }^{1}$ The influence of the time step $\Delta t$ is discussed in Appendix A.2 and possible finite-size effects are investigated in Appendix A.3 
number of particles in $\mathcal{B}_{\epsilon}$

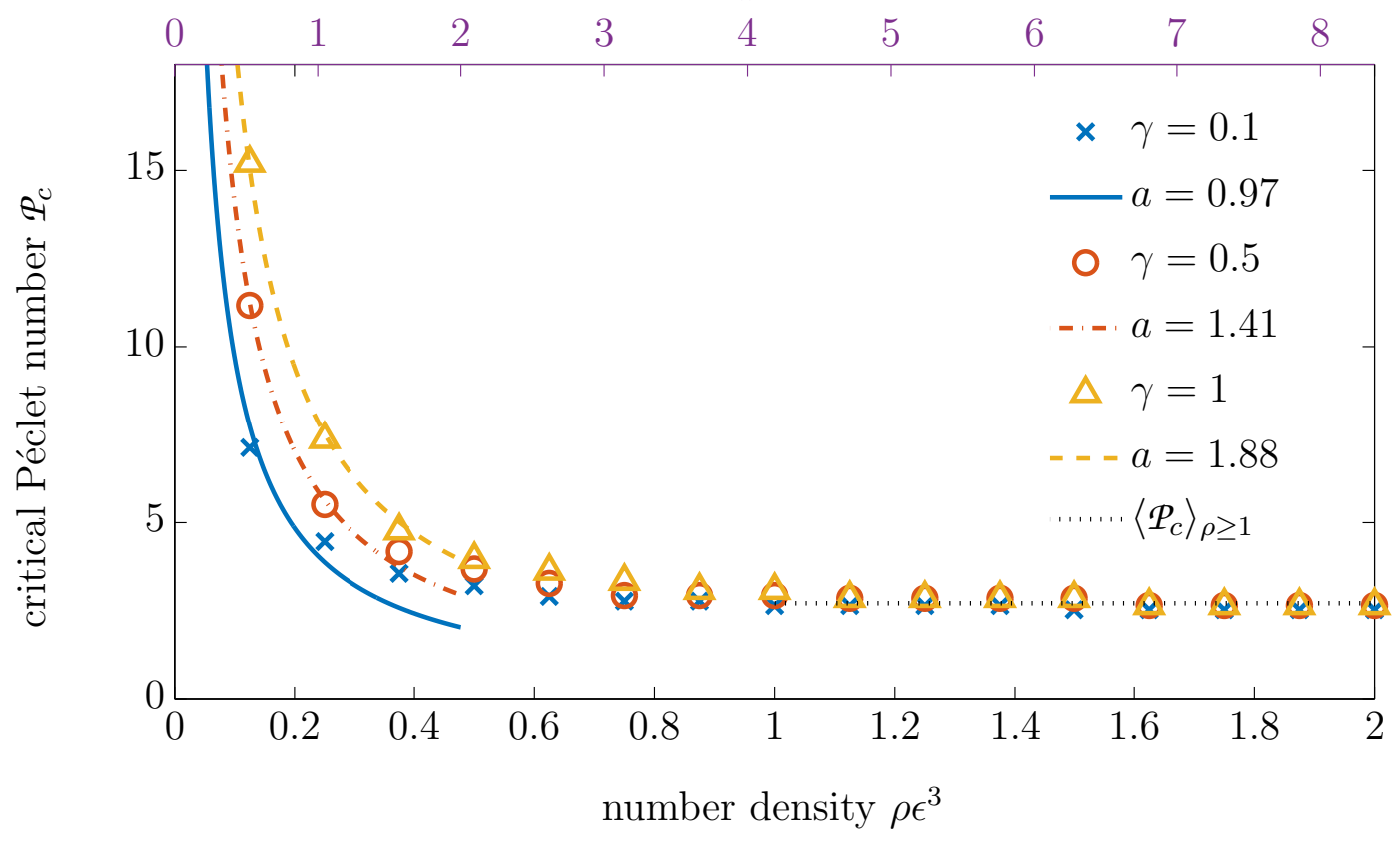

Figure 4.3: Critical Péclet number as a function of global number density and number of particles in the interaction sphere (volume $(4 / 3) \pi \epsilon^{3}$ ). Different colors correspond to simulations with different nematic relaxation constants $\gamma$. The symbols denote the measured critical Péclet number. The colored lines are the results of corresponding fits of the function $\mathcal{P}_{c}=a /\left(\rho \epsilon^{3}\right)$. The black dotted line is the mean critical Péclet number of all high-density simulations with $\rho>1$. 


\subsubsection{Mean-field theory}

Peruani et al. (2008) studied a very similar model to ours but in two dimensions. They developed a simple mean-field theory and applied the method of linear stability analysis to predict the locus of the isotropic-nematic transition. They found an onset of nematic order for

$$
\rho_{0}>\frac{4}{\pi \epsilon^{2}} \frac{D_{\theta}}{\gamma}
$$

where $\rho_{0}=N / L^{2}$ is the two-dimensional number density, $D_{\theta}$ is the rotational diffusion constant, and $\gamma$ and $\epsilon$ are the relaxation constant and the nematic interaction range. This implies (in our notation) a relationship between the critical Péclet number and the global density of the form $\mathcal{P}_{c} \propto \rho^{-1}$. In the following, we will apply the same approach to our model and derive an expression for the critical Péclet number.

We describe (in complete analogy to Peruani et al., 2008) our system by a probability density field $\psi(\vec{r}, \hat{e}, t)$. The particle density and the angular distribution are hence given by

$$
\begin{aligned}
\rho(\vec{x}, t) & =\oint \psi(\vec{x}, \hat{e}, t) \mathrm{d} \hat{e} \text { and } \\
C(\hat{e}, t) & =\int_{\Omega} \psi(\vec{x}, \hat{e}, t) \mathrm{d} \vec{x},
\end{aligned}
$$

where $\oint$ denotes the integral over the surface of the unit sphere and $\int_{\Omega}$ is the volume integral over the simulation domain. The corresponding evolution equation for $\psi$ is

$$
\partial_{t} \psi=D_{r} \Delta_{e} \psi-\nabla_{e}\left[\vec{F}_{e} \psi\right]-\nabla_{x}\left[\vec{F}_{x} \psi\right]
$$

with $\Delta_{e} \equiv \nabla_{e}^{2}$. The subscript of $\nabla$ denotes the variable with respect to which the function is differentiated. The deterministic fluxes $\vec{F}_{e} \psi$ and $\vec{F}_{x} \psi$ correspond to local nematic alignment and active migration, respectively. Integration of Eq. (4.2) with respect to $\vec{x}$ over the simulation domain leads to an equation for $C$ (under the assumption that differentiation with respect to $\hat{e}$ and integration are interchangeable):

$$
\begin{aligned}
\int_{\Omega} \partial_{t} \psi \mathrm{d} \vec{x} & =\int_{\Omega}\left(D_{r} \Delta_{e} \psi-\nabla_{e}\left[\vec{F}_{e} \psi\right]-\nabla_{x}\left[\vec{F}_{x} \psi\right]\right) \mathrm{d} \vec{x} \\
\Rightarrow \quad \partial_{t} C & =D_{r} \Delta_{e} C-\int_{\Omega}\left(\nabla_{e}\left[\vec{F}_{e} \psi\right]+\nabla_{x}\left[\vec{F}_{x} \psi\right]\right) \mathrm{d} \vec{x}
\end{aligned}
$$

We now need expressions for the deterministic fluxes. The flux corresponding to the active migration is given by

$$
\vec{F}_{x}=v_{0} \hat{e} .
$$

The flux corresponding to the nematic alignment mechanism can be calculated from 
the Lebwohl-Lasher potential (Eq. 3.1) as

$$
\vec{F}_{e}=-\gamma \int_{\mathcal{B}_{\epsilon}(\vec{x})} \oint\left[\nabla_{e} U_{\mathrm{LL}}\left(\vec{x}, \hat{e}, \vec{x}^{\prime}, \hat{e}^{\prime}\right)\right]_{\perp} \psi\left(\vec{x}^{\prime}, \hat{e}^{\prime}, t\right) \mathrm{d} \hat{e}^{\prime} \mathrm{d} \vec{x}^{\prime}
$$

where the Lebwohl-Lasher potential is rewritten in the form

$$
U_{\mathrm{LL}}\left(\vec{x}, \hat{e}, \vec{x}^{\prime}, \hat{e}^{\prime}\right)= \begin{cases}U_{\mathrm{LL}}\left(\hat{e}, \hat{e}^{\prime}\right)=-\left(\hat{e} \cdot \hat{e}^{\prime}\right)^{2}, & \text { if }\left|\vec{x}-\vec{x}^{\prime}\right| \leq \epsilon \\ 0, & \text { otherwise }\end{cases}
$$

and $\mathcal{B}_{\epsilon}(\vec{x})$ is the interaction volume around $\vec{x}$. The subscript $\perp$ again refers to the component perpendicular to $\hat{e}$ such that

$$
\left[\nabla_{e} U_{\mathrm{LL}}\left(\hat{e}, \hat{e}^{\prime}\right)\right]_{\perp}=-2\left(\left(\hat{e} \cdot \hat{e}^{\prime}\right) \hat{e}^{\prime}-\left(\hat{e} \cdot \hat{e}^{\prime}\right)^{2} \hat{e}\right)
$$

Moreover, we assume a homogeneous distribution of particles:

$$
\psi(\vec{x}, \hat{e}, t)=C(\hat{e}, t) \frac{\rho}{N} .
$$

By inserting Eqns. (4.4), (4.5), and (4.8) into Eq. (4.2), we find the following equation for the angular distribution:

$$
\begin{aligned}
\partial_{t} C & =D_{r} \Delta_{e} C-\int_{\Omega}\left(\nabla_{e}\left[\vec{F}_{e} C\right] \frac{\rho}{N}+\nabla_{x}\left[\vec{F}_{x}\right] C \frac{\rho}{N}\right) \mathrm{d} \vec{x} \\
& =D_{r} \Delta_{e} C-\int_{\Omega} \nabla_{e}\left(\vec{F}_{e} C\right) \frac{\rho}{N} \mathrm{~d} \vec{x} \\
& =D_{r} \Delta_{e} C+\frac{\rho}{N} \int_{\Omega} \nabla_{e}\left[\left(\gamma \int_{\mathcal{B}_{\epsilon}(\vec{x})} \oint\left(\nabla_{e} U_{\mathrm{LL}}\right) C\left(\hat{e}^{\prime}\right) \frac{\rho}{N} \mathrm{~d} \hat{e}^{\prime} \mathrm{d} \vec{x}^{\prime}\right) C(\hat{e})\right] \mathrm{d} \vec{x} \\
& =D_{r} \Delta_{e} C+\frac{4}{3} \pi \gamma \frac{\epsilon^{3}}{L^{3}} \nabla_{e}\left[\left(\oint\left[\nabla_{e} U_{\mathrm{LL}}\right]_{\perp} C\left(\hat{e}^{\prime}\right) \mathrm{d} \hat{e}^{\prime}\right) C(\hat{e})\right] .
\end{aligned}
$$

We can simplify this equation by the transformations

$$
\tau=\frac{4}{3} \pi \gamma \frac{\epsilon^{3}}{L^{3}} t \quad \text { and } \quad D_{r}^{\prime}=D_{r}\left(\frac{4}{3} \pi \gamma \frac{\epsilon^{3}}{L^{3}}\right)^{-1},
$$

so that the equation for $C$ becomes

$$
\partial_{\tau} C=D_{r}^{\prime} \Delta_{e} C+\nabla_{e}\left[\left(\oint\left[\nabla_{e} U_{\mathrm{LL}}\right]_{\perp} C\left(\hat{e}^{\prime}\right) \mathrm{d} \hat{e}^{\prime}\right) C(\hat{e})\right] .
$$


We want to perform a linear stability analysis and therefore use the ansatz

$$
C(\hat{e})=C^{*}+C_{0} \exp (\lambda \tau) F(\hat{e})
$$

with $C^{*}=N /(4 \pi)$. If the real part of $\lambda$ is larger than zero, an instability occurs which corresponds to the onset of nematic order in our case. We will discuss the appropriate functions $F$ in detail below. Now we insert this ansatz into Eq. (4.11), use the fact that $\oint\left[\nabla_{e} U_{\mathrm{LL}}\right]_{\perp} \mathrm{d} \hat{e}^{\prime}=0$ and keep only terms which are linear in $C_{0}$. The resulting eigenvalue problem is

$$
\begin{aligned}
\lambda F(\hat{e}) & =D_{r}^{\prime} \Delta_{e} F(\hat{e})+C^{*} \nabla_{e} \oint\left[\nabla_{e} U_{\mathrm{LL}}\right]_{\perp} F\left(\hat{e}^{\prime}\right) \mathrm{d} \hat{e}^{\prime} \\
& =D_{r}^{\prime} \Delta_{e} F(\hat{e})+2 C^{*} \oint\left(5\left(\hat{e} \cdot \hat{e}^{\prime}\right)^{2}-1\right) F\left(\hat{e}^{\prime}\right) \mathrm{d} \hat{e}^{\prime}
\end{aligned}
$$

where integration and differentiation commute because they refer to different variables and we inserted the potential in Eq. (4.7). The function $F$ depends on the orientation vector $\hat{e}$ which is normalized, so we can use spherical coordinates and write $F(\hat{e})=$ $F(\theta, \phi)$. The Laplace operator in spherical coordinates can be separated into a radial and an angular part. Due to the normalization of $\hat{e}$ the Laplacian $\Delta_{e}$ consists only of the angular part. We use the eigenfunctions of this angular part of the Laplacian as an ansatz for $F$. They are given by the spherical harmonics

$$
Y_{l m}(\theta, \phi)=N_{l m} P_{l m}(\cos \theta) \exp (\mathrm{i} m \phi)
$$

with the normalization $N_{l m}$ and the associated Legendre polynomials $P_{l m}(x)$. The corresponding eigenvalues are $-l(l+1)$. The degree $l$ and the order $m$ are integers with $l \geq 0$ and $-l \leq m \leq l$. A long but straightforward calculation yields the result that this are indeed the eigenfunctions of the problem in Eq. (4.13) with the following eigenvalues:

$$
\lambda= \begin{cases}\frac{16 \pi}{3} C^{*} & \text { for } l=0, \\ -2 D_{r}^{\prime} & \text { for } l=1, \\ -6 D_{r}^{\prime}+\frac{16 \pi}{3} C^{*} & \text { for } l=2, \\ -D_{r}^{\prime} l(l+1) & \text { for } l>2 .\end{cases}
$$

The $l=0$ eigenfunction yields a positive eigenvalue but corresponds to the isotropic distribution. The $l=1$ eigenfunction as well as all eigenfunctions with $l>2$ correspond to negative eigenvalues and thus do not lead to an instability. The onset of nematic order is hence given by the $l=2$ eigenfunction which leads to

$$
-6 D_{r}^{\prime}+\frac{16 \pi}{3} C^{*}>0
$$


The corresponding set of spherical harmonics shows nematic symmetry. Reinserting Eq. (4.10) and $C^{*}=N /(4 \pi)$ yields as a final result

$$
\rho \epsilon^{3} \frac{16 \pi}{3}>18 \frac{D_{r}}{\gamma}=18 \frac{1}{\mathcal{P}}
$$

and thus for the critical Péclet number

$$
\mathcal{P}_{c}=\frac{54}{16 \pi} \frac{1}{\rho \epsilon^{3}} \approx 1.07 \frac{1}{\rho \epsilon^{3}}
$$

which is the same functional dependency between critical Péclet number and density as in the two-dimensional case.

\subsubsection{Comparison to data}

The measured critical Péclet number decreases as a function of number density (see Fig. 4.3). However, the curves approach a plateau for large number densities. This clearly indicates a discrepancy between the mean-field theory (with the result $\mathcal{P}_{c} \propto$ $\rho^{-1}$ ) and the outcome of our simulations. The reason lies in the additional factor $1 / n_{i}$ in the Lebwohl-Lasher potential of the agent-based model (see Eq. 3.1) which has not been incorporated into the mean-field theory. We therefore distinguish two different regimes of our system according to the global density.

The low-density regime is valid for densities which lead to up to two particles in a sphere $\mathcal{B}_{\epsilon}$ of radius $\epsilon$ on average so that each particle finds up to one neighbor in its interaction volume. In this regime the number of neighbors does not have to be taken into account and we can fit the result of the mean-field theory to the measurements from the agent-based simulations. We find a good qualitative agreement in the functional form of $\mathcal{P}_{c}$ as a function of $\rho$. However, the resulting fit parameters $a$ vary for the three different curves corresponding to different values of the nematic relaxation constant $\gamma$, though the agreement with mean-field theory is good, all considered. It is closest to the theoretical value of $54 /(16 \pi) \approx 1.07$ for $\gamma=0.1$ and deviates for larger $\gamma$. The reason could be that we assumed a homogeneous distribution of particles in the mean-field approach which is not necessarily valid in the MD simulations. Larger values of the nematic relaxation constant mean faster alignment which favors clustering because particles possibly fully align during contact. We therefore expect increasing deviations from homogeneity for increasing $\gamma$ which matches to larger deviations from the result of the mean-field theory.

The mean-field theory cannot be applied in the high-density regime $\left(\rho \epsilon^{3}>1\right)$ because the presence of the factor $1 / n_{i}$ in the potential results in an independence of the interactions from the actual number of neighbors. Effectively it leads to an average of all the interactions one particle encounters with its neighbors and hence 


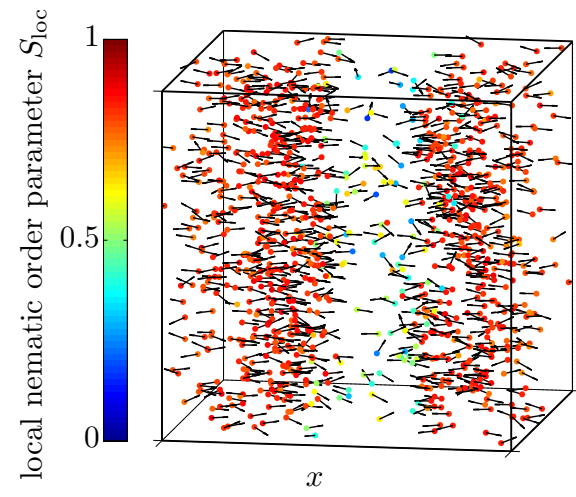

(a) Snapshot of a wave propagating through the system. For the sake of clarity, only $0.35 \%$ of the particles shown with their orientation as little arrow. The local nematic order parameter (color) is calculated from a subdivision of the simulation domain into $20^{3}$ boxes.

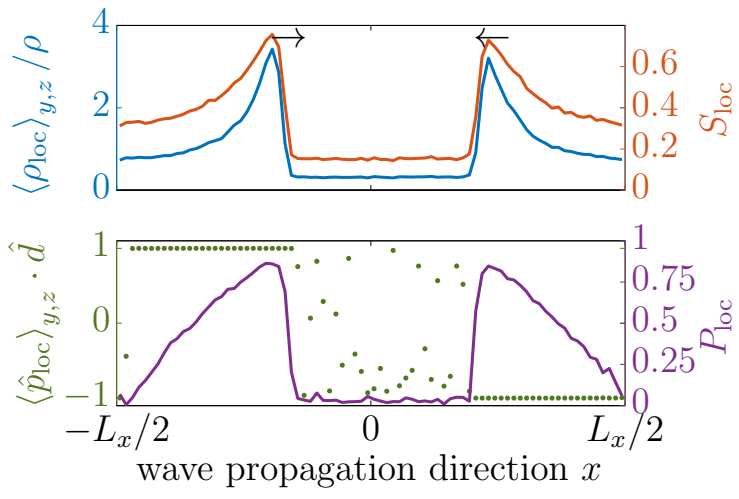

(b) Profiles measured along the wave propagation direction. Upper panel: Normalized local density (blue) and local nematic order parameter (orange). Lower panel: Scalar product between local polarization direction and global director (green) and local polarization (purple).

Figure 4.4: Characterization of the wave phase (indicated by $\star$ in Fig. $4.1, \mathcal{P}=5.56$, $\rho=0.375)$.

the critical Péclet number is independent of the number density. We find an average critical Péclet number for densities $\rho \geq 1$ of $\left\langle\mathcal{P}_{c}\right\rangle_{\rho \geq 1}=2.711$.

\subsection{Waves}

In the nematic phase (but rather close to the isotropic-nematic transition, see Fig. 4.1) the system exhibits waves of nematically aligned particles propagating through the 3D domain. These waves are investigated in this section. A typical snapshot of a wave, as well as profiles of density and order parameters are given in Fig. 4.4. This example shows a rather high global nematic order and areas of high local nematic order as well. In the middle of the simulation box a disordered, dilute gas is visible. Visual inspection of the time evolution ${ }^{2}$ reveals that in fact two polarized wavecrests counterpropagate along the x-axis. Figure $4.4 \mathrm{~b}$ shows four different measurements which are averaged within slices perpendicular to the wave propagation direction $x$ : density, nematic order parameter, alignment of polarization with the nematic director, and local polarization. The normalized local density shows two non-symmetric wavecrests with a sharp front and an elongated tail. The profile of the local nematic order

\footnotetext{
${ }^{2}$ see attached movie wave.avi
} 
parameter is very similar in shape to the density profile with highest nematic order at the points of highest local density. These two profiles only prove that we have areas of high local density which are even nematically ordered. To prove that the snapshot really consists of a wave-like structure, we study the spatial dependence of the polarization. The local polarization shows (similarly to the nematic order) a maximum where the local density is highest. The shape of the polarization profile is different from the nematic order and density profiles within a roughly linear increase of $P_{\text {loc }}$ from the tail to the front of the wave. Finally, we investigate the scalar product between the direction of local polarization (averaged over a slice perpendicular to $\hat{x}$ ) and the global nematic director ${ }^{3}$. This scalar product shows that the particles in the left side of the box $\left(-0.5 L_{x} \leq x \leq-0.15\right)$ are mostly aligned along $\hat{x}$ while the particles with $0.15 \leq x \leq 0.5 L_{x}$ have orientations on average parallel to $-\hat{x}$. The direction of the local polarization in the dilute part of the box $\left(-0.15 L_{x} \leq x \leq\right.$ $\left.0.15 L_{x}\right)$ does not have a preferred direction and the local order is very low.

The temporal inspection of the order parameters (Fig. 4.5) reveals that the wave pattern evolves over a long time span compared to, for instance, the formation of the nematic, homogeneous state. From the example shown in Fig. 4.5, we see that the system evolves global nematic order rather quickly; the maximum in $S$ is very well established at $t \approx 7500$. The full transient takes roughly $7 \times 10^{4}$ time units, during which the nematic order parameter decreases slightly, the polarization exhibits a small maximum, and the smectic order parameter oscillates and increases in both mean value and amplitude. However after this transient, the smectic order parameter shows very pronounced oscillations. The main period of this oscillation matches the value of $L_{x} / v_{0}$ which is the time it takes one particle to travel once across the simulation domain.

The wavelength is essentially set by the orientation of the wave within the simulation domain together with the influence of the PBCs. We derive the wavelength in two dimensions which can then be easily extended to three dimensions, and we restrict ourselves to a square box. We assume the wavefront to be placed in the middle of the simulation domain at some angle (see the blue line in Fig. 4.6a). The wavefront is oriented perpendicular to the nematic director $\hat{d}$ which is parallel to the wave propagation direction as we saw before. The equation which describes all points on the wavefront is

$$
\vec{x} \cdot \hat{d}=0 .
$$

The wavefront crosses the boundary at the point $\vec{x}_{A}$. Assuming that it crosses the

\footnotetext{
${ }^{3}$ In fact, the global nematic director is almost perfectly parallel to $\hat{x}$.
} 


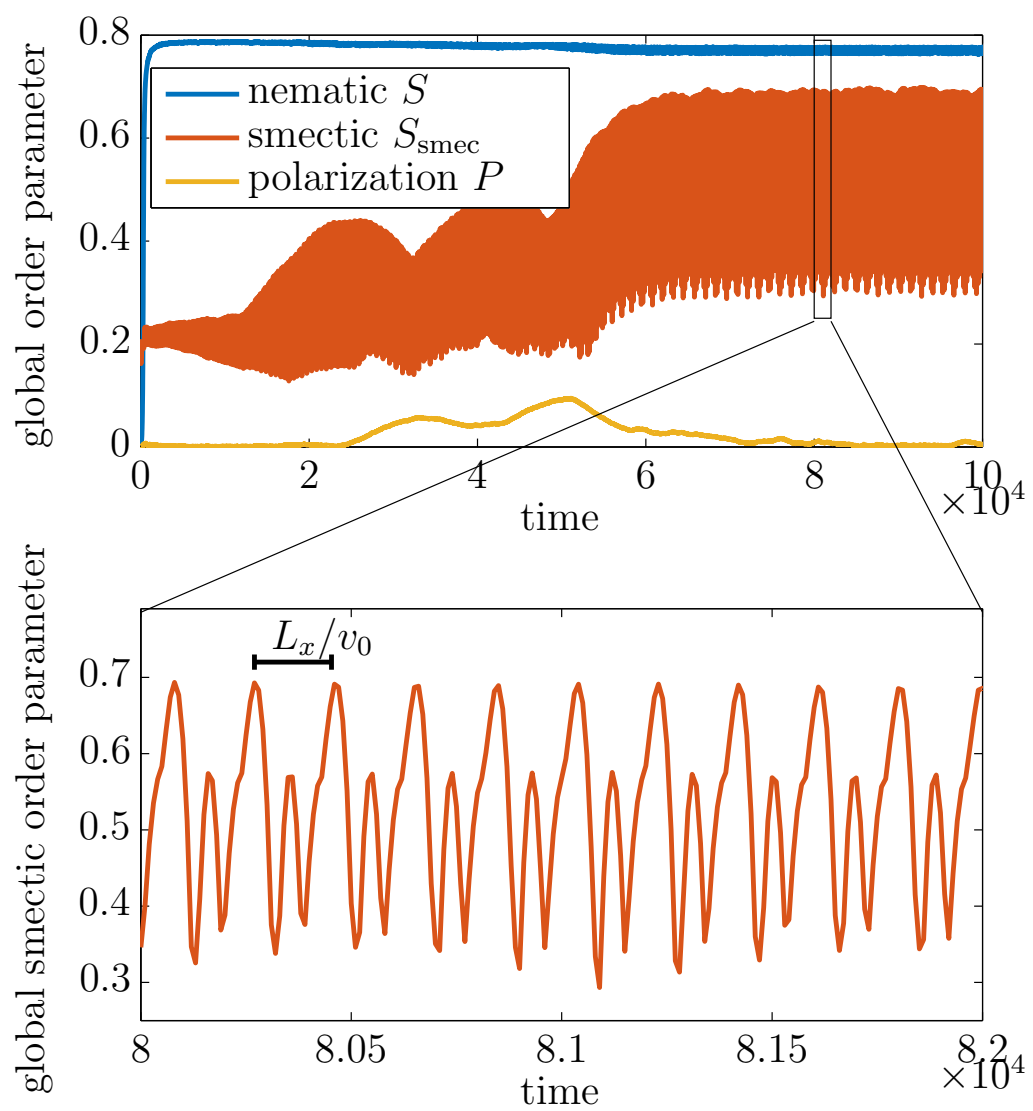

Figure 4.5: Temporal evolution of the order parameters of a simulation which eventually shows the wave pattern (Fig. 4.4). The upper panel shows the global order parameters as a function of time. The lower panel is a zoom into a short time window to see the short-term variations in the smectic order parameter. 


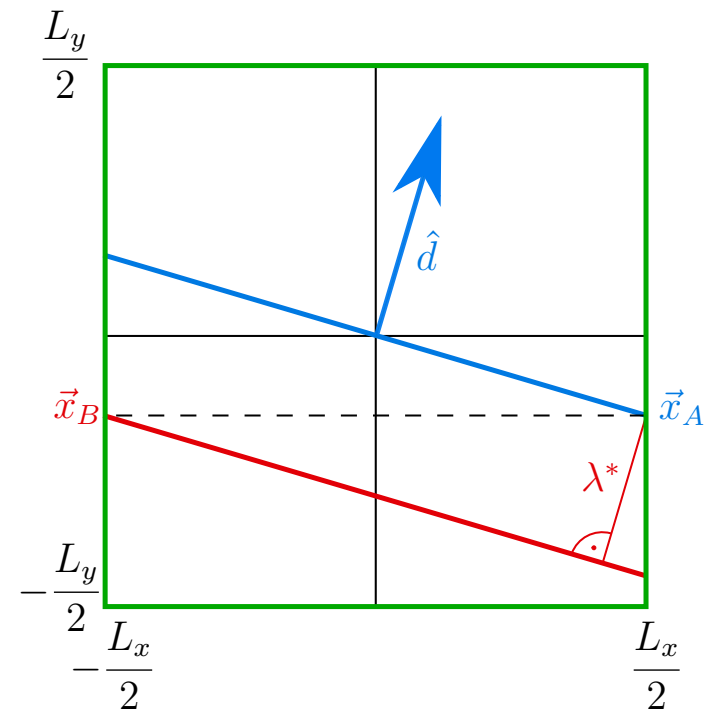

(a) Simulation domain. The "first" wavefront is given in blue, its continuation (through PBCs) is denoted in red.

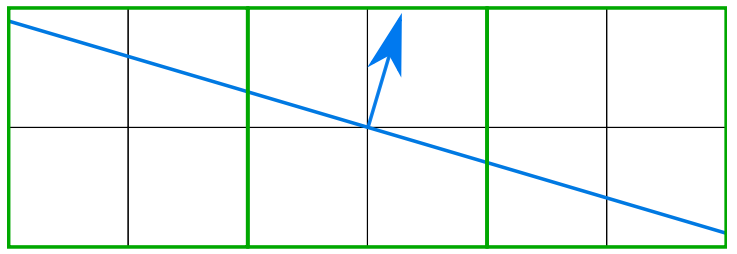

(b) The simulation domain is shown in the middle with two copies to the left and to the right.

Figure 4.6: Sketch for the wave in a system with PBCs.

$y$-axis first (as in the sketch), the position is given by

$$
\begin{array}{rlrl}
\vec{x}_{A} \cdot \hat{d} & =0 \\
\Rightarrow \quad \frac{L_{x}}{2} d_{x}+y d_{y} & =0 \\
\Rightarrow & y & =-\frac{L_{x}}{2} \frac{d_{x}}{d_{y}} .
\end{array}
$$

Because of PBCs, the wavefront also has to go through the point $\vec{x}_{B}$ (see sketch) which possesses the same $y$-coordinate as $\vec{x}_{A}$. To calculate the distance $\lambda^{*}$ we construct a line through $\vec{x}_{B}$ in the direction $\hat{d}$ and calculate the crossing with the first wavefront:

$$
\begin{aligned}
\vec{x} & =\vec{x}_{B}+\lambda^{*} \hat{d} \\
\Rightarrow \quad \vec{x} \cdot \hat{d} & =\vec{x}_{B} \cdot \hat{d}+\lambda^{*} \\
\Rightarrow \quad \lambda^{*} & =-\vec{x}_{B} \cdot \hat{d} \\
& =-\frac{L_{x}}{2} d_{x}-\frac{L_{x}}{2} \frac{d_{x}}{d_{y}} d_{y}=-L_{x} d_{x} .
\end{aligned}
$$

In the second step, we used the fact that $\vec{x} \cdot \hat{d}=0$ (condition for the first wavefront). 
This distance $\lambda^{*}$ is not necessarily the true wavelength because this depends on how often the PBCs have to be applied until the wavefront percolates. We can quantify this and calculate the true wavelength by considering the possible wave propagation directions in the following. The PBCs restrict the orientation of the wavefront inside the simulation box. The boundary conditions mean that the full (in this case two dimensional) space is covered by squares which are copies of the simulation box (see Fig. 4.6b). This means that the wavefront which goes through the center of the simulation box also has to cross the center of one copy. This can only be achieved if the nematic director is given by

$$
\hat{d}=\frac{\vec{n}}{|\vec{n}|} \quad \text { with } \vec{n} \in \mathbb{N}_{0}^{2}
$$

The true wavelength is given by

$$
\lambda=\frac{L}{n}
$$

if we assume that the vector $\vec{n}$ is the smallest possible vector (e.g. $(3 ; 2 ; 0)$ instead of $(6 ; 4 ; 0))$. This can be understood as the "greatest common divisor" and the equivalent in $y$-direction $\left|\lambda^{* *}\right|=L_{y} d_{y}$. The same arguments hold in three dimensions and the possible directors are given by Eq. (4.22) with the corresponding wavelengths in Eq. (4.23). It is important to note that the natural numbers which form the director should not be too large because otherwise the individual wavefronts might not be distinguishable from each other. Moreover, several waves can form in the same simulation thus leading to a smaller effective wavelength.

We can verify this derivation of the wavenumber by comparing the measured wavelength of a pattern with the calculated wavelength. The latter is derived from the global nematic director. To do so, we calculate $\hat{d}$ as well as the ratios of each pair of components. These ratios then lead to the underlying vector $\hat{n}$ with integer components. On the one hand, this vector allows us to calculate the largest possible wavelength $\lambda_{c}$ following Eq. (4.23). On the other hand, we can also measure the wavelength from the density profile along the global director. To obtain this density profile, we first measure the local density in cubic subboxes of the simulation domain and then rotate the coordinate system such that one axis of the new coordinate system is parallel to the global nematic director. It is then straightforward to calculate the mean local density in slices perpendicular to the director (just like in Fig. 4.4b). We measure the wavelength $\lambda_{m}$ by a simple measurement of the distance between two peaks. However, since the system may be composed of several wavetrains (coor counter-propagating), this measurement is not unambiguous and leads to several possible wavelengths. Nevertheless, the comparison between $\lambda_{c}$ and $\lambda_{m}$ for 40 inde-

${ }^{4}$ The greatest common divisor is defined for natural numbers. But since the components of the director are constructed from natural numbers (and then normalized), we use an analogue here. 


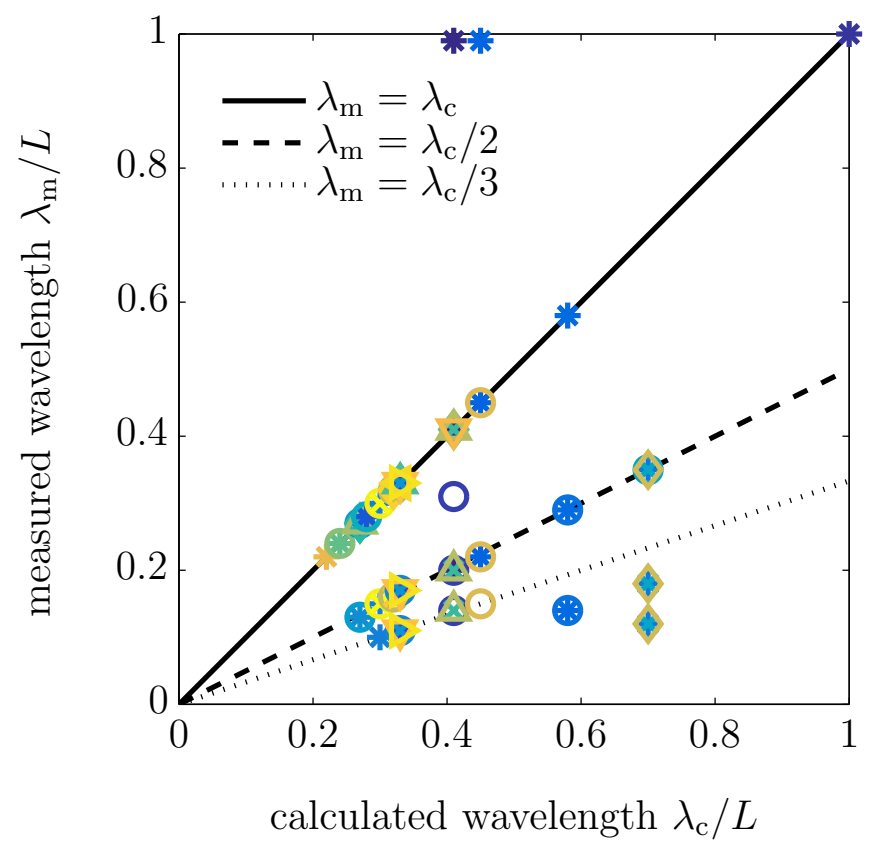

Figure 4.7: Measured $\lambda_{\mathrm{m}}$ vs. calculated $\lambda_{\mathrm{c}}$ wavelength for 40 wave patterns (different relaxation constants, different Péclet numbers). Each color represents one single simulation, different symbols are used to distinguish simulations with the same values of $\lambda_{c}$. 


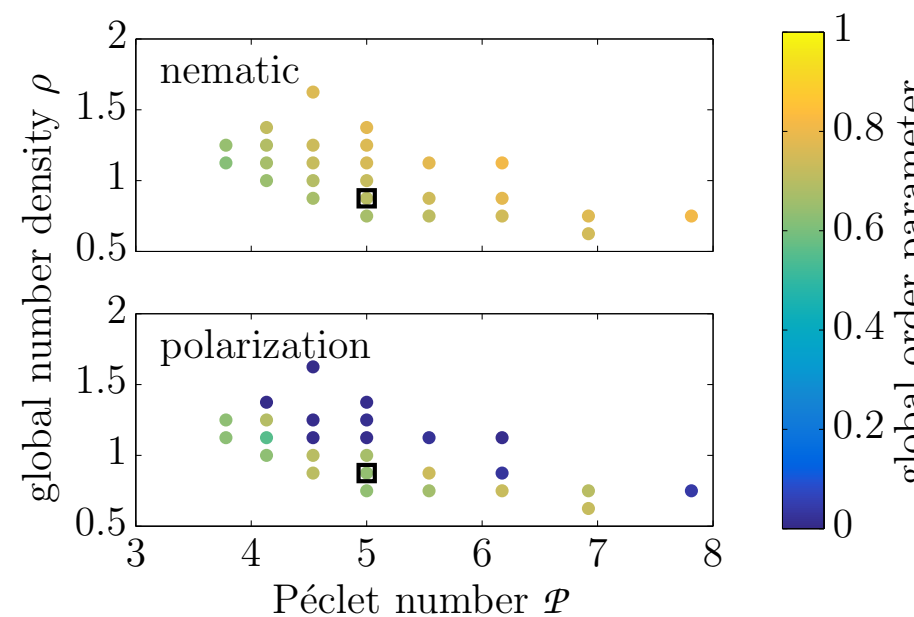

(a) Global nematic order parameter and polarization of wave patterns in the $(\rho, \mathcal{P})$ plane. (b) Snapshot of a globally polarized wave $(\mathcal{P}=5, \rho=0.875)$ rendered like in Fig. 4.1.

Figure 4.8: Detailed investigation of the region in the phase diagram where polarized waves occur (here: $\gamma=1$ ).

pendent wave patterns, shown in Fig. 4.7 reveals a very good agreement between the two approaches. The measured wavelength either matches the calculated one directly, or one half, one third, ... of it which is then due to several wavetrains. In two cases $\lambda_{m}$ is much larger than $\lambda_{c}$ and almost equal to the box sizes. The reason could be two counter-propagating waves which can (in our measurement) lead to any wavelength depending on the exact time when the measurement is taken. To conclude we find that the maximum possible wavelength is indeed determined geometrically by the orientation of the global nematic director. However, shorter wavelengths are observed repeatedly.

Finally, we can investigate the global order parameters of the wave pattern in the phase diagram. Since the phase diagram region in which waves occur is very small in the case of a low nematic relaxation constant $(\gamma=0.1$, Fig. 4.1), we analyze simulations with a larger relaxation constant $(\gamma=1)$. Here, the wave region in the phase diagram is larger (see Fig. 4.8a). The global nematic order parameter increases within the wave region when moving from the isotropic phase (low $\mathcal{P}$, low $\rho$ ) to the nematic phase (high $\mathcal{P}$, high $\rho$ ). However, the global polarization indicates that the wave region is in fact separated into two sub-regions. Close to the nematic phase, the polarization vanishes as expected for our system with nematic symmetry. However, close to the isotropic phase the polarization reaches surprisingly high values of $P \simeq 0.7$ which are close to the values of the nematic order parameter in this region of the phase diagram. Hence, the system is not only locally polarized (inside the 
wave) but also globally. The corresponding snapshots (see Fig. 4.8b for an example) show a polarized wave as before but without a counter-propagating antagonist.

\subsection{Spontaneous chiral symmetry breaking}

In the nematic region of the nonequilibrium phase diagram (Fig. 4.1) we observe the formation of chiral structures which seem to occur spontaneously and are apparently not a "phase" which could be localized precisely in the phase diagram. This chiral pattern is investigated in this section. The main results discussed in this section were published in Breier et al. (2016).

\subsubsection{Importance of chirality}

An object or configuration of a specific chirality or handedness is distinguishable from its mirror image. This is an important property of many ingredients of life like amino acids, sugar, and -most prominently- DNA. It is hence important to understand how a chiral structure can emerge in an otherwise achiral system. Furthermore, homochirality is found for many of these objects; only one of the enantiomers (image or mirror image) occurs naturally. For example, amino acids are left-handed, while most sugars occur in the right-handed form only and so does most of the DNA (Meierhenrich, 2008). The investigation of this (homo-)chirality is an active field of research since it appears to be a key ingredient to understand how life formed.

Classical examples of chiral symmetry breaking in achiral systems include the Belousov-Zhabotinskii reaction and the formation of so-called Liesegang rings (Meierhenrich, 2008, p. 20). In systems of microorganisms, emerging chirality is found in growing colonies of the bacterium Bacillus subtilis (Ben-Jacob et al., 1995) and of the amoeba Dictyostelium discoideum (Nicol et al., 1999; Levine et al., 2006). Spermatozoa -which are motile and chiral in itself- show the formation of helical paths which are sustained also in the presence of fluctuations (Friedrich and Jülicher, 2009). Another example of active particles (artificial in this case) are rotationally driven spinners which show self-organization into rotating crystals (Nguyen et al., 2014).

\subsubsection{The chiral pattern}

The chiral pattern in our system occurs spontaneously in the nematic region of the phase diagram, i.e. for one set of parameters $\rho$ and $\mathcal{P}$ one simulation can turn out nematic while an independent one (with different initial conditions $\left(\vec{r}_{i} ; \hat{e}_{i}\right)$, different random seeds which influence the vectors $\vec{\xi}_{i}$ ) chiral. A representation of such a chiral pattern is given in Fig. 4.9. The particles within each of the four representative planes (width $\delta \approx 1.1 \epsilon$ ) are ordered nematically which is quantified by a high local nematic 


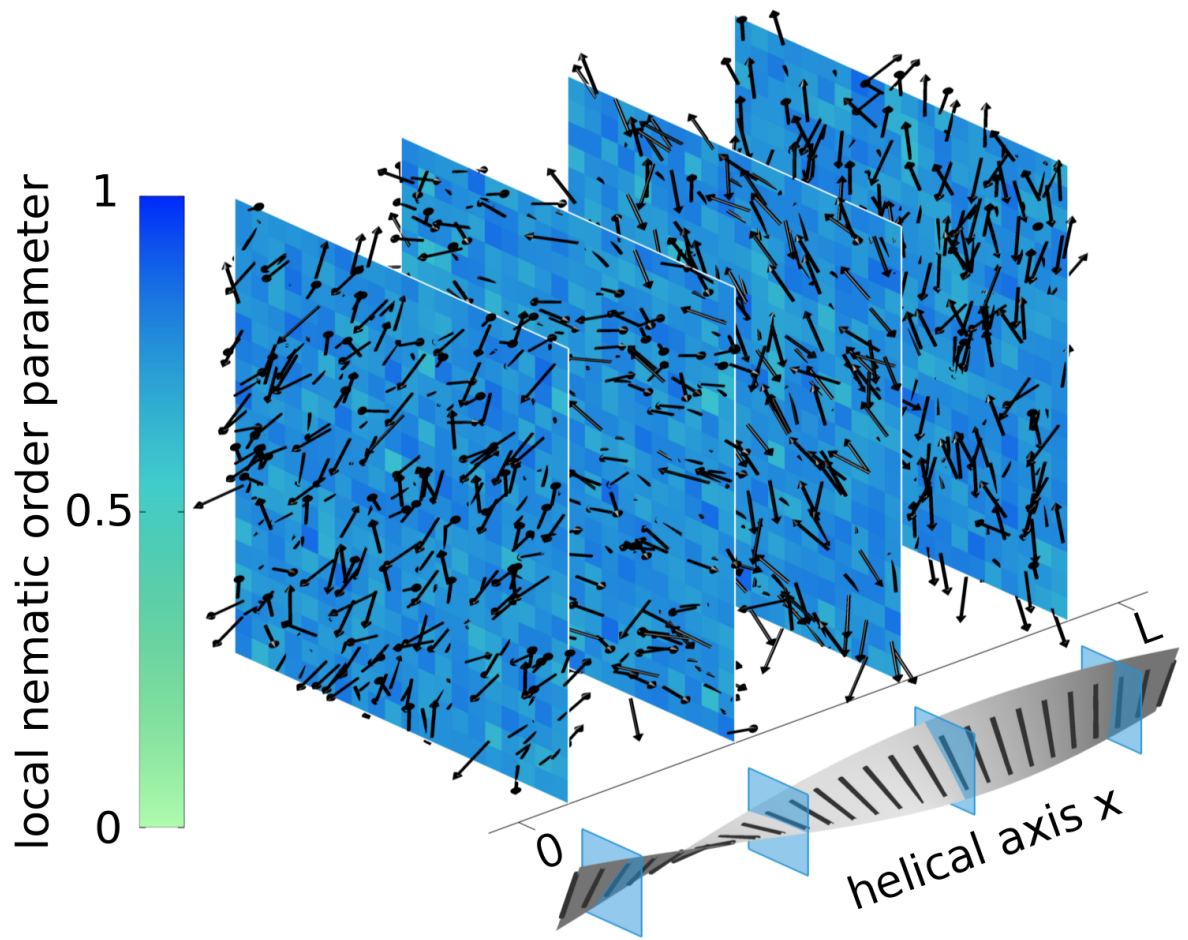

(a) A fraction of the particles (represented by little arrows) are shown in cross section with the corresponding local nematic order parameter (in color). The ribbon below is composed of the local directors in slices perpendicular to the helical axis.

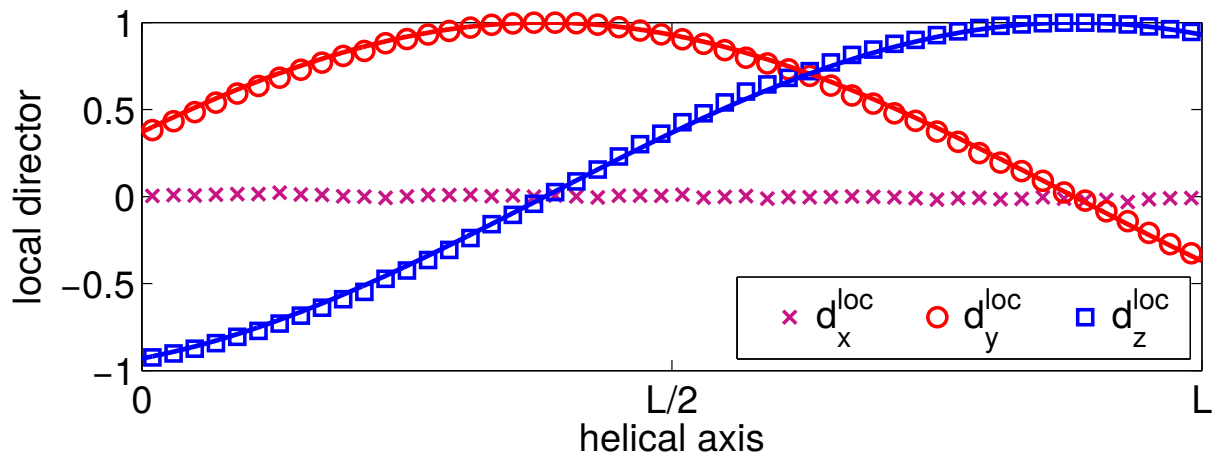

(b) Components of the local director $\hat{d}^{\text {loc }}$ along the helical axis. The symbols denote the measurements while the solid lines are sinusoidal least square fits $d_{y, z}^{\text {loc }}=\cos \left(\pi x / L+\phi_{y, z}\right)$.

Figure 4.9: Snapshot of a chiral pattern $(\rho=1.625, \mathcal{P}=3.29$, reprinted from Breier et al., 2016). 


\begin{tabular}{ccc}
\hline Péclet number & $\begin{array}{c}\text { probability of chiral pattern } \\
P\end{array}$ & $\begin{array}{c}\text { mean absolute } \\
\text { chiral order parameter } \\
\left\langle\left|S_{\chi}\right|\right\rangle_{\chi}\end{array}$ \\
\hline 3.2 & $P\left(\left|S_{\chi}\right|>0.2\right)$ & 0.48 \\
4.0 & $3.3 \%$ & 0.64 \\
5.6 & $5.7 \%$ & 0.74 \\
8.0 & $4.0 \%$ & 0.82 \\
\hline
\end{tabular}

Table 4.2: Results of 300 independent simulations per value of $\mathcal{P}$ at $\rho=1$. The mean absolute chiral order parameter is calculated from all chiral simulations. (Data published in Breier et al., 2016, using a different definition of Péclet number there).

order parameter with a well-defined local director $\hat{d}^{\text {loc }}$. However, as one moves along the helical axis (denoted by $x$ ), this local director rotates around the helical axis which is represented by the ribbon in Fig. 4.9a. The components of the local director (Fig. 4.9b) vary along the helical axis. The two perpendicular components $d_{y}^{\text {loc }}$ and $d_{z}^{\text {loc }}$ are very well fit by sinusoidal functions while the parallel component $d_{x}^{\text {loc }}$ vanishes. This behavior proves that the structure actually is helical.

Each chiral pattern is either right- or left-handed and both enantiomorphs occur with equal probability which substantiates the fact that it is a spontaneous symmetry breaking. Since the simulation has to fulfill the PBCs, only certain directions of the helical axis are allowed just like the possible global directors for the density waves in Eq. (4.22) in three dimensions. Yet, the chiral pattern is mostly aligned along one of the box axes and only rarely along one of the diagonals. We did not observe any other orientations of the helical boxes within the simulation domain. The pitch of the given chiral example is $2 L$ due to the nematic symmetry and the boundary conditions. For differently oriented chiral pattern the pitch would be adjusted according to the orientation of the helical axis.

The probability of the formation of a chiral state as a function of Péclet number is investigated by running 300 independent simulations per value of $\mathcal{P}(\rho=1)$ and counting the number of simulations which in their final state have a chiral order parameter (Eq. 3.73) that is larger than 0.2 in absolute value. This probability (Tab. 4.2) exhibits a maximum at $\mathcal{P}=4.0$. However, the statistics is very limited, so this has to be taken with a grain of salt. The mean absolute chiral order parameter (of the chiral simulations) shows an increase with increasing Péclet number. Thus it behaves similarly to the global nematic order parameter (see Fig. 4.1) which also increases with increasing Péclet number. The reason is that the influence of the stochastic noise decreases with increasing Péclet number thus leading to smaller fluctuations in the orientations which in turn leads to higher global nematic and chiral order parameters. 


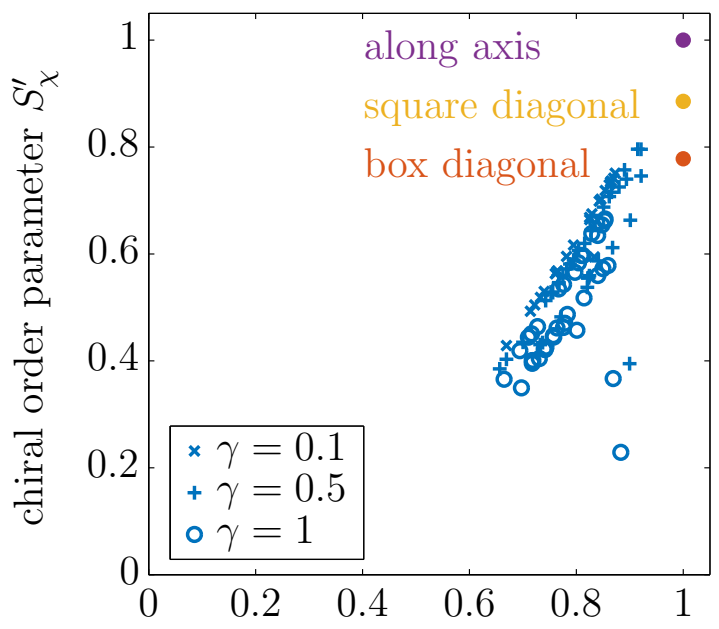

mean local nematic order parameter $\left\langle S_{\text {loc }}\right\rangle$

Figure 4.10: Chiral order parameter versus mean local nematic order parameter of metastable chiral patterns. $S_{\text {loc }}$ is calculated in $20^{3}$ cubic sub-boxes of the simulation box and averaged. The global density $(1 \leq \rho \leq 2)$ and the Péclet number $(2.47 \leq$ $\mathcal{P} \leq 10)$ are varied. The filled dots denote the values corresponding to perfect chiral patterns with different orientations in the simulation box and different pitches (see Section 3.3.2). 
We can substantiate this relationship by comparing the chiral order parameter to the mean local nematic order parameter $\left\langle S_{\text {loc }}\right\rangle$ (see Fig. 4.10). The latter is a measure of the nematic order an achiral pattern with the same system parameters would have. We see that the chiral order parameter increases with $\left\langle S_{\text {loc }}\right\rangle$ just as expected and argued above and it approaches the theoretical value of a perfect chiral for $\left\langle S_{\text {loc }}\right\rangle \rightarrow 1$.

The occurrence of the chiral pattern does not depend only on the initial conditions of a given simulation. In fact, one set of initial conditions $\left(\vec{r}_{i} ; \hat{e}_{i}\right)$ can lead to a nematic, a right- and a left-handed chiral pattern (see Fig. 4.11b). The only difference between the three simulations shown there is a different random seed which leads to different vectors $\hat{\xi}_{i}$. This points to the fluctuations as the key mechanism in the formation of the chiral pattern (see Section 4.4.4 for a detailed discussion). Moreover, we tested the influence of the choice of pseudo-random number generator. For all shown simulations we use the standard C-function drand48(). Simulations with a "Mersenne twister" (Matsumoto and Nishimura, 1998) instead show the same general behavior: longlived metastable chiral pattern occur spontaneously in the nematic region of the phase diagram. The chiral pattern again possesses a homogeneous director twist with a pitch of $2 L$. Hence, the choice of the pseudo-random number generator does not alter the results.

\subsection{3 (Meta-)Stability of the chiral state}

The chiral pattern is long-lived metastable because it can exist at least up to $2.5 \times 10^{6}$ time steps (see examples in Fig. 4.11a). Of all performed simulations only very few show a stable chiral pattern but turn nematic at some point. One such example is investigated in Section 4.4.7. No simulation which possesses global nematic order at one point in time turns chiral. In this sense, the chiral state is a metastable state while the nematic state is stable because the Lebwohl-Lasher potential (Eq. 3.1) is minimized by the nematic configuration. The metastability of the chiral state can be understood from geometric arguments.

The equilibrium nematic director field $\hat{d}$ can be subject to three fundamental deformations as they are shown by De Gennes and Prost (1993, p. 103): splay, bend, and twist. These contribute to the so-called Frank free energy ${ }^{5}$ (or distortion free energy, see De Gennes and Prost, 1993, p. 102)

$$
\begin{aligned}
F & =F_{\text {splay }} \quad+F_{\text {twist }} \quad+F_{\text {bend }} \\
& =\frac{1}{2} K_{1}(\nabla \cdot \hat{d})^{2}+\frac{1}{2} K_{2}(\hat{d} \cdot(\nabla \times \hat{d}))^{2}+\frac{1}{2} K_{3}(\hat{d} \times(\nabla \times \hat{d}))^{2}
\end{aligned}
$$

with the elastic constants $K_{1}, K_{2}$, and $K_{3}$. A schematic representation of these three

\footnotetext{
${ }^{5}$ given per volume
} 

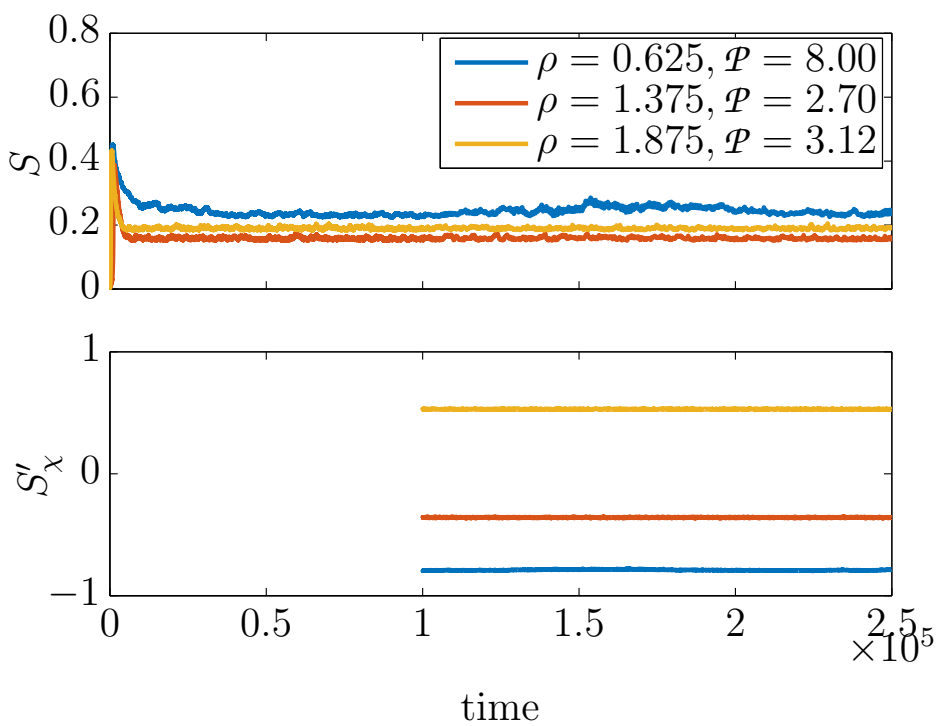

(a) Three long simulations which show the metastability of the chiral pattern. Local density and Péclet number are used as indicated. The global nematic and chiral order parameters are stable for at least $2.5 \times 10^{6}$ time steps $(\Delta t=$ $10^{-1}$ ) after the start of the simulations. The calculation of the chiral order parameter started after time $=10^{5}$.

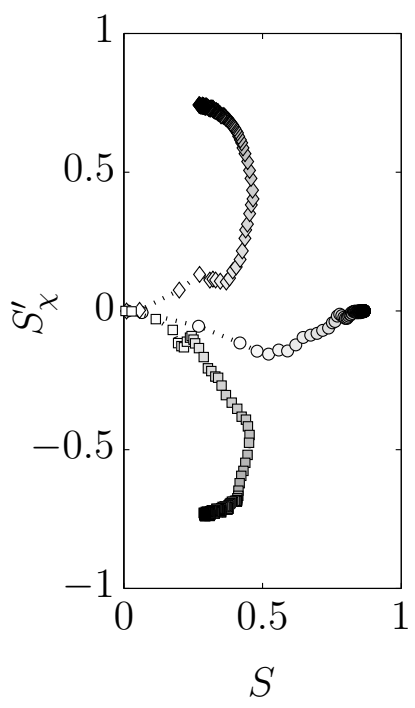

(b) Three simulations which start from the same initial conditions $\left(\vec{r}_{i}, \hat{e}_{i}\right)$ with different random seeds. Time increases from white to black.

Figure 4.11: Investigation of different properties of the chiral pattern: Long-lived metastability and spontaneous symmetry breaking.

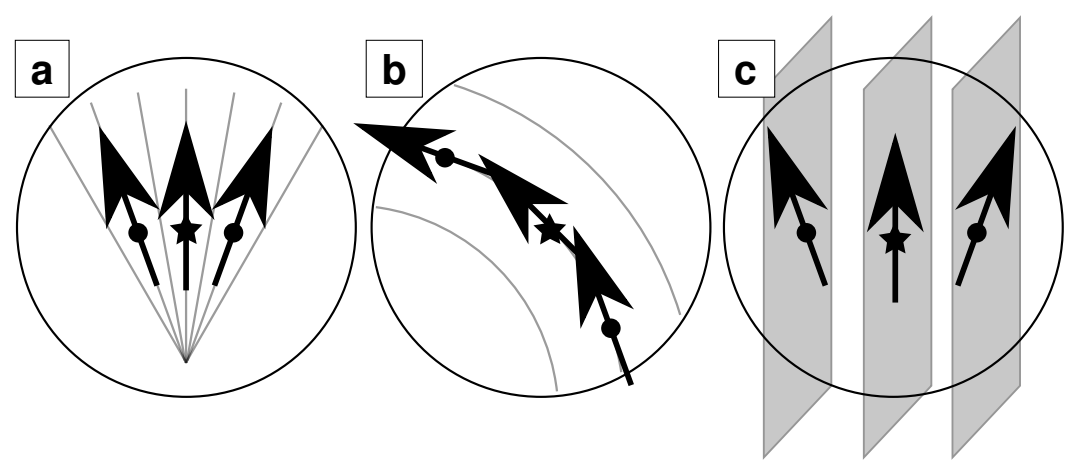

Figure 4.12: Sketches of the three fundamental deformations of a nematic director field: Splay (a), bend (b), and twist (c). The reference particle ( $\star$ ) interacts with its neighbors within the interaction range (circle). No torque is excerted on it by the two neighboring particles $(\bullet$, picture reprinted from Breier et al., 2016). 
deformations is given in Fig. 4.12. The symmetry of all three ideal deformations is such that a given reference particle does not experience any torque. For any neighboring particle which would exert a torque on the reference particle, there exists a second neighboring particle which leads to an equal and opposite torque. Hence, the total torque on the particle vanishes. However, in our system of self-propelled particles only the chiral deformation can persist. The self-propelled particles cannot form a splay deformation because there exist no sinks or sources in the system. Due to the lack of a centrifugal force, the bend deformation is not stable because the particles cannot be forced onto a curved path. Finally, the twist deformation is stable in principle because each particle moves within a nematically ordered slice.

\subsubsection{Formation of the chiral state}

A twist deformation is stable in our system, but how does it form? We will answer this question in the following and also elaborate on the role of fluctuations in the system by comparing to a system of non-motile particles.

All simulations start from a homogeneous and isotropic distribution of particles' positions and orientations. The temporal evolutions of the global order parameters for a system that develops into a chiral state (Fig. 4.13) show that in the initial phase (here up to $t \approx 280$ ) the nematic order parameter is larger than the chiral order parameter. At the final stage, the chiral order parameter is roughly two and a half times larger than the nematic order parameter. Interestingly, the smectic order parameter is comparable to the nematic order parameter and the polarization might still increase. The temporal evolution in $\left(S, S_{\chi}\right)$-space substantiates this. The order parameters only tell us something about the overall structure in the system. Any structure in principle emerges from local interactions among the particles and then spreads out to span eventually the whole system, hence, what we need to measure is a local quantity and see how it develops. Since we know the final orientation of the helical axis, we can study the system in slices perpendicular to that axis. The mean square orientation $\left\langle e_{x, y, z}^{2}\right\rangle$ within these planes is given in Fig. 4.13b. We observe the persistence of a roughly isotropic distribution until $t \approx 150$ and then the chiral structure emerges. From this analysis, it is hard to judge what exactly triggers the onset of the chiral pattern. We can only observe the onset of chirality in a clear-cut fashion at $t \approx 200$. One additional drawback of this method is that we already impose the helical axis from the beginning and cannot exclude a bias by investigating slices from the very beginning.

To understand this problem, we now want to examine a local quantity from which we can learn how the chiral pattern emerges from the local nematic alignment. We 

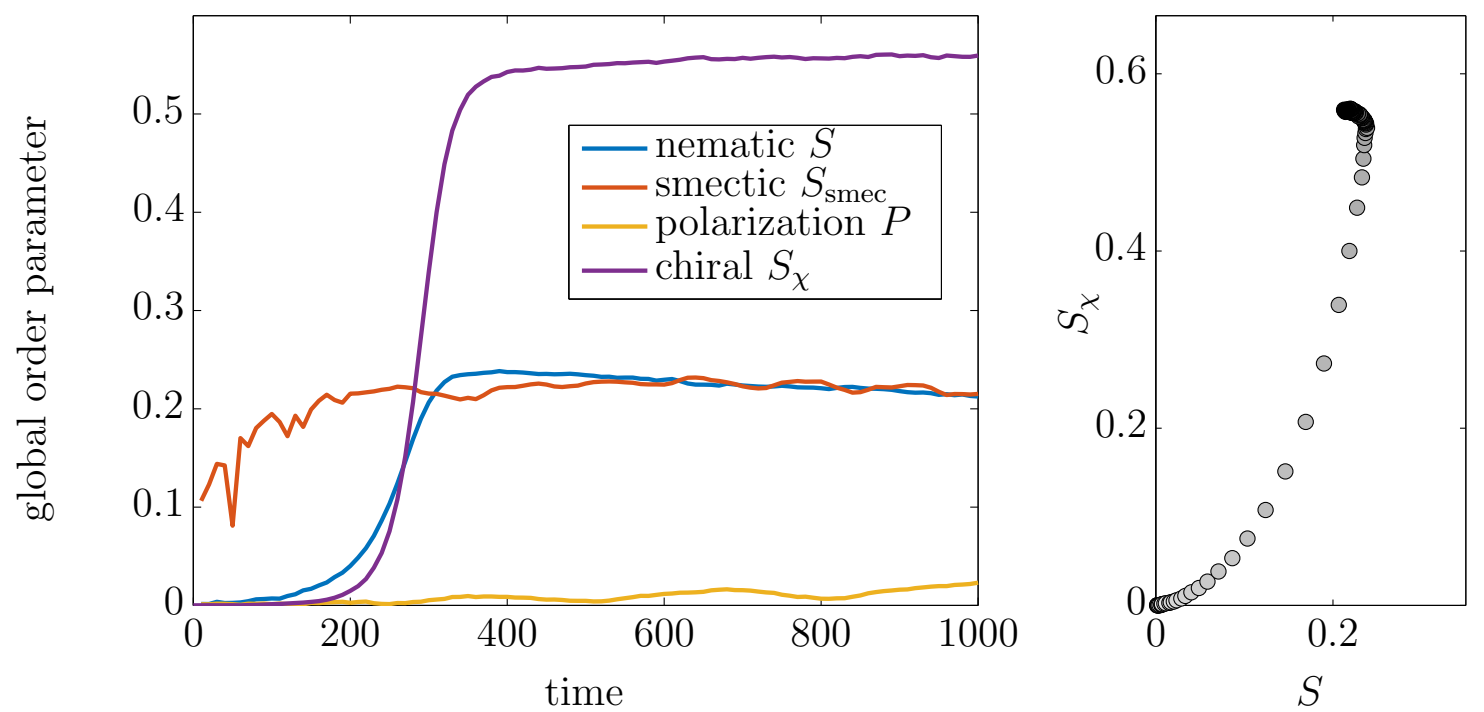

(a) Temporal evolution of the global order parameters. The right panel shows the evolution in $\left(S, S_{\chi}\right)$-space with time increasing from white to black (the temporal evolution of the chiral order parameter was published in Breier et al., 2016).
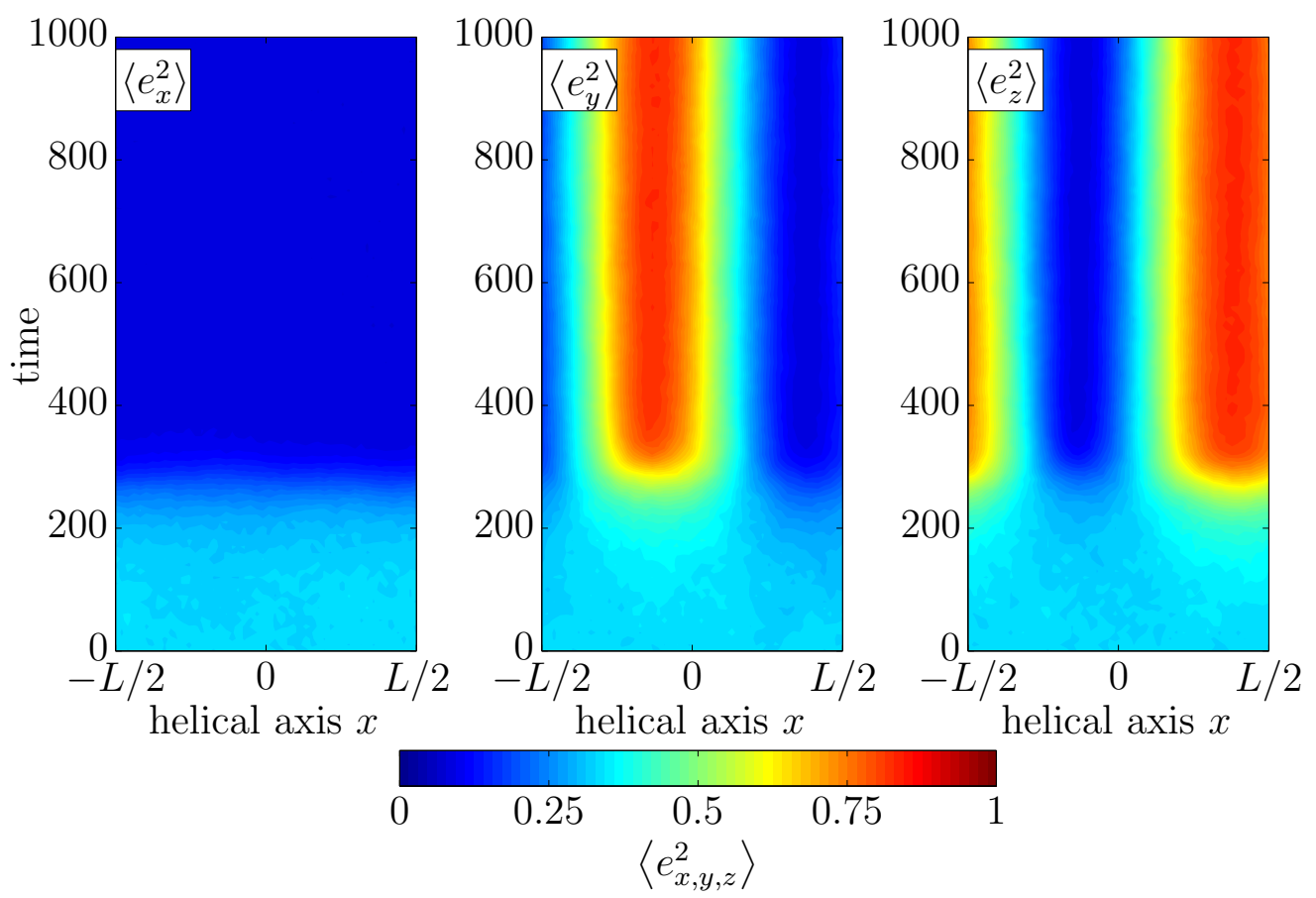

helical axis $x$

(b) Mean square components of the orientations of the particles. The system is divided into 50 slices perpendicular to the helical axis $x$ and the mean square of each orientation component is calculated. 100 snapshots are evaluated in total.

Figure 4.13: Temporal evolution of the chiral simulation in Fig. 4.9 ( $\rho=1.625$, $\mathcal{P}=3.29)$. 


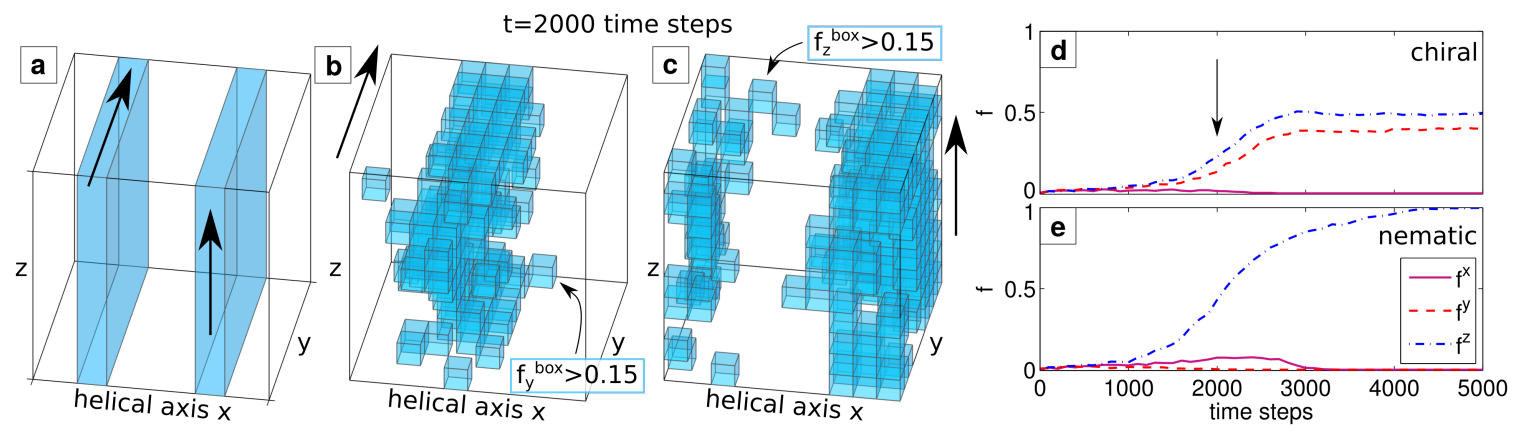

Figure 4.14: Emergence of the chiral pattern (cf. Figs. 4.9 and 4.13). (a) Sketch of two nematically order layers with an angle of $\pi / 2$ between their local directors. (b) and (c) After 2000 time steps, the system is divided into $10^{3}$ boxes and those with $f_{\text {box }}^{y}>0.15$ and $f_{\text {box }}^{z}>0.15$ are plotted (see Eq. 4.25 for definition). (d) and (e) Temporal evolution of the fraction of boxes mostly aligned along one axis (see Eq. 4.26) for the chiral pattern and a nematic pattern for comparison. Reprinted figure (with additional annotations) with permission from Breier et al., Physical Review E, 93(2):022410, 2016. Copyright 2016 by the American Physical Society.

divide the system into $10^{3}$ boxes and measure in each box

$$
f_{\text {box }}^{\{x, y, z\}} \equiv \frac{\#\left(\text { particles with }\left|e_{x, y, z}\right|>0.9\right)}{\#(\text { particles in box })}
$$

which is the fraction of particles in the box that are mostly aligned along one of the axes. We examine the evolution of the chiral pattern in terms of this measure. After 2000 time steps $(t=200$ in Fig. 4.13$)$ the nematic order parameter is larger than the chiral order parameter but the latter is larger than zero. Figure 4.14 (panels b and c) shows the small boxes inside the simulation domain where after 2000 time steps $f_{\text {box }}^{y}>0.15$ and $f_{\text {box }}^{z}>0.15$, respectively. We can clearly identify two distinct layerlike areas perpendicular to the final helical axis which show local nematic alignment with an angle of roughly $\pi / 2$ between the two local directors. These two layers (see also Fig. 4.14, panel a for a sketch) are very typical during the formation of the chiral pattern. The particles firstly interact locally and align nematically hence forming small nematic domains. These domains then start competing and can either lead to a nematic or a chiral pattern. The fraction of boxes with particles which are mostly aligned along one of the axes is given by

$$
f^{\{x, y, z\}} \equiv \frac{\#\left(\text { boxes with } f_{\text {box }}^{\{x, y\}}>0.15\right)}{\# \text { boxes }}
$$

and can be tracked over time (Fig. 4.14, panels $\mathrm{d}$ and e). In the beginning all three 
quantities $f^{x}, f^{y}$, and $f^{z}$ show a slight increase for both the nematic and the chiral pattern. For the nematic pattern we then see that $f^{z}$ finally dominates; the global nematic director was chosen spontaneously to be parallel to $\hat{z}$. In the case of the chiral pattern, the $f^{y}$ and $f^{z}$ are finally similarly important while $f^{x}$ decays.

To strengthen this argument we can perform the following numerical experiment: We seed the system with nematically ordered planes instead of isotropic initial conditions and measure the probability that a chiral pattern forms. Initially, we place the particles at random positions into the simulation box. Two slices of the simulation box are chosen to form the nematically ordered planes. Their individual width is $0.05 L$ and they are a distance of $L / 2$ apart from each other. All particles within each of these slices have the same initial orientation (in the nematic sense), while the two local directors of the two slices enclose an angle of $\pi / 2$. The system is then evolved until a steady-state is reached. Sixty independent such simulations yield an increase of the chiral probability to about $50 \%$. Thus, the two nematically ordered planes are a precursor of the chiral state even though fluctuations are important since the formation of a chiral state is not completely determined by the nematically ordered planes.

\subsubsection{Comparison to one-dimensional model}

We see that fluctuations play a crucial role in the formation of the chiral pattern because the same initial state can lead to a chiral or nematic state depending on the fluctuations. Moreover, seeding the system with the precursor of a chiral pattern (two parallel, nematically ordered planes whose local directors form an angle of about $\pi / 2$ ) does not always lead to a chiral pattern but only increases the probability. Hence, the fluctuations are still of high importance. Moreover, as we will see in Section 4.4.7, a chiral pattern can untwist and form a nematic pattern only by the appropriate fluctuations. In this section we will elucidate the role of the fluctuations by examining a one-dimensional system of non-active interacting spins (simulations performed by R. Selinger, Kent State University, USA, published in Breier et al., 2016) similar to the classical $X Y$-model. The $N_{r}$ spins (or rotors) are placed next to each other (see inset of Fig. 4.15) on a line with PBCs. Each spin can rotate around the axis which connects all spins and hence is described by its rotation angle $\theta_{i}$. The spins interact (just like the SPPs) via the Lebwohl-Lasher potential (Eq. 3.1) which for this model can be rewritten as

$$
V=-\sum_{i=1}^{N_{r}} J \cos \left[2\left(\theta_{i+1}-\theta_{i}\right)\right]
$$

with the coupling constant $J$. This potential is very similar to the potential of the classical $X Y$-model except for the additional factor of two which leads to nematic 


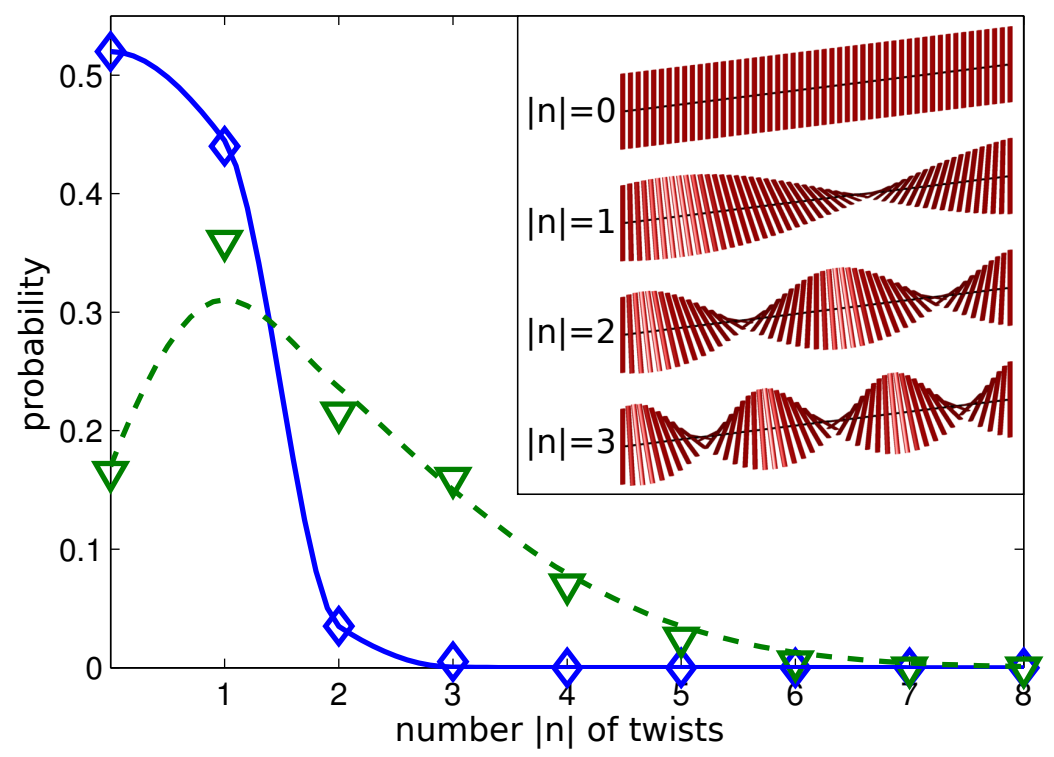

Figure 4.15: Results of the one-dimensional Lebwohl-Lasher model. Inset: It can evolve to twisted metastable states with $\pm n$ twists or the untwisted ground state. Main panel: Probability of the number of twists for a long chain with $N_{r}=800$ (green $\nabla$ ) and for a short chain with $N_{r}=100$ (blue $\diamond$ ). The lines are guides for the eye. Reprinted figure with permission from Breier et al., Physical Review E, 93(2):022410, 2016. Copyright 2016 by the American Physical Society. 
instead of polar symmetry. The system is described by the Hamiltonian

$$
H=V+\sum_{i=1}^{N_{r}} \frac{1}{2} I \omega_{i}^{2},
$$

with the moment of inertia $I$ (which can be set to unity) and the angular velocity $\omega_{i}$ of the $i$-th rotor. The torque of the $i$-th rotor is given by

$$
\tau_{i}=-I \frac{\partial V}{\partial \theta_{i}}
$$

such that the rotor is influenced by its two neighbors. The angular acceleration $\alpha_{i}$ is then proportional to the torque

$$
\alpha_{i}=\frac{\tau_{i}}{I}
$$

and needs to be integrated forward in time to obtain the angular velocity $\omega_{i}$. The temporal integration of the latter then leads to the angular position of the rotor (i.e. the angle $\theta_{i}$ ). This integration in time is done using a velocity Verlet algorithm and a nonequilibrium rapid quench (Langevin thermostat) from $T=10$ to $T=10^{-7}$ in $6 \times 10^{5}$ time steps which successively removes kinetic energy from the system. The initial configuration consists of random directions of the rotors and zero angular velocities. For each chain length 200 such annealing trials are carried out independently. We find that for long chains $\left(N_{r} \geq 200\right)$ the most likely final state is a chiral state with a twist of $\pm \pi$ while the nematically ordered state is the most probable for shorter chains (see Fig. 4.15). This behavior can be inferred from the associated energies. The energy of the nematic ground state is $E_{0}=-J$. Possible chiral states show a rotation angle of $n \pi$ along the chain due to the PBCs and the nematic interaction. The potential energy of such a chiral state is given by

$$
\Delta E_{n}=\langle-J \cos (2 \Delta \theta)\rangle-E_{0}
$$

where the subscript $n$ refers to the number of half-twists. The angle between neighboring rotors (for a homogeneous twist) is given by

$$
\Delta \theta=\frac{n \pi}{N_{r}} .
$$

For long enough chains, we can make use of the small-angle approximation and find

$$
\begin{aligned}
\Delta E_{n} & \approx\left\langle-J\left(1-2 \Delta \theta^{2}\right)\right\rangle-E_{0} \\
& =2 J\left(\frac{n \pi}{N_{r}}\right)^{2} .
\end{aligned}
$$


This means that there are considerable energy barriers between the different states and if a system is quenched from a high temperature, random state to a low temperature one it is possible that it moves (in the energy landscape) into a twisted state instead of the untwisted ground state. Moreover, the height $\Delta E_{n}$ of the energy barrier decreases with increasing chain length. The fugacity of the corresponding equilibrium model $\exp \left(-\Delta E_{n} /\left(k_{B} T\right)\right)$ forms a Gaussian bell-curve as a function of the number of twists $n$ and hence always exhibits a maximum at $n=0$. However, if we do not distinguish left- and right-handed helices and study the fugacity as a function of $|n|$, we find (for long enough chains) a maximum at $|n|=1$. This means that the twisted state is more probable than the untwisted ground state if the chain is long enough. Moreover, if we increase the chain length further, chiral states with more twists become more likely. In the limit of an infinitely long chain this destroys the long-range order in the system at any finite temperature and leads to the phase transition at $T=0$ (just like in the $X Y$-model). In Fig. 4.15 we plot the probability of the states with different numbers $|n|$ of twists and compare systems with different chain length. We find that in a short chain $\left(N_{r}=100\right)$ the nematic state is the most probable. However, for a long chain $\left(N_{r}=800\right)$ the chiral state with a twist of $|n|=\pi$ occurs more often than the nematic ground state just as predicted. If we used instead of $|n|$ the number $n$ of twists (distinguishing between left- and right-handed helices), the curve would always be bell-shaped with a maximum at $n=0$. However, for larger chain lengths twisted states are more likely than for shorter chain and the distribution along $n$ gets broader, so that the combined probability of $n=+1$ and $n=-1$ is larger than the probability of $n=0$ for a chain that is long enough (i.e. $N_{r} \geq 200$ ). An additional finding of this one-dimensional model is that the mean square number of twists $\left\langle n^{2}\right\rangle$ increases linearly with the number of rotor $N_{r}$ and hence follows the same statistics as a random walk.

An equivalent effect can also be found in the system of SPPs: The probability of a chiral state is more than ten times larger in a long box of aspect ratio $10: 1: 1$ $(N=230 \times 23 \times 23, \rho=1, \mathcal{P}=3.13)$ than in a cubic box. Moreover, not all chiral patterns in such a box show one half twist inside the simulation box but also the spontaneous formation of a fully twisted chiral (pitch of $L_{x}$ ) can be observed. However, it is important to note that such a long box requires longer relaxation times until a true steady-state is reached. The fully twisted chiral is hence also expected to be metastable just like the chiral state in general in the cubic simulation domain. We also find metastable configurations where the local nematic director is oriented perpendicular to the long axis of the simulation box. If one moves along this axis, the orientation of the local nematic director undulates but does not lead to a helix. Such a state is only possible due to the long time it takes until information is propagated along the long axis of the simulation domain. 


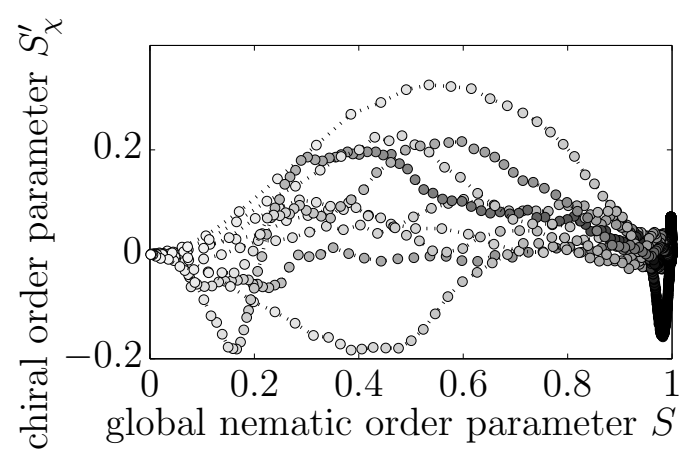

(a) Example trajectories (time increases from white to black) in nematic and chiral order parameter $\left(N=66^{3}\right)$.

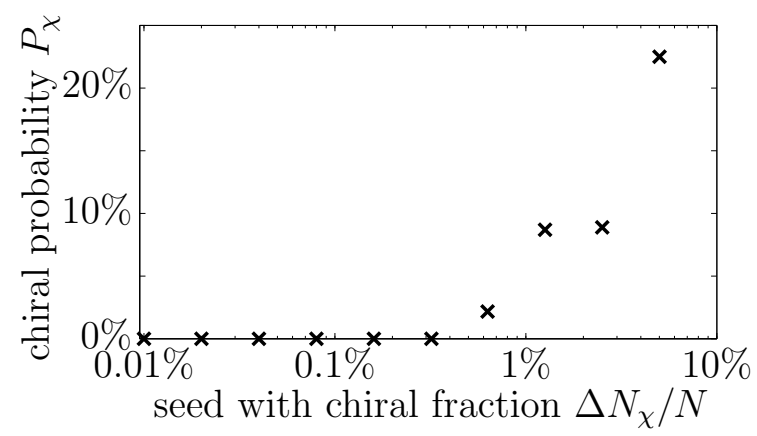

(b) Probability of a persistent chiral pattern when the box is seeded with a fraction of $N_{\chi} / N$ particles which follow a chiral pattern $\left(N=50^{3}\right)$. For each data point 50 independent simulations are performed.

Figure 4.16: Simulations of SPPs with $\mathcal{P} \rightarrow \infty(\rho=1, \gamma=0.1)$.

\subsubsection{Importance of fluctuations}

All simulations up to this point are performed with a finite Péclet number and spontaneous chiral symmetry breaking is observed everywhere in the phase space which corresponds to the nematic phase. We also know from the one-dimensional, non-selfpropelled model that orientational fluctuations are very important because they may trap the system in a twisted state and prevent reaching the untwisted ground state. In our model of SPPs, we can also study the influence of fluctuations by simulating the deterministic model with $\mathcal{P} \rightarrow \infty$. The resulting trajectories in the $\left(S, S_{\chi}\right)$-plane (see Fig. 4.16a) have a very similar appearance to the ones corresponding to simulations with finite Péclet number (see Fig. 4.13a). In the initial phase both $S$ and $S_{\chi}$ grow similarly. However, all trajectories evolve to the nematically ordered state $(S \rightarrow 1$, $\left.S_{\chi}=0\right)$. A study of 300 independent simulations with vanishing noise reveals that all of them evolve to a nematic state. This indicates that the fluctuations are crucial for the formation of a chiral pattern. The initial increase in $S_{\chi}$ in the temporal evolution can be ascribed to the formation of parallel, nematically ordered layers with nonparallel local directors just like in the case of finite $\mathcal{P}$. However, rotational diffusion is necessary for the formation of a chiral pattern. The microscopic interactions between particles lead to nematic alignment but rotational diffusion counteracts this mechanism in the sense that the alignment can never be perfect because orientations are altered by rotational diffusion. In a deterministic system without rotational diffusion $(\mathscr{P} \rightarrow \infty)$ and above the critical Péclet number only the nematic state can form from an isotropic configuration. The reason is that the alignment between neighboring particles will eventually be perfect and result in global nematic alignment. Instead 
in the case of a finite Péclet number the chiral state can form as well. In this state neighboring particles along the helical axis are not aligned but their orientations form a finite angle. The imperfect alignment due to rotational diffusion can favor such a configuration during the transient which allows for the formation of a chiral state in the presence of rotational diffusion. This finding matches also the decreasing chiral probability for increasing Péclet number (see Tab. 4.2).

Even though the deterministic system does not form spontaneously a chiral pattern, we can seed the system with such a configuration and investigate whether it is stable over time. We initialize such a simulation by placing the particles randomly but homogeneously into the simulation domain. Then we randomly choose a fraction $\Delta N_{\chi} / N$ of the particles and assign to them the orientation vector according to a perfect chiral pattern with a pitch of $2 L(\hat{e}=(0 ; \cos (\pi x / L) ; \sin (\pi x / L))$. All other particles are assigned a random orientation on the surface of the unit sphere, just like in the isotropic initialization. The result of 50 such independent simulations per value of $\Delta N_{\chi} / N$ (see Fig. 4.16b) reveal that a chiral fraction of $\Delta N_{\chi} / N \approx 0.6 \%$ is enough to obtain a small yet finite probability for a persistent chiral pattern. This chiral probability increases as the chiral fraction increases. In the limiting case of $\Delta N_{\chi} / N=1$, the chiral probability is expected to reach unity. We know that the chiral pattern (in case of a homogeneous particle distribution) is stable (see Section 4.4.3). For a deterministic simulation there is hence no possibility to untwist a perfect, homogeneous chiral pattern.

To summarize, we have found that the fluctuations are crucial for the formation of a chiral pattern but the chiral pattern can also be stable without stochastic noise.

\subsubsection{An untwisting chiral pattern}

The chiral state is long-lived metastable as we have seen before since it can last for up to $2.5 \times 10^{6}$ time steps. In all simulations which were found to be chiral, there have been very few examples of a chiral state which show the metastability by existing for a considerably long time followed by untwisting and the formation of a nematic state. One such example is reported in Fig. 4.17. The chiral order parameter is given in absolute value since the handedness of the helix is not of particular interest in this case. The system starts from an isotropic random configuration and the chiral pattern is formed at $t=5000$. The chiral and nematic order parameters appear to be rather constant until $t=20000$ but the polarization increases over time with a superimposed apparent oscillation. The corresponding snapshot at $t=20000$ shows a well-developed chiral pattern. The transition then takes about 20000 time units where $\left|S_{\chi}\right|$ decreases and both $S$ and $P$ increase (with fluctuations/oscillations). When the chiral order parameter reaches zero both the nematic order parameter and the polarization show a very abrupt increase. The snapshot at the transition $(t=35000)$ shows that the global director is not perpendicular to the former helical axis but they form an angle. 


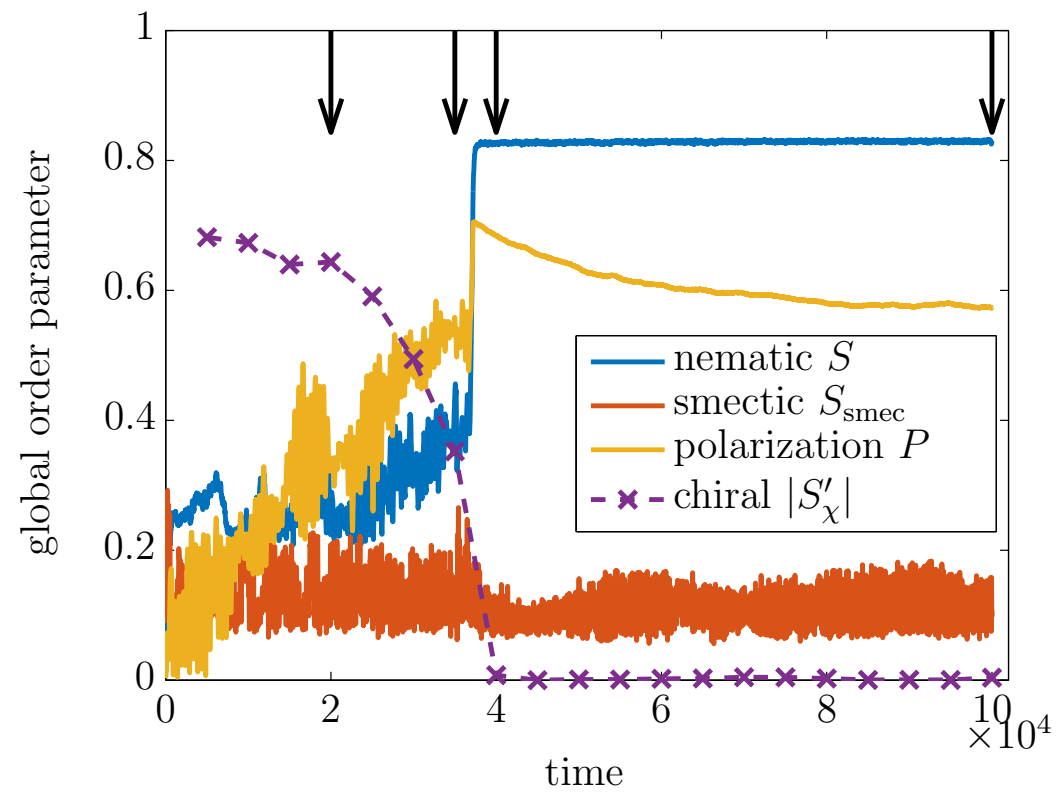

(a) Global order parameters over time. The black arrows indicate the points in time for which the snapshots are given.

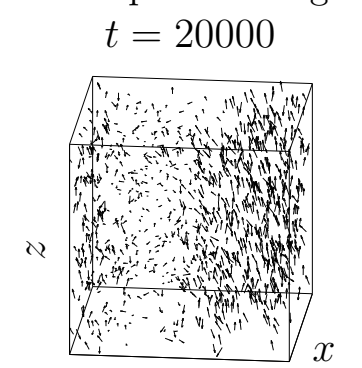

$y$

$$
t=35000
$$

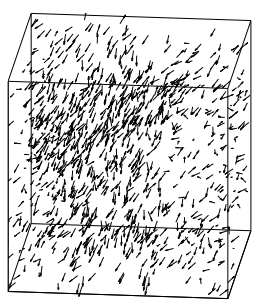

(b) Corresponding snapshots.
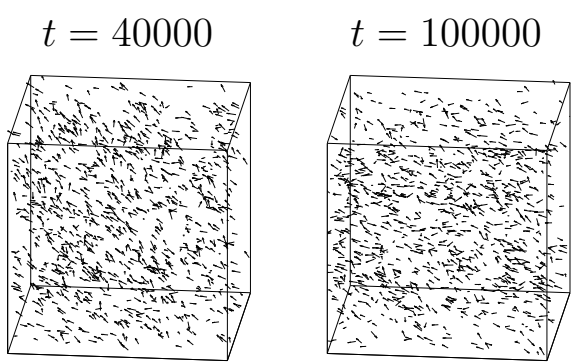

Figure 4.17: Evolution of a system from an isotropic state via a chiral state to a nematic state $(\rho=1.625, \mathcal{P}=6.17)$. 
From that point on the nematic order parameter remains constant at a high level, the chiral order parameter vanishes, and the polarization decreases from about 0.7 to below 0.6. The steady-state configurations show a nematically ordered, homogeneous system. The smectic order parameter is fluctuating around a low value throughout the whole simulation.

It is important to note that the transition from a chiral configuration to a nematically ordered system is a rare event. Moreover, we find from the temporal evolution of the order parameters that a possible precursor of this event might be the increasing and oscillating global polarization: in the initial phase (up to $t=20000$ ), all global order parameters appear roughly constant while the polarization grows from zero to about 0.4 with strong oscillations. This also indicates that the given chiral pattern is not fully in steady state.

In the following section, we elucidate the role of these oscillations and can eventually conclude how these might help in untwisting a chiral pattern.

\subsubsection{Oscillations in the polarization - A density wave?}

The simulations which exhibit chiral symmetry breaking are not only identified by a high chiral order parameter together with a low global nematic order parameter (as compared to other simulations in the same region of the phase diagram), but also typically show very regular oscillations in the polarization about a mean value which indicates a small but finite degree of global polarization (typically $0 \leq\langle P\rangle \leq 0.2$ ). In this section, we will investigate one particular example of a chiral pattern which shows an oscillating global polarization.

Figure $4.18 \mathrm{~b}$ shows the temporal evolution of the global order parameters after the transient has died out. A rather constant chiral order parameter and very regular oscillations in both the polarization and the nematic order parameter are visible. Both $S$ and $\mathcal{P}$ fluctuate around a similar mean value of about 0.25 but the amplitude of the polarization is much larger than that of the nematic order parameter. They seem to be in phase for the first three to four periods and then the frequency of the nematic order parameter appears to decrease compared to the one of the polarization. We are not only interested in the magnitude of the polarization but also in its direction. To this end, we measure the angles between the polarization vector $\hat{P}$ and the helical axis $\hat{x}^{6}$. The azimuthal angle in a plane perpendicular to the helical axis is denoted by $\phi$ and the angle of elevation with respect to that plane is $\theta$ (see Fig. 4.18a). Both angles show a periodic pattern with the same frequency as the polarization order parameter. The elevation angle is always negative and shows an oscillation. Its minima coincide with the minimal values in $P$. However, the azimuthal angle does not oscillate but it

\footnotetext{
${ }^{6}$ We find the helical axis by visual inspection of the snapshot, it is mostly given by one of the axes of the simulation domain.
} 


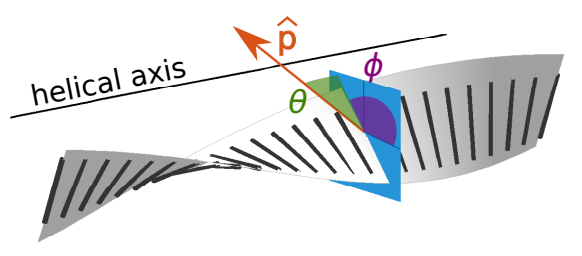

(a) The global polarization $\hat{p}$ of the chiral pattern can be described by two angles with respect to the helical axis: $\phi$ is the azimuthal angle in the plane perpendicular to the helical axis. $\theta$ is the elevation with respect to this plane. The helical axis of a given simulation is found by inspection of the corresponding snapshot.
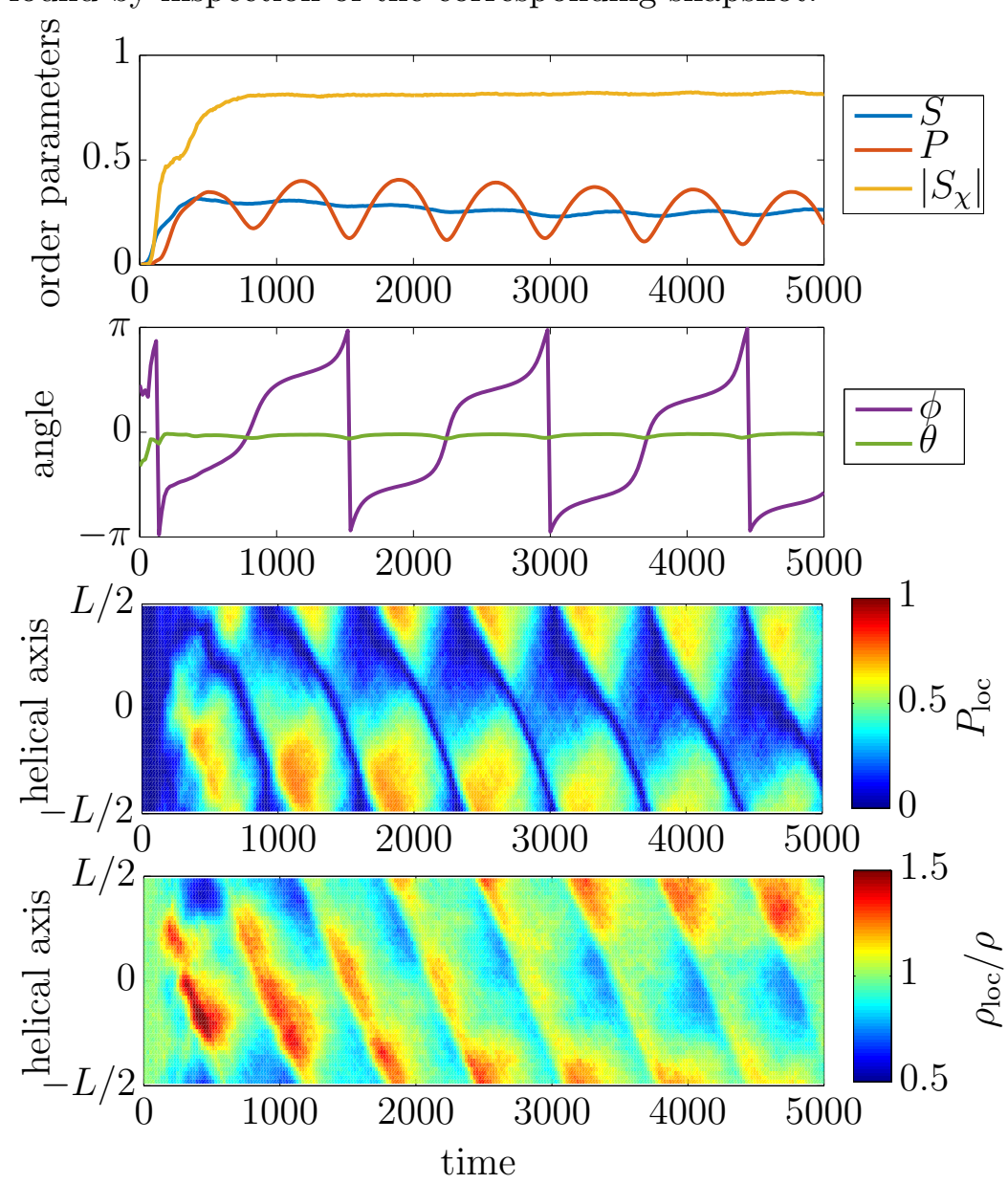

(b) Temporal evolution of the order parameters (measured every 10 time units), of the angles between polarization and helical axis (measured every 20 time units), and of the local densities and local polarization (measured every 20 time units in 50 slices perpendicular to the helical axis).

Figure 4.18: Oscillations in polarization in a chiral simulation $(\rho=1, \mathcal{P}=8)$. 
increases over time with jumps from $+\pi$ to $-\pi$. The slope of $\phi(t)$ is not constant, it exhibits a maximum where $\theta$ reaches its minimum. To sum up, the overall periodic movement of the polarization direction goes as follows: The vector $\hat{p}$ rotates around the helical axis with an additional (small) movement out of the plane perpendicular to the helical axis. The polarization vector lies in the plane, when the azimuthal angle is approximately $\pm \pi / 2$, i.e. $\hat{p}$ is parallel to $\hat{y}$ (and at the same time $P$ is maximal). On the other hand, $|\theta|$ reaches its maximum where $\phi$ is approximately $\pm \pi$ and hence $\hat{p}$ is never parallel to $\hat{z}$ but it points maximally out of the $y-z$ plane when it rotates over the $z$-axis. The described rotation of $\hat{p}$ is clearly visible in Fig. 4.19a.

The global polarization vector does not reveal fully what happens in the system since the value of the polarization is, though significantly higher than in a nonpolarized system, lower than what one would expect for a polarized system. Hence, we need a local information, i.e. local polarization and local density. Both are measured in 50 equidistant slices perpendicular to the helical axis (see two lowermost panels in Fig. 4.18b), since we expect from the changes in direction of the polarization vector that the layers perpendicular to the helical axis play an important role.

The spatio-temporally resolved pattern in $P_{\text {loc }}$ and $\rho_{\text {loc }}$ reveal the following: At any instant in time, the local polarization is not constant over the helical axis but it typically exhibits one maximum and one minimum. However, there are time instants where it roughly vanishes everywhere which coincide with the minima of the global polarization $P$. The normalized local density, instead, shows a maximum at all times which moves at a roughly constant speed along the helical axis (tilted yellow lines in the $\rho_{\text {loc }}(x, t)$ plot). In addition the maximum strengthens and weakens over time (occurrence of red spots in the plot). These spatio-temporal areas of high local density concur with the maxima in the local polarization. This points to a density wave which moves along the helical axis and is coupled to the local polarization.

The speed of this wave-like structure can be inferred by finding the maximum of local polarization or local density at any time instant (see Fig. 4.20a). The movement of the position of this maximum (denoted by $x\left(P_{\max }\right)$ and $x\left(\rho_{\max }\right)$ ) over time allows us to estimate the speed (see Fig. 4.20b). The position of the maximum in local density shows a constant speed in rather large time windows while the maximum in local polarization moves constantly over shorter time periods and then shows jumps. We choose two (one) time windows for $x\left(P_{\max }\right)$ (for $x\left(\rho_{\max }\right)$ ) where we perform linear least-square fits. The resulting speeds are $v_{\rho}=0.10$ for the local density and $v_{P, 1}=$ 0.063 and $v_{P, 2}=0.087$ for the local polarization. We find that the density wave moves at a speed of roughly one fifth of the self-propulsion speed of the particles $\left(v_{0}=0.5\right)$. The maximum in local polarization moves a little slower, though.

The remaining question is whether the helix of local directors rotates in time or is static. To investigate this, we compare the profiles along the helical axis over one period of the global polarization (see Fig. 4.19b). The profile of one component of the local nematic director (in this case $d_{\mathrm{loc}, y}$ ) shows only small changes over the course 

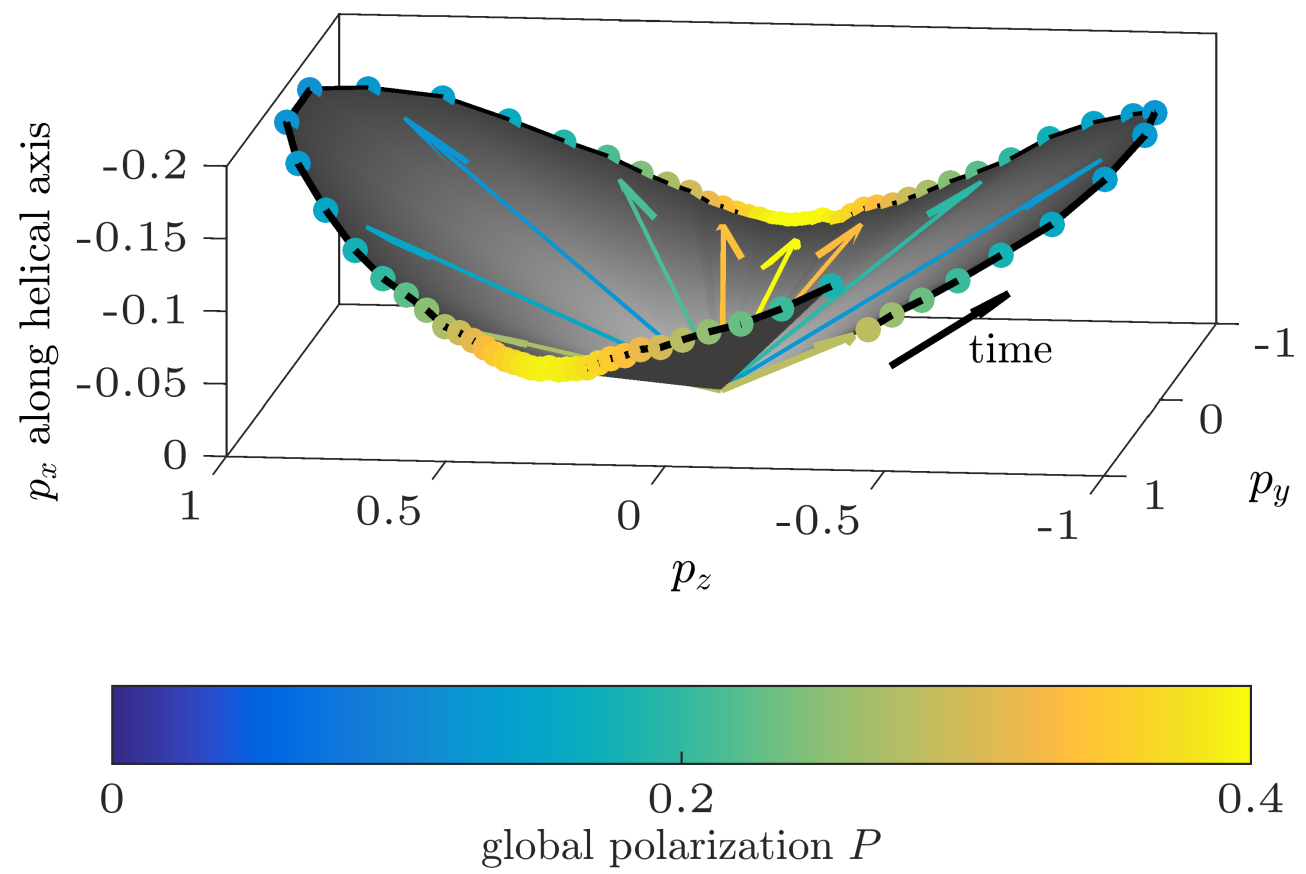

(a) Rotation of the global polarization vector in a chiral pattern with oscillating global polarization (time $t \geq 1000$ ). The dots denote the different directions of $\hat{p}$ on the surface of the cone with $P$ as color and a few orientations of $\hat{p}$ are shown in an exemplary way. The trajectory of $\hat{p}$ is not closed which leads to an open cone. The vertical axis is stretched for the sake of clarity.

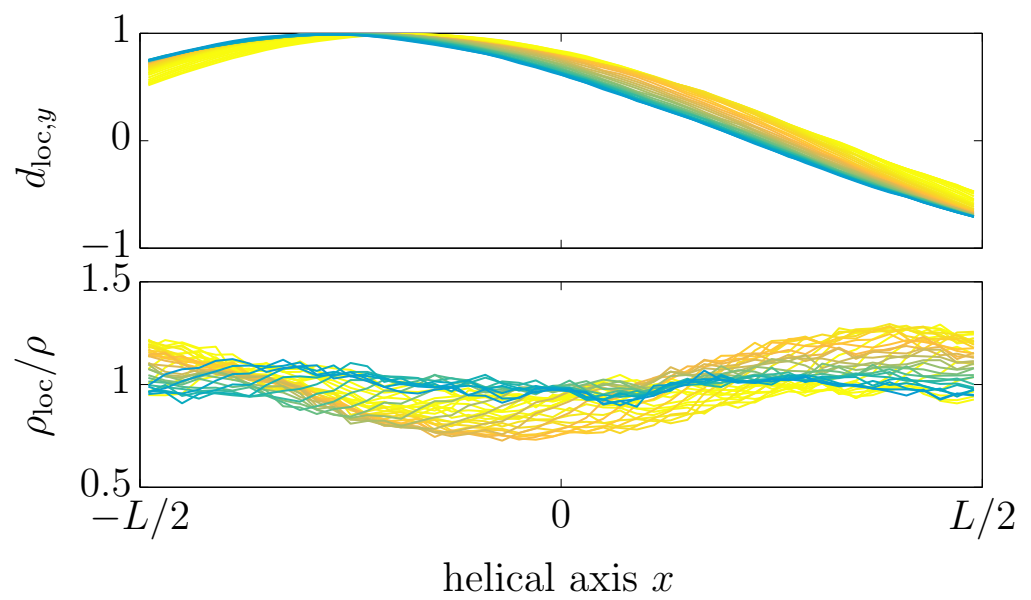

(b) Profiles of local density and one component of the local director during one period of the oscillation of $P$ (time $3690 \leq t \leq 4400$ ). The color is chosen according to the global polarization (see left panel).

Figure 4.19: Further investigation of the global polarization vector (left panel) and local density and local director component (right panel) as a function of global polarization $P$. Same simulation as in Fig. 4.18b. 


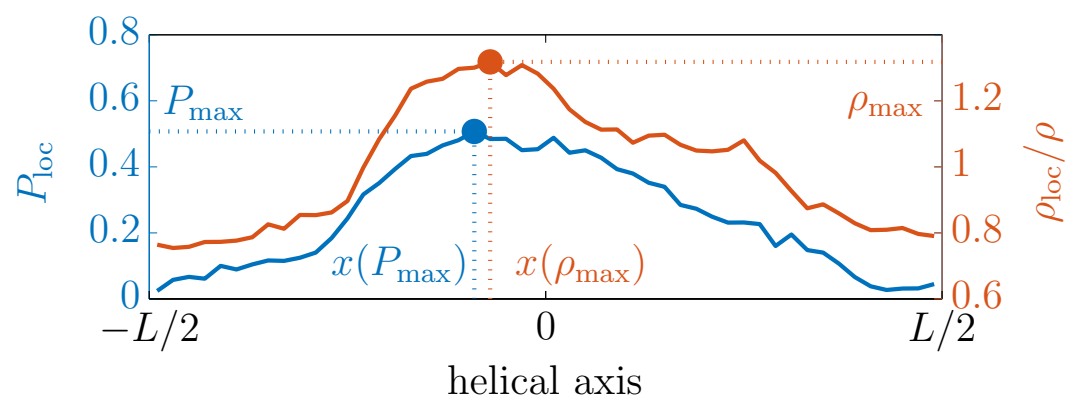

(a) The profiles of local polarization and local density along the helical axis show a clear maximum (for a given time instant) which are denoted $P_{\max }$ and $\rho_{\max }$ with the positions $x\left(P_{\max }\right)$ and $x\left(\rho_{\max }\right)$.
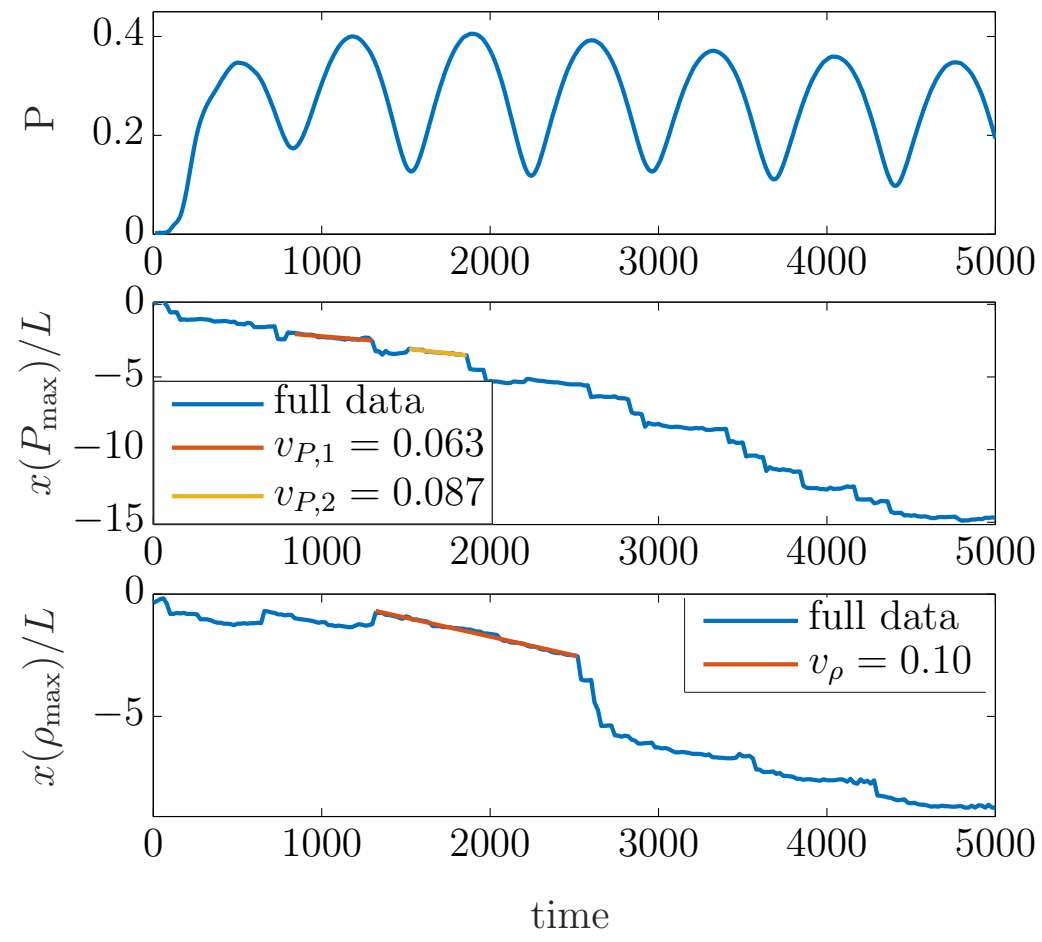

(b) Temporal evolution of the global polarization, as well as the maxima in local polarization and local density (as defined above). The images of the simulation box according to PBCs are taken into account, so that the maximum can be followed on a longer distance than the linear box size. The red and yellow curves are linear fits $x\left(P_{\max }\right)=x_{P}^{0}+v_{P} \cdot t$ (and accordingly for $\rho_{\text {loc }}$ ) in the indicated regions.

Figure 4.20: Measurement of the wavespeed of local polarization and local density (same data as in Fig. 4.18). 
of one oscillation period. In contrast, the profile of the local density changes from a rather flat profile (for low global polarization) to a profile with a distinct minimum and maximum (for high values of the polarization). These profiles hence show that the helix of local directors only shows a very slow rotation (small changes in $d_{\mathrm{loc}, y}(x)$ ) which is much slower than the oscillation of polarization. Hence, the polarization is induced by a density wave.

Finally, we can use our knowledge about the oscillation in polarization and understand how a chiral pattern can untwist (Section 4.4.7). We know now that the oscillating polarization is accompanied by a density wave (along the helical axis). The case of the untwisting chiral (Fig. 4.17) shows an oscillating and increasing polarization order parameter as well as areas of high and low local density in the snapshots $(t=20000$ and $t=35000)$. In a perfect chiral pattern, a difference in local density along the helical axis leads to a higher nematic order parameter as compared to a homogeneous system because the areas of high density contribute more to the nematic order than the ares of low density. Such a density difference hence facilitates the untwisting of a chiral pattern because the stability of the helix relies on a homogeneous density.

\section{Influence of density, Péclet number, and pitch of the helix on the oscillation in polarization}

To understand the full nature of the density wave propagation along the helical axis through the chiral pattern, we analyze the influence of different global quantities onto the period of the oscillation in $P$. This period can be measured either by fitting an oscillating (sinusoidal) function to the data which gives an estimate of the frequency. Secondly, the period can also be found by identifying the maxima of $P(t)$ and measuring the distance between them.

The oscillation period of the polarization $P$ is coupled to the speed with which the density wave travels once across the simulation domain per oscillation period (cf. Fig. 4.18). We find (see Fig. 4.21) that the Péclet number, the global density, and the pitch of the helix influence the period. The first two quantities are studied in a cubic domain while the simulation box to study the influence of the pitch is elongated. The simulations which are used to study the effect of the Péclet number are the same as those that were used to investigate the probability of the formation of a chiral pattern (see Tab. 4.2). Hence, the chiral pattern formed spontaneously. However, to increase the efficiency and to limit the number of performed simulations, in the cases of determining the influence of density and pitch seeded simulations have been used. This means that the simulations do not evolve from an isotropic configuration but in contrast to the previous develop from a perfect chiral pattern. The particles are placed homogeneously in the simulation domain during the initialization step and their orientation is given by $\hat{e}=\left(0 ; \cos \left(n \pi / L_{y} x\right) ; \sin \left(n \pi / L_{y} x\right)\right.$, hence the helical axis 


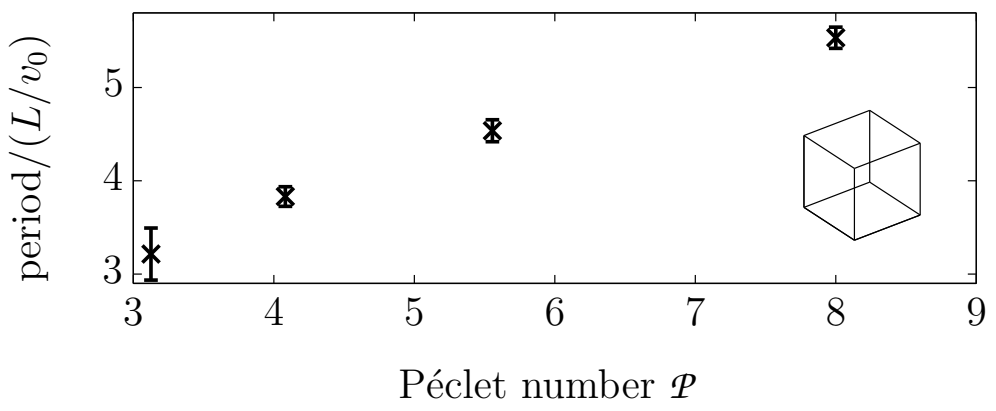

(a) Influence of the Péclet number. $\left(N=66^{3}, \rho=1\right.$, cubic box, same data as in Tab. 4.2, between 9 and 18 independent simulations per data point)

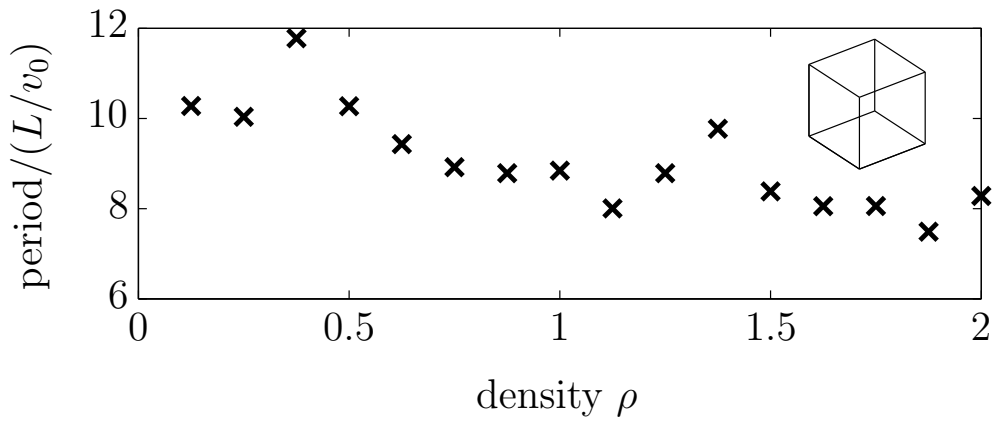

(b) Influence of the global density. $\left(N=66^{3}, \mathcal{P}=22.2\right.$, cubic box $)$

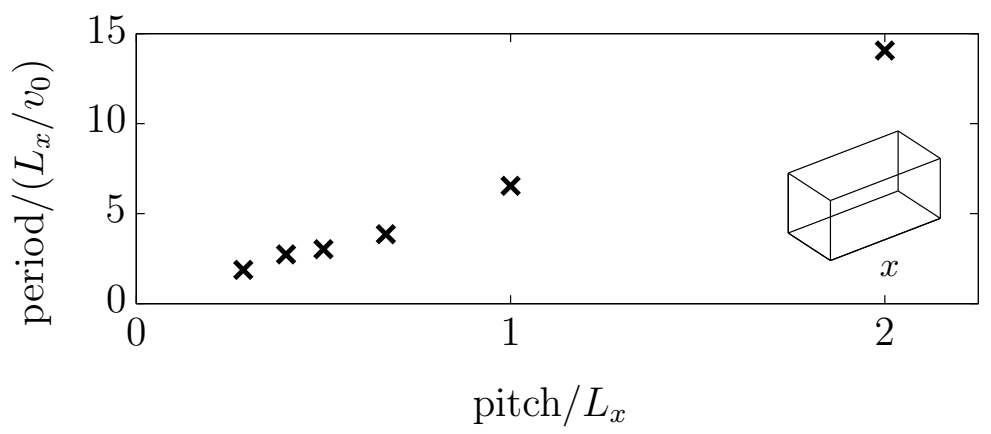

(c) Influence of the pitch of the helix. $\left(N=80 \times 40^{2}, \rho=1\right.$, elongated box $)$

Figure 4.21: Influence of different quantities on the oscillation period of the global polarization of chiral pattern. 
is parallel to $\hat{x}$. The number $n$ sets the pitch as $2 L_{y} / n$. The simulations at constant Péclet number for different densities are performed in a cubic box with a pitch of twice the linear box size. The inspection of the order parameters reveals the time at which the steady state is reached and measurements can be performed. Moreover, the chiral pattern is stable in all cases and does not untwist. The influence of the pitch is investigated in an elongated box of aspect ratio $2: 1: 1\left(L_{x}=2 L_{y}=\right.$ $\left.2 L_{z}\right)$. After a short transient, the polarization shows an oscillation until the pattern untwists and either becomes nematic or chiral with a pitch of $2 L_{x}=4 L_{y}$. Nonetheless, measurements are possible in the time span which correspond to a chiral pattern with a smaller twist.

The measured period of oscillation of $P$ varies between 1.8 and 10 times the time it takes a single particle to travel once across the simulation box along the helical axis $\left(L_{x} / v_{0}\right)$. The period increases with increasing Péclet number (Fig: 4.21a), i.e. the density wave gets slower with increasing rotational noise. The period, hence, follows the same trend as a function of $\mathcal{P}$ as the global nematic order parameter. In the limit of infinite Péclet number and perfect order (given that the non-dimensional density is large enough for global order to occur) the system cannot exhibit a density wave propagating perpendicular to the global director ${ }^{7}$. For a fixed Péclet number, the period of the oscillation in polarization decreases with increasing global density (see Fig. 4.21b). This trend is opposite to the trend of the global nematic order parameter which increases with increasing number density. However, the decrease of the period is rather small (between ten and eight times $L / v_{0}$ ) over non-dimensional number densities in the range $0.125 \leq \rho \epsilon^{3} \leq 2$. The density wave can travel faster (leading to a smaller period) for higher number densities since more interactions happen when the system is denser. Finally, to understand the interplay between the density wave and the chiral pattern, we perform simulations with different pitches (see Fig. 4.21c). The period clearly increases from roughly $1.9 L_{x} / v_{0}$ to $14 L_{x} / v_{0}$ for the maximum possible pitch of $2 L_{x}=4 L_{y}$. This indicates that the density wave gets considerably faster the more the helix of local directors is twisted. This could be related to the curvature induced instability which was found by Baskaran and Marchetti (2008b, 2012) deep in the nematic phase of the nonequilibrium phase diagram.

To summarize, we observe a density wave associated with the chiral pattern which propagates along the helical axis and is coupled to an increase in the local polarization. Its speed depends on the global density, the Péclet number, and the pitch of the helix.

\subsection{Influence of walls}

All simulations up to this point are performed with periodic boundary conditions. However, in Section 3.2.4 we describe two different models to implement a sandwich

\footnotetext{
${ }^{7}$ At the same time also a chiral pattern is not able to form in the limit $\mathcal{P} \rightarrow \infty$ (see Section 4.4.6).
} 


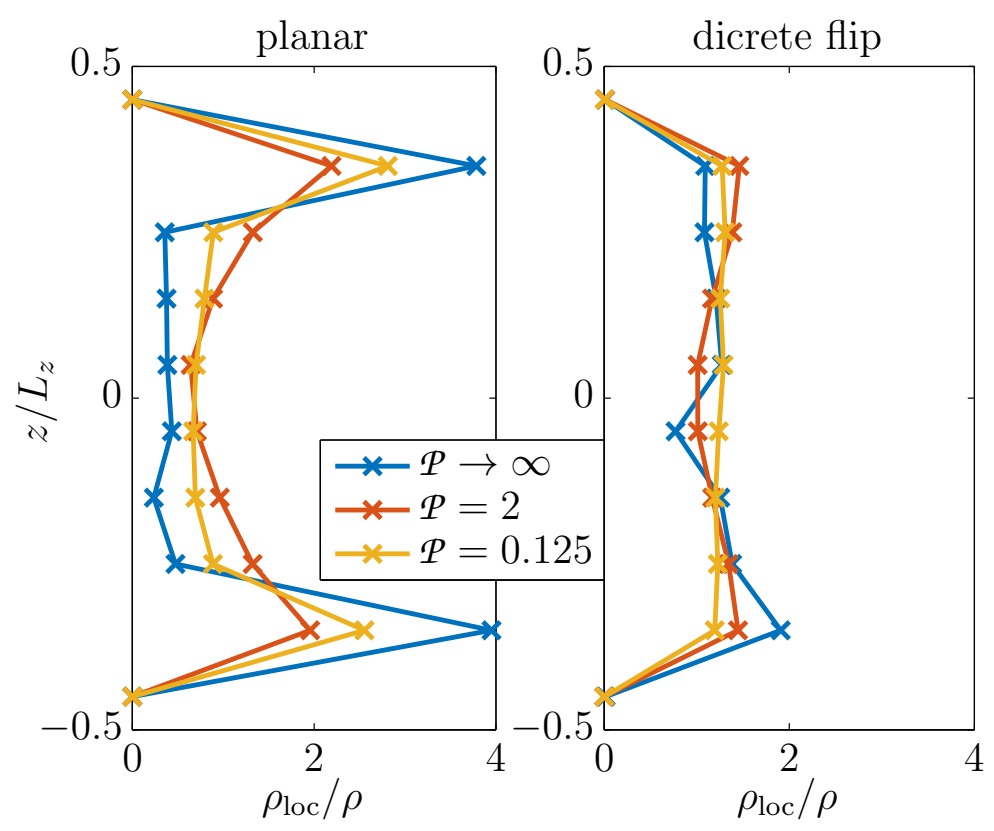

Figure 4.22: Comparison of the two models for the sandwich geometry. The local number density is calculated in 10 boxes along the $z$-axis $\left(N=1000, \sigma / L_{z}=0.1\right)$.

geometry. In the following we want to study the influence of such boundaries.

We perform simulations of a small system $(N=1000, \rho=1)$ and investigate the vertical density profile which measures the local density in layers parallel to the walls (Fig. 4.22). Both models show a vanishing local densities close to the walls due to the repulsion (Eq. 3.50).

The model with planar anchoring (Eq. 3.52) shows large accumulations of particles close to the walls (Fig. 4.22, left panel). This effect is strongest for the deterministic model $(\mathcal{P} \rightarrow \infty)$, followed by the isotropic phase $(\mathscr{P}=0.125)$, and it is weakest in the nematic phase $(\mathbb{P}=2)$. The wall-accumulation is directly induced by the anchoring mechanism: at the top wall all particles align along $\hat{x}$ so that it is hardly possible for a particle to leave the vicinity of the wall again. At the bottom wall, the inter-particle alignment can lead to local alignment of all particles close to the wall which again keeps all these particles together. The wall-accumulation is strongest for the deterministic system $(\mathcal{P} \rightarrow \infty)$ where particles are strongly trapped at the walls because no rotational noise is applied. The weakest accumulation is found for the nematic phase because the walls will induce a global alignment perpendicular to the $z$-axis preventing particles from approaching the walls. On the contrary in the isotropic phase, the orientations of the particles are equally distributed into all directions which means that at all times particles are approaching the walls. At the same time the particles being already close to the walls are subject to stochastic 


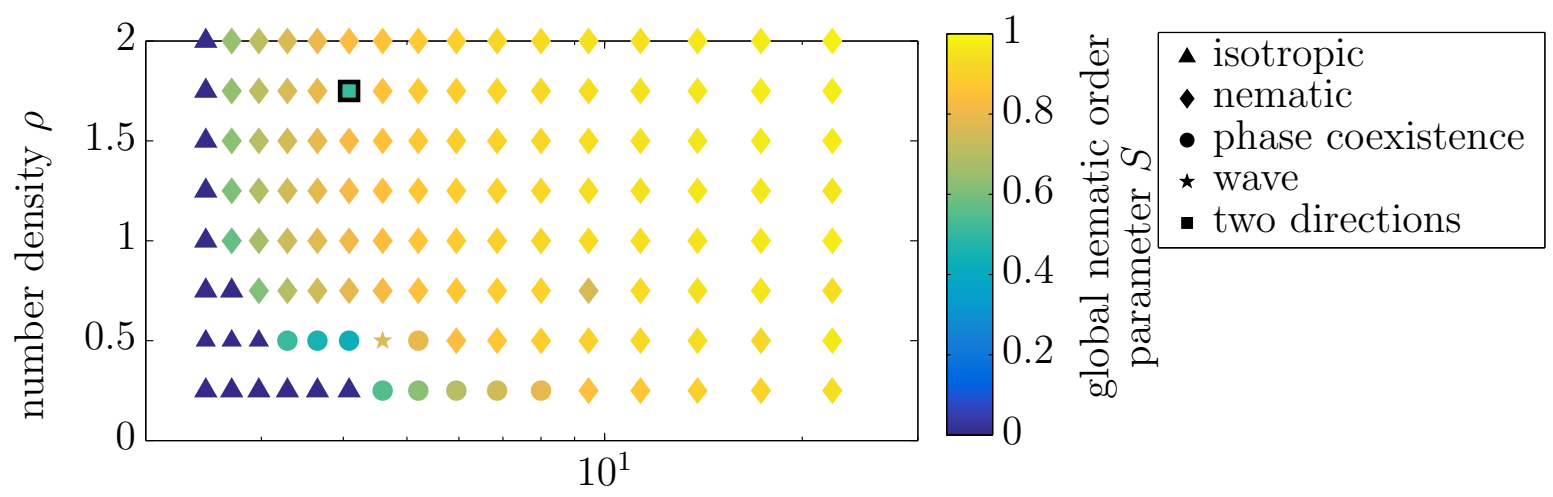

Péclet number $\mathcal{P}$

Figure 4.23: Nonequilibrium phase diagram (analogous to Fig. 4.1) of SPPs in a sandwich geometry using the discrete flip model $\left(\gamma=0.1,24 \epsilon_{1} / \gamma_{F}=0.1\right)$.

rotational noise which can possibly take them away from the wall. However, these two effects do not cancel out because the particle-wall alignment mechanism traps the particles at the walls.

The second model for SPPs in a sandwich geometry is termed "dicrete flip" (Eq. 3.54) and shows a homogeneous distribution of the local density (Fig. 4.22, right panel). The resulting density profile does not change for different Péclet numbers. We hence use this model in the following to investigate the influence of walls onto aligning SPPs.

The phase diagram (Fig. 4.23) is very similar to the one of the particles in a simulation box with periodic boundary conditions (Fig. 4.1). Mainly two domains can be identified: An isotropic phase forms for low Péclet number and a nematic phase evolves where the Péclet number is high. The transition line again has a negative slope in the $(\rho, \mathcal{P})$-plane so that the critical Péclet number decreases with increasing global density. Close to the transition line (and especially at low densities) the phase coexistence and a traveling wave are found.

The main difference of the phase diagram of the system with walls compared to the periodic boundary system is the absence of the chiral simulations scattered in the nematic phase. However, out of all performed simulations, we find one in the nematic phase ( $P=4.1, \rho=1.75)$ which shows a considerably smaller global nematic order parameter than all surrounding simulations. Visual inspection of the corresponding snapshot (Fig. 4.24b) reveals a very interesting pattern: The simulation box is subdivided into several distinct parts. More than half of the box is populated by a rather dense, locally nematically ordered "stream" of particles with the local director being aligned perpendicular to the walls. The rest of the box is rather empty with only a few particles which do not show nematic alignment on a larger scale except for a second dense "stream" close to the bottom wall of the simulation box oriented along the simulation box. The two local nematic directors of the two "streams" hence form 


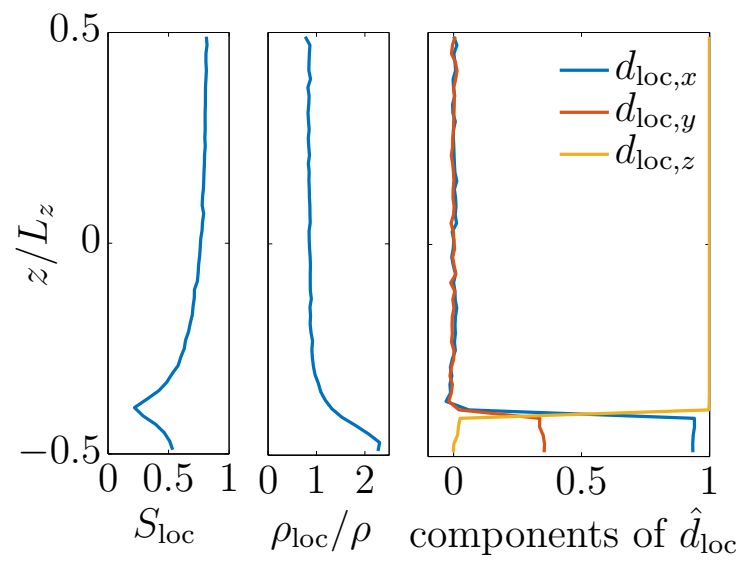

(a) Vertical profiles (measured in 50 slices) of local nematic order parameter, local density, and components of local nematic director.

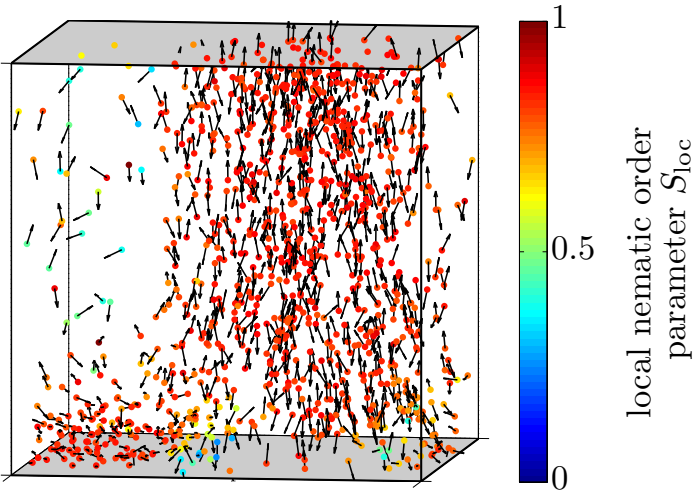

(b) Snapshot with local nematic order parameter calculated in $20^{3}$ boxes (like in Fig. 4.2)

Figure 4.24: Two-directional pattern in the system with sandwich geometry $(\mathcal{P}=4.1$, $\rho=1.75)$.

an angle of $\pi / 2$. For further analysis the vertical profiles of local density and local nematic order are considered: The simulation domain is subdivided into 50 equally spaced, horizontal slices to calculate the local density, local nematic order, as well as the local director within each slice (see Fig. 4.24a). The vertical profiles of the components of the local nematic director substantiate what we described from the visual inspection of the snapshot: The local director almost everywhere in the box is aligned along the $z$-axis, i.e. the particles move perpendicular to the walls. The lower part of the simulation domain, however, shows a local alignment in the $x-y$ plane (vanishing $z$ component of $\hat{d}_{\text {loc }}$ ) but not along one of the axes. The vertical profile of the local density is homogeneous in the upper part of the box and increases towards the lower wall. It is a gradual increase and not a sharp transition like for the components of the local director. The shape of the vertical profile of the local density reflects the fact that close to the bottom wall a second "stream" of particles is formed next to the vertical one so that the number of particles in a horizontal slice increases. The local nematic order parameter shows a non-monotonic vertical profile with a high value $\left(S_{\text {loc }} \approx 0.8\right)$ in the upper part of the simulation domain where the particles are mostly aligned vertically. A minimum of $S_{\text {loc }}$ is reached where the local director transitions from vertical to horizontal alignment and hence the system appears disordered. Close to the lower wall the nematic order parameter increases again with a maximum value of $S_{\text {loc }} \approx 0.5$ in the vicinity of the wall where the horizontal "stream" dominates. 


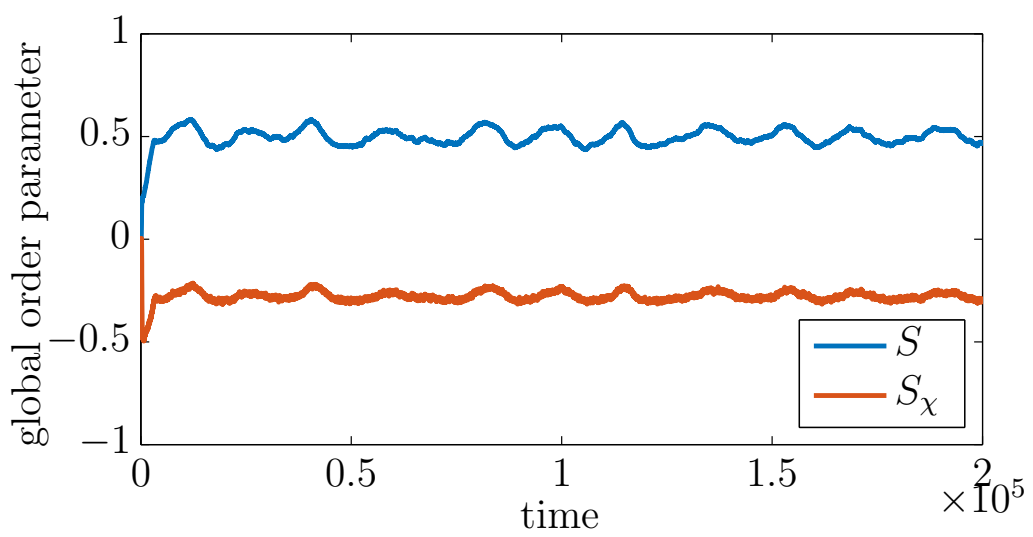

Figure 4.25: Temporal evolution of the global order parameters in the simulation which shows two main directions of local nematic directors (sandwich geometry, see Fig. 4.24).

The temporal evolution of the nematic and chiral order parameters shows a surprising stability of this configuration (Fig. 4.25): it lasts for at least $2 \times 10^{6}$ time steps $(\Delta t=0.1)$ which is comparable to the stability of the chiral pattern in the system without walls (Fig. 4.11a). Both order parameters fluctuate in time around intermediate values of $S \approx 0.5$ and $S_{\chi} \approx-0.3$. These values reflect the partial nematic order in the system, as well as the occurrence of a chiral pattern even though the particles do not form a helix like in the case with PBCs. We find that the walls suppress the formation of chiral patterns in terms of helices but on rare occasions a chiral pattern can emerge in the nematic area of the phase diagram which is composed of differently oriented, nematically ordered domains.

\subsection{Discussion}

In this chapter, we have studied a simple model which shows a surprising variety of steady-state patterns despite the fact that it consists of only few ingredients: selfpropulsion, local nematic interaction, and rotational diffusion. The system shows a transition from a homogeneous isotropic state to a homogeneous nematic state with increasing Péclet number and number density. However, interesting patterns occur at (or close to) the transition line: The system shows phase coexistence in different geometric arrangements. This resembles the work of Ginelli et al. (2010) who investigated a similar model to ours but in two dimensions. The underlying equations of their model are, however, discrete in contrast to our time-continuous set of equations. Still they see a similar phase coexistence between a dense, nematically ordered band (with the nematic director being oriented along the band) and a dilute, 
isotropic gas. ${ }^{8}$ However, at the transition from nematic to isotropic in the twodimensional system a fluctuating dense band is observed which we do not find in the three-dimensional system. Apart from that the similarity is remarkable and suggests that the two models behave similarly even though they differ in dimensionality and nature of underlying equations of motion (time-discrete vs. time-continuous).

We know from the hydrodynamic theory of nematic SPPs (although again in two dimensions, Baskaran and Marchetti, 2012) that a so-called banding instability is found close to the transition. It can be described as a stationary density wave with the wavevector (and hence the density variations) perpendicular to the global nematic director. The simulations with phase coexistence show the same geometry and suggest that they could be the result of such an instability.

We are able to pin down the transition line and compare the measurements to the result of a mean-field approach. We find good qualitative agreement for low densities. For small nematic relaxation constants even quantitative agreement can be found. At high densities the mean-field approach is not applicable and the critical Péclet number is independent of the number density. The general shape of the transition line is similar to the transition line which was derived by Peshkov et al. (2014) who use an analytical (Boltzmann-Ginzburg-Landau) approach in two dimensions.

Close to the isotropic-nematic transition for low values of the number density we find polarly ordered, dense, propagating waves. Their maximum wavelength is predefined by the nematic director but still we often find several wavetrains propagating into the same direction. Typically these wavetrains self-assemble such that they appear equally spaced along their direction of motion. The resulting wavelength is then a simple fraction (half, third, ...) of the maximum wavelength. The wavelength is hence restricted by the geometric orientation of the wave in the simulation box with periodic boundary conditions but the system still shows its intrinsic dynamics by this self-organization process. The global nematic order of the system is usually preserved because for each emerging wave also a counter-propagating wave forms. However, we find that for a large nematic relaxation constant $\gamma \geq v_{0} / \epsilon$ the region in the nonequilibrium phase diagram corresponding to the waves is subdivided into two distinct regions in terms of the polar order parameter: At low number density global polar order emerges which means that the waves only propagate into one direction and there are no counter-propagating waves. While the waves themselves only break the nematic symmetry locally, the absence of counter-propagating waves indicates even a symmetry breaking of global nematic order.

Our simulations show that polar, propagating density waves can emerge in a threedimensional system of nematically aligning SPPs and not only in two-dimensional systems of either polarly aligning or extended particles (without explicit interaction

\footnotetext{
${ }^{8}$ Similar patterns are also observed in a twodimensional system of self-propelled rods with velocity reversal (Großmann et al., 2016).
} 
rule). They resemble very closely the density waves emerging in simulations of the original Vicsek model and which are even predicted by kinetic theory (Ihle, 2013). These waves also occur close to the order-disorder phase transition and show a similar density profile. Hydrodynamic theory as well results in an instability due to the buildup of local polar order which leads to density variations along the nematic director (Baskaran and Marchetti, 2012). The local polar order is induced by an asymmetry of the interactions between nearly aligning and nearly anti-aligning particles. This asymmetry is generated by the momentum conservation of hydrodynamically interacting self-propelled particles and favors the parallel alignment of particles as opposed to anti-parallel alignment. The interactions in our system do not conserve momentum but still can lead to a build-up of polar order: If two nearly anti-parallel particles interact, they align and then clearly move away from each other. On the other hand, two nearly parallel particles align and stick together if the alignment mechanism is stronger than the rotational noise. The tendency of aligning particles to stick together depends on the ratio of two time scales: The time scale of nematic alignment and the time it takes a particle to travel a distance further than the nematic interaction range. This ratio is given by $\gamma /\left(v_{0} / \epsilon\right)$. We learn from our simulations that if this ratio is larger than unity (and hence, the time scale of the alignment mechanism is shorter than the time it takes a particle to travel across the nematic interaction range), the region of the phase diagram where waves occur is larger than for small $\gamma /\left(v_{0} / \epsilon\right)$, and the above described mechanism can even lead to global polar order. Moreover, the field of local density is coupled to the local polarization through this mechanism since the alignment mechanism serves effectively as an attractive interaction between particles. This coupling is also the reason why the system shows global polar order for low densities (and large $\gamma /\left(v_{0} / \epsilon\right)$ ). All of these reasons suggest that our waves are indeed an instability in the nematic phase due to the build-up of local polar order. The formation of the waves substantiates this claim because the waves are forming gradually from a homogeneous, nematic state.

The second very interesting finding in our system of self-propelled, nematically aligning particles is the formation of a chiral pattern in the form of a helix formed by the local nematic director. This is, to the best of our knowledge, the first observation of such an emerging helical structure in a system of active particles. It is fundamentally different from other chiral structures such as swarm rotation because a single particle in our chiral pattern still moves roughly in a straight line whereas individual particles in a rotating swarm follow curved paths. Our chiral pattern shows signs of a spontaneous symmetry breaking and emerges with a certain probability in the nematic region of the phase diagram independent of the (homogeneous and isotropic) initial conditions. We show that its precursor are roughly parallel, nematically ordered planes with non-parallel local nematic directors, and we find that the rotational diffusion of the particles is crucial for the formation of a chiral state. The helical pattern is long-lived and metastable but can possibly untwist. This relaxation 
of a twist state to the nematic ground state of the system is coupled to a very regular oscillation of the global polarization. This oscillation originates from a density wave traveling along the helical axis which eventually destroys the chiral pattern: If the helical pattern is subject to a density variation along the helical axis, the overall structure is closer to a nematic state than a homogeneous helix because the particle orientations are not equally distributed. In the limit of a very strong density fluctuation this can destroy the chiral pattern. Again, as for the wave pattern, the build-up of polarization is an important feature of the system.

In the two-dimensional system a curvature-induced instability has been derived from hydrodynamic theory (Baskaran and Marchetti, 2008b, 2012) which take into account bend and splay deformations of the director field. Clearly, a twist deformation like our chiral pattern can only occur in three dimensions and so an extension of the hydrodynamic model to three dimensions would be necessary to investigate whether a twist-related instability exists.

We compare our model to a one-dimensional model of rotors on a chain which resembles the classical $X Y$-model but with nematic symmetry. Under rapid nonequilibrium quenches this system also exhibits chiral states with different numbers of twists. Depending on the size of the system the chiral state can be more probable than the nematic state. Actually the number of twists of the most probable state grows with the system size and eventually destroys long-range order in the infinite system.

Introducing reflective boundaries to the system in one direction (sandwich geometry) does not change the phase diagram substantially but only suppresses the formation of helices. However, the formation of a chiral pattern is observed: Two distinct, nematically aligned streams of particles form an angle of $\pi / 2$.

Because the periodic boundary conditions are important for the formation, stability, and properties of the helical state an open question is the emergence and relevance of this state in bulk. Besides this criticism the emergence of chiral patterns still gives us an interesting insight into how chiral patterns form from a completely achiral system. This is our small contribution to the question of how chirality can emerge and its repercussions on the key ingredients of living matter. 



\section{Structure formation by self-propelled extended particles}

The subject of the previous chapter (Chapter 4) are point-like, self-propelled, nematically aligning particles in three dimensions. However, in reality such particles will always possess a finite extension. It is hence more realistic to also model them as such. The following short chapter shows results of such self-propelled particles, their nonequilibrium phase diagram with typically occurring phases. We especially emphasize the differences to point-like particles.

The extended particles are modeled using the WCA potential to include a hard core. We solve the equations of motion (Eq. 3.5) using the Euler-Maruyama scheme described in Eq. (3.35) with PBCs. They differ from the equations of motion for point-like particles (Eq. 3.2) by two additional terms: The steric interaction between particles leads to repulsion between particles which are closer than a cutoff distance $r_{0}=\sqrt[6]{2} \sigma$ (where $\sigma$ is the particle diameter). Moreover, the WCA force also enters the equation of motion of the orientation to mimic the additional torque which extended elongated particles exert on each other. This is an approximation because our particles are not truly elongated but spherical with an intrinsic orientation. Our typical model parameters can be found in Tab. 5.1. We use fewer particles than in the simulations with point-like particles to achieve reasonable computation times because reaching the steady state requires a larger number of time steps since $\Delta t$ is smaller for extended particles than for point-like particles. Such a smaller time steps has to be chosen in order to properly resolve the interparticle collisions according to the WCA potential. The particle diameter is $\sigma=0.445 \epsilon$ such that $r_{0}=\sqrt[6]{2} \sigma=\epsilon / 2$. 


\begin{tabular}{ll}
\hline parameter & value \\
\hline number of particles & $N=50^{3}=125000$ \\
side lengths of simulations box & $L=L_{x}=L_{y}=L_{z}=\sqrt[3]{\frac{N}{\rho}}$ \\
nematic interaction range & $\epsilon=1$ \\
particle diameter & $\sigma=0.445$ \\
relaxation constant of WCA potential & $\gamma_{F}=1$ \\
strength of WCA potential & $\epsilon_{F}=0.01$ \\
self-propulsion speed & $v_{0}=0.5$ \\
nematic relaxation constant & $\gamma=0.1$ \\
rotational diffusion & $D_{r}=\frac{\gamma}{p}$ \\
time step & $\Delta t=0.01$ \\
\hline
\end{tabular}

Table 5.1: Input parameters for extended self-propelled particles.

\subsection{Nonequilibrium phase diagram and new patterns}

Analogously to point-like particles, we investigate the nonequilibrium phase diagram of the system of extended particles and measure the global nematic order parameter as a function of Péclet number $\mathcal{P}$ and number density $\rho$ (Fig. 5.1a). For extended particles it is also useful to define the filling fraction

$$
\begin{aligned}
\Phi & \equiv \frac{\text { volume occupied by particles }}{\text { total volume of system }} \\
& =\frac{N(\sigma / 2)^{3} 4 \pi / 3}{L^{3}} \\
& =\frac{\pi}{6} \sigma^{3} \frac{N}{L^{3}}=\frac{\pi}{6} \sigma^{3} \rho
\end{aligned}
$$

to estimate how closely packed a given configuration is. The phase diagram of extended particles resembles that of point-like particles: At low Péclet numbers an isotropic state occurs, while the system exhibits global nematic order at high Péclet numbers. A narrow transition domain is found where isotropic and nematically ordered domains can coexist in one and the same system. However, the pattern differs from the phase coexistence state of point-like particles as described in Section 4.1. We will discuss this new pattern in detail in the following section. The extended particles also exhibit chiral patterns of the same kind as the chiral pattern of point-like particles (see Section 4.4) which occur randomly in the nematic part of the phase diagram. No propagating waves (see Section 4.3) were observed in the given ranges of Péclet number and density. The critical Péclet number (defined as the smallest Péclet number where nematic order is observed) decreases with increasing number 


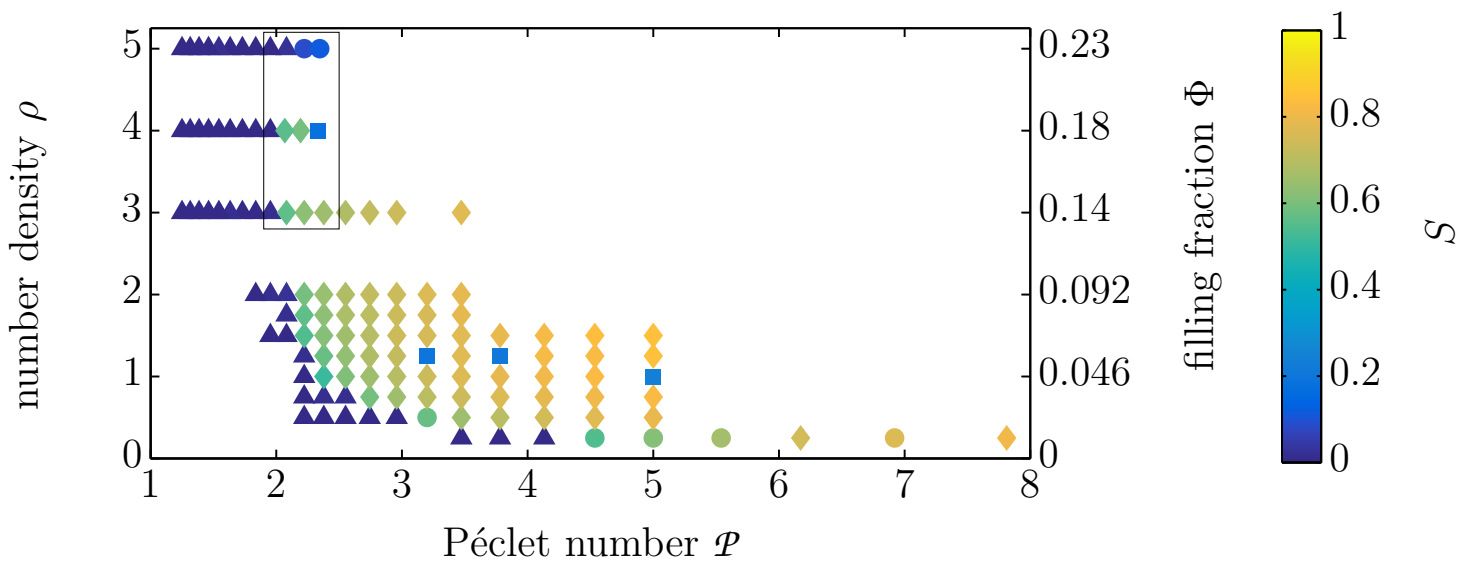

(a) Nematic order parameter (in color) as a function of Péclet number and number density $\rho$ or filling fraction $\Phi$ (see Eq. 5.1). The black rectangle indicates the region which is shown in detail below.

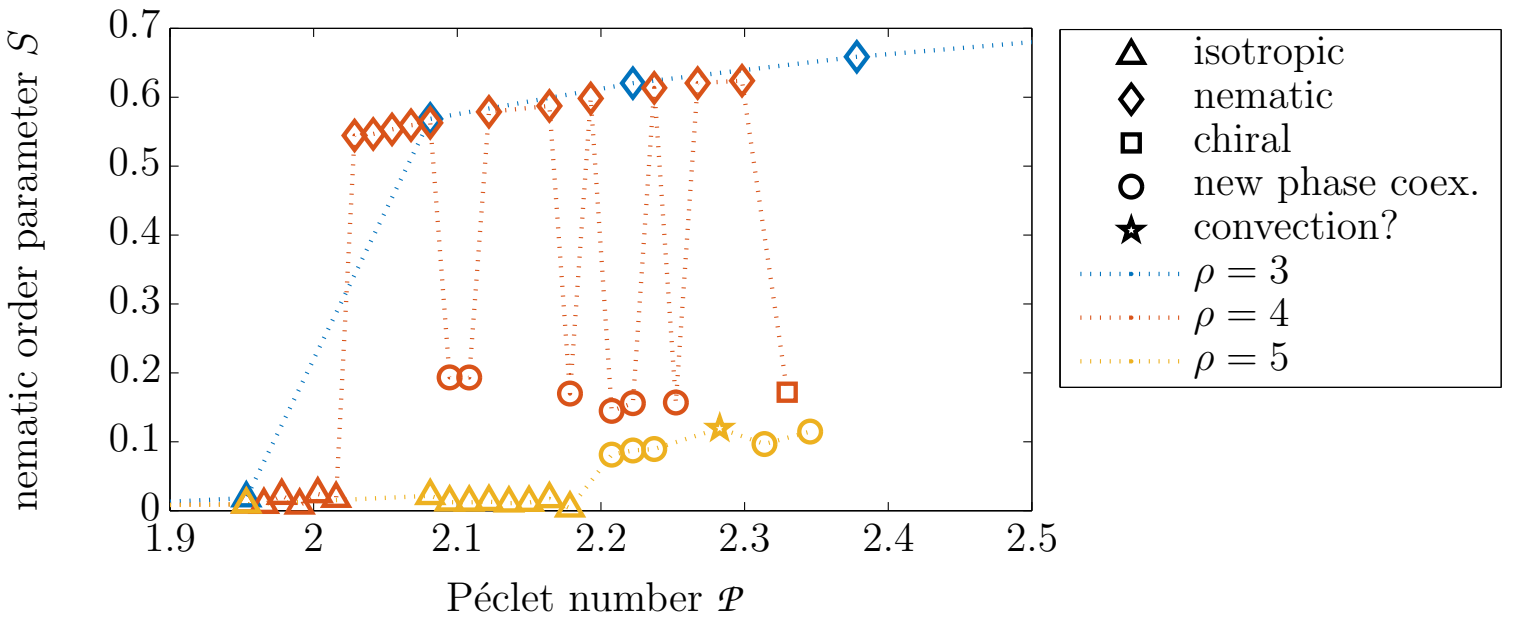

(b) Nematic order parameter as a function of Péclet number for the three largest densities investigated as indicated by the black rectangle in the plot above.

Figure 5.1: Nonequilibrium phase diagram of extended, self-propelled, nematically aligning particles. Different steady-state patterns are observed and indicated by different symbols (see legend). 


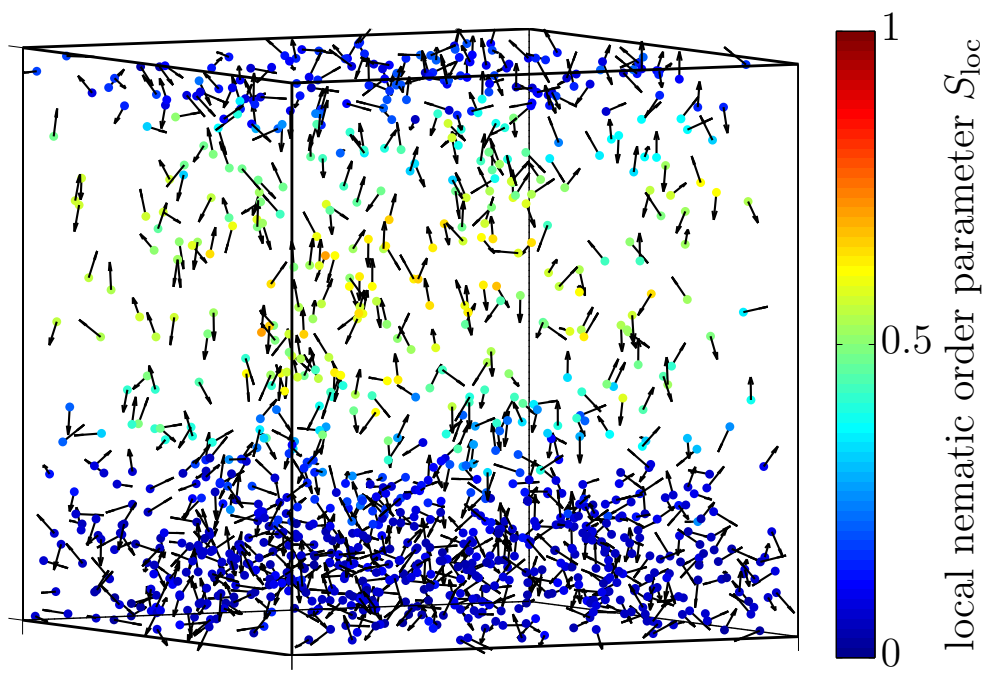

Figure 5.2: Steady-state snapshot of the new phase coexistence pattern occurring in the system of extended particles $(\mathcal{P}=2.22, \rho=4)$ which does not occur for point-like particles. Periodic boundary conditions are applied in all three dimensions. No solid walls are present. For the sake of clarity we show only $0.8 \%$ of the particles with the local nematic order parameter in color and the local director as little arrow. The side length of the simulation box is $L \approx 71 \sigma$.

density similarly as for point-like particles (Section 4.2). However, for high number densities $\rho \geq 3$ and filling fractions $\Phi \geq 0.14$ the behavior of the system appears to change. We investigate in detail the isotropic-nematic transition for these densities in Fig. 5.1b. At a number density of $\rho=3$, the system shows a clear transition from the isotropic state to the nematic state with increasing Péclet number just like for smaller densities. If the density is increased to $\rho=4$ such a transition occurs as well. However, in the nematic phase the system for some Péclet numbers exhibits the new phase coexistence state instead of a fully nematic system. The nematic order parameter as a function of Péclet number shows abrupt jumps because the nematic order parameter of the phase coexistence state is much lower $(S \approx 0.2)$ than that of the nematic state $(S \approx 0.6)$. At the highest investigated number density $(\rho=5)$, the homogeneous, nematic state is suppressed and we observe a transition from the isotropic state to the new phase coexistence state. One of these simulations shows an interesting pattern which resembles a convection role. We will investigate it in Section 5.1.2. 


\subsubsection{New phase coexistence}

Figure 5.2 shows a typical snapshot of the new phase coexistence pattern. ${ }^{1}$ Vertical profiles of the nematic order parameter $S_{\text {loc }}$ and of the local density $\rho_{\text {loc }}$ are calculated from the same data (see Figs. 5.3a and 5.3b). We see indeed phase coexistence between a nematically ordered domain and an isotropic domain. However, the nematic domain is dilute while the particles in the isotropic domain are rather densely packed. This negative correlation between nematic order and density is opposite to what we found in the system of point-like particles where the areas of highest local density would correlate with the areas of strongest local nematic order. The nematic domain percolates just like for point-like particles but the local nematic director is oriented perpendicular to the percolation direction. ${ }^{2}$ Trajectories of individual particles which start in this nematic domain (Fig. 5.3d) reveal that the particles mostly travel straight along the local nematic director (vertical) and are reflected by the isotropic domain. This suggests that the isotropic domain acts like a wall onto the particles. The trajectories of particles which start in the isotropic, dense domain (Fig. 5.3c) display two different kinds of behavior: either the particles are trapped in the dense part of the simulation domain and do not show a persistent direction of motion or they are able to escape from that region and move mostly vertically along the nematic director through the simulation domain.

\subsubsection{Convection?}

We performed simulations up to a density of $\rho=5$. For such a high-density system we find a transition from the isotropic state to the new phase coexistence state with increasing Péclet number (see Fig. 5.1b). The visual inspection of the steady-state configurations reveals that one of the simulations does not show the new phase coexistence pattern as described before with the nematic director perpendicular to the direction of percolation. Instead, the local nematic directors form circular streamlines (see Fig. 5.4) such that a cylindrical configuration emerges. The particles at the center of the cylinder are aligned along the cylindrical axis. A cylinder with circular cross-section cannot fill the whole cubic simulation domain but coexists with a dense, isotropic domain.

\footnotetext{
${ }^{1}$ See also the attached movie hardcores_phase_coexistence.avi.

${ }^{2}$ The orientation of the nematic domain has again to by compatible with PBCs like in the phase coexistence state of point-like particles. Typically it is parallel to one of the axes of the simulation domain but other orientations are observed as well.
} 

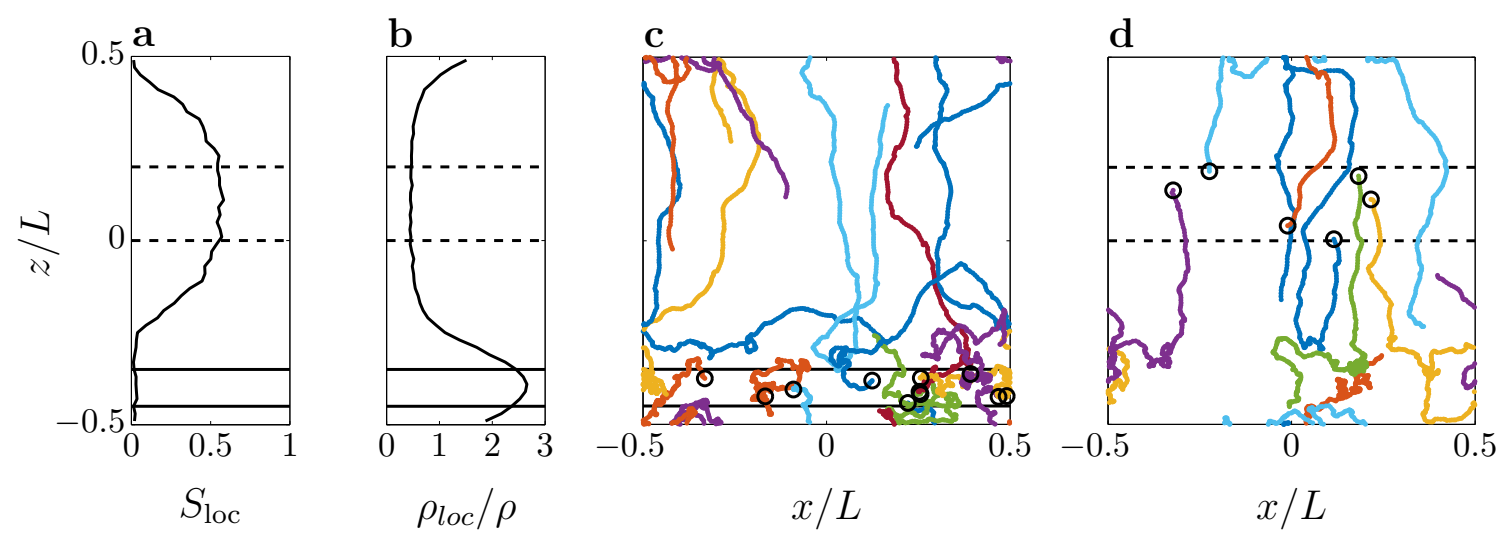

Figure 5.3: Analysis of the new phase coexistence state (see snapshot in Fig. 5.2). The vertical profiles of local nematic order parameter $S_{\text {loc }}\left(\right.$ a) and local density $\rho_{\text {loc }}$ are calculated from 50 horizontal slices of the simulation domain. The dashed (solid) lines mark the nematically ordered and dilute (isotropic and dense) domains of the system. Panels $\mathrm{c}$ and $\mathrm{d}$ show side views of the simulation domain with trajectories of a few individual particles which start in the dense, isotropic domain (c) or in the dilute, nematic domain $(d)$.

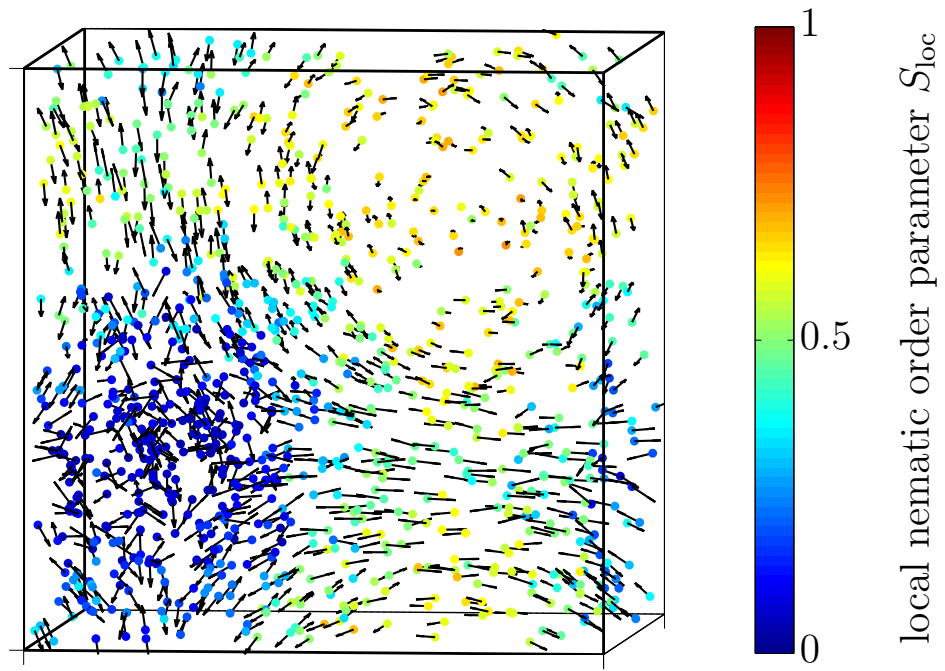

Figure 5.4: Steady-state configuration of a system which exhibits a convection pattern $(\rho=5, \mathcal{P}=2.28)$. See Fig. 5.2 for plotting details. Here, the side length of the simulation domain is $L \approx 66 \sigma$. 


\subsection{Discussion}

Our model of self-propelled, aligning particles can be extended by introducing steric interactions among particles. The phase diagram in terms of the global nematic order parameter as a function of Péclet number and density shows the same qualitative behavior as for point particles. Deviations from this can be found at large densities. At low densities the steric interactions are not as important as at large densities because particles impinge against each other less frequently. The phase diagram of a low-density suspension of extended particles should thus be governed by the phase diagram of point-like particles.

One difference at all densities between the two models is the occurrence of different phase coexistence patterns: For point-like particles local nematic order and local density are positively correlated while the correlation is negative for extended particles. Also the orientation of the local director with respect to the percolation direction differs in both cases: For point-like particles $\hat{d}_{\text {loc }}$ is perpendicular to the layer normal of the nematic layer while the two vectors are parallel for extended particles. The reason can be found in the equation of motion of extended particles: the steric interaction does not only lead to a repulsion of neighboring particles but also affects the mutual orientations. A given particle in a region of high local density is subject to orientational changes due to all of its neighbors. This can be viewed as an additional rotational noise if these neighbors are placed homogeneously around the particle because all contributions sum up. In contrast the Lebwohl-Lasher potential includes a term $1 / n_{i}$ where $n_{i}$ is the number of neighboring particles of particle $i$. The individual two-particle alignment interactions are, therefore, averaged rather than summed up. As a result the influence of the alignment compared to the orientational changes due to the WCA force decreases with increasing local density, and dense domains of the system will be isotropic while dilute parts can achieve nematic order. This interplay between the different terms in the equations of motion of $\hat{e}$ might also be the reason that we do not observe any dense, propagating waves (like they are investigated in Section 4.3 for point-like particles). The given system is not able to form and sustain dense, nematically ordered domains and also polarly ordered domains like in the waves are not possible. Our model of extended particles aims to model the behavior of rod-like particles with steric interactions and alignment. This could be improved by introducing actual elongated particles like it is done in the field of liquid crystals (Ilnytskyi and Wilson, 2002). Such an approach would also allow to study the effect of the particles' aspect ratio in our model. It has been shown experimentally using rod-like bacteria that the swarming behavior depends crucially on the aspect ratio (Ilkanaiv et al., 2017).

The convection-like pattern was observed only once in the phase diagram so it is impossible to make a statement about its probability. However, the spontaneous formation of such a cylindrical pattern is interesting in itself. It resembles convection 
rolls like they are found in Rayleigh-Bénard convection. Since our particles are selfpropelled a connection to the field of bioconvection (Platt, 1961; Hill and Pedley, 2005) is appealing. However, true convection patterns need an external forcing like a temperature gradient or the field of gravity which drives motion due to a density mismatch between the local and global densities. Our system is not subject to any external force and still self-organized into a similar pattern.

A completely different set of systems where similar patterns occur are self-propelled particles in confinement: Dense bacterial suspensions in a circular confinement selforganize into circular patterns (Lushi et al., 2014). However, our pattern does not occur in a confined geometry but in a cubic simulation domain with periodic boundary conditions. Further investigation is necessary to understand the formation, stability, and probability of such a pattern. 


\section{Self-propelled particles in a turbulent field}

The subject of this chapter are self-propelled particles which are immersed in a surrounding turbulent fluid. The fluid acts as a field interacting with their positions and orientations: the particles are advected and the turbulent vorticity exerts a torque onto the orientations (see Eq. 3.7). In the following, we will analyze three different models: Point particles in a Kraichnan flow field, extended particles in a Kraichnan flow field, and point particles in a DNS flow field. The input parameters (if not stated otherwise explicitly) are given in Tab. 6.1. The nematic interaction range is chosen such that $\rho \epsilon^{3}=N \epsilon^{3} / L^{3}=1$. The packing fraction of the simulations with extended particles is $\phi=4 / 3 \pi(\sigma / 2)^{3} N / L^{3} \approx 6.54 \%$. The stochastic noise $\eta$ as well as the nematic relaxation constant $\gamma$ will be changed to achieve different values of Péclet and vortical Stokes number.

This chapter is organized as follows: First the phase diagrams of all three models in terms of the nematic order parameter are shown and the nematic-isotropic transition is investigated. Then the occurrence of turbulence-induced clustering is studied using different analysis methods. Additionally, the temporal evolution of the clustering is analyzed as well as the influences of different model parameters onto the formation of small-scale patches. Finally, the results are discussed with particular attention to the mechanism which leads to the clustering. 


$\left.\begin{array}{ll}\hline \text { parameter } & \text { value } \\ \hline \text { number of particles } & N=30^{3}=27000 \\ \text { side lengths of simulation box } & L=L_{x}=L_{y}=L_{z}=2 \pi \\ \text { nematic interaction range } & \epsilon=0.21 \\ \text { self-propulsion speed } & v_{0}=8 \\ \text { time step } & \Delta t=0.0069 \text { (point particles) } \\ & \Delta t=10^{-4} \text { (extended particles) } \\ \text { strength of turbulent field } & E_{0}=0.35 \\ \text { number of Fourier modes } & N_{F}=64\end{array}\right\}$ general

Table 6.1: Input parameters for the simulations of particles in a turbulent field (if not stated otherwise).

\subsection{Nematic Order}

The interaction between neighboring particles is nematic, so we first need to understand the nonequilibrium phase diagram of the system.

\subsubsection{Phase diagrams}

The global nematic order parameter as a function of Péclet number and vortical Stokes number is given in Fig. 6.1. All three models (point or extended particles in Kraichnan flow field and point particles in DNS flow field) are isotropic $(S \rightarrow 0)$ for small $\mathcal{P}$ or small $\mathcal{S}_{\omega, v}$. Very strong nematic order $(S>0.8)$ is achieved for large $\mathcal{P}$ and large $\mathcal{S}_{\omega, v}$. At intermediate values of $\mathcal{P}$ and $\mathcal{S}_{\omega, v}$ the system undergoes an isotropicnematic transition. For a constant Stokes number the transition occurs at $\mathcal{P} \approx 1$. On the other hand for a constant (but large enough) Péclet number, the transition occurs at $\mathcal{S}_{\omega, v} \approx 1$ in the case of Kraichnan flow fields. This shows that these two dimensionless numbers are appropriate to describe the transition and knowledge of both dimensionless parameters is sufficient to predict the degree of alignment of the particles. However, for the point particles in a DNS flow field, the isotropic-nematic transition occurs at a smaller value of $\mathcal{S}_{\omega, v}$.

\subsubsection{Nematic-isotropic transition}

In the previous section different Stokes numbers in the phase diagram were achieved by changing the nematic relaxation constant $\gamma$. However, the Stokes number with 


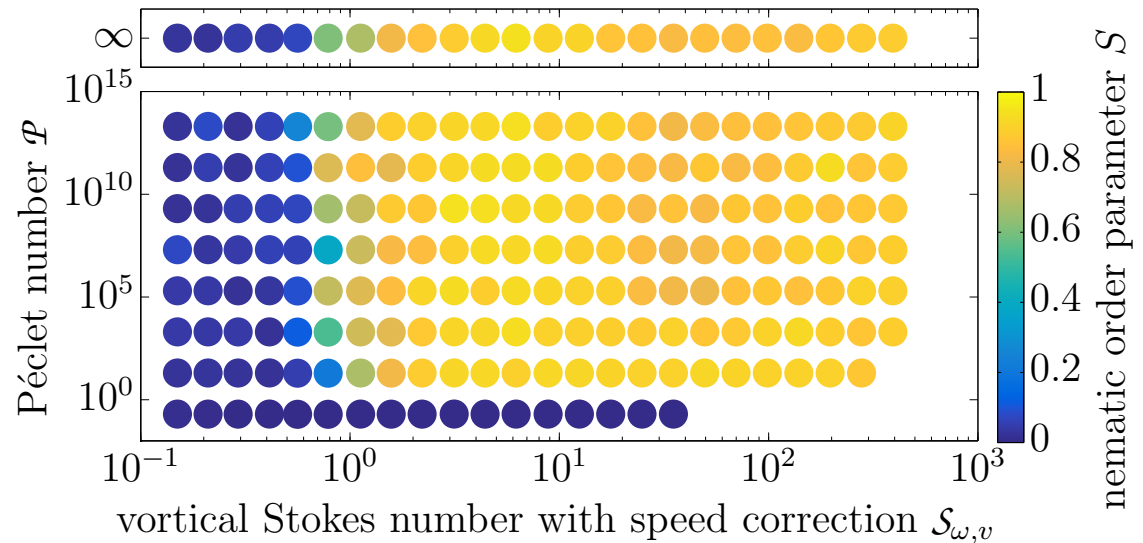

(a) Point particles in Kraichnan flow field

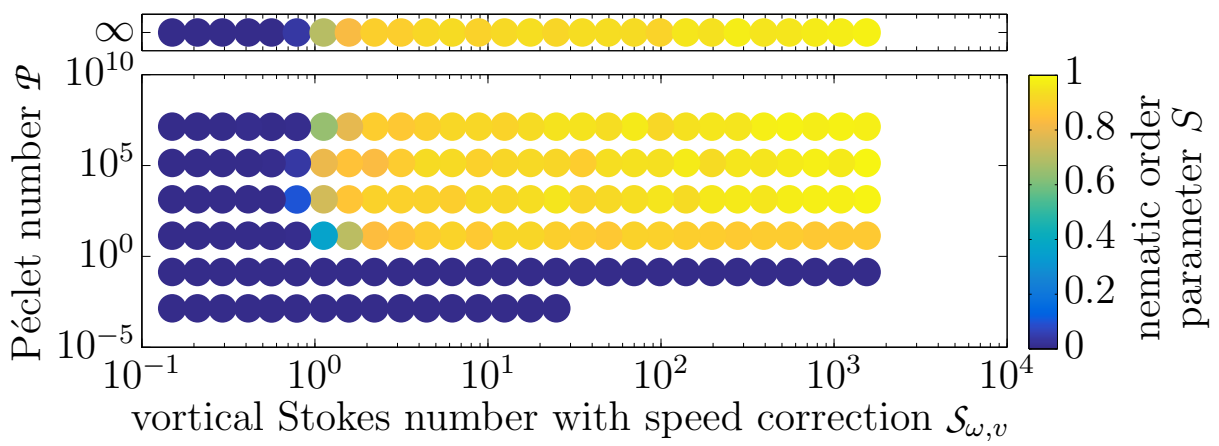

(b) Hard-core particles in Kraichnan flow field

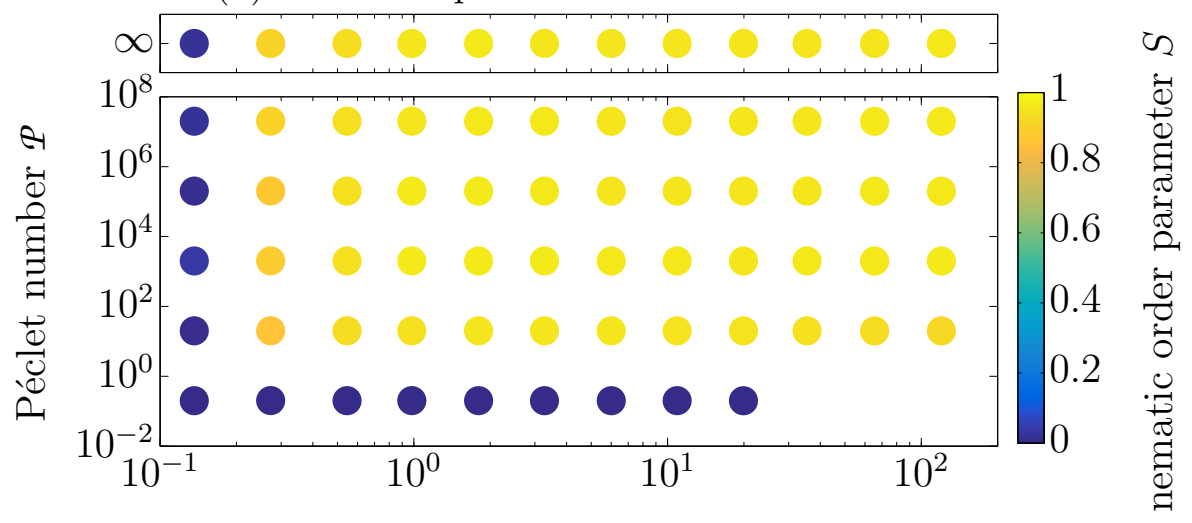

vortical Stokes number with speed correction $\mathcal{S}_{\omega, v}$

(c) Point particles in DNS flow field

Figure 6.1: Nonequilibrium phase diagram of self-propelled particles in a turbulent flow. The nematic order parameter is given in color as a function of Péclet number and vortical Stokes number with speed correction (figures to be published in Breier et al., 2017, supplementary information). 


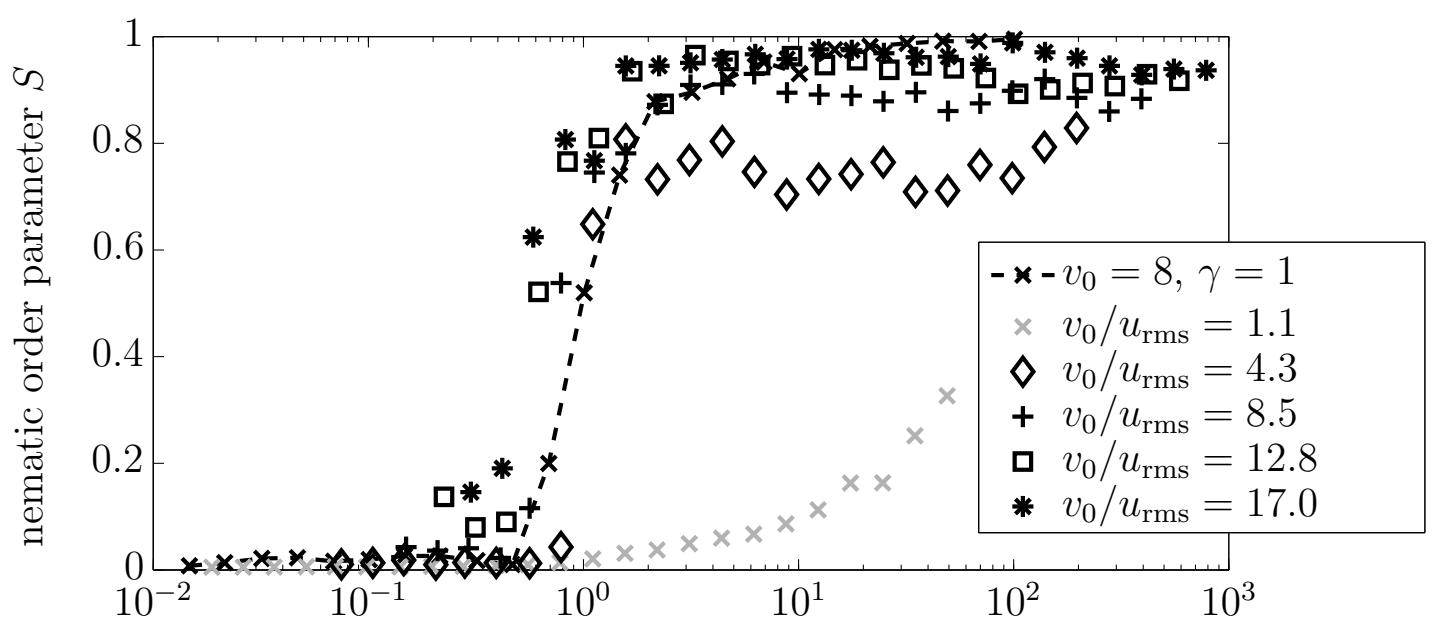

Stokes number with speed correction $\mathcal{S}_{\omega, v}$

Figure 6.2: Nematic order parameter as a function of vortical Stokes number with speed correction. $\mathcal{S}_{\omega, v}$ is varied in different ways: The dashed curve shows the results for constant $v_{0}$ and $\gamma$ with different values of $E_{0}$. The other curves all belong to simulations with the same value of $E_{0}=0.35$ and varying $\gamma$ to change $\mathcal{S}_{\omega, v}$. The difference between these curves are different values of $v_{0}$ as indicated (figure to be published in Breier et al., 2017, supplementary information).

speed correction consists of four parameters in total: the nematic relaxation constant $\gamma$, the self-propulsion speed $v_{0}$, the Kolmogorov shear rate $\omega_{K}$, and the root-meansquare velocity $u_{\text {rms }}$. We will hence independently vary all four parameters to test the robustness of $\mathcal{S}_{\omega, v}$ as the appropriate number to describe the nematic-isotropic transition. All of these simulations are done with point particles in the Kraichnan flow field at a fixed Péclet number $\left(\mathcal{P}=2 \times 10^{3}\right)$. The Kolmogorov shear rate (Eq. 3.20) and $u_{\mathrm{rms}}$ (Eq. 3.19) are coupled via $\omega_{K} \cdot u_{\mathrm{rms}} \propto E_{0}$. Therefore the Stokes number can be varied in three different ways: either $v_{0}, \gamma$, or $E_{0}$ is changed. The results of these simulations are given in Fig. 6.2. It is clearly visible that the isotropic-nematic transition always occurs around $S_{\omega, v} \approx 1$. The only exception is the curve with $v_{0} / u_{\mathrm{rms}}=1.1$. Here the nematic state is not reached in the given range of Stokes numbers. The reason is that $v_{0}$ has to be larger than $u_{\text {rms }}$ for the self-propulsion to have an impact. If the self-propulsion speed is similar to $u_{\mathrm{rms}}$ or even smaller, the particles are effectively passive particles which are advected by the turbulent field. The system would have to be of higher density to reach global nematic order in that case. We can then safely conclude that $\mathcal{S}_{\omega, v}$ serves very well in pinning down the isotropic-nematic transition line under the condition that $v_{0}$ is considerably larger than $u_{\text {rms }}$. 


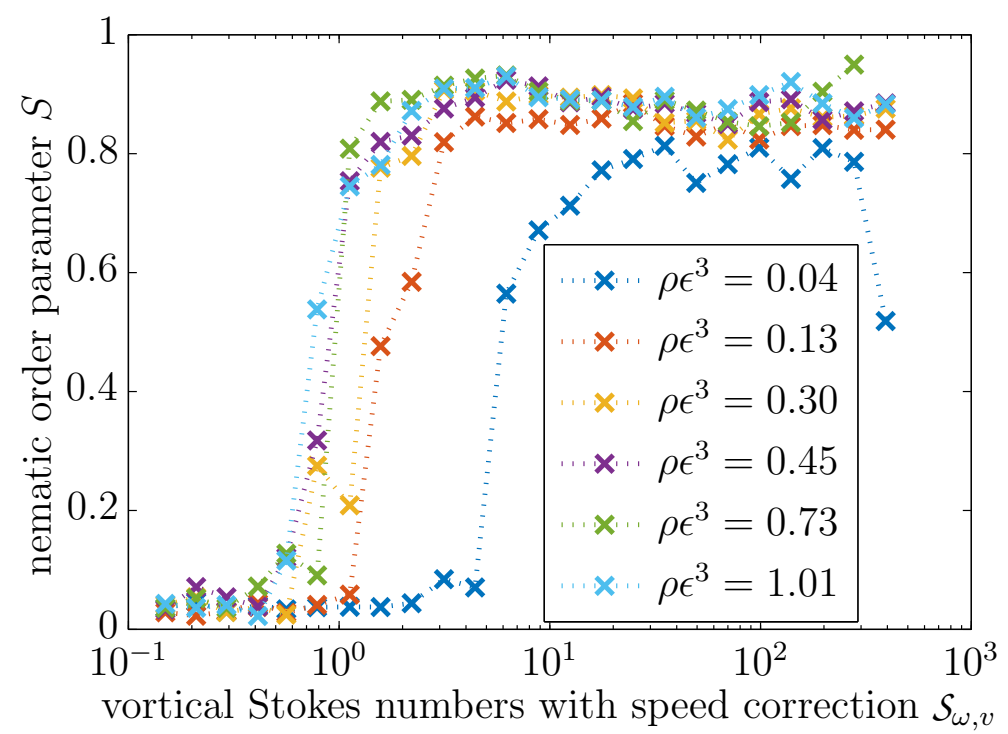

Figure 6.3: Influence of the global number density onto the nematic-isotropic transition of point particles in the Kraichnan flow field. All simulations are performed at an intermediate Péclet number $\left(\mathcal{P}=2 \times 10^{3}\right)$ and different number densities are achieved by varying the number of particles $(1000 \leq N \leq 27000)$ while keeping all other parameters as mentioned before. 
Moreover, we investigate the influence of the global number density onto the isotropic-nematic transition. The results of simulations with different numbers of particles and the same box size (hence, different number densities) are shown in Fig. 6.3. The dimensionless number density $\rho \epsilon^{3}$ varies by almost two orders of magnitude. All curves show a transition from isotropic phase to nematic phase with increasing $\mathcal{S}_{\omega, v}$. The simulations with a number density of $\rho \epsilon^{3}=0.04$ show a drop in nematic order parameter for very high vortical Stokes number. Visual inspection of the corresponding snapshot reveals that the system consists of very dense, polarly ordered clusters. These clusters have a very high local degree of nematic order but do not lead to a high global nematic order. The onset of nematic order $(S>0.3)$ in all curves occurs roughly between $0.7 \leq \mathcal{S}_{\omega, v} \leq 6$. This indicates that there is some variability of the exact position of the isotropic-nematic phase transition with respect to the global density. However, $\mathcal{S}_{\omega, v}$ is still a reasonable measure to know roughly where the transition occurs especially in the given range of number densities with $\rho \epsilon^{3}$ being close to unity.

\subsection{Clustering}

This section includes the quantification of turbulence induced clustering of selfpropelled particles. The three different models (point particles or extended particles in Kraichnan flow field, point particles in DNS flow field) are considered consecutively and the results of different measures are compared.

\subsubsection{Point particles in a Kraichnan flow field}

Figure 6.4 shows the quantification of clustering of point particles in a Kraichnan flow field. For constant Péclet number, the patch concentration enhancement factor $|Q|$ shows a maximum. This "sweet spot" shows that a certain strength of nematic alignment compared to the turbulent vorticity leads to a strong patchiness in the system. On the other hand, for a constant vortical Stokes number $|Q|$ increases as $\mathcal{P}$ increases. In the isotropic phase $\left(\mathcal{S}_{\omega, v}<1\right.$ or $\left.\mathcal{P}<1\right)$, the system never shows enhanced patchiness.

The analysis of number fluctuations shown in Fig. 6.4b reveals that the system undergoes giant number fluctuations. The diagram can be divided in different areas characterized by different values of $\alpha$ : in the isotropic phase, the equilibrium value of number fluctuations $\left(\alpha_{\text {equilibrium }}=0.5\right)$ is found. The largest values of $\alpha$ close to the predicted value for nematic, self-propelled particles $\left(\alpha_{\text {nematic }}=5 / 6\right)$ occur at a transition line surrounding the "sweet spot" as identified by the largest values of $|Q|$ in Fig.6.4a. However, at the "sweet spot" the exponent $\alpha$ takes on values between $\alpha_{\text {equilibrium }}$ and $\alpha_{\text {nematic }}$ indicating that large number fluctuations exist here 
vortical Stokes number with speed correction $\mathcal{S}_{\omega, v}$

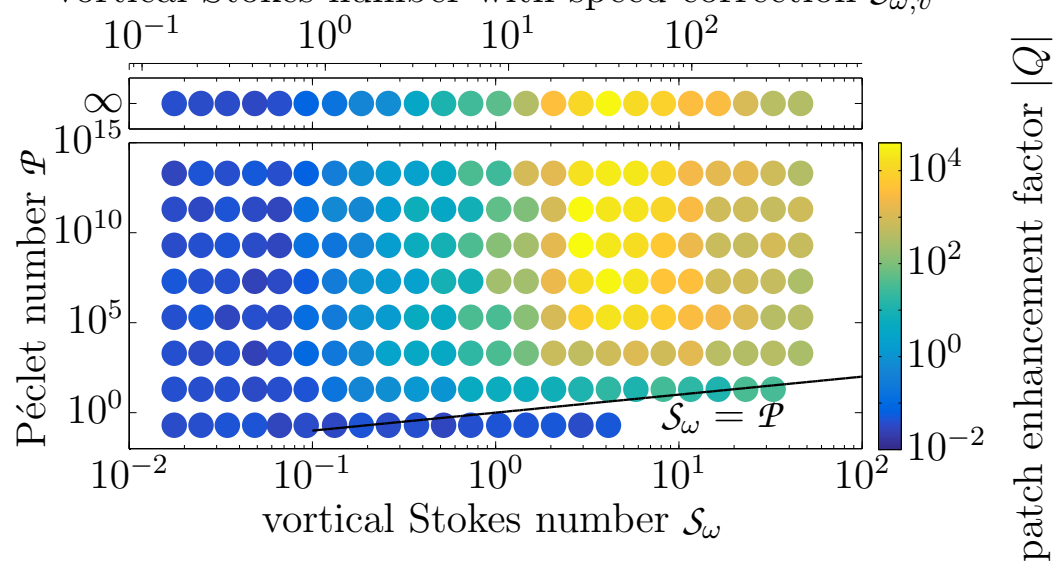

(a) Patch concentration enhancement factor

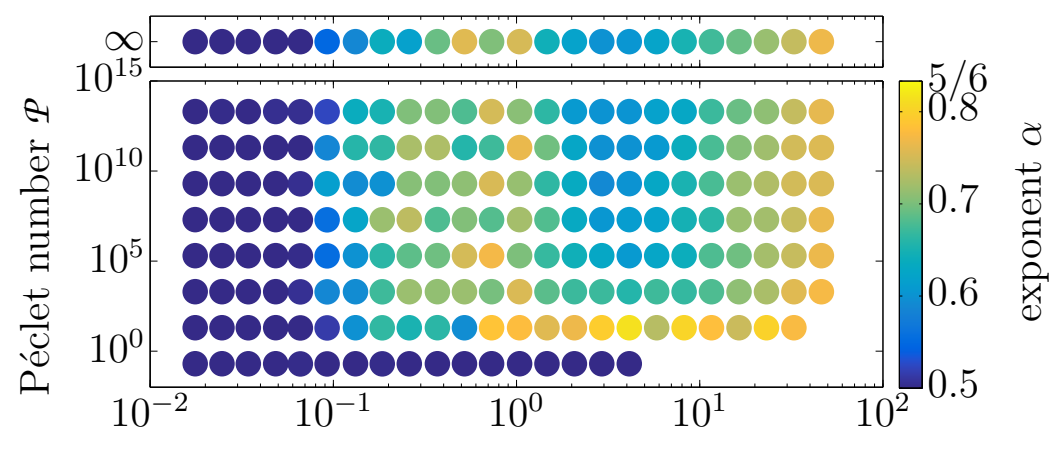

vortical Stokes number $\mathcal{S}_{\omega}$

(b) Exponent of the number fluctuations

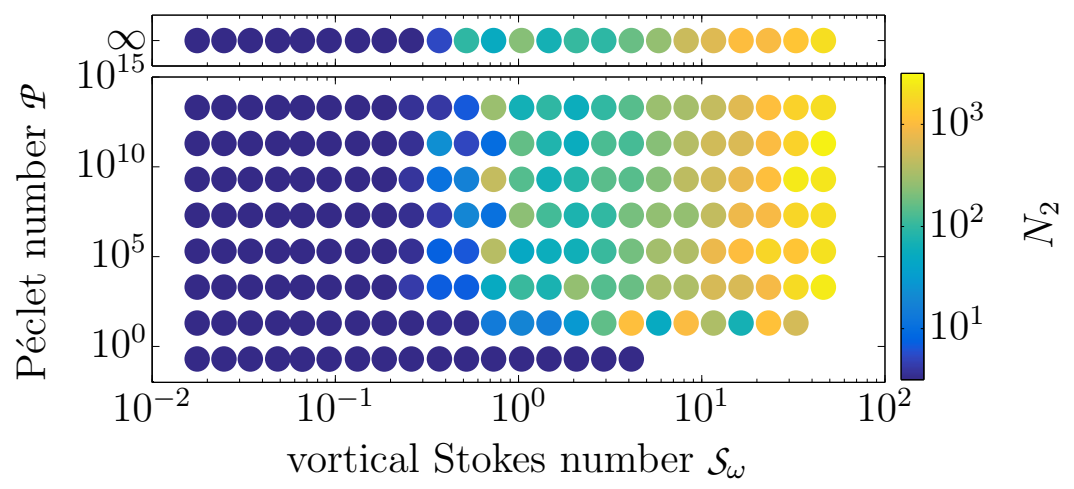

(c) Second moment of the cluster size distribution

Figure 6.4: Clustering of point particles in a Kraichnan flow field. The different measures are shown (in color) as a function of vortical Stokes number and Péclet number. The vortical Stokes number with speed correction is given additionally to clarify the locus of the isotropic-nematic transition line ((a) and (b) to be published in Breier et al., 2017, (b) in supplementary information). 
even though their exponent is smaller than the prediction for nematic particles.

Figure $6.4 \mathrm{c}$ shows a cluster analysis of the same system and exhibits the following behavior: The second moment of the cluster size distribution seems to increase as $\mathcal{S}_{\omega}$ increases. It does not show a maximum or "sweet spot" like $|Q|$ and $\alpha$ do.

To compare the different measures of clustering, we investigate a horizontal cut through the phase diagram at a constant Péclet number $\left(\mathcal{P}=2 \times 10^{13}\right)$. Five steadystate snapshots show the corresponding configurations of representative simulations (see Fig. 6.5). The first snapshot $\left(\mathcal{S}_{\omega}=0.02\right)$ corresponds to an isotropic system and the local density only shows small variations. The corresponding values of $|Q|, \alpha$, and $N_{2}$ are the reference points for a homogeneous system. The snapshot for $\mathcal{S}_{\omega}=0.26$ shows a nematically ordered system which is more or less homogeneous but has larger variations of the local density than the system at $\mathcal{S}_{\omega}=0.02 .|Q|$ indicates a slight increase of patches, while $N_{2}$ is roughly the same as in the isotropic, homogeneous case. However, the resulting value of $\alpha$ implies that the system undergoes giant number fluctuations. The orientations in the snapshot at $\mathcal{S}_{\omega}=0.73$ show global nematic alignment while the distribution of local densities indicates that the particles cluster to some extent (onset of clustering). A dense "stream" is visible as a dense cluster that is self-connected through the periodic boundary conditions. The corresponding patch concentration enhancement factor $|Q|$ suggests that patches are formed but $|Q|$ does not reach its maximum at the corresponding Stokes number. However, both $\alpha$ and $N_{2}$ reach local maxima for the snapshot at $\mathcal{S}_{\omega}=0.73$ which reveals that the system exhibits giant number fluctuations and the cluster size distribution has a rather large variance. The particles in the snapshot at $\mathcal{S}_{\omega}=4.12$ show small dense clusters with local polar alignment. The global alignment is nematic even though it is weaker than in the previous snapshot. $|Q|$ exhibits a maximum while $\alpha$ reaches a local minimum for this system. The number fluctuations are, hence, not as big as before but still larger than the equilibrium fluctuations. Furthermore, $N_{2}$ has an intermediate value and does not exhibit a "sweet spot" at this Stokes number. Finally, the snapshot at $\mathcal{S}_{\omega}=46.12$ shows again global nematic order with distinct polarly aligned clusters. The difference to the previous snapshot is that the individual clusters are larger in size than before. Both $\alpha$ and $N_{2}$ reach their largest values pointing to proper giant number fluctuations as well as a large variance in the cluster size distribution. The patch concentration enhancement factor, however, is smaller than previously but still considerably larger than in the homogeneous case.

In conclusion, one can state that the three measures considered here all describe clustering but they highlight different aspects of the clustering process. First, the patch concentration enhancement factor $|Q|$ highlights by definition the increase of small-scale patches. Upon increasing vortical Stokes number, the system exhibits a transition from a homogeneous distribution of the particles to small patches and finally the patch size increases again. This is reflected in $|Q|$ which exhibits a maximum around $\mathcal{S}_{\omega} \approx 4$. The reason for this maximum and its position will be discussed below. 

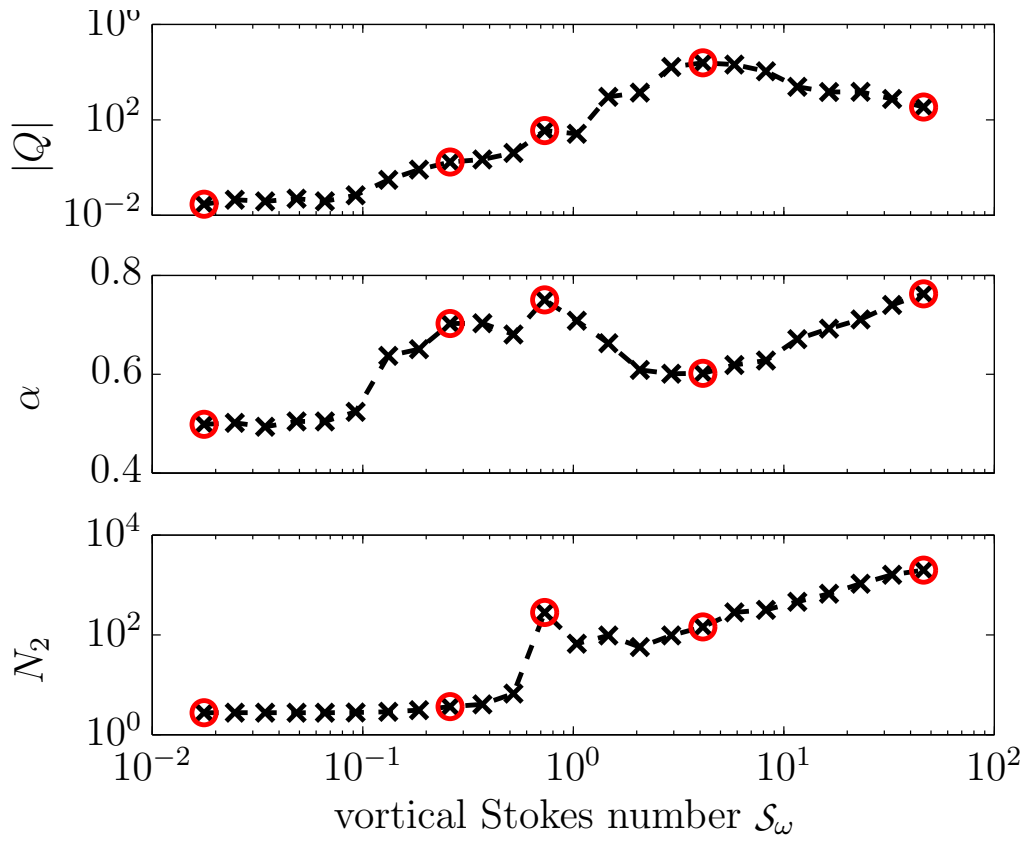

(a) Different measures of clustering for simulations with point particles in Kraichnan flow field $\left(\mathcal{P}=2 \times 10^{13}\right)$ as a function of $\mathcal{S}_{\omega}$. Red circles mark the points for which snapshots are shown in the lower panels.

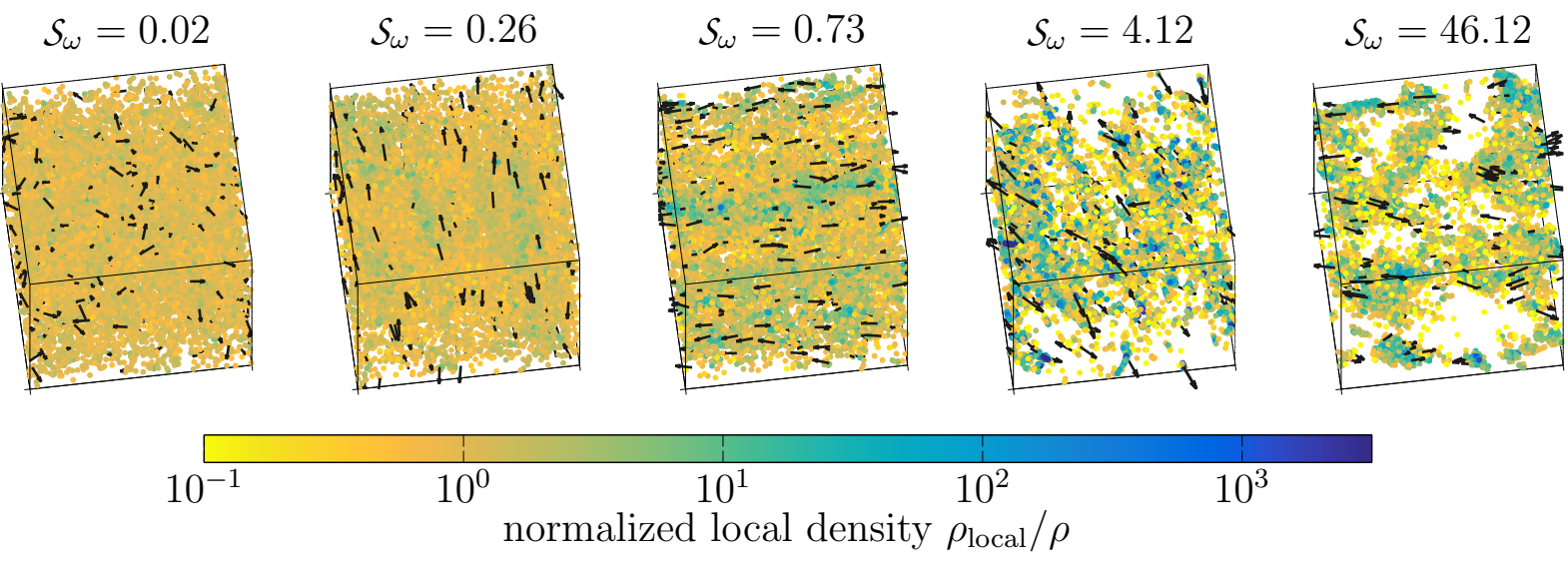

(b) Typical snapshots where $40 \%$ of the particles are plotted as dots. The local density $\rho_{i}=1 / v_{i}$, where $v_{i}$ is the volume of the corresponding Voronoi cell, is given in color. The orientation is given for some example particles.

Figure 6.5: Typical snapshots of point particles in the Kraichnan flow field. 
Second, the analysis of number fluctuations focuses on the degree a given system differs from equilibrium behavior. This difference is very large at the onset of clustering and in the system with vanishing turbulence $\left(S_{\omega} \gg 1\right)$. $\alpha$ does not measure directly how the size of the clusters changes. Third, $N_{2}$ measures the variance of the cluster sizes, i.e. how the size of the clusters varies across the system. The data shows an increase of this variance with increasing $\mathcal{S}_{\omega}$ which means that the cluster distribution gets broader as the influence of the turbulent field through the vorticity decreases. The trend is not fully monotonous but a local maximum of $N_{2}$ occurs at the onset of clustering. In the following, we will focus on $|Q|$ to investigate the increase of small scale patches due to turbulence.

\subsubsection{Extended particles in a Kraichnan flow field}

After having investigated the clustering of point particles in a Kraichnan flow field, we will focus on extended, hard-core particles in a Kraichnan flow field and inspect the differences between the two models to determine the influence of hard cores. Our calculations are shown in Fig. 6.6 in the same manner as it was done for point particles in Fig. 6.4. Again, we compare the results of the three measures for clustering: Patch concentration enhancement factor $|Q|$, exponent $\alpha$ of number fluctuations, and variance of the cluster analysis $N_{2} .|Q|$ is again nearly vanishing in the isotropic phase. For a constant Péclet number, it reaches a maximum as a function of the Stokes number at about $\mathcal{S}_{\omega} \approx 3$. Instead for constant $\mathcal{S}_{\omega},|Q|$ seems to increase with increasing $\mathcal{P}$ and from the given data it is impossible to state whether there is a maximum as well, since the data are rather noisy.

The exponent $\alpha$ of the number fluctuations is equal to the equilibrium value $\alpha_{\text {eq. }}=$ 0.5 in the isotropic phase. In the nematic phase, it increases as both $\mathcal{P}$ and $\mathcal{S}_{\omega}$ increase and does not possess any local maxima or minima. In the given range of Stokes and Péclet numbers it does reach a value similar to the theoretical prediction for nematic $\alpha_{\text {nematic }}=5 / 6$ or even slightly above. This shows that the nematic phase is subject to giant number fluctuations with their strength being a function of Stokes and Péclet number. The variance of the cluster analysis, $N_{2}$, shows a very similar behavior to $\alpha$. It is nearly vanishing in the isotropic phase and grows gradually with growing $\mathcal{P}$ and $\mathcal{S}_{\omega}$ indicating the onset of clustering.

Again, we compare typical snapshots of the system in the steady state to the different values of the clustering measures to understand what kind of clustering occurs. The results in Fig. 6.7 show the curves of $|Q|, \alpha$, and $N_{2}$ as a function of $\mathcal{S}_{\omega}$ for a fixed Péclet number $\left(\mathcal{P}=1.38 \times 10^{7}\right)$. The snapshot at $\mathcal{S}_{\omega}=0.02$ is in the isotropic phase and shows a homogeneous distribution of the particles. Consequently, the corresponding values of all three clustering measures are small, indicating that there are no patches; the system behaves like an equilibrium system, and the cluster analysis has no increase in its variance. 


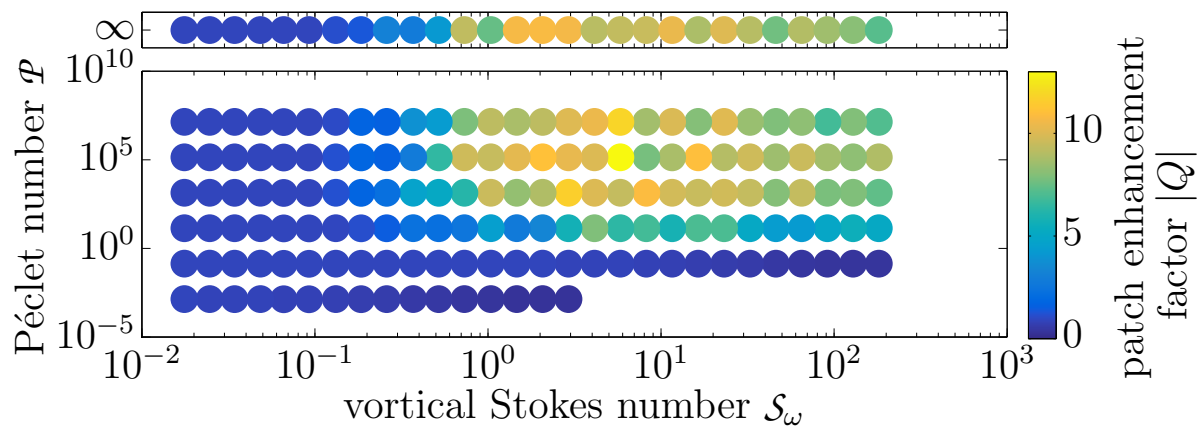

(a) Patch concentration enhancement factor

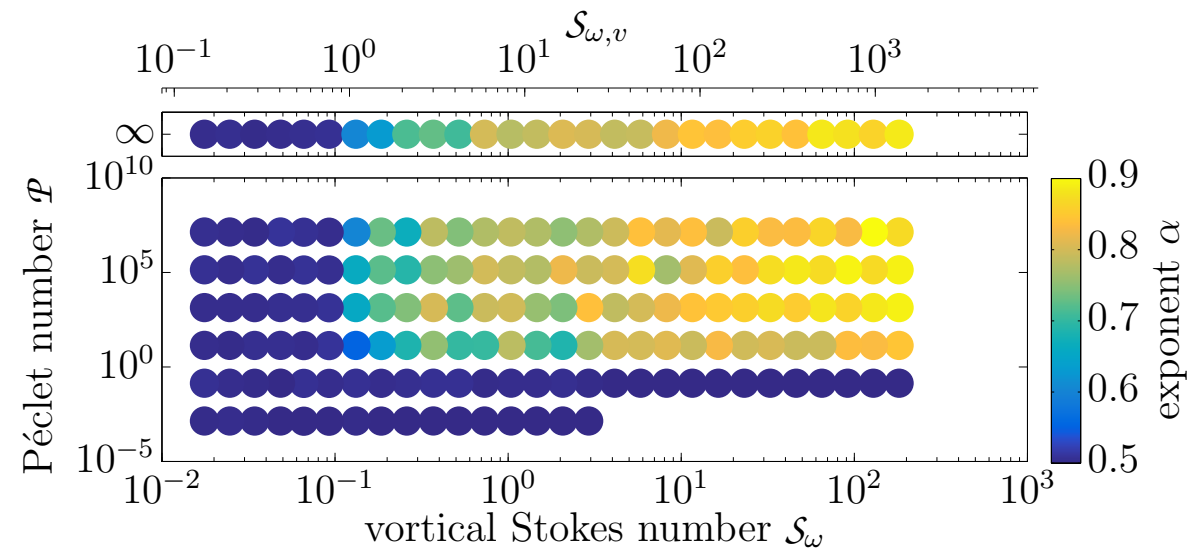

(b) Exponent of analysis of number fluctuations

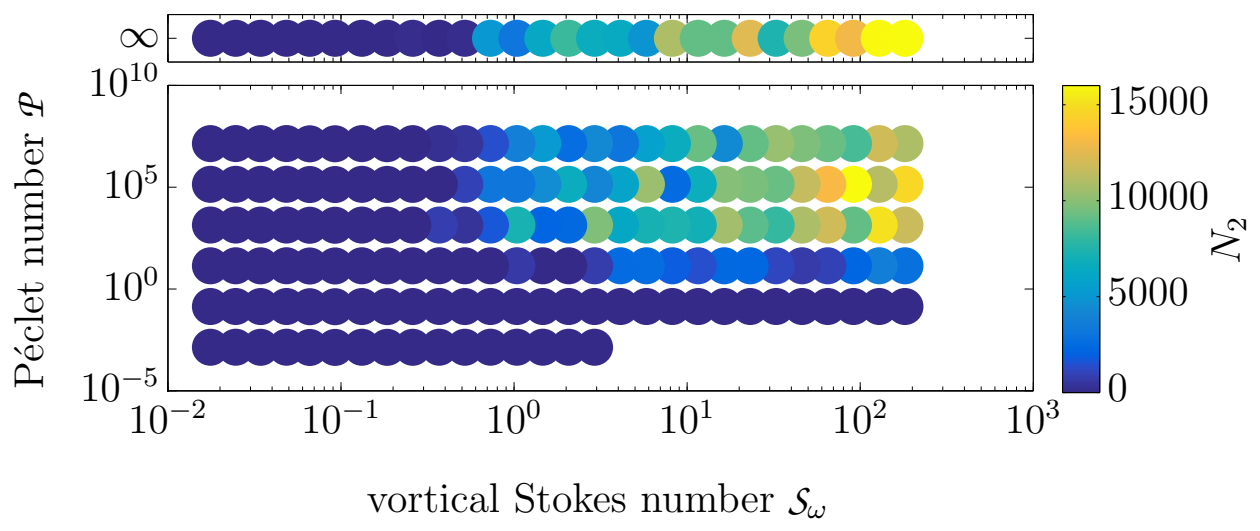

(c) Second moment of cluster size distribution

Figure 6.6: Clustering of hard-core particles in the Kraichnan flow field (analogously to Fig. 6.4) ((a) and (b) to be published in Breier et al., 2017, (b) in supplementary information). 

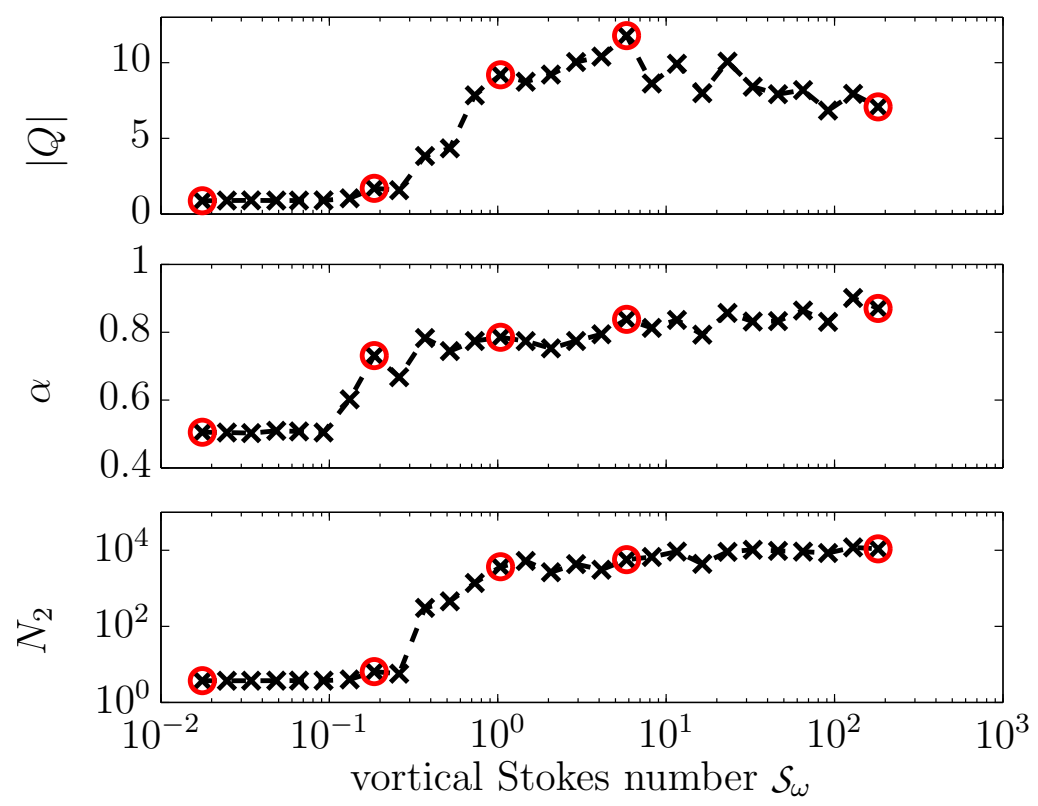

(a) Different measures of clustering as a function of vortical Stokes number for hard-core particles in Kraichnan flow field $\left(\mathcal{P}=1.38 \times 10^{7}\right)$. Red circles mark simulations for which snapshots are shown below.
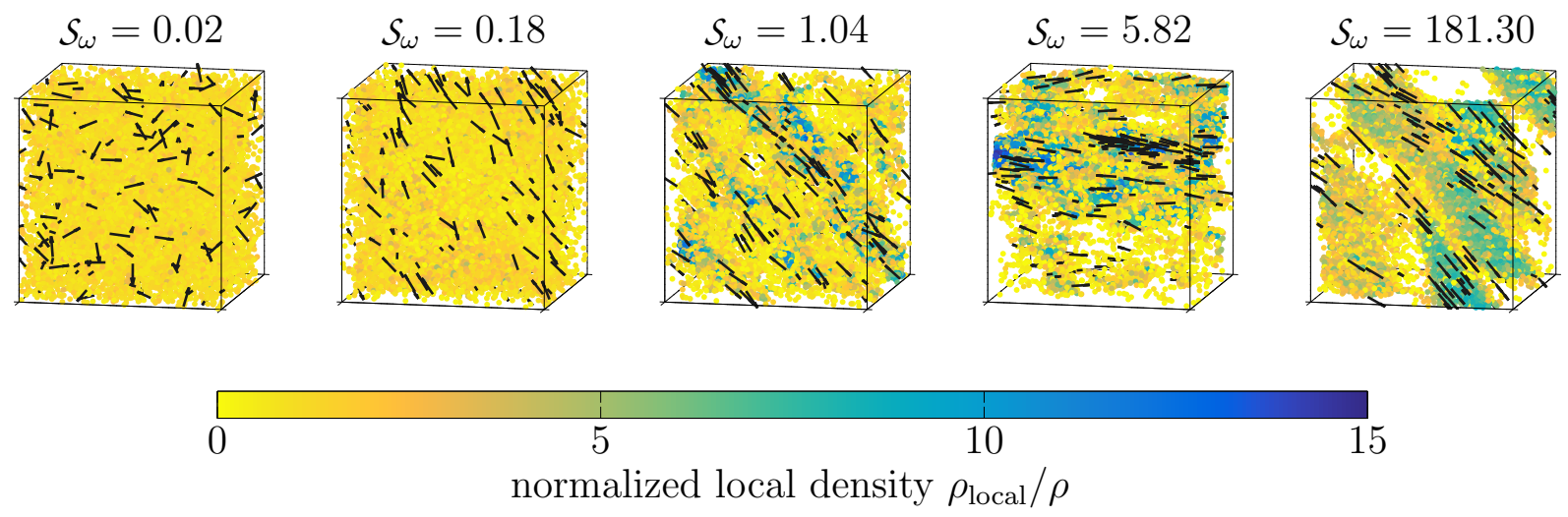

(b) Typical snapshots with $40 \%$ of the particles plotted as dots. The color indicates the local density $\rho_{i}$ as it is calculated from the Voronoi tessellation $\left(\rho_{i}=1 / v_{i}\right)$. A few orientations are given to illustrate the (nematic) order.

Figure 6.7: Typical snapshots of hard-core particles in Kraichnan flow field analogously to Fig. 6.5. 
The snapshot at $\mathcal{S}_{\omega}=0.18$, however, shows global nematic alignment but hardly any changes in the local densities compared to the first snapshot. Hence, both $|Q|$ and $N_{2}$ are at a similar low level. However, the exponent of the number fluctuations is larger than the equilibrium value (but smaller than $\alpha_{\text {nematic }}$ ).

The values of $\alpha$ and $N_{2}$ for $\mathcal{S}_{\omega}=1.04,5.82,181.30$ all exhibit a similarly high level with a slight increase upon growing $\mathcal{S}_{\omega}$. Furthermore, the value of $|Q|$ is much larger than for the homogeneous systems but with a maximum corresponding to the $\mathcal{S}_{\omega}=5.82$ snapshot. The snapshots for $\mathcal{S}_{\omega}=1.04,5.82,181.30$ are similar in that they all show global nematic alignment with variations in the local density. However, the largest values of local density are reached by the system at $\mathcal{S}_{\omega}=5.82$ and also the structure differs to some extent between the three snapshot: The $\mathcal{S}_{\omega}=1.04$ snapshot has only very small voids where no particles are positioned and dense streams are visible which percolate through the periodic boundary conditions. At $\mathcal{S}_{\omega}=5.82$, dense streams also form but they are accompanied by larger voids. Moreover, the local density within the stream is not homogeneous but reaches a maximum in the center of the stream. Finally, the snapshot at $\mathcal{S}_{\omega}=181.30$ reveals similar dense streams which are rather homogeneous in density along and across each stream.

Several differences appear in the comparison of the simulations of point-like and extended, hard-core particles in a Kraichnan flow field as a function of $\mathcal{S}_{\omega}$ (while the behavior as a function of $\mathcal{P}$ does not change drastically). We will discuss in the following these differences as a function of the vortical Stokes number (see Fig. 6.5 for the point particles and Fig. 6.7 for hard cores). The patch concentration enhancement factor $|Q|$ shows similar behavior for both models with an increase from the isotropic to the nematic phase and a "sweet spot" at $\mathcal{S}_{\omega}^{*} \in[3,4]$. However, the values which $|Q|$ reaches in the clustered state are very different: The value for point particles can extend to the order of $10^{4}$ while it is only $10^{1}$ for hard-core particles. The number fluctuations, instead, reveal a completely different behavior if we compare point-like particles with extended ones. The hard cores lead to a monotonously increasing $\alpha$ with $\mathcal{S}_{\omega}$ while the number fluctuations increase for point particles but with a local minimum where small-scale patches form. The results of the variance of the cluster analysis is similar in both models since $N_{2}$ increases with increasing $\mathcal{S}_{\omega}$. The only difference is that in the case of point particles a local maximum is found where a dense stream forms in a rather homogeneous surrounding.

The systems exhibit the same sequence of transitions between collective steadystate organization; they first show a homogeneous and isotropic system, then a homogeneous and nematic system and finally clusters within the nematic order. However, the clusters in the case of hard-core particles always percolate through the periodic boundary conditions while the point particles form much smaller patches. Of course, the hard cores do not allow for the formation of arbitrarily dense clusters (as opposed to point particles), so an investigation of systems of hard cores with lower filling fraction is necessary. 


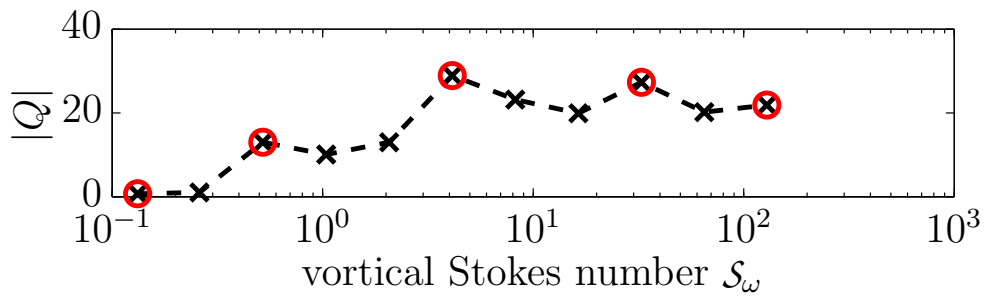

(a) Patch concentration enhancement factor as a function of vortical Stokes number. The red circles denote the simulations which are shown as snapshots below.

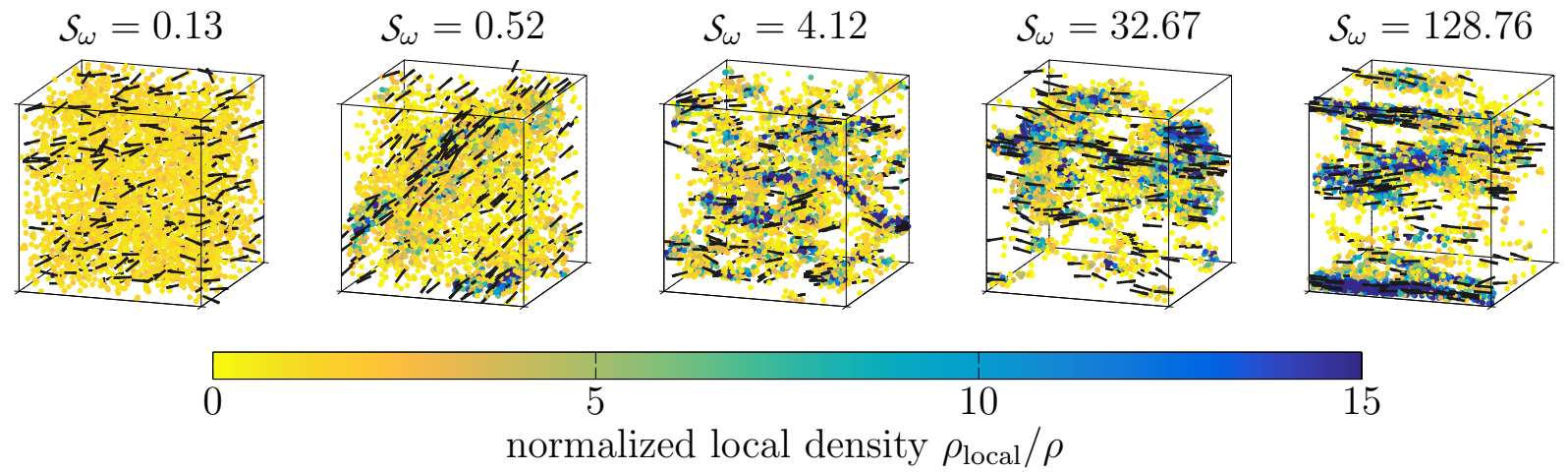

(b) Typical snapshots. Here, only $40 \%$ of the particles are shown as dots with the local density (from Voronoi tesselation) as color. A few orientations are given as small arrows.

Figure 6.8: Extended particles in a Kraichnan flow field at low filling fraction $(N=$ $\left.8000, \phi=0.24 \%, \mathcal{P}=1.38 \times 10^{5}, v_{0} / u_{\mathrm{rms}}=8.5\right)$. 
A lower filling fraction is achieved by keeping the box size fixed and using fewer particles $(N=8000)$. The resulting clustering analysis and characteristic snapshots are shown in Fig. 6.8. All results are within the nematic phase since this is where the clustering occurs. We see a similar behavior of $|Q|\left(S_{\omega}\right)$ as before, namely a maximum around $\mathcal{S}_{\omega} \approx 4$ even though the data are rather noisy. The patch concentration enhancement factor is roughly a factor of two larger than for the hard-core particles at higher filling fraction. It is still roughly three orders of magnitude lower than for point particles, though. All snapshots show nematic order and different degrees of clustering. The particles in the $\mathcal{S}_{\omega}=0.13$ snapshot are homogeneously distributed. In the $S_{\omega}=0.52$ snapshot, a dense stream is formed within a rather homogeneous gas. The particles in the $\mathcal{S}_{\omega}=4.12$ snapshot form small distinct patches with polar alignment. The patches in the $\mathcal{S}_{\omega}=32.67$ snapshot span a larger volume than in the third snapshot, and finally, in the $\mathcal{S}_{\omega}=128.76$ snapshot they percolate through the periodic boundary conditions. The snapshots of hard-core particles at low density are thus more similar to point-particles than to high density hard-core particles as they also show the formation of small-scale clusters.

\subsubsection{Point particles in a DNS flow field}

To validate and justify the use of the Kraichnan flow field, we perform also simulations of point particles which are subject to an external turbulent field which was generated by a DNS (see Section 3.2.3). The resulting patch concentration enhancement factor (Fig. 6.9) shows the same behavior as for point particles in the Kraichnan flow field: $|Q|$ increases from a low value in the isotropic phase with increasing Péclet and Stokes number. Again for constant $P$, it exhibits a "sweet spot": A maximum around $\mathcal{S}_{\omega} \approx 2$. The values of $|Q|$ are of the same order of magnitude as for the point particles in a Kraichnan flow field. Moreover, the analysis of the number fluctuations (Fig. 6.9) exhibits a very similar behavior as compared to the simulations of point particles in a Kraichnan flow field: The exponent $\alpha$ is equal to the equilibrium value in the isotropic phase and intermediate between the equilibrium value and the nematic value in the region of the "sweet spot" in $|Q|$. The transition line between isotropic and larger patchiness is characterized by true giant number fluctuations with $\alpha$ approaching $\alpha_{\text {nematic }}=5 / 6$.

These results show that the Kraichnan flow field is a very good approximation to a turbulent field since it leads to comparable results like state-of-the-art DNSs.

\subsubsection{Temporal evolution of cluster formation}

To understand the formation of the small scale patches in the system, we investigate the temporal evolution of nematic order parameter and patch concentration enhancement factor (Fig. 6.10). The timescales of the two mechanisms nematic order and 
vortical Stokes number with speed correction $\mathcal{S}_{\omega, v}$

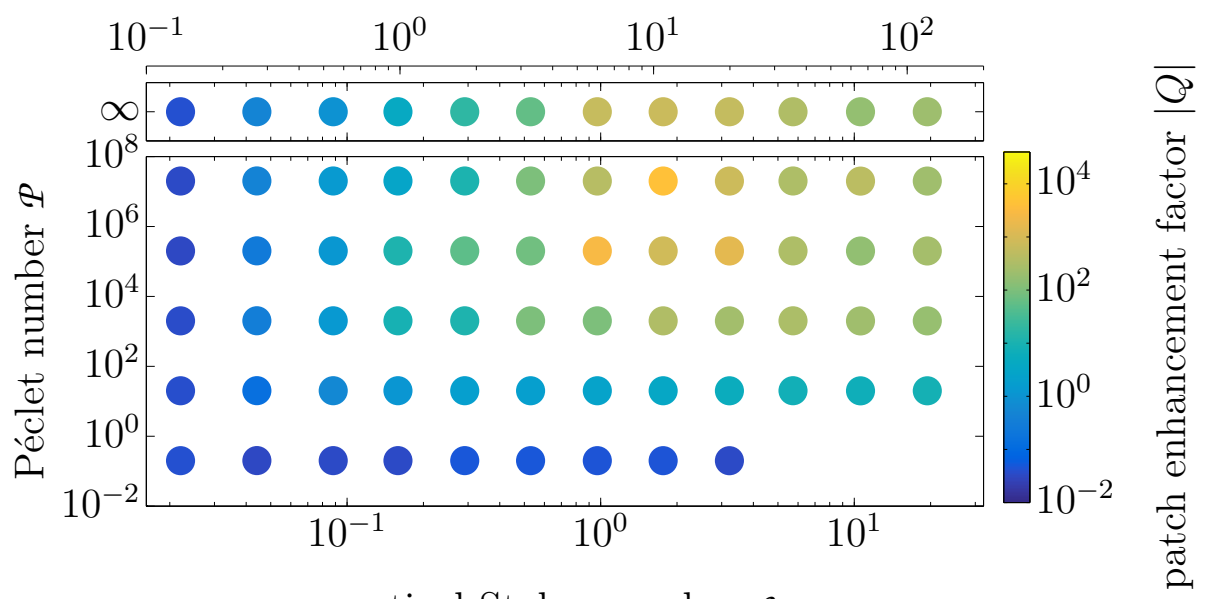

vortical Stokes number $\mathcal{S}_{\omega}$

(a) Patch concentration enhancement factor

vortical Stokes number with speed correction $\mathcal{S}_{\omega, v}$

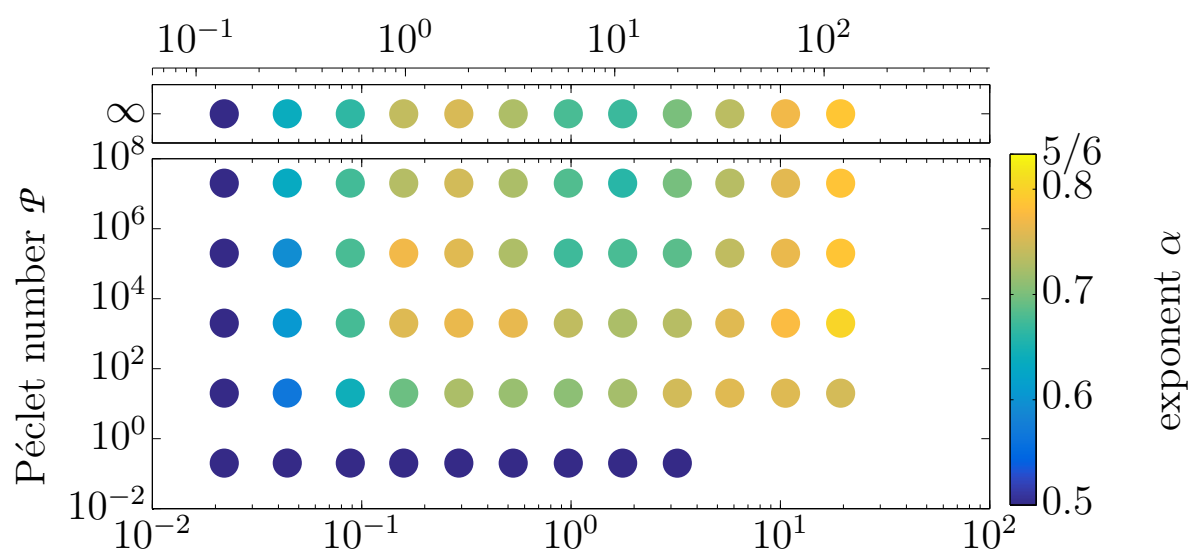

vortical Stokes number $\mathcal{S}_{\omega}$

(b) Exponent of number fluctuations.

Figure 6.9: Clustering of point particles in the DNS flow field as a function of vortical Stokes number and Péclet number ((a) to be published in Breier et al., 2017). 


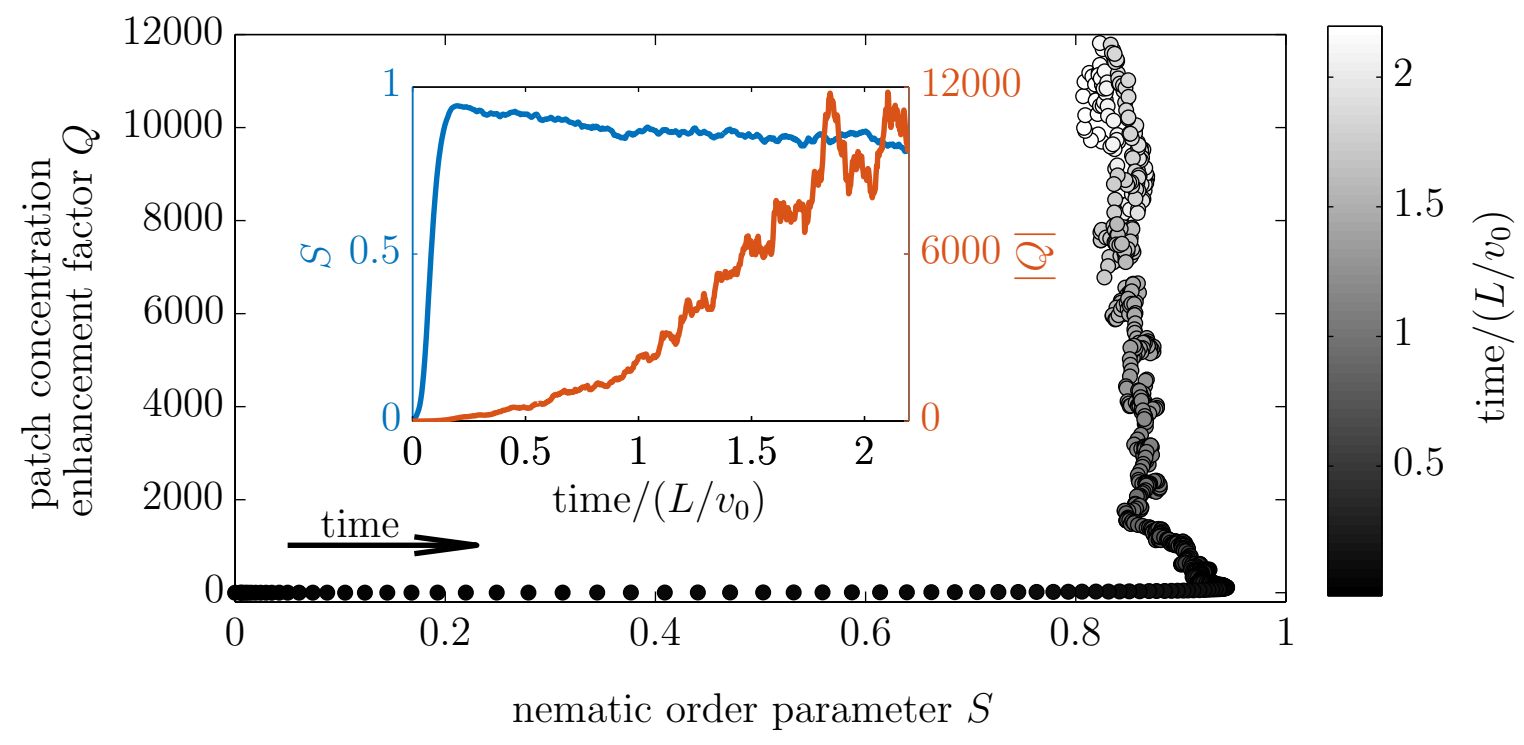

Figure 6.10: Formation of a clustered state: The nematic order parameter and the patch concentration enhancement factor are measured over time for a system which evolves from homogeneous and isotropic to a nematically ordered and clustered state (point particles in Kraichnan flow field, $\mathcal{S}_{\omega}=4.1, \mathcal{P}=2 \times 10^{13}$ ). The main panel shows the temporal evolution of $S$ and $|Q|$, time increases from black to white. The inset shows both $S(t)$ (blue) and $|Q|(t)$ (orange, figure to be published in Breier et al., 2017). 
clustering separate very well: The system first orders nematically $(t<3)$ and then the patch concentration starts to raise significantly $(t>10)$. Moreover, the global nematic order parameter decreases slightly when the patches are forming. This separation of time scales together with the vortical Stokes number already points to the importance of vorticity in the formation of the small-scale clusters. The Stokes number can be understood as the ratio of the time scale of vorticity to the time scale of nematic alignment. The maximum clustering is found where this ratio is larger than one but not too large $\left(S_{\omega}<10\right)$ and the two time scales are thus separated. Hence, our hypothesis is that the clusters form due to the interplay of nematic alignment and turbulent vorticity.

\subsubsection{Influence of integral length scale}

From all our results on clustering (Figs. 6.4, 6.6, and 6.9), we see that the vortical Stokes number $\mathcal{S}_{\omega}$ is the appropriate dimensionless parameter to describe the onset of small scale patches. The maximum value of $|Q|$ is found for $\mathcal{S}_{\omega} \in[2,4]$. The Stokes number is varied either by changing the nematic relaxation constant or the Kolmogorov shear rate. Hence, the vorticity of the turbulent field is important for the formation of clusters. Moreover, we learn from the temporal evolution of the cluster formation (Fig. 6.10) as well as from the phase diagrams (Fig. 6.1) that nematic order is necessary. Thus, the two length scales involved in the clustering are the range of nematic interaction $\epsilon$ and the integral length scale of the vorticity $L_{11}^{\omega}$ (Eq. 3.21b) which characterizes the turbulent field. The influence of $L_{11}^{\omega}$ can be studied by changing the maximum and minimum wavelengths in the system while keeping the ratio of both length scales fixed to ensure the same Taylor-based Reynolds number (Eq. 3.30).

The results of simulations for point particles in a Kraichnan flow field with different ratios $L_{11}^{\omega} / \epsilon$ and no stochastic noise $(\mathcal{P} \rightarrow \infty)$ are given in Fig. $6.11\left(0.23 \leq L_{11}^{\omega} / \epsilon \leq\right.$ 3.43). The nematic order parameter (Fig. 6.11a) shows that changing the integral length scale does not affect the isotropic-nematic transition which occurs around $S_{\omega, v} \approx 1$ for all simulations. However, the value of the nematic order parameter for large $\mathcal{S}_{\omega, v}$ increases as $L_{11}^{\omega} / \epsilon$ decreases. The patch concentration enhancement factor (Fig. 6.11b) increases with increasing vortical Stokes number for all ratios $L_{11}^{\omega} / \epsilon$. Moreover, all curves collapse for $\mathcal{S}_{\omega}<10^{-1}$ which is in the isotropic phase where the turbulent field dominates over the nematic alignment. However, upon closer inspection, the curves $|Q|\left(S_{\omega}\right)$ separate into two sets: All simulations with $L_{11}^{\omega} / \epsilon>0.25$ show a prominent and rather sharp peak around $\mathcal{S}_{\omega} \approx 3$. In contrast, the simulations with $L_{11}^{\omega} / \epsilon<0.25$ do not show a peak but rather a plateau for large Stokes numbers. All curves of $|Q|\left(S_{\omega}\right)$ seem to approach one single value of the patch concentration enhancement factor for very large $\mathcal{S}_{\omega}: Q^{*}$. This is the regime where nematic order dominates over the turbulent vorticity. The value of $Q^{*}$ can be understood by investigating the patchiness of the non-turbulent system. 


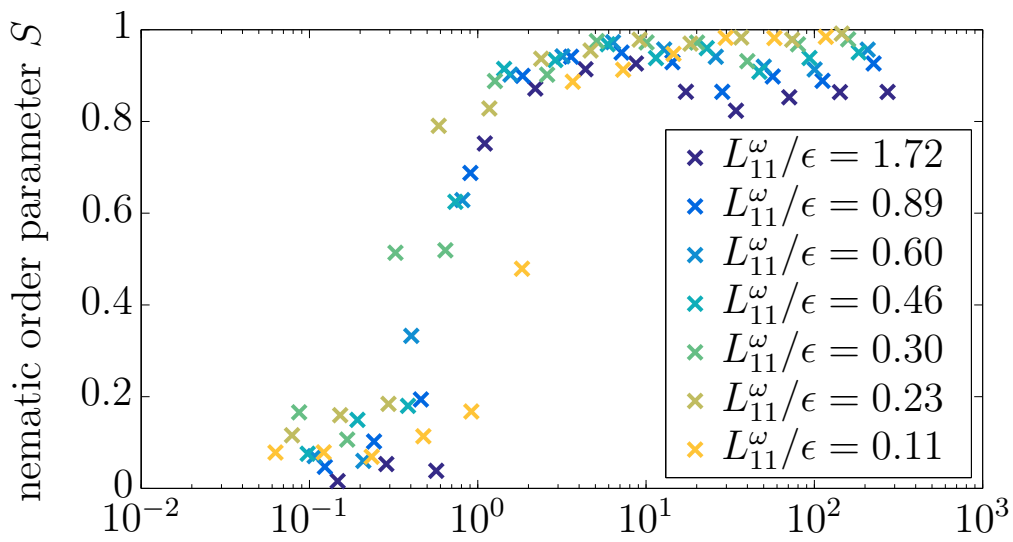

vortical Stokes number with speed correction $S_{\omega, v}$

(a) Nematic order parameter as a function of vortical Stokes number with speed correction.

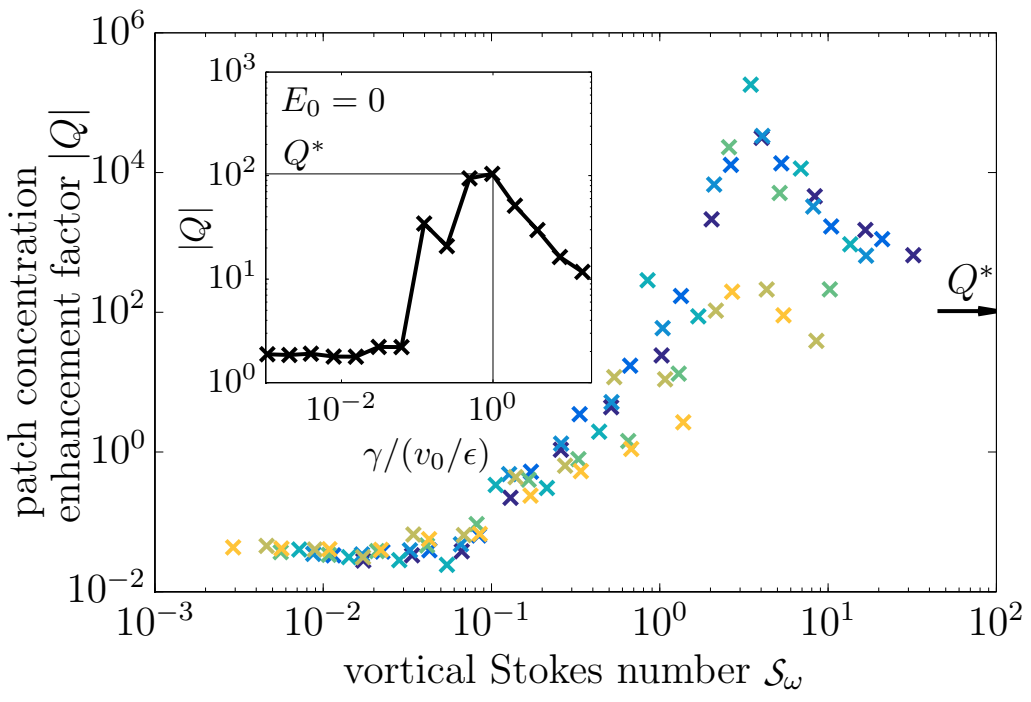

(b) Patch concentration enhancement factor as a function of vortical Stokes number. The inset shows $|Q|$ as a function of $\gamma /\left(v_{0} / \epsilon\right)$ for simulations with $\mathcal{P} \rightarrow \infty$ and $E_{0}=0$ (hence $\left.S_{\omega} \rightarrow \infty\right) . \quad Q^{*}$ is the value of $|Q|$ at $\gamma=v_{0} / \epsilon$ for a simulation with infinite Péclet and vortical Stokes numbers.

Figure 6.11: Influence of the integral length scale of the vorticity on the isotropicnematic transition and on the clustering (point particles in Kraichnan flow field). The Péclet number of all simulations is infinite $(\eta=0)$. Different colors indicate different integral length scales ((b) to be published in Breier et al., 2017). 


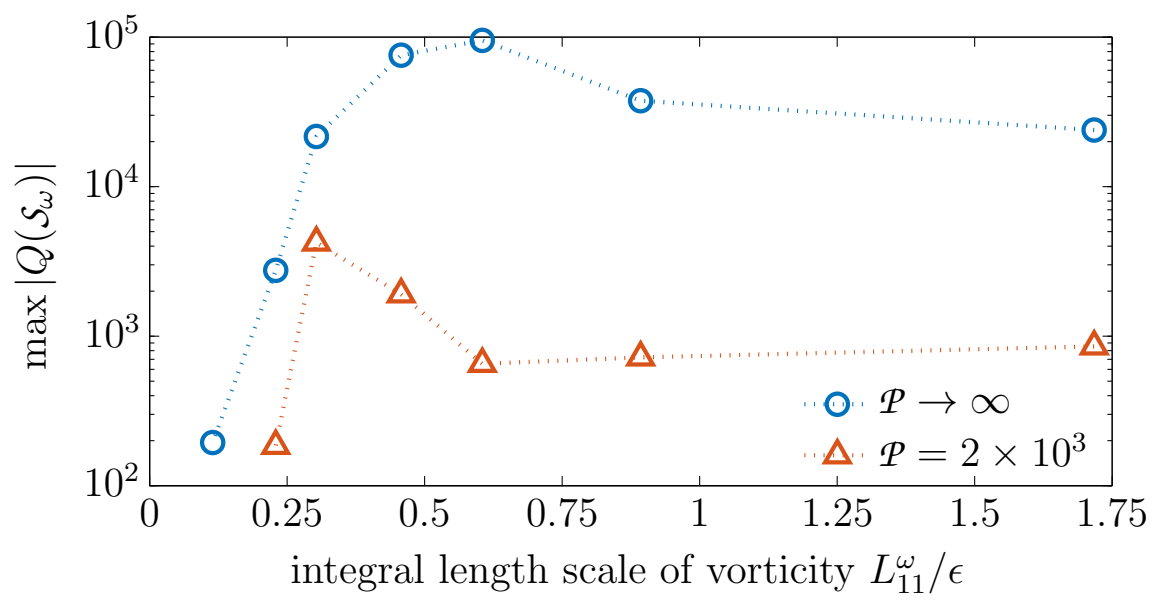

Figure 6.12: Maximum patch concentration enhancement factor as a function of integral length scale. The two curves belong to simulations with different $\mathcal{P}$ as indicated. The curve with $\mathcal{P} \rightarrow \infty$ is calculated from 40 independent simulations per value of $\mathcal{S}_{\omega}$ (as in Fig. 6.11) in the vicinity of the maximum of $|Q|$ while for the curve with $\mathcal{P}=2 \times 10^{3}$ only one individual simulation has been taken into account (to be published in Breier et al., 2017).

The system without stochastic noise and without turbulence $\left(E_{0}=0\right.$ and hence $\left.S_{\omega} \rightarrow \infty\right)$ is only a function of three parameters: the nematic relaxation constant $\gamma$, the self-propulsion speed $v_{0}$, and the nematic interaction range $\epsilon$. These three parameters form a dimensionless number $\gamma /\left(v_{0} / \epsilon\right)$ which compares the time scale of self-propulsion with the time scale of the nematic alignment. $|Q|$ exhibits a maximum where the two time scales are equal $\left(\gamma=v_{0} / \epsilon\right)$ and the value it assumes there is denoted by $Q^{*}$. This value matches in magnitude the value which all curves $|Q|\left(S_{\omega}\right)$ seem to approach for large Stokes numbers.

The existence of a peak in $|Q|$ hence depends on the ratio of the integral length scale of vorticity to the nematic interaction range. We now want to investigate the hypothesis that the most small patches (largest value of $|Q|$ ) form when both length scales are equal. In Fig. 6.12 we plot the maximum of $|Q|$ as a function of $L_{11}^{\omega} / \epsilon$. The curve for $\mathcal{P} \rightarrow \infty$ exhibits a clear maximum where the integral length scale of vorticity is half of the nematic interaction range. The same analysis was done for simulations with a finite Péclet number resulting in lower patch concentration enhancement factors but a similar behavior. Nevertheless, we can safely conclude that the strongest formation of small-scale patches occurs when the integral length scale of the vorticity and half of the nematic interaction range of the SPPs are equal. 


\subsubsection{Influence of Reynolds number}

Up to this point, all simulations were done for a constant Taylor-based Reynolds number $\mathcal{R}_{\lambda} \approx 48$. In this subsection we intend to investigate the influence of the Reynolds number onto the nematic-isotropic transition and onto the formation of patches. All simulations presented here are for point-like particles in a Kraichnan flow field with number density $\rho \epsilon^{3}=1$ and varying numbers of particles from $30^{3}$ to $100^{3}$. Hence, the minimum wavenumber (being bound by the box size) varies while the maximum wavenumber is $k_{\max }=30$ for all simulations. The smallest length scale in the turbulent field is thus the same for all simulations. However, the resulting integral length scale of the vorticity is not the same for all simulations but necessarily increases slightly in the range $1.03 \leq L_{11}^{\omega} / \epsilon \leq 1.19$ because the integral length scale is a function of the ratio of the maximum to the minimum wavenumber (see Eq. 3.21b). This interval of integral length scales is well within the range where we expect an enhanced patch concentration due to the turbulent field as discussed in the previous Section 6.2.5. We do not expect this small change to have an effect onto the results. The nematic-isotropic transition and the clustering of these simulations with varying Reynolds number are shown in Fig. 6.13. The curves of the nematic order parameter collapse for small vortical Stokes numbers with speed correction, i.e. in the isotropic phase. The isotropic-nematic transition occurs around the same vortical Stokes number and all simulations reach a similarly high global nematic order parameter in the nematic phase. However, the data suggest that the value of $S$ deep in the nematic phase decreases with increasing Reynolds number. The curves of $|Q|$, on the other hand, all collapse within their accuracy for high vortical Stokes numbers and all show the "sweet spot" where small patches are observed. But the degree of clustering measured by $|Q|$ in the isotropic phase decreases with increasing turbulent Reynolds number. To conclude, the main finding, which is the "sweet spot" in $|Q|$, is preserved in the investigated range of turbulent Reynolds numbers.

\subsection{Discussion}

The nonequilibrium phase diagrams of all three models show two distinct phases: An isotropic phase and a nematic phase. The isotropic phase is present in two domains of the phase diagram $\left(\mathcal{P}<1\right.$ and for small $\left.\mathcal{S}_{\omega, v}\right)$ because there exist two different and independent mechanisms to destroy nematic order in the model. The system cannot develop nematic order if the stochastic noise is dominant in comparison to the strength of the nematic alignment. Even if two particles meet and tend to align in one time step, their orientations will be effectively randomized by the stochastic noise in the next time step. The second mechanism which prevents the system from globally aligning is the turbulent field. If the vorticity of the turbulent field is larger than the 


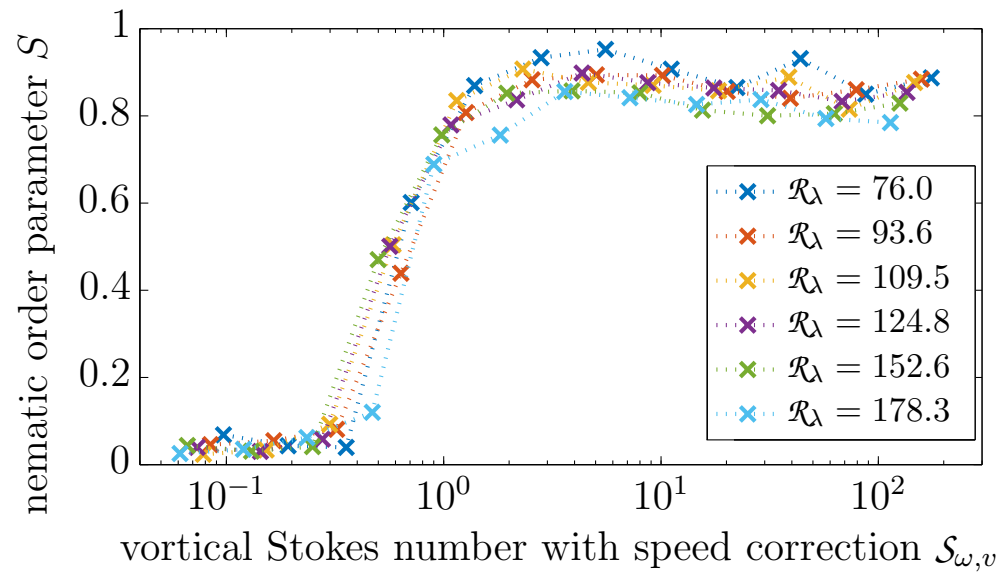

(a) Isotropic-nematic transition.

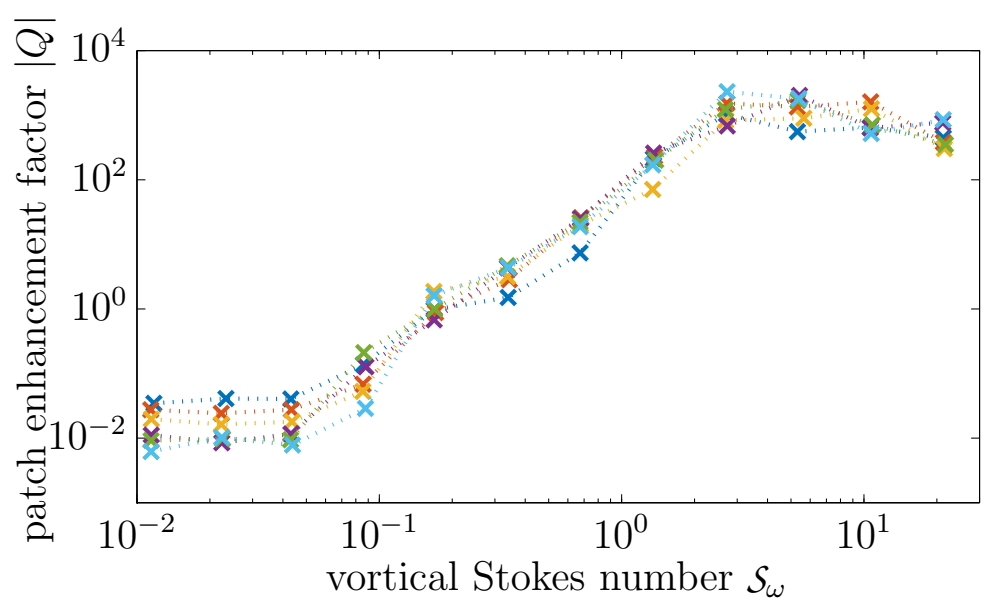

(b) Patch concentration enhancement factor.

Figure 6.13: Influence of Taylor-based Reynolds number on simulations with point particles in the Kraichnan flow field (to be published in Breier et al., 2017, supplementary information). 


\begin{tabular}{lll}
\hline Parameter & Kraichnan flow & DNS flow \\
\hline $\mathcal{R}_{\mathrm{\lambda}}$ & 48 & 17 \\
& $k_{\max } / k_{\min }=16$ & $L_{11} / \eta_{K}=10.8$ \\
$\omega_{K}$ & 4.54 & 4.53 \\
$u_{\mathrm{rms}}$ & 0.94 & 1.29 \\
& $2 \pi / k_{\max } / \epsilon=1.86$ & $\eta_{K} / \epsilon=0.30$ \\
\hline
\end{tabular}

Table 6.2: Comparison between flow fields from DNSs and kinematic simulations (using the standard parameters).

nematic relaxation constant, the turbulent field acts in the same way as a noise and effectively randomizes the orientations of the particles. The difference between the turbulent field and the true stochastic noise is that the turbulent field is correlated in time and space. The critical vortical Stokes number with speed correction for the isotropic-nematic transition is $\mathcal{S}_{\omega, v}^{\text {crit }}=1$ for the particles (point-like or extended) in the Kraichnan flow field. However, it is rather $\mathcal{S}_{\omega, v}^{\text {crit }}=0.2$ for the point particles in the DNS flow field. The main parameters, which characterize the flow fields, are given in Tab. 6.2. They match roughly in Kolmogorov shear rate and root-mean-square velocity. The Taylor-based Reynolds numbers of the two approaches are comparable; the kinematic simulations are performed at a larger $\mathcal{R}_{\lambda}$, though. A direct comparison between the two has to be taken with a grain of salt, because the definition of $\mathcal{R}_{\lambda}$ in a Kraichnan flow field is difficult (see discussion in Section 3.1.4). However, the ratio between the largest and smallest length scales in the two simulation approaches is of the same order of magnitude which makes them comparable. Nevertheless, the ratio between smallest length scale of the turbulent field and nematic interaction range differs substantially. For the Kraichnan flow field the smallest wavelength is almost twice as large as the nematic interaction range. In contrary, the Kolmogorov length scale $\eta_{K}$ of the DNS flow field is only a third of the nematic interaction range. The Kolmogorov length scale serves to estimate the size of the smallest structures in the flow field. However, this difference cannot explain why the isotropic-nematic transition occurs at a value of $\mathcal{S}_{\omega, v}$ smaller than unity when a DNS flow field is used, because a smaller Kolmogorov length scale should rather destroy than stabilize nematic order.

All three models show a comparable behavior of the clustering as measured by $|Q|$. The clustering of particles is clearly enhanced in the nematic phase of our system as compared to the isotropic phase. Moreover, the clustering shows a non-monotonic behavior when the Stokes number is increased. Hence, a specific ratio between turbulent vorticity and nematic interaction strength favors the formation of small patches. To understand this behavior, we discuss the formation of clusters. We know that we need nematic interaction for the cluster formation since clusters are only forming in the ne- 
matic phase. Moreover, the self-propulsion is important because non-motile particles would just act as passive tracers. The turbulent flow field is incompressible and hence volume-preserving. This means that the particles will always remain homogeneously distributed, if they followed a homogeneous distribution at one point in time. All presented simulations start from a homogeneous distribution of particles and hence the self-propulsion is necessary to break the volume conservation and form clusters. The temporal evolution of the clustering system shows that the system firstly orders nematically and only then the clusters are forming. The mechanism is the following: In the beginning of a simulation all particles are randomly distributed with random orientations. They move forward due to their self-propulsion and are advected by the turbulent field which simply acts as a perturbation of their self-propulsion velocity in this initial phase. Moreover, their orientations are subject to stochastic noise and turbulent vorticity where also the latter acts in this early stage as an additional noise. If the time scale of the nematic interaction is smaller than the time scale of rotational diffusion $\left(1 / \gamma<1 / D_{r}\right)$, the particles can order nematically rather quickly. This condition is equal to $\mathcal{P}>1$. Moreover, also the turbulent field must not be stronger than the nematic interaction which is equal to the condition $S_{\omega, v}>1$. Once the particles are ordered nematically, their orientations are altered by the turbulent vorticity and the stochastic noise. We assume the turbulent vorticity to be larger than the stochastic noise $\left(S_{\omega}<P\right)$ to be able to neglect the stochastic noise and focus on the influence of the turbulent field. This assumption is necessary to study the effect of the turbulent field and corresponds to the area in the $|Q|\left(\mathcal{S}_{\omega}, \mathcal{P}\right)$-plot above the black line (see Fig. 6.4a). So the nematically ordered particles are moving into the direction of their intrinsic orientations and are advected by the turbulent field. Moreover, the turbulent vorticity alters their orientations and makes them different from the orientations of the neighboring particles. This alteration thus increases the probability of a particle to bump into another particle. If particles meet, they align. They can form (the beginning of) a cluster if they align polarly and they are subject to roughly the same turbulent field so that the turbulent field does not tear them apart. This last condition is quantified by the integral length scale of the turbulent field. We showed that the integral length scale of the vorticity has to be larger than a quarter of the nematic interaction for a "sweet spot" in the clustering to occur. If the integral length scale is smaller, the structures of the turbulent field are large enough so that the particles will be effectively torn apart by the turbulent vorticity. The clustering is always enhanced in the nematic phase as compared to the isotropic phase. This enhancement is due to the fact that the nematic order helps particles to stay close to each other. However, if the integral length scale of the vorticity is larger than a quarter of the nematic interaction range, the strongest clustering should occur if the relevant time scale of the turbulent field and the time scale of the nematic interaction match. The relevant time scale of the turbulent field is the Kolmogorov 


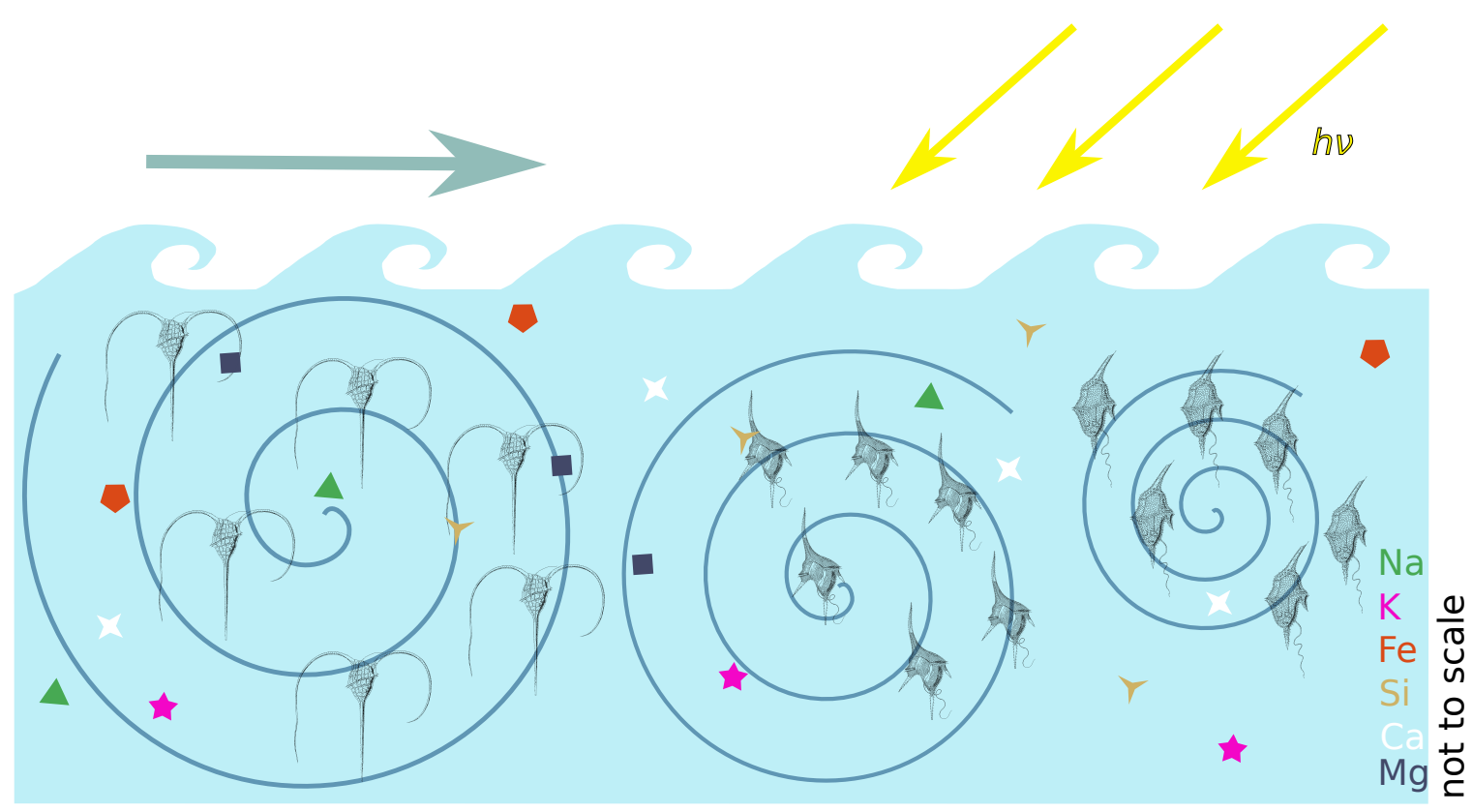

Figure 6.14: Illustration of the consequence of turbulence induced clustering on different phytoplankton species (cf. Fig. 1.4). The images of the species have been adapted from Haeckel (1899, online: http://biolib.mpipz.mpg.de/haeckel/kunstformen/ natur.html, copyright 1999 Kurt Stueber und Max-Planck-Institut für Züchtungsforschung).

time scale $\tau_{\eta}=\sqrt{\nu / \epsilon}$ which is related via $\epsilon=2 \nu \int k^{2} E(k) \mathrm{d} k$ to the Kolmogorov shear rate as $\tau_{\eta}=1 / \omega_{K}$. A vortical Stokes number $\mathcal{S}_{\omega}^{c}=2$ hence means that the two times equal and this is where the maximum in $|Q|$ was found. To conclude, this means that turbulence can increase the small patches of the system if the integral length scale of the vorticity is larger than a quarter of the nematic interaction range. The maximum increase is found where the Kolmogorov time scale matches the time scale of the nematic alignment. This precisely matches the results of Wang and Maxey (1993) who found that "the strongest accumulation happens when the particle response time is comparable to the Kolmogorov timescale" in a system of settling spherical particles in homogeneous turbulence. More recently, a maximum in clustering of gyrotactic particle in turbulence was also found by Durham et al. (2013) when the Kolmogorov time scale and the typical reorientation time of the particles are equal. This balance of time scales seems to be universal to all systems of particles which are subject to a turbulent field.

The hydrodynamic interaction of planktonic microorganisms is to first order nematic (see Section 2.1 and Baskaran and Marchetti, 2009). If they exist in a turbulent environment, the maximum clustering will occur where twice the integral length scale 
of the turbulent field matches the nematic interaction range. In principle the nematic interaction range is just a model parameter in our system of self-propelled particles. Our model is justified by the symmetry of hydrodynamically interacting microswimmers (see Section 2.1) and so the nematic interaction range can be related to physical quantities of the more realistic microswimmers behind. For microswimmers which are modeled as a rigid dumbbell the active volume is given by $V_{\text {active }}=l \bar{a}\left(v l^{2} / D\right)$ with $l$ being the length of the dumbbell, $\bar{a}=\left(a_{L}+a_{S}\right) / 2$ being the mean size of the two spheres, and $D$ being the diffusion constant (Baskaran and Marchetti, 2009). The idea behind the dumbbell model is a stroke-averaged microswimmer so that the two spheres represent the cell body and the volume where the flagella perform their stroke motion. It is important to stress here that the velocity $v$ is not the selfpropulsion speed of the particle but is the convective velocity which is proportional to the strength of the force dipole divided by the friction of the dumbbell in the ambient fluid. We can conclude for our model that the nematic interaction range depends not only on the length of the swimmer, but also on the size of the cell body, the stroke radius, and the strength of the corresponding force dipole. The diffusion also plays a role as well as friction due to the ambient fluid. This interpretation of the nematic interaction range means that different organisms will be subject to clustering in different turbulent flows, i.e. turbulent flows with different integral length scales of the vorticity. This is a possible route to solve the "paradox of the plankton" (Hutchinson, 1961) because it favors the clustering of different kinds of organisms in different regions. This spatial separation of different species hence increases the possibility of e.g. sexual mating for a given species because ideally only individuals of the same species cluster in one region in space. Different species can thus coexist in the same water body because they are effectively separated in space. Coming back to the illustration of the situation of phytoplankton in the ocean (see Fig. 1.4 in the introduction of this thesis), the situation is the following (see Fig. 6.14 for an illustration): A turbulent flow field can be induced in the water e.g. by wind (blue arrow). If the integral length scale of the vorticity does differ in different parts of the water (depicted by differently sized spirals), the different species can cluster in different regions according to their size. This separates the different species in space so that they can coexist.

Even for species with a similar active volume the clustering itself is possibly enough to lead to species diversity. Such a plankton population with different species of similar size leads to species-diverse clusters in the first step. If the clusters are stable compared to the typical time scale in the reproduction cycle, in each cluster a single species might survive due to competitive exclusion. However, because the small-scale conditions vary between clusters different species might survive in different clusters so that we globally find coexistence of competing species. This interpretation does not even need the formation of different turbulent field with different integral length scales of the vorticity. 


\section{Conclusion and Outlook}

\subsection{Conclusion}

This thesis posed the following research questions: What kind of patterns can emerge in three-dimensional suspensions of self-propelled, aligning, low-Reynolds number swimmers mimicking entities like bacteria and how do these patterns fit in the steadystate phase space? Are simple physical interactions enough to trigger the formation of complex patterns like propagating waves? How can the symmetry of the system be broken? What is the influence of an external flow field on the properties of large groups of self-propelled particles? Or asked differently, how does turbulence influence large groups of motile plankton in their natural habitats - lakes and oceans?

To address these questions we perform large-scale molecular dynamics simulations to solve the corresponding equations of motion. The individual particles align nematically and are subject to rotational diffusion. They are self-propelled with a constant speed. An external flow field is modeled via the method of kinematic simulations to acquire reasonable computation times and a turbulence-like behavior. This external flow field advects and rotates the particles according to the local velocity and vorticity. We also include the possibility of a finite size of the particles resulting in steric interactions among individuals.

The system under investigation is intrinsically out of equilibrium because each selfpropelled particle moves at a constant speed while it still interacts with its neighbors. This is only possible if energy is constantly converted into motion from some internal reservoir. Even though our model does not include this process explicitly, it is the reason why we refer to our system as a nonequilibrium system. Almost all of 
our simulations start from the most disordered state one might think of: The particles' positions are randomized in the simulation domain and the orientations are distributed randomly as well. This is what we call a homogeneous and isotropic state. In the temporal evolution the system undergoes a transient evolution until it reaches a steady state where the configuration of both positions and orientations is temporally stable.

We observe different kinds of symmetry breaking depending on the system parameters like number density $\rho$ and rotational Péclet number $\mathcal{P}$. The latter serves to compare the strength of nematic alignment and with the rotational noise. With increasing density and Péclet number the rotational symmetry of the systems is broken and global nematic alignment occurs. At low densities, this transition is accompanied by a coexistence state of nematically ordered and isotropic domains in the system. Such a coexistence leads to an inhomogeneous density distribution because particles accumulate in the nematic domains and as a result the spatial symmetry is broken. Deep in the nematic phase a spontaneous chiral symmetry breaking occurs through the formation of helices of the local nematic directors. It can be shown that this pattern is indeed a stable configuration of the underlying equations of motion and forms due to the delicate interplay between rotational fluctuations and alignment. In a one-dimensional system of non-moving rotors under a rapid quench (similar to the $X Y$-model) such a chiral symmetry breaking can also occur in terms of trapped spin waves. When the chiral symmetry is broken the system of SPPs also shows oscillations in the global polarization and hence a breaking of the nematic symmetry. This is coupled to a density wave traveling along the helical axis so that the particles are inhomogeneously distributed. A symmetry breaking of nematic symmetry is also observed at small densities close to the isotropic-nematic transition where the system exhibits propagating waves which are locally polarly ordered. At the same time the homogeneity of the system is broken because the waves appear in a soliton-like fashion with ordered, highly dense domains interrupted by a isotropic, dilute gas. Such waves were observed in two dimensional systems before and could be shown to be the outcome of an instability. We find that similar waves also occur in three dimensions and with nematic (instead of polar) alignment. In systems with strong nematic interaction and at low global densities the system of self-propelled particles even exhibits a breaking of the global nematic symmetry with the onset of global polar order. The local interaction between particles leads to the coupling of strong alignment and large local density because polarly aligned particles stick together if the Péclet number is large enough and the alignment mechanism is fast compared to the flight time of a particle through the interaction range. A nematic domain is formed by two such counter-propagating subgroups of self-propelled particles.

To address the question of the influence of a turbulent flow field onto motile plankton, we model self-propelled, aligning particles (as before) in an external turbulent flow field. Global alignment occurs if the stochastic noise and the turbulent field 
are weak compared to the nematic interaction strength. Our main finding is that turbulence can enhance clustering in terms of the formation of small-scale patches of (self-propelled) particles. The conditions for cluster formation are: (i) the system is in the nematic state, and (ii) the integral length scale of the vorticity is comparable to (at least a quarter of) the nematic interaction range. The strongest clustering occurs if the relevant length and time scales match: twice the integral length scale of the turbulent vorticity must equal the nematic interaction range, and the Kolmogorov time scale must agree with the time scale of the nematic alignment.

Moreover, the clustering is a possible ingredient to the resolution of the plankton paradox in two different ways: (i) due to necessity of matching length scales differently sized species might cluster in different turbulent flows. This leads to an effective spatial separation of species which leads to coexistence. (ii) If the clusters are longlived compared to the reproduction cycle of plankton species, similarly sized plankton species might form mixed species clusters. In each cluster one species survives by competitive exclusion but globally this might lead to coexistence of different species.

One large criticism of our model concerns the usage of periodic boundary conditions. It is probably the most common choice for MD simulations (also used in the Vicsek model) in order to avoid boundary effects. However, in models like ours particles leave the simulation domain very often due to their persistent motion and the PBC help in the formation of structure due to percolation across the boundaries. Increasing the system size can reduce this problem but the general criticism against PBC still holds. Moreover, the helical pattern can only be found with PBCs because its stability crucially depends on the symmetry that each infinitesimally thin, nematically ordered layer has two such neighboring layers. The angle between each pair of neighboring layers is constant so that the contributions on the torque of a given particle cancel out. All in all, PBC seems artificial and unrealistic since there is no real system which obeys PBC and some structures (like the helix) are only possible under these conditions. Two different approaches concerning the boundary conditions seem to be possible to overcome these problems: Firstly, one could model the system with open boundaries which could for example represent the situation of plankton in the open ocean. A second approach would be to add walls to the system which then mimics experimental setups of closed cells. Open boundaries work rather well for system where the individual components have a tendency to stay in the vicinity of each other. The nematic alignment mechanism instead would lead to at least two subgroups, each being highly polarized, traveling in opposite directions. The nematic symmetry of the interaction would then be less important because parallel alignment events would dominate. This would entirely shift the focus of interest which is why we did not pursue this idea further. So let's turn to the second approach: the reflective boundaries. Our system follows two equations of motion: one for the position and one for the orientation. The first step is to implement the walls by an additional force term acting on the positions. However, an alteration of the equation of motion 
of the orientations is also necessary because otherwise the particles would get stuck and constantly bump into the wall due to their persistent speed. We find that alignment at the wall leads to wall accumulation which is an unwanted boundary effect. Another idea is a discrete flip of the orientation if the particle encounters the wall. Even though this is a discrete operation in a time-continuous model we do not find strong boundary effects but instead a chiral (but not helical) pattern occurs (in a sandwich geometry). This result suggests that such reflective boundaries are worth studying in more detail. However, adding a turbulent field complicates the situation because the fluid flow also has to obey boundary conditions.

\subsection{Outlook}

Research is never limited to only providing answers but always leads to new questions as well. This section sketches possible future routes one might take starting from the work in this thesis.

A systematic study of the occurrence of the chiral pattern as a function of the length of the simulation domain would be interesting in analogy to the one-dimensional rotor model. In the latter long-range order is destroyed by the chiral patterns in the infinite system. The size-dependence of the occurrence of helices could hence provide insight into the question whether the system possesses true long-range order. A study of the nature of the phase transition would complete the picture of this nonequilibrium system. Moreover, the patterns occurring in our system are possibly linked to different instabilities in the underlying hydrodynamic theory. A linear stability analysis could provide insight similarly to the approach by Baskaran and Marchetti (2008b, 2012) but in three instead of two dimensions.

The results of the self-propelled particles in turbulence were qualitatively reproduced by a direct numerical simulation instead of the kinematic simulation technique. Still the questions arises how exactly features of the more realistic turbulent field might alter the results. These features include for instance intermittency as well as more realistic energy spectra. This question could be addressed by implementing the movement of the self-propelled particles into the DNS code to avoid storing large amount of data as it is done up to now. Investigating larger Reynolds numbers would be enlightening as for the general behavior of our model.

Another possible route would be to introduce two (or more) differently sized species into the model moving together in the same turbulent field. If our claim is correct that the formation of small-scale patches highly depends on the length scales of turbulent vorticity and nematic interaction, the accordingly sized species should cluster while a second, much smaller one should not.

We motivate our study of self-propelled particles in turbulence by motile phytoplankton which (among others) lives in the pycnocline. This is the layer in the water 
column which possesses the largest density gradient. Adding buoyancy to our particles would make the model more realistic by including the interplay between density gradient and particle density, and would allow the analysis of large-scale effects such as convection, which we have neglected. 



\section{A Appendix}

\section{A.1 Analytical expression for $C_{\text {random }}$ in the calculation of $Q$}

One drawback of the given definition of the patch concentration enhancement factor $Q$ (Eq. 3.77) is the inclusion of $C_{\text {random }}(f)$ which is calculated on the basis of a random configuration of particles. The significance of $Q$ thus depends crucially on the "randomness" of this random configuration of particles and is a function of the pseudo-random number generator as well as the placing algorithm. The latter is especially important in the case of extended particles to avoid overlaps between particles and then again even more important for denser packing than for less dense ones. It is hence tempting to deduce an analytical expression for $C_{\text {random }}(f)$. Typical distributions of the Voronoi volumes of randomly placed particles show an asymmetric bell-shaped curve (see Fig. A.1a). The curve for point-like particles is shifted towards smaller volumes as compared to extended particles and show a slightly broader distribution. The reason for this difference is the natural lower limit for a Voronoi volume of an extended particle which could be reached in random close packing. The derivation of such distributions is of particular interest in the field of granular materials. A gamma distribution function has been proposed (Kumar and Kumaran, 2005) and it is assumed to be close to Gaussian at high packing fractions (Oger et al., 1996). Due to this functional form of the distribution it is not as straightforward to calculate $C_{\text {random }}(f)$ analytically. A numerical calculation, however, results in smoothly decaying functions (see Fig. A.1b) which can be fitted by an exponential function multiplied with a power-law. Both curves for point-like and extended particles be- 


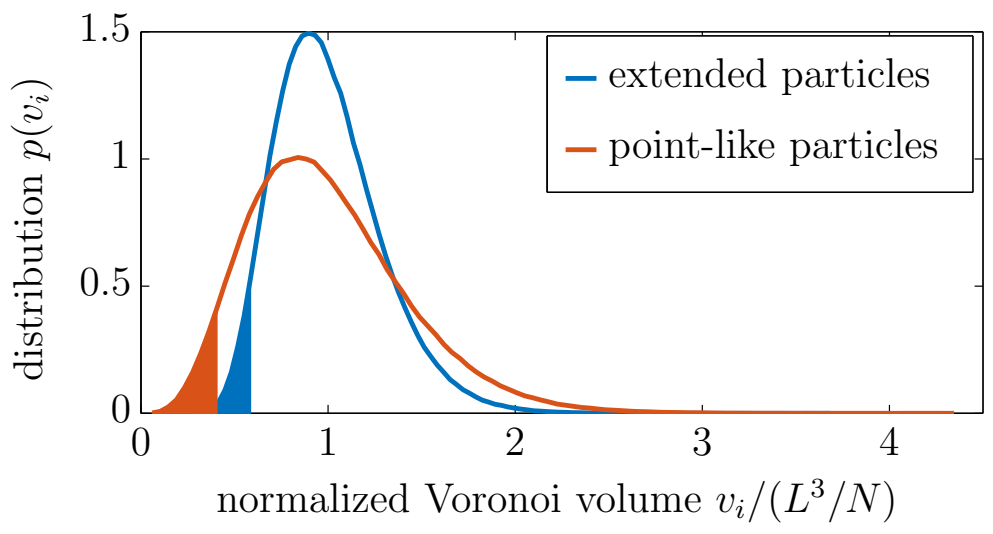

(a) Typical distributions of the Voronoi volumes of randomly placed particles. The shaded area indicates the $5 \%$ smallest Voronoi volumes used to calculate $C_{\text {random }}(f=0.05)$.

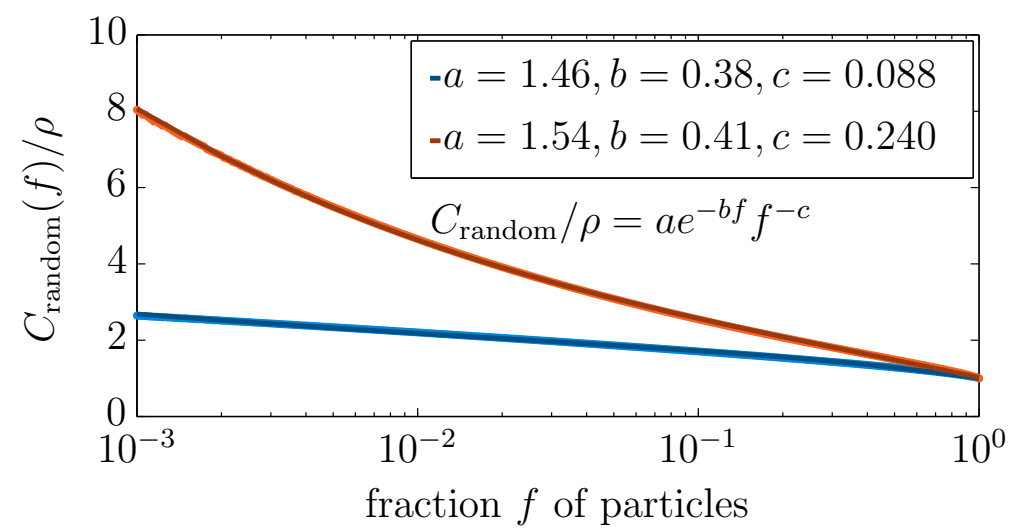

(b) $C_{\text {random }}$ as a function of the fraction $f$ of particles. The measurements are given as dots and the lines indicate fits of $C_{\text {random }}(f) / \rho=a \exp (-b f) f^{-c}$.

Figure A.1: Analysis of $C_{\text {random }}(f)$ for point-like particles (red) as well as extended particles (blue). The distributions are calculated numerically from $N=30^{3}$ particles in a box of side length $L=2 \pi$, packing fraction of extended particles $\phi=4 / 3 \pi(\sigma / 2)^{3} \rho=6.54 \%$. 
have very similar. We find that the prefactor $a$ of the fit functions as well as the fit parameter $b$ in the exponential agree rather well between the two curves. The exponent $c$ of the power-law, however, show a large difference between point-like and extended particles (roughly a factor of three). Since this functional form does not have a theoretical foundation (yet), we stick to calculating $C_{\text {random }}(f)$ from a random distribution of particles and leave the analytical derivation for future work.

\section{A.2 Isotropic-nematic transition: Variation of time step}

The simulations of point particles without turbulent field (see Chapter 4) are all carried out with a time step of $\Delta t=0.1$. The question remains whether this time step is small enough to resolve the dynamics of the system. To this end, we analyze simulations with varying $\Delta t$. In Fig. A.2, we observe that the isotropic-nematic transition is well-captured by all simulations and has the same shape for all time steps. This justifies the choice of $\Delta t=0.1$.

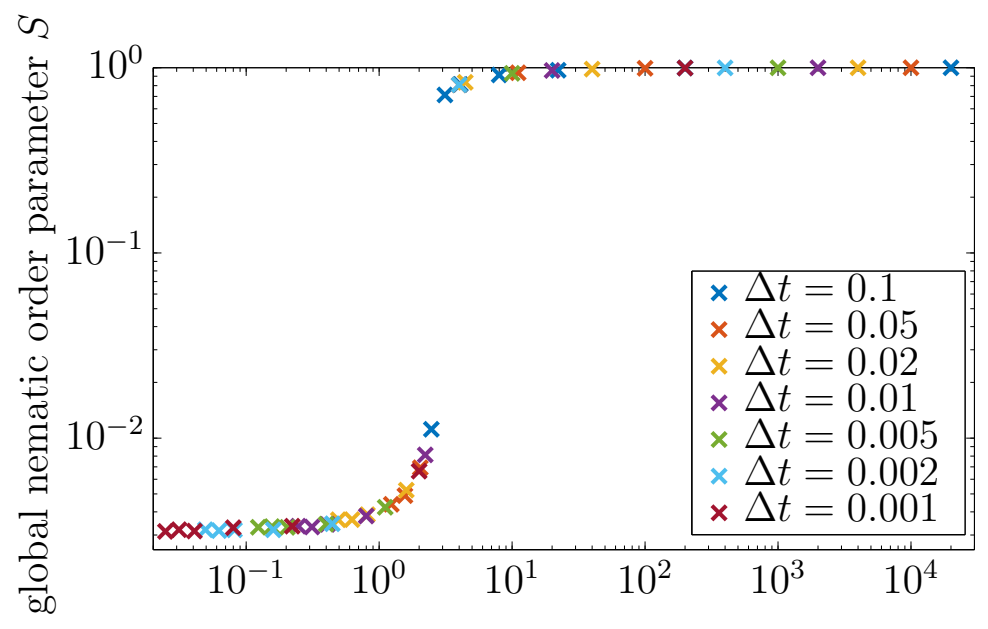

Péclet number $\mathcal{P}$

Figure A.2: Influence of the time step $\Delta t$ on the isotropic-nematic transition. All simulations are carried out with $N=64000$ particles, at a number density of $\rho=$ 1.125 , and with a nematic relaxation constant $\gamma=0.1$. Different colors correspond to different time steps as indicated. 


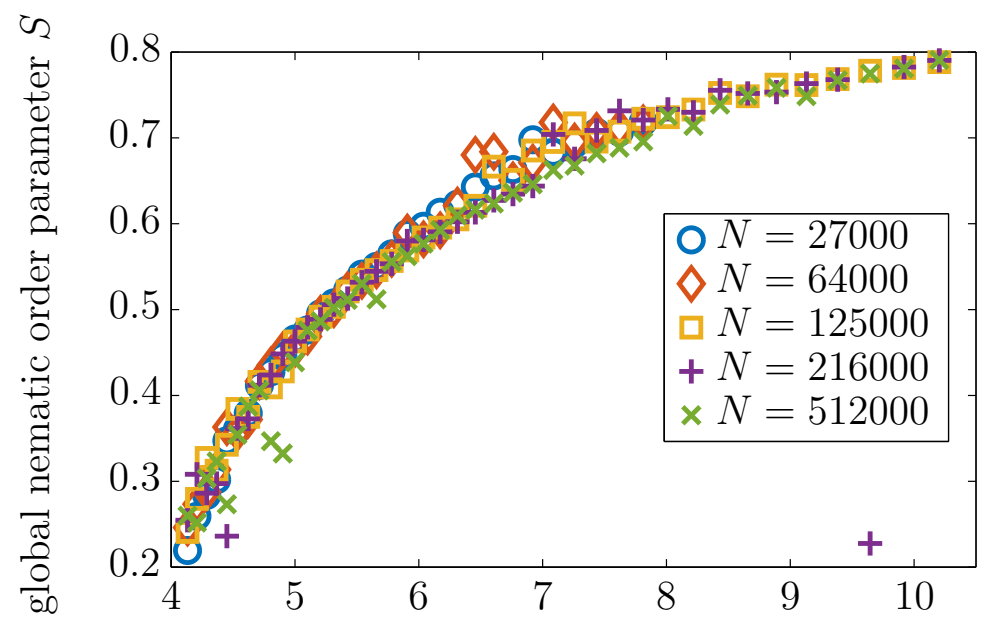

Péclet number $\mathcal{P}$

(a) Global nematic order parameter as a function of Péclet number at the isotropic-nematic transition. The value on the bottom right $(N=216000, P \approx 9.6, S=0.23)$ is much smaller than the neighboring values because it corresponds to a chiral simulation.

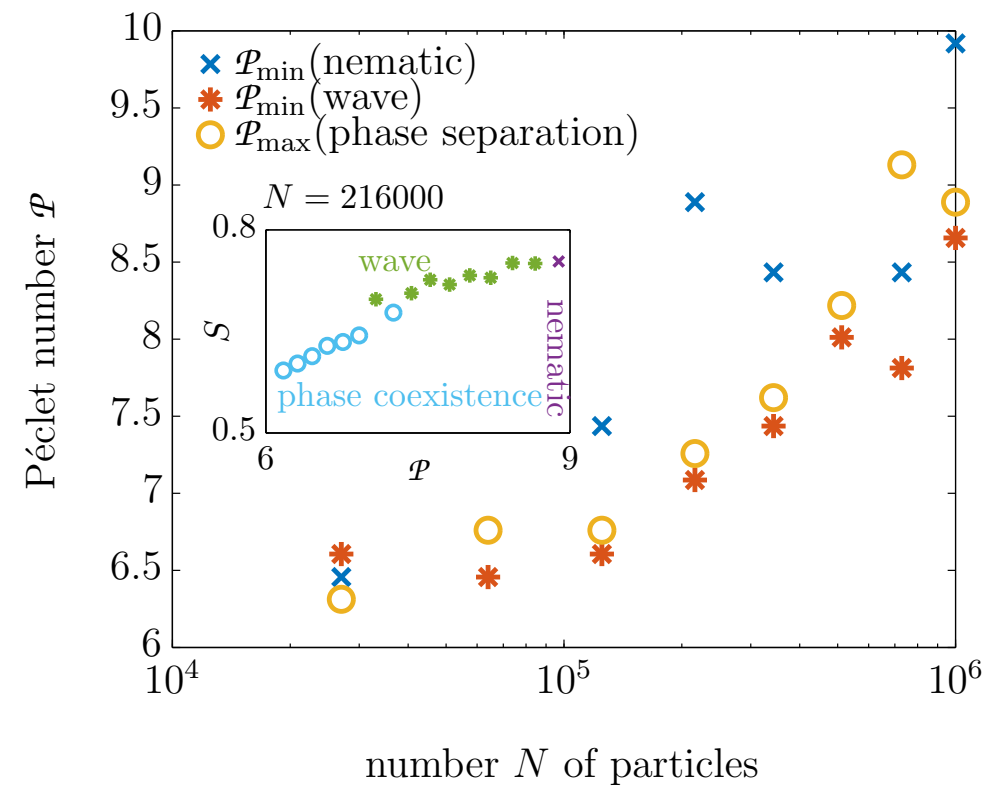

(b) The main panel shows three different definitions of the critical Péclet number as a function of particle number. The inset shows the isotropic-nematic transition for a system with $N=216000$ particles; different phases are marked differently to clarify the definitions of the critical Péclet number.

Figure A.3: Analysis of finite-size effects on the isotropic-nematic transition $(\rho=0.5$, $\gamma=1$ ) for different numbers of particles as indicated. 


\section{A.3 Isotropic-nematic transition: Finite size effects}

The curves of the global nematic order parameter as a function of the Péclet number for differently sized system with $27000 \leq N \leq 512000$ particles nicely collapse at the isotropic-nematic transition (Fig. A.3a). This suggests that no strong finite-size effects on the transition are to be expected.

However, the transition can be more exactly defined by a critical Péclet number. Due to the sequence of phases of the system of SPPs from isotropic, over phase coexistence and wave, to the nematic phase (with increasing Péclet number, see inset of Fig. A.3b), we use three different possible definitions of the critical Péclet number: The maximum Péclet number where phase coexistence is found, the minimum Péclet number where a polarized wave emerges, and the minimum Péclet number in the nematic state. The curves of these quantities as a function of system size (given by the number of particles, see Fig. A.3b) show a small positive trend. The critical Péclet number increases only from $\mathcal{P}_{c} \geq 6$ to $\mathcal{P} \approx 9$ while the number of particles varies over more than one and a half decades. This means that the nematic-isotropic transition is subject to a small finite-size effect.

\section{A.4 Code for three-dimensional monotonic logical grid}

This function finds pairs of neighboring particles to calculate interactions based on the fact that the particles in the structure molecule are ordered according to the monotonic logical grid as described in Section 3.2.2. For the sake of clarity of the code only interactions of point-like particles with periodic boundary conditions (PBCs) are considered.

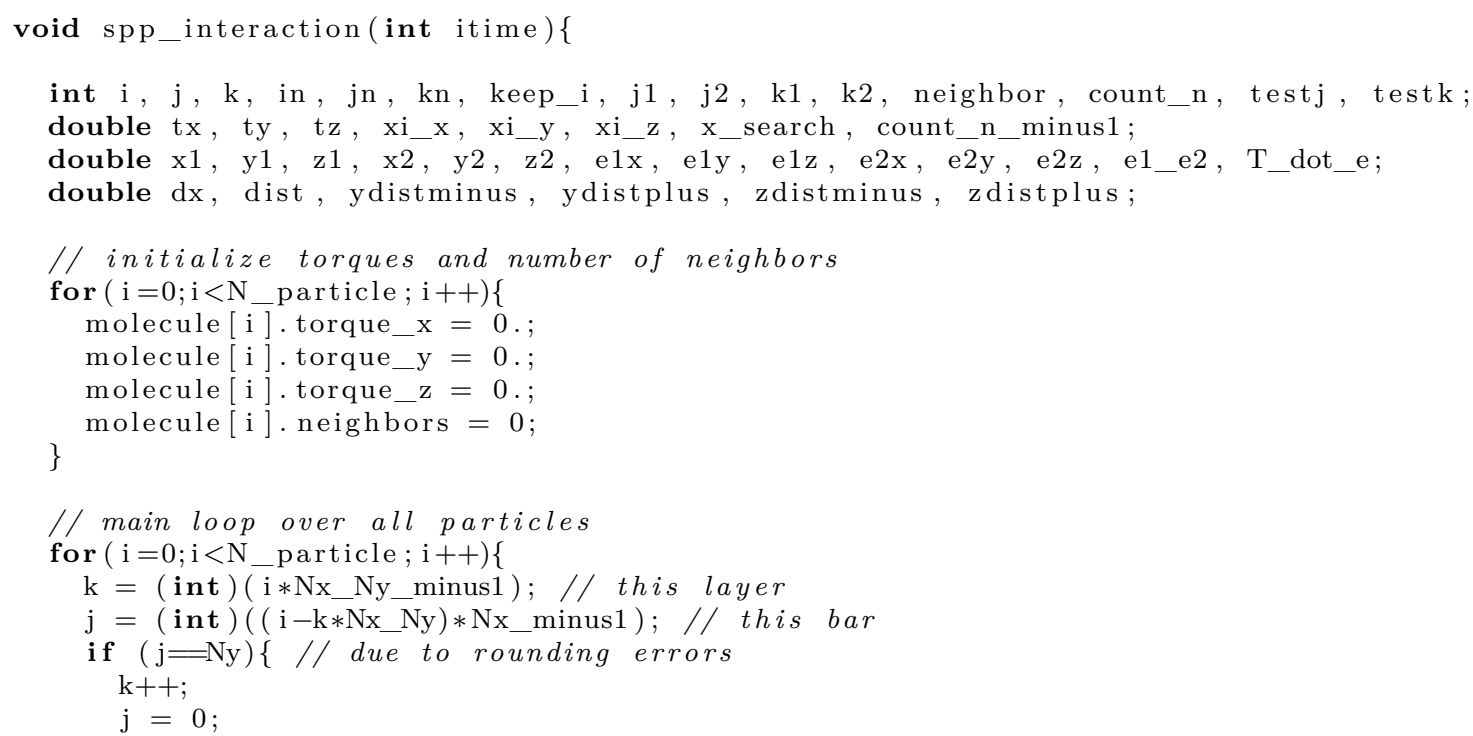




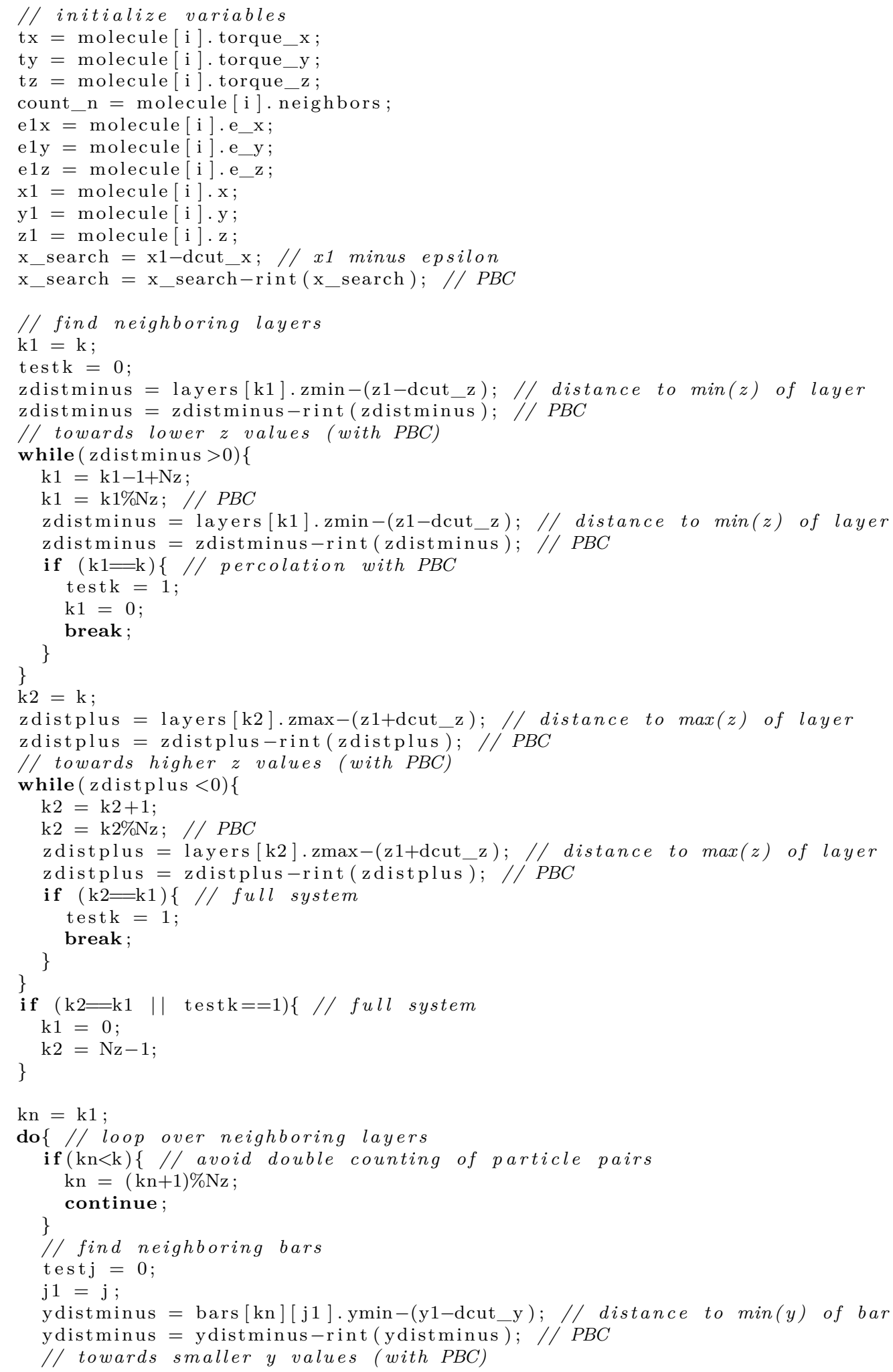




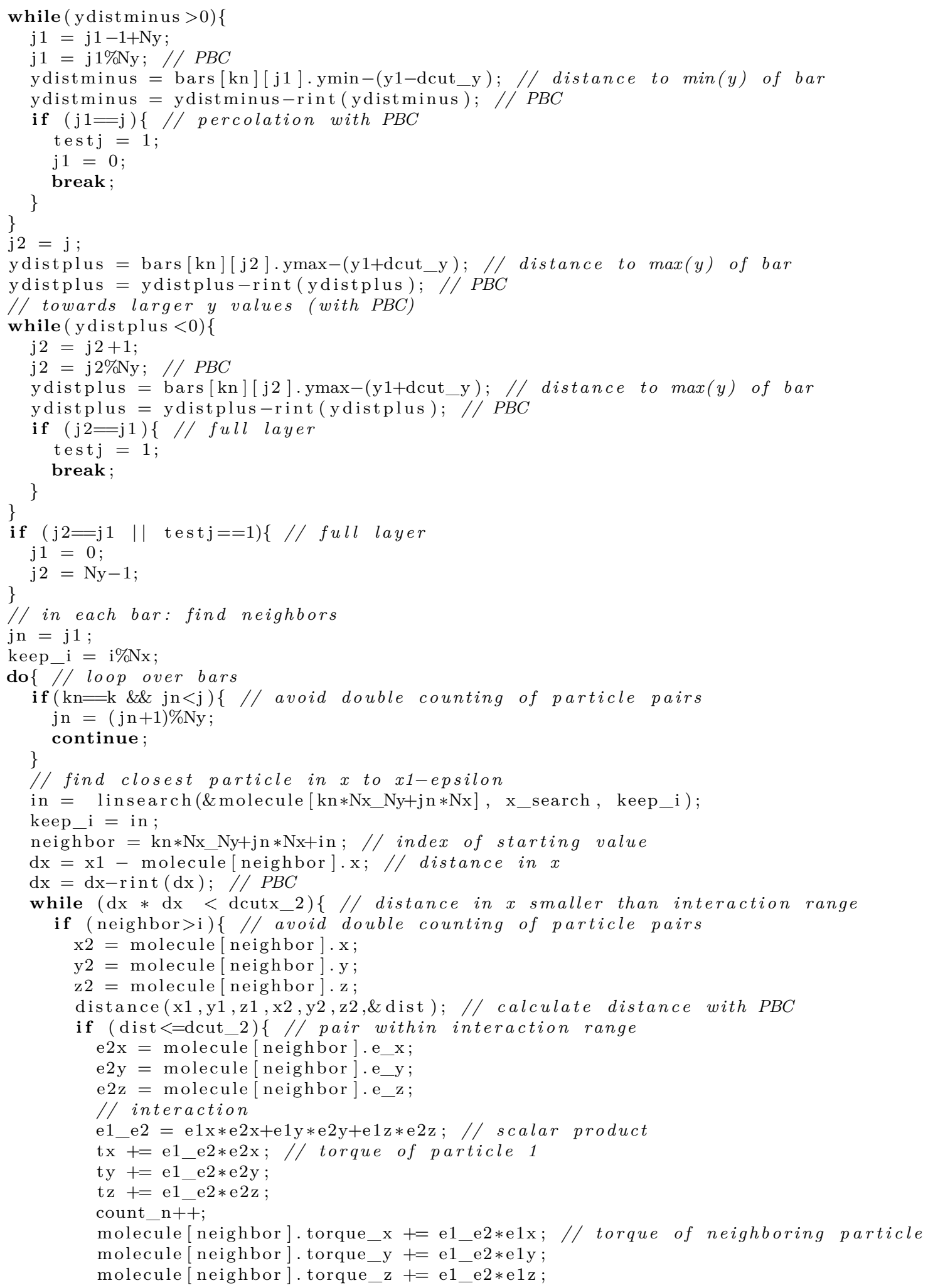




\section{A Appendix}

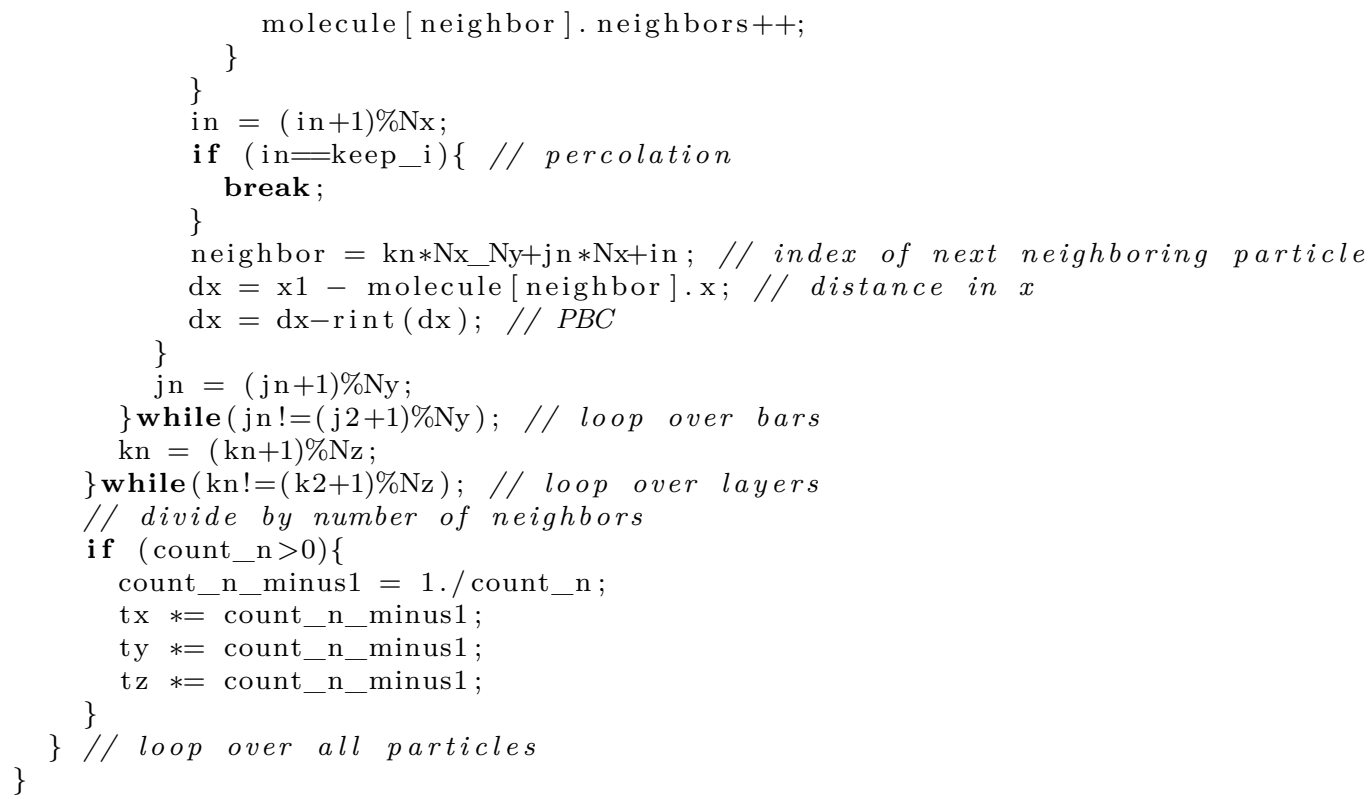




\section{Bibliography}

R. Aditi Simha and S. Ramaswamy. Hydrodynamic Fluctuations and Instabilities in Ordered Suspensions of Self-Propelled Particles. Physical Review Letters, 89(5): 058101, 2002.

R. Alexander and J. Imberger. Spatial distribution of motile phytoplankton in a stratified reservoir: the physical controls on patch formation. Journal of Plankton Research, 31(1):101-118, 2009.

M. P. Allen and D. J. Tildesley. Computer Simulation of Liquids. Oxford University Press, 1989.

I. Aranson. The Aquatic Dance of Bacteria. Physics, 6:61, 2013. doi: 10.1103/ Physics.6.61.

R. A. Armstrong and R. McGehee. Competitive Exclusion. The American Naturalist, 115(2):151-170, 1980.

W. D. Atkinson and B. Shorrocks. Competition on a Divided and Ephemeral Resource: A Simulation Model. Journal of Animal Ecology, 50(2):461-471, 1981.

A. Attanasi, A. Cavagna, L. Del Castello, I. Giardina, T. S. Grigera, A. Jelić, S. Melillo, L. Parisi, O. Pohl, E. Shen, and M. Viale. Information transfer and behavioural inertia in starling flocks. Nature Physics, 10(9):691-696, 2014.

M. Ballerini, N. Cabibbo, R. Candelier, A. Cavagna, E. Cisbani, I. Giardina, V. Lecomte, A. Orlandi, G. Parisi, A. Procaccini, M. Viale, and V. Zdravkovic. Interaction ruling animal collective behavior depends on topological rather than metric distance: Evidence from a field study. Proceedings of the National Academy of Sciences of the United States of America, 105(4):1232-1237, 2008.

L. Barberis and F. Peruani. Large-Scale Patterns in a Minimal Cognitive Flocking Model: Incidental Leaders, Nematic Patterns, and Aggregates. Physical Review Letters, 117(24):248001, 2016.

A. Baskaran and M. C. Marchetti. Enhanced Diffusion and Ordering of Self-Propelled Rods. Physical Review Letters, 101(26):268101, 2008a. 
A. Baskaran and M. C. Marchetti. Hydrodynamics of self-propelled hard rods. Physical Review E, 77(1):011920, 2008b.

A. Baskaran and M. C. Marchetti. Statistical mechanics and hydrodynamics of bacterial suspensions. Proceedings of the National Academy of Sciences of the United States of America, 106(37):15567-15572, 2009.

A. Baskaran and M. C. Marchetti. Self-regulation in self-propelled nematic fluids. The European Physical Journal E, 35(9):1-8, 2012.

E. Ben-Jacob, I. Cohen, O. Shochet, A. Tenenbaum, A. Czirók, and T. Vicsek. Cooperative Formation of Chiral Patterns during Growth of Bacterial Colonies. Physical Review Letters, 75(15):2899-2902, 1995.

E. Benincà, J. Huisman, R. Heerkloss, K. D. Jöhnk, P. Branco, E. H. Van Nes, M. Scheffer, and S. P. Ellner. Chaos in a long-term experiment with a plankton community. Nature, 451(7180):822-825, 2008.

H. C. Berg. Chemotaxis in Bacteria. Annual Review of Biophysics and Bioengineering, 4(1):119-136, 1975.

H. C. Berg, editor. E. coli in Motion. Biological and Medical Physics, Biomedical Engineering. Springer New York, 2004.

J. E. Berleman, J. Scott, T. Chumley, and J. R. Kirby. Predataxis behavior in Myxococcus xanthus. Proceedings of the National Academy of Sciences of the United States of America, 105(44):17127-17132, 2008. doi: 10.1073/pnas.0804387105.

E. Bertin, M. Droz, and G. Grégoire. Boltzmann and hydrodynamic description for self-propelled particles. Physical Review E, 74(2):022101, 2006.

E. Bertin, M. Droz, and G. Grégoire. Hydrodynamic equations for self-propelled particles: microscopic derivation and stability analysis. Journal of Physics A: Mathematical and Theoretical, 42(44):445001, 2009.

J. Boris. A vectorized "near neighbors" algorithm of order N using a monotonic logical grid. Journal of Computational Physics, 66(1):1-20, 1986.

A. Bracco, A. Provenzale, and I. Scheuring. Mesoscale vortices and the paradox of the plankton. Proceedings of the Royal Society of London Series B - Biological Sciences, 267(1454):1795-1800, 2000.

R. E. Breier, R. L. B. Selinger, G. Ciccotti, S. Herminghaus, and M. G. Mazza. Spontaneous chiral symmetry breaking in collective active motion. Physical Review E, 93(2):022410, 2016. doi: 10.1103/PhysRevE.93.022410. 
R. E. Breier, C. C. Lalescu, D. Waas, M. Wilczek, and M. G. Mazza. Emergence of phytoplankton patchiness at small scales in mild turbulence. under review, 2017.

A. Cavagna, A. Cimarelli, I. Giardina, G. Parisi, R. Santagati, F. Stefanini, and M. Viale. Scale-free correlations in starling flocks. Proceedings of the National Academy of Sciences of the United States of America, 107(26):11865-11870, 2010.

S. T. Chang, V. N. Paunov, D. N. Petsev, and O. D. Velev. Remotely powered selfpropelling particles and micropumps based on miniature diodes. Nature Materials, 6(3):235-240, 2007.

H. Chaté, F. Ginelli, and R. Montagne. Simple Model for Active Nematics: QuasiLong-Range Order and Giant Fluctuations. Physical Review Letters, 96(18):180602, 2006 .

H. Chaté, F. Ginelli, G. Grégoire, F. Peruani, and F. Raynaud. Modeling collective motion: variations on the Vicsek model. The European Physical Journal B, 64 (3-4):451-456, 2008a.

H. Chaté, F. Ginelli, G. Grégoire, and F. Raynaud. Collective motion of self-propelled particles interacting without cohesion. Physical Review E, 77(4):046113, 2008b.

O. Chepizhko, E. G. Altmann, and F. Peruani. Optimal Noise Maximizes Collective Motion in Heterogeneous Media. Physical Review Letters, 110(23):238101, 2013.

A. Czirók, M. Vicsek, and T. Vicsek. Collective motion of organisms in three dimensions. Physica A: Statistical Mechanics and its Applications, 264(1-2):299-304, 1999.

C. S. Davis, S. M. Gallager, and A. R. Solow. Microaggregations of Oceanic Plankton Observed by Towed Video Microscopy. Science, 257(5067):230, 1992.

P.-G. De Gennes and J. Prost. The Physics of Liquid Crystals, volume 23. Clarendon press, Oxford, 1993.

B. Descamps-Julien and A. Gonzalez. Stable Coexistence in a Fluctuating Environment: An Experimental Demonstration. Ecology, 86(10):2815-2824, 2005.

J. K. G. Dhont. An Introduction to Dynamics of Colloids. Elsevier, 1996.

W. M. Durham, E. Climent, M. Barry, F. De Lillo, G. Boffetta, M. Cencini, and R. Stocker. Turbulence drives microscale patches of motile phytoplankton. Nature Communications, 4:2148, 2013. 
W. Ebenhöh. Coexistence of an unlimited number of algal species in a model system. Theoretical Population Biology, 34(2):130-144, 1988.

B. Eggersdorfer and D.-P. Häder. Phototaxis, gravitaxis and vertical migrations in the marine dinoflagellate Prorocentrum micans. FEMS Microbiology Letters, 85 (4):319-326, 1991.

U. Erdmann and W. Ebeling. Collective motion of brownian particles with hydrodynamic interactions. Fluctuation and Noise Letters, 03(02):L145-L154, 2003.

U. Erdmann, W. Ebeling, and A. S. Mikhailov. Noise-induced transition from translational to rotational motion of swarms. Physical Review E, 71(5):051904, 2005.

B. M. Friedrich and F. Jülicher. Steering Chiral Swimmers along Noisy Helical Paths. Physical Review Letters, 103(6):068102, 2009.

U. Frisch. Turbulence - The legacy of A.N. Kolmogorov. Cambridge Univ Press, Cambridge, 1995.

J. C. H. Fung, J. C. R. Hunt, N. A. Malik, and R. J. Perkins. Kinematic simulation of homogeneous turbulence by unsteady random Fourier modes. Journal of Fluid Mechanics, 236:281-318, 1992.

A. Garcimartín, J. M. Pastor, L. M. Ferrer, J. J. Ramos, C. Martín-Gómez, and I. Zuriguel. Flow and clogging of a sheep herd passing through a bottleneck. Physical Review E, 91(2):022808, 2015. doi: 10.1103/PhysRevE.91.022808.

D. T. Gillespie. A theorem for physicists in the theory of random variables. American Journal of Physics, 51(6):520-533, 1983.

F. Ginelli and H. Chaté. Relevance of Metric-Free Interactions in Flocking Phenomena. Physical Review Letters, 105(16):168103, 2010.

F. Ginelli, F. Peruani, M. Bär, and H. Chaté. Large-Scale Collective Properties of Self-Propelled Rods. Physical Review Letters, 104(18):184502, 2010.

F. Ginelli, F. Peruani, M.-H. Pillot, H. Chaté, G. Theraulaz, and R. Bon. Intermittent collective dynamics emerge from conflicting imperatives in sheep herds. Proceedings of the National Academy of Sciences of the United States of America, 112(41): 12729-12734, 2015.

A. Gragnani, M. Scheffer, and S. Rinaldi. Top-Down Control of Cyanobacteria: A Theoretical Analysis. The American Naturalist, 153(1):59-72, 1999. 
G. Grégoire and H. Chaté. Onset of Collective and Cohesive Motion. Physical Review Letters, 92(2):025702, 2004.

G. Grosjean, M. Hubert, G. Lagubeau, and N. Vandewalle. Realization of the NajafiGolestanian microswimmer. Physical Review E, 94(2):021101, 2016.

D. Grossman, I. S. Aranson, and E. B. Jacob. Emergence of agent swarm migration and vortex formation through inelastic collisions. New Journal of Physics, 10(2): 023036, 2008.

R. Großmann, P. Romanczuk, M. Bär, and L. Schimansky-Geier. Vortex Arrays and Mesoscale Turbulence of Self-Propelled Particles. Physical Review Letters, 113(25): 258104, 2014.

R. Großmann, F. Peruani, and M. Bär. Mesoscale pattern formation of self-propelled rods with velocity reversal. Physical Review E, 94(5):050602, 2016.

J. S. Guasto, R. Rusconi, and R. Stocker. Fluid Mechanics of Planktonic Microorganisms. Annual Review of Fluid Mechanics, 44(1):373-400, 2012.

K. Gustavsson, F. Berglund, P. Jonsson, and B. Mehlig. Preferential Sampling and Small-Scale Clustering of Gyrotactic Microswimmers in Turbulence. Physical Review Letters, 116(10):108104, 2016.

E. Haeckel. Kunstformen der Natur. Verlag des Bibliographischen Instituts, Leipzig, 1899.

B. t. Hagen, F. Kümmel, R. Wittkowski, D. Takagi, H. Löwen, and C. Bechinger. Gravitaxis of asymmetric self-propelled colloidal particles. Nature Communications, 5:4829, 2014.

G. J. Hancock. The Self-Propulsion of Microscopic Organisms through Liquids. Proceedings of the Royal Society of London A: Mathematical, Physical and Engineering Sciences, 217(1128):96-121, 1953.

N. A. Hill and T. J. Pedley. Bioconvection. Fluid Dynamics Research, 37(1-2):1, 2005.

J. Huisman, A. M. Johansson, E. O. Folmer, and F. J. Weissing. Towards a solution of the plankton paradox: the importance of physiology and life history. Ecology Letters, 4(5):408-411, 2001.

G. E. Hutchinson. The Paradox of the Plankton. The American Naturalist, 95(882): 137-145, 1961. 
T. Ihle. Kinetic theory of flocking: Derivation of hydrodynamic equations. Physical Review E, 83:030901, 2011.

T. Ihle. Invasion-wave-induced first-order phase transition in systems of active particles. Physical Review E, 88(4):040303, 2013.

B. Ilkanaiv, D. B. Kearns, G. Ariel, and A. Be'er. Effect of Cell Aspect Ratio on Swarming Bacteria. Physical Review Letters, 118(15):158002, 2017.

J. M. Ilnytskyi and M. R. Wilson. A domain decomposition molecular dynamics program for the simulation of flexible molecules of spherically-symmetrical and nonspherical sites. II. Extension to NVT and NPT ensembles. Computer Physics Communications, 148(1):43-58, 2002.

T. Ishihara, Y. Kaneda, M. Yokokawa, K. Itakura, and A. Uno. Small-scale statistics in high-resolution direct numerical simulation of turbulence: Reynolds number dependence of one-point velocity gradient statistics. Journal of Fluid Mechanics, 592:335-366, 2007.

T. Ishikawa, M. P. Simmonds, and T. J. Pedley. Hydrodynamic interaction of two swimming model micro-organisms. Journal of Fluid Mechanics, 568(1):119-160, 2006 .

H. Jiang, H. Ding, M. Pu, and Z. Hou. Emergence of collective dynamical chirality for achiral active particles. Soft Matter, 2016.

Y. Katz, K. Tunstrøm, C. C. Ioannou, C. Huepe, and I. D. Couzin. Inferring the structure and dynamics of interactions in schooling fish. Proceedings of the National Academy of Sciences of the United States of America, 108(46):18720-18725, 2011.

J. O. Kessler. Hydrodynamic focusing of motile algal cells. Nature, 313(5999):218 $220,1985$.

A. N. Kolmogorov. The local Structure of turbulence in incompressible viscous fluid for very large Reynolds numbers [In Russian]. In Doklady Akademii Nauk SSSR, volume 30, pages 299-303, 1941.

R. H. Kraichnan. Diffusion by a Random Velocity Field. Physics of Fluids, 13(1): 22-31, 1970.

V. S. Kumar and V. Kumaran. Voronoi cell volume distribution and configurational entropy of hard-spheres. The Journal of Chemical Physics, 123(11):114501, 2005.

P. K. Kundu and I. M. Cohen. Fluid Mechanics. Elsevier, 4 edition, 2008. 
C. Lalli and T. Parsons. Biological Oceanography: An Introduction - 2nd ed. Elsevier Butterworth-Heinemann, Oxford, 1993.

P. Langevin. Sur la théorie du mouvement brownien. Comptes Rendus de l'Académie des Sciences (Paris), 146(530-533):530, 1908.

E. Lauga and T. R. Powers. The hydrodynamics of swimming microorganisms. Reports on Progress in Physics, 72(9):096601, 2009.

P. A. Lebwohl and G. Lasher. Nematic-Liquid-Crystal Order-A Monte Carlo Calculation. Physical Review A, 6(1):426-429, 1972.

J. E. Lennard-Jones. Cohesion. Proceedings of the Physical Society, 43(5):461, 1931.

S. A. Levin. Dispersion and Population Interactions. The American Naturalist, 108 (960):207-228, 1974.

H. Levine, E. Ben-Jacob, I. Cohen, and W.-J. Rappel. Swarming patterns in Microorganisms: Some new modeling results. In 2006 45th IEEE Conference on Decision and Control, pages 5073-5077, 2006.

D. Lewis and T. J. Pedley. Planktonic Contact Rates in Homogeneous Isotropic Turbulence: Theoretical Predictions and Kinematic Simulations. Journal of Theoretical Biology, 205(3):377-408, 2000.

J. Lighthill. Mathematical Biofluiddynamics. SIAM, 1975.

T. S. Lundgren. Linearly forced isotropic turbulence. Annual Research Briefs (Center for Turbulence Research, Stanford), pages 461-473, 2003.

E. Lushi, H. Wioland, and R. E. Goldstein. Fluid flows created by swimming bacteria drive self-organization in confined suspensions. Proceedings of the National Academy of Sciences of the United States of America, 111(27):9733-9738, 2014. doi: 10.1073/pnas.1405698111.

M. Maar, T. G. Nielsen, A. Stips, and A. Visser. Microscale distribution of zooplankton in relation to turbulent diffusion. Limnology and Oceanography, 48(3): 1312-1325, 2003.

N. A. Malik. Structural Diffusion in 2d and 3d Random Flows. In S. Gavrilakis, L. Machiels, and P. A. Monkewitz, editors, Advances in Turbulence VI, number 36 in Fluid Mechanics and its Applications, pages 619-620. Springer Netherlands, 1996. 
N. A. Malik and J. C. Vassilicos. A Lagrangian model for turbulent dispersion with turbulent-like flow structure: Comparison with direct numerical simulation for twoparticle statistics. Physics of Fluids, 11(6):1572-1580, 1999.

Z. Mansoori, M. Saffar-Avval, H. Basirat Tabrizi, and G. Ahmadi. Modeling of heat transfer in turbulent gas-solid flow. International Journal of Heat and Mass Transfer, 45(6):1173-1184, 2002.

M. C. Marchetti, J. F. Joanny, S. Ramaswamy, T. B. Liverpool, J. Prost, M. Rao, and R. A. Simha. Hydrodynamics of soft active matter. Reviews of Modern Physics, 85(3):1143-1189, 2013.

P. Mariani, B. R. MacKenzie, A. W. Visser, and V. Botte. Individual-based simulations of larval fish feeding in turbulent environments. Marine Ecology Progress Series, 347:155-169, 2007.

G. Maruyama. Continuous Markov processes and stochastic equations. Rendiconti del Circolo Matematico di Palermo, 4(1):48-90, 1955.

M. Matsumoto and T. Nishimura. Mersenne Twister: A 623-dimensionally Equidistributed Uniform Pseudo-random Number Generator. ACM Transactions on Modeling and Computer Simulation, 8(1):3-30, 1998.

B. C. Mazzag, I. B. Zhulin, and A. Mogilner. Model of Bacterial Band Formation in Aerotaxis. Biophysical Journal, 85(6):3558-3574, 2003.

W. L. McMillan. Simple Molecular Model for the Smectic $A$ Phase of Liquid Crystals. Physical Review A, 4(3):1238-1246, 1971.

U. Meierhenrich. Amino Acids and the Asymmetry of Life: Caught in the Act of Formation. Springer, 2008.

R. Memmer. Computer simulation of chiral liquid crystal phases VIII. Blue phases of the chiral Gay-Berne fluid. Liquid Crystals, 27(4):533-546, 2000.

A. M. Menzel. Collective motion of binary self-propelled particle mixtures. Physical Review E, 85(2):021912, 2012.

A. M. Menzel and T. Ohta. Soft deformable self-propelled particles. Europhysics Letters, 99(5):58001, 2012.

M. Meschede and O. Hallatschek. Local disalignment can promote coherent collective motion. New Journal of Physics, 15(4):045027, 2013. 
S. Mishra and S. Ramaswamy. Active Nematics Are Intrinsically Phase Separated. Physical Review Letters, 97(9), 2006.

A. S. Monin and A. M. Yaglom. Statistical Fluid Mechanics: Mechanics of Turbulence, volume 2. MIT Press, Cambridge, Mass, 1975.

M. Moussaïd, D. Helbing, and G. Theraulaz. How simple rules determine pedestrian behavior and crowd disasters. Proceedings of the National Academy of Sciences of the United States of America, 108(17):6884-6888, 2011.

K. H. Nagai, Y. Sumino, R. Montagne, I. S. Aranson, and H. Chaté. Collective Motion of Self-Propelled Particles with Memory. Physical Review Letters, 114(16): 168001, 2015.

M. Nagy, I. Daruka, and T. Vicsek. New aspects of the continuous phase transition in the scalar noise model (SNM) of collective motion. Physica A: Statistical Mechanics and its Applications, 373:445-454, 2007.

A. Najafi and R. Golestanian. Simple swimmer at low Reynolds number: Three linked spheres. Physical Review E, 69(6):062901, 2004.

S. Ngo, F. Ginelli, and H. Chaté. Competing ferromagnetic and nematic alignment in self-propelled polar particles. Physical Review E, 86(5):050101, 2012.

N. H. Nguyen, D. Klotsa, M. Engel, and S. C. Glotzer. Emergent Collective Phenomena in a Mixture of Hard Shapes through Active Rotation. Physical Review Letters, 112(7):075701, 2014.

A. Nicol, W. Rappel, H. Levine, and W. F. Loomis. Cell-sorting in aggregates of Dictyostelium discoideum. Journal of Cell Science, 112(22):3923-3929, 1999.

F. C. G. A. Nicolleau, M. Farhan, and A. F. Nowakowski. Effect of the energyspectrum law on clustering patterns for inertial particles subjected to gravity in kinematic simulation. Physical Review E, 94(4):043109, 2016.

L. Oger, A. Gervois, J. P. Troadec, and N. Rivier. Voronoi tessellation of packings of spheres: Topological correlation and statistics. Philosophical Magazine Part B, 74 (2):177-197, 1996.

T. Ohta and S. Yamanaka. Traveling bands in self-propelled soft particles. The European Physical Journal Special Topics, 223(7):1279-1291, 2014.

M. Omori and W. M. Hamner. Patchy distribution of zooplankton: Behavior, population assessment and sampling problems. Marine Biology, 72(2):193-200, 1982. 
D. J. G. Pearce, A. M. Miller, G. Rowlands, and M. S. Turner. Role of projection in the control of bird flocks. Proceedings of the National Academy of Sciences of the United States of America, 111(29):10422-10426, 2014.

F. Peruani, A. Deutsch, and M. Bär. A mean-field theory for self-propelled particles interacting by velocity alignment mechanisms. The European Physical Journal Special Topics, 157(1):111-122, 2008.

F. Peruani, J. Starruß, V. Jakovljevic, L. Søgaard-Andersen, A. Deutsch, and M. Bär. Collective Motion and Nonequilibrium Cluster Formation in Colonies of Gliding Bacteria. Physical Review Letters, 108(9):098102, 2012.

A. Peshkov, I. S. Aranson, E. Bertin, H. Chaté, and F. Ginelli. Nonlinear Field Equations for Aligning Self-Propelled Rods. Physical Review Letters, 109(26):268701, 2012 .

A. Peshkov, E. Bertin, F. Ginelli, and H. Chaté. Boltzmann-Ginzburg-Landau approach for continuous descriptions of generic Vicsek-like models. The European Physical Journal Special Topics, 223(7):1315-1344, 2014.

J. W. Pitchford and J. Brindley. Prey patchiness, predator survival and fish recruitment. Bulletin of Mathematical Biology, 63(3):527-546, 2001.

J. R. Platt. "Bioconvection Patterns" in Cultures of Free-Swimming Organisms. Science, 133(3466):1766-1767, 1961.

S. B. Pope. Turbulent flows. Cambridge Univ Press, New York, NY, USA, 2000.

G. Popkin. The physics of life. Nature, 529(7584):16-18, 2016. doi: 10.1038/529016a.

A. Posfai, T. Taillefumier, and N. S. Wingreen. Metabolic Trade-Offs Promote Diversity in a Model Ecosystem. Physical Review Letters, 118(2):028103, 2017.

E. M. Purcell. Life at low Reynolds number. American Journal of Physics, 45(1): $3-11,1977$.

S. Ramaswamy, R. A. Simha, and J. Toner. Active nematics on a substrate: Giant number fluctuations and long-time tails. Europhysics Letters, 62(2):196, 2003.

C. W. Reynolds. Flocks, herds and schools: A distributed behavioral model. In Proceedings of the 14th annual conference on Computer graphics and interactive techniques, SIGGRAPH '87, pages 25-34, New York, NY, USA, 1987. ACM.

L. F. Richardson. Atmospheric diffusion shown on a distance-neighbour graph. Proceedings of the Royal Society of London. Series A, Containing Papers of a Mathematical and Physical Character, 110(756):709-737, 1926. 
P. Richerson, R. Armstrong, and C. R. Goldman. Contemporaneous Disequilibrium, a New Hypothesis to Explain the "Paradox of the Plankton". Proceedings of the National Academy of Sciences of the United States of America, 67(4), 1970.

I. H. Riedel, K. Kruse, and J. Howard. A Self-Organized Vortex Array of Hydrodynamically Entrained Sperm Cells. Science, 309(5732):300-303, 2005.

H. Risken. The Fokker-Planck Equation: Methods of Solution and Applications. Springer, Berlin [u.a.], 1989.

P. Romanczuk, M. Bär, W. Ebeling, B. Lindner, and L. Schimansky-Geier. Active Brownian particles. The European Physical Journal Special Topics, 202(1):1-162, 2012.

M. Romensky, V. Lobaskin, and T. Ihle. Tricritical points in a Vicsek model of self-propelled particles with bounded confidence. Physical Review E, 90(6):063315, 2014 .

C. Rosales and C. Meneveau. Linear forcing in numerical simulations of isotropic turbulence: Physical space implementations and convergence properties. Physics of Fluids, 17(9):095106, 2005.

O. N. Ross and J. Sharples. Phytoplankton motility and the competition for nutrients in the thermocline. Marine Ecology Progress Series, 347:21-38, 2007.

S. Roy and J. Chattopadhyay. Towards a resolution of 'the paradox of the plankton': A brief overview of the proposed mechanisms. Ecological Complexity, 4(1-2):26-33, 2007.

S. Roy, S. Bhattacharya, P. Das, and J. Chattopadhyay. Interaction among Nontoxic Phytoplankton, Toxic Phytoplankton and Zooplankton: Inferences from Field Observations. Journal of Biological Physics, 33(1):1-17, 2007.

P. G. Saffman. The large-scale structure of homogeneous turbulence. Journal of Fluid Mechanics, 27(3):581-593, 1967.

D. Saintillan and M. J. Shelley. Instabilities and Pattern Formation in Active Particle Suspensions: Kinetic Theory and Continuum Simulations. Physical Review Letters, 100(17):178103, 2008.

V. Schaller, C. Weber, C. Semmrich, E. Frey, and A. R. Bausch. Polar patterns of driven filaments. Nature, 467(7311):73-77, 2010. 
V. Schaller, C. A. Weber, B. Hammerich, E. Frey, and A. R. Bausch. Frozen steady states in active systems. Proceedings of the National Academy of Sciences of the United States of America, 2011.

M. Scheffer, S. Rinaldi, J. Huisman, and F. J. Weissing. Why plankton communities have no equilibrium: solutions to the paradox. Hydrobiologia, 491(1-3):9-18, 2003.

L. Seuront, H. Yamazaki, and S. Souissi. Hydrodynamic Disturbance and Zooplankton Swimming Behavior. Zoological Studies, 43(2):376-387, 2004.

J. H. Steele. Spatial Heterogeneity and Population Stability. Nature, 248(5443): 83-83, 1974.

R. Stocker. Reverse and flick: Hybrid locomotion in bacteria. Proceedings of the National Academy of Sciences of the United States of America, 108(7):2635-2636, 2011.

J. Strefler, U. Erdmann, and L. Schimansky-Geier. Swarming in three dimensions. Physical Review E, 78(3):031927, 2008.

B. L. Taylor, I. B. Zhulin, and M. S. Johnson. Aerotaxis and Other Energy-Sensing Behavior in Bacteria. Annual Review of Microbiology, 53(1):103-128, 1999.

G. I. Taylor. Statistical Theory of Turbulence. Proceedings of the Royal Society of London A: Mathematical, Physical and Engineering Sciences, 151(873):421-444, 1935.

H. Tennekes and J. L. Lumley. A First Course in Turbulence. MIT Press, 1972.

D. J. Thomson and B. J. Devenish. Particle pair separation in kinematic simulations. Journal of Fluid Mechanics, 526:277-302, 2005.

S. Thutupalli, R. Seemann, and S. Herminghaus. Swarming behavior of simple model squirmers. New Journal of Physics, 13(7):073021, 2011.

P. Tiselius. Behavior of Acartia tonsa in patchy food environments. Limnology and Oceanography, 37(8):1640-1651, 1992.

J. Toner and Y. Tu. Long-Range Order in a Two-Dimensional Dynamical XY Model: How Birds Fly Together. Physical Review Letters, 75(23):4326-4329, 1995.

J. Toner and Y. Tu. Flocks, herds, and schools: A quantitative theory of flocking. Physical Review E, 58(4):4828-4858, 1998.

J. Toner, Y. Tu, and S. Ramaswamy. Hydrodynamics and phases of flocks. Annals of Physics, 318(1):170-244, 2005. 
S. Vedel, H. Nunns, A. Košmrlj, S. Semsey, and A. Trusina. Asymmetric Damage Segregation Constitutes an Emergent Population-Level Stress Response. Cell Systems, 3(2):187-198, 2016.

T. Vicsek and A. Zafeiris. Collective motion. Physics Reports, 517(3-4):71-140, 2012.

T. Vicsek, A. Czirók, E. Ben-Jacob, I. Cohen, and O. Shochet. Novel Type of Phase Transition in a System of Self-Driven Particles. Physical Review Letters, 75(6): 1226-1229, 1995. doi: 10.1103/PhysRevLett.75.1226.

A. W. Visser and G. A. Jackson. Characteristics of the chemical plume behind a sinking particle in a turbulent water column. Marine Ecology Progress Series, 283: 55-71, 2004.

A. W. Visser and T. Kiørboe. Plankton motility patterns and encounter rates. Oecologia, 148(3):538-546, 2006.

D. Waas. Turbulence Driven Clustering in Nematic Active Particles. Bachelor thesis, Università degli Studi di Milano, 2015.

L.-P. Wang and M. R. Maxey. Settling velocity and concentration distribution of heavy particles in homogeneous isotropic turbulence. Journal of Fluid Mechanics, 256:27-68, 1993.

J. D. Weeks, D. Chandler, and H. C. Andersen. Role of Repulsive Forces in Determining the Equilibrium Structure of Simple Liquids. The Journal of Chemical Physics, 54(12):5237-5247, 1971.

S. Weinketz. A two-dimensional monotonical logical grid algorithm. Computer Physics Communications, 74(2):228-232, 1993.

S. Weitz, A. Deutsch, and F. Peruani. Self-propelled rods exhibit a phase-separated state characterized by the presence of active stresses and the ejection of polar clusters. Physical Review E, 92(1):012322, 2015.

H. H. Wensink, J. Dunkel, S. Heidenreich, K. Drescher, R. E. Goldstein, H. Löwen, and J. M. Yeomans. Meso-scale turbulence in living fluids. Proceedings of the National Academy of Sciences of the United States of America, 109(36):1430814313, 2012.

M. Wilczek. Statistical and Numerical Investigations of Fluid Turbulence. PhD thesis, Westfälische Wilhelms-Universität Münster, 2010. 
H. Wioland, F. G. Woodhouse, J. Dunkel, J. O. Kessler, and R. E. Goldstein. Confinement Stabilizes a Bacterial Suspension into a Spiral Vortex. Physical Review Letters, 110(26):268102, 2013.

Y. Wu, A. D. Kaiser, Y. Jiang, and M. S. Alber. Periodic reversal of direction allows Myxobacteria to swarm. Proceedings of the National Academy of Sciences of the United States of America, 106(4):1222-1227, 2009.

A. Wysocki, R. G. Winkler, and G. Gompper. Cooperative motion of active Brownian spheres in three-dimensional dense suspensions. Europhysics Letters, 105(4):48004, 2014.

L. Xie, T. Altindal, S. Chattopadhyay, and X.-L. Wu. Bacterial flagellum as a propeller and as a rudder for efficient chemotaxis. Proceedings of the National Academy of Sciences of the United States of America, 108(6):2246-2251, 2011.

S. Yamanaka and T. Ohta. Formation and collision of traveling bands in interacting deformable self-propelled particles. Physical Review E, 89(1):012918, 2014.

C. Zhan, G. Sardina, E. Lushi, and L. Brandt. Accumulation of motile elongated micro-organisms in turbulence. Journal of Fluid Mechanics, 739:22-36, 2014.

H. Zhang, Z. Vaksman, D. B. Litwin, P. Shi, H. B. Kaplan, and O. A. Igoshin. The Mechanistic Basis of Myxococcus xanthus Rippling Behavior and Its Physiological Role during Predation. PLoS Computational Biology, 8(9):e1002715, 2012a.

Y. Zhang, A. Ducret, J. Shaevitz, and T. Mignot. From individual cell motility to collective behaviors: insights from a prokaryote, Myxococcus xanthus. FEMS Microbiology Reviews, 36(1):149-164, 2012b. 


\section{Acknowledgements}

This work would have never been completed without the support of so many people. Foremost, I want to thank Marco Mazza for allowing me to do my PhD with him, for all the freedom and support he gave me in the past years. He never stopped believing in my skills, let me follow my own ideas, but sometimes (at the right points) gently forced me back on track. I thank Stephan Herminghaus for hiring me and giving me the opportunity to do this work and for many useful discussions. I also highly acknowledge all the support of both of them during pregnancy and maternity leave. I thank Marcus Müller for discussions during thesis committee meetings and for agreeing to referee this thesis. Thanks also to Fabio Marchesoni for being a referee. I had the pleasure of collaborating with external people for my publications: I thank Giovanni Ciccotti for numerous long skype-meetings and incredible patience to understand what my system was about. Robin Selinger inspired and impresssed me; special thanks go to her for the one-dimensional model, detailed explanation of the physics behind, and last but not least the haiku. I thank Michael Wilczek for turbulence lessons and for asking the right questions to improve the research. And finally, I want to thank Cristian "Chichi" Lalescu for finding ways to provide me with loads of data and answering all my turbulence-beginners questions.

Research does not work without infrastructure behind it, so special thanks go to Barbara Kutz, Monika Teuteberg, Olaf Trümper, Kristian Hantke, and Thomas Eggers.

I very much enjoyed to be part of the nonequilibrium soft matter group where we had endless discussions on science and everything else (including font sizes and line styles). I thank James for being something like a big brother, Qiong for female company, Jeff for table tennis, Jack for chiral discussions, Fabi for being the true theorist among us, Tine for funny office times, André and Chenyu for office company, Henning for fresh thoughts on the self-propelled particles, Devin for teaching me how to explain things clearly, Tanya for being my roommate, Gal for "pink wash", and everybody else (also from the yellow floor in Bunsenstraße) for enjoyable lunches.

I cannot express how grateful I am to my husband Ali for being by my side through everything that happened in the past years, all the ups and downs. Thank you for all the support and discussions and encouragement (and for reading this whole thesis)! And last but not least, I am thankful that Frieda came to us and teaches us everyday a million different things. I am looking forward to discover the world with you! 



\section{Curriculum Vitae}

\section{Current address}

Rebekka Elisabeth Breier, née Heyn

Wieterweg 6

37081 Göttingen

Germany

\section{Personal information}

Date of birth November 26, 1987

Place of birth Göttingen, Germany

\section{Previous education}

10/2010-09/2012 Studies of Physics at Universität Rostock specialization "Oceanography and Physics of the Atmosphere"

Degree Master of Science (M.Sc.)

Thesis $\quad$ Effects of shear on high-frequency internal motions in the Baltic Sea

10/2007-09/2010 Studies of Physics at Martin-Luther-Universität Halle-Wittenberg Degree Bachelor of Science (B.Sc.)

Thesis Elektronische Struktur von vergrabenen Defekten

\section{Publications}

R. E. Breier, R. L. B. Selinger, G. Ciccotti, S. Herminghaus, and M. G. Mazza. Spontaneous chiral symmetry breaking in collective active motion. Physical Review E, 93 (2):022410, 2016.

R. E. Breier, C. C. Lalescu, D. Waas, M. Wilczek, and M. G. Mazza. Emergence of phytoplankton patchiness at small scales in mild turbulence. (under review, 2017). 



\section{Erklärung}

1. Ich habe diese Arbeit selbständig und ohne unerlaubte Hilfe angefertigt und dabei nur die angegebenen Quellen benutzt.

2. Eine entsprechende Promotion wurde an keiner anderen Hochschule im In- oder Ausland beantragt; die eingereichte Dissertation oder Teile von ihr wurden nicht für ein anderes Promotionsvorhaben verwendet.

Göttingen, 2. Mai 2017

Rebekka E. Breier 